\title{
Electrohydrodynamic Patterning of Functional Materials
}

\section{Pola Goldberg Oppenheimer}

A Dissertation Submitted for the Degree of Doctor of Philosophy July 2011

Supervisor

Prof. Dr. Ullrich Steiner

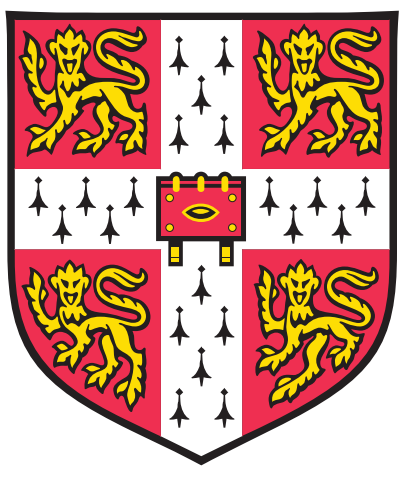

University of Cambridge

Cavendish Laboratories

Department of Physics

Churchill College 
To the three most precious women in my life and heart: My daughter-Roni My mother-Luda My late grandmother-Bathia 


\section{Declaration}

This dissertation is the result of my own work and includes nothing which is the outcome of work done in collaboration except where specifically indicated in the text. No part of this work has been submitted for a degree or any other qualification at this or any other University. This dissertation does not exceed the word limit of 60,000 words set by the Physics and Chemistry Degree Committee.

\section{Pola Goldberg Oppenheimer.}

July 2011 


\section{Abstract}

\section{Electrohydrodynamic Patterning of Functional Materials}

Pola Goldberg Oppenheimer

This thesis explores an alternative route to induce and control the structure formation process in thin films by the use of strong electric fields. The objective of this thesis is to investigate, establish and apply the use of the electrohydrodynamic (EHD) lithography as a versatile patterning tool on the sub-micrometre and nanometre length scales for functional materials in thin films. Thin films are ubiquitous, they are found in nature and used in almost every aspect of daily life. While film instabilities are often undesirable in nature and technology, they can be utilized to produce structures by precisely controlling the destabilization of the film. EHD lithography utilizes instabilities induced by means of an electric field to fabricate periodic structures. While still in the developing phase, EHD patterning is set to become a competitive candidate for low-cost lithographic technology for a number of applications. Herein, the applied potential of this lithographic process is explored by expanding its applicability to a broad range of materials and material combinations and by a simultaneous patterning of multilayer systems or functional polymers yielding hierarchical architectures with novel functionalities.

An intrinsic problem of the EHD patterning process is the rather long pattern generation times, which has to be overcome. A number of low-viscosity materials are exploited as high-speed resists to significantly reduce the patterning time of EHD to a few seconds. These results demonstrate the versatility of the EHD method and render it technologically appealing. Further work presents a route towards controlled reproducible alignment of carbon nanotubes (CNTs) during an EHD patterning process of nanocomposite. The degree of nanotube alignment is tuned by adjusting the EHD parameters and the degree of alignment is mirrored by the conductivity across the film. Patterned surfaces decorated by CNT brushes are also generated in this study. A method to create controlled self-organized hierarchical nanostructures using EHD instabilities is an additional topic of this thesis. Herein, pattern formation harnesses a sequential instability in multilayer thin films induced by an electric field to guide the layer material into design structures, allowing different materials to be patterned in a one-step procedure. This pattern formation enables the fabrication of multi-scale structured arrays as surface enhanced Raman scattering (SERS)-active platforms. Each of the formed structures is effectively tailored to provide high SERS enhancement.

Furthermore, crystalline and conductive polymers are patterned using the EHD approach and the underlying structure formation mechanisms are discussed. This extension towards functional material systems offers interesting prospects for potential applications. Finally, the EHD formation of hierarchical structures spanning several length scales is demonstrated. Confining conductive block copolymers in presence of an electric field into fabricated microstructures gives rise to an internal vertical alignment of the copolymers nanometric domains with an additional molecular orientation. Inside the superstructure films ordered arrays of nanocrystals of the constituting block are aligned in a smectic phase giving rise to birefringence. The alignment of the nanodomians improves the charge conduction toward the electrodes. These findings are very promising for use in optoelectronic devices. 


\section{Acknowledgements}

The work described in this thesis was carried out under the supervision of Prof. Ullrich Steiner head of the Thin Films and Interfaces Group in the Department of Physics, University of Cambridge. I would like to take this opportunity to express my appreciation and gratitude to all those who have contributed to this fascinating journey.

First and foremost, I would like to express my deepest gratitude to Prof. Ullrich Steiner for providing me with the exciting opportunity to carry out creative and stimulating research over the last few years. Prof. Steiner has provided wise guidance at all stages of the research, while at the same time allowing me freedom to explore my paths. He ensured invaluable practical as well as conceptual independence. Ulli has established an ideal explorative ambience with an international flavour within the group, along with the help and advice whenever they were needed. It was a pleasure to work alongside and learn from such an inspiring and extraordinary scientist, supervisor and most of all, person. I am sincerely grateful for his professional support as well as his open door and ear for all aspects of one's life.

The studies performed in the last years have been enriched by the deep insights of scientists from different backgrounds, with whom I was fortunate to interact throughout my course of study. This has been an incredible opportunity to learn and extend my knowledge within an international working atmosphere. I thank Dr. Sumeet Mahajan for the pleasant co-operation we had while working on the SERS project, for his patience and for the enlightening discussions about science and beyond. Dr. Peter Kohn provided valuable input into the world of polymer crystallinity and Dr. Dominik Eder extended my knowledge of carbon nanotubes and related topics. I am grateful to them both. I would like to thank Dr. Dinesh Kabra for a short but pleasant and stimulating collaboration and a glance into the experimental and applied world of optoelectronics.

Special thanks go to J.J. Rickard for his fruitful advice, for extending my knowledge on the wonders of the electron microscopy, for patience and for many hours spent while assisting me with microtoming challenging samples (and they been many). He has also been a great friend to me. I also wish to thank Dr. Richard Langford for assistance with diffraction pattern analysis.

Special words of appreciation to my colleagues in the research group: Dr. Sven Huttner, thank you for introducing and guiding me through the world of Physics and experimental techniques in the early days. The knowledge that you conveyed to me (sometimes with a cynical touch) will follow me in my future research career. Dr. Mathias Kolle, for sharing with me his excitement of science and of life both in- and outside the lab. It was a great pleasure working with you and having you as part of my Cambridge life. Special thanks to Stefan Guldin for fascinating and open discussions beyond science, for his openness and for being such a good friend all the way through. My gratitude also goes to Dr. Rosa Poetes, Dr. Mihaela Nedelcu, Dr. Ed Crossland and Dr. Katherine Thomas for their warm and generous support, to Dr. Natalya Yufa - for the goodwill and ambience and to Dr. Silvia Vignolini for the Italian touch, to Ellie Kim for being a good friend and listener, to Sarah Rong, Li Li, and Yuang Zhou for providing the authentic atmosphere and the delicious, but peculiar sweets. I also thank Alex Finnemore, Pedro Cunha, Stefano Salvatore 
and Gen Kamita for scientific insights, sincere support and a helpful hand. And last, but not least from the group, the Scherer brothers: Maik and Kai. I am particularly grateful to Maik for teaching me the world of LaTex, for his patient advice and in particular, for an open ear and friendship. It has been a pleasure being part of such a team, having endless scientific debates and also sharing time outside of the lab and office.

I would also wish to thank the colleagues and friends from the group of Nanophotonics. I thank Dr. Gabriel Christmann for his assistance through the Latex mazes, his kindness and friendship, Dr. Chris Finlyason, for productive collaboration and enjoyable discussions at social occasions, Peter Farah, Niraj Lal, Dr. Bruno Flavio Soares and Andreas Kontogeorgos all for their openness and amity. I am indebted to Angela Campbell, for her open ear, for her wise and warm advice and support, and for her invaluable friendship.

I wish to acknowledge the financial support I received from Kodak European Research, Cambridge.

For the introduction and guiding through the wonders of CorelDRAW and for the open and kind friendship I would like to thank Dr. Artem Bakulin.

Moving from one country to another while giving birth in a foreign country were not easy transitions for me and many compromises had to be made. Therefore, finally I wish to thank some special people who accompanied me all along with warm, loving support and encouragement in my fascinating, yet challenging journey: my family. Special words of gratitude to my mother, Luda for all the love and support all the way through. I thank my husband, Gal, for the support and in particular for a help with raising our wonderful child. And last, but not least, I would like to thank my daughter, Roni, for being my pride and joy and bringing a smile to my face even at the most challenging times. The work and the journey would not have been the same without you.

I am deeply grateful to you all. 


\section{List of Publications}

1. Structural Hierarchy of Functional Block Copolymer System Induced by Electrohydrodynamic Lithography. Goldberg Oppenheimer, P., Kabra, D., Huttner, S. and Steiner, U. In preparation, 2011.

2. Patterning of Crystalline Organic Materials via Electrohydrodynamic Lithography. Goldberg Oppenheimer, P., Kohn, P., Langford, R. and Steiner, U. In preparation, 2011.

3. Hierarchical Electrohydrodynamic (HEHD) Structures for Surface-Enhanced Raman Scattering. Goldberg Oppenheimer, P., Mahajan, S. and Steiner, U. In final revision before submission, 2011.

4. Electrically Conductive Polymeric Photonic Crystals. Imai, Y., Finlayson, C. E., Goldberg Oppenheimer, P., Zhao, Q., Spahn, P., Snoswell, D. R.E., Haines, A., Hellman, P. and Baumberg, J.J. Submitted to Advanced Functional Materials, 2011.

5. Carbon Nanotubes Alignment via Electrohydrodynamic Patterning of Nanocomposites. Goldberg Oppenheimer, P., Eder, D. and Steiner, U. Advanced Functional Materials, 21(10): 1895-1901, 2011.

6. Rapid Electrohydrodynamic lithography using low viscosity polymers. Goldberg Oppenheimer, P., and Steiner, U. Small, (6): 248-1254, 2010.

7. Preparation and characterization of a novel pyrrole-benzophenone copolymerized silica nanocomposite as a reagent in a visual immunologic-agglutination test. Goldberg Oppenheimer, P., Cosnier S, Marks RS and Regev, O. Talanta, 75 (5): 1324-1331, 2008.

8. Exploring a nanotube dispersion mechanism with gold-labeled proteins via cryoTEM imaging. Pola Goldberg-Oppenheimer and Oren Regev. Small, 3(11):18941899, 2007.

9. Proton enriched high-surface area cesium salt of phosphotungstic heteropolyacid with enhanced catatlytic activity fabricated by nanocasting strategy. Madhusudhan Rao, P., Goldberg Oppenheimer, P., Kababya, S., Vega, S. and Landau MV. Journal of Molecular Catalysis A: Chemical, (275): 214-227, 2007. 


\section{Contents}

1 Introduction 1

1.1 "From Patterns to Patterning" . . . . . . . . . . . . . . . . . 1

1.2 Instability-Induced Polymer Patterning . . . . . . . . . . . . . . . . 4

1.3 Outline of the Thesis . . . . . . . . . . . . . . . . . 7

2 Theoretical Background and Physical Principles of EHD Instabilities 11

2.1 Fundamental Concepts of Polymers . . . . . . . . . . . . . . . 11

2.2 Stability of Thin Homogeneous Polymer Films . . . . . . . . . . . . . . 14

2.2.1 Hydrodynamics . . . . . . . . . . . . . . . . . . 16

2.2 .2 Interfacial Pressure . . . . . . . . . . . . . . . . . . . . . 19

2.2.3 Linear Stability Analysis and Dispersion Relation . . . . . . . . 26

2.3 Electrohydrodynamic Instabilities in Thin Polymer Films . . . . . . . . 28

2.3.1 Electrohydrodynamics . . . . . . . . . . . . . . 28

2.3.2 EHD Patterning Induced by a Homogeneous Electric Field . . . 28

2.3.3 EHD Lithography Induced by a Laterally Varying Electric Field 29

3 Experimental Tools and Analytical Techniques 31

3.1 Overview of the Experimental Procedure . . . . . . . . . . . . . . . . 31

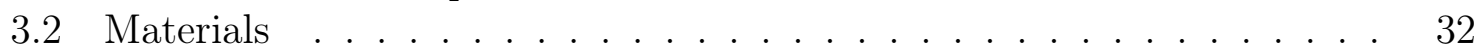

3.2.1 Substrates . . . . . . . . . . . . . . . . . 32

3.2.2 Patterned Materials . . . . . . . . . . . . . . . . . . 32

3.2 .3 Solvents . . . . . . . . . . . . . . . . . 33

3.3 Sample Preparation . . . . . . . . . . . . . . . . . . . 34

3.3.1 Thin Metal Film Evaporation on Substrates . . . . . . . . . . 34

3.3.2 Cleaning of the Substrates . . . . . . . . . . . . . . . . . . 34

3.3.3 Snow-Jet Cleaning . . . . . . . . . . . . . . . 35

3.3.4 Surface Energy Modification . . . . . . . . . . . . . . 35

3.3.5 Thin Film Deposition: Spin-Coating Process . . . . . . . . . . . 36

3.4 Characterization Techniques . . . . . . . . . . . . . . . . 38

3.4.1 Optical Microscopy . . . . . . . . . . . . . . . . . . . . 39

3.4.2 Atomic Force Microscopy . . . . . . . . . . . . . . . . . . . . . . 40

3.4.3 Scanning Electron Microscopy . . . . . . . . . . . . . . . . . . . 42

3.4.4 Transmission Electron Microscopy . . . . . . . . . . . . . . . 43

3.4.5 Surface Enhanced Raman Scattering . . . . . . . . . . . . . . 45

4 Rapid Patterning of Low-Viscosity Resists Using Electrohydrodynamic Lithography

4.1 Introduction . . . . . . . . . . . . . . . . . . . . . . . . 49

4.1 .1 Stability Analysis . . . . . . . . . . . . . . . 51

4.2 Experimental . . . . . . . . . . . . . . . . . . 54

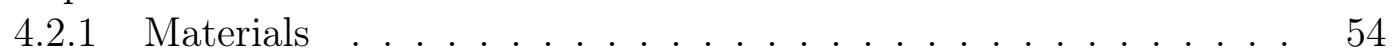

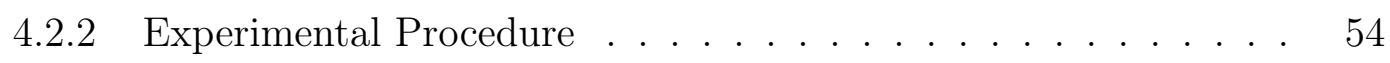

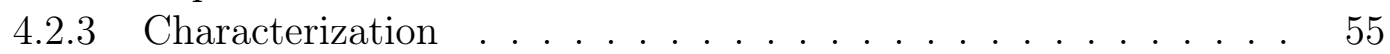

4.3 Results and Discussion . . . . . . . . . . . . . . . . 56 
4.3.1 Homogeneous Electric Field Induced EHD Pattern Formation in Low-Viscosity Materials . . . . . . . . . . . . . .

4.3.2 EHL Pattern Replication of Low-Viscosity Resists in a Heterogeneous Electric Field . . . . . . . . . . . . . . . 57

4.3.3 Current Monitoring of the Rapid EHL Process . . . . . . . . . . 58

4.3.4 Variation of the Instability Time Constant with the Viscosity . 60

4.4 Conclusions . . . . . . . . . . . . . . . . . . . . 62

5 Alignment of Carbon Nanotubes via EHD-Driven Patterning of Nanocomposites

5.1 Introduction . . . . . . . . . . . . . . . . 63

5.1.1 Carbon Nanotubes: Structure and Properties . . . . . . . . . 63

5.1.2 EHD Lithography: A Route Towards Well-Defined CNT Structures in a Patterned Nanocomposite . . . . . . . . . . . . . 65

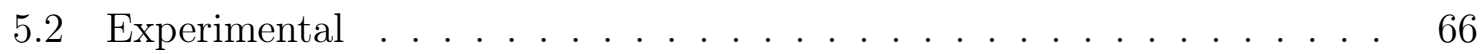

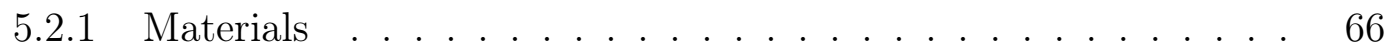

5.2.2 Experimental Procedure . . . . . . . . . . . . . 67

5.2 .3 Characterization ................... 67

5.3 Results and Discussion . . . . . . . . . . . . . . . . . . 68

5.3.1 Dispersion of CNTs and Their Incorporation Into the Polymer Matrix . . . . . . . . . . . . . . . . . . . 68

5.3.2 EHD Patterning of the CNTs-Based Polymer Nanocomposite . . 69

5.3.3 Preferential MWCNT Alignment Within the EHD Generated Structures . . . . . . . . . . . . . . . . 72

5.3.4 Mechanism Leading to the CNTs Anisotropy . . . . . . . . . . . 74

5.3.5 Electrical Properties of the Aligned CNT-Nanocomposites . . . 74

5.3.6 Potential Uses of CNT-Brushes . . . . . . . . . . . . 75

5.4 Conclusions . . . . . . . . . . . . . . . . . . . . . 76

6 Hierarchical EHD Structures for Surface-Enhanced Raman Scatter$\begin{array}{ll}\text { ing } & \mathbf{7 8}\end{array}$

6.1 Introduction . . . . . . . . . . . . . . . . . . . . . . . . . . . . . . . . . . .

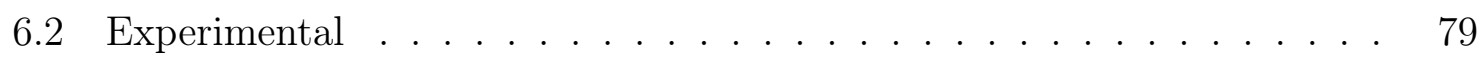

6.3 Results and Discussion . . . . . . . . . . . . . . . . 81

6.3.1 Principles of Bespoke HEHD Pattern Formation Process . . . . 81

6.3.2 Variation of SERS Surface Enhancement and Reflectance with Aspect Ratios of Primary Pillars . . . . . . . . . . . . . . 84

6.3.3 Generation of Hierarchical EHD Architectures . . . . . . . . . 85

6.3.4 HEHD Formed Patterns as Advanced Substrates for SERS . . . 87

6.3.5 Conclusions . . . . . . . . . . . . . . . 88

7 Patterning of Crystalline Organic Materials via EHL 89

7.1 Introduction . . . . . . . . . . . . . . . . . . 89

7.2 Experimental . . . . . . . . . . . . . . . . . . . . . . . . . . . . 91

7.3 Results and Discussion . . . . . . . . . . . . . . . . . . 92

7.3.1 EHL of Crystalline Polymers . . . . . . . . . . . . . . 92 
7.3.2 EHD-Induced Crystallization _. . . . . . . . . . . . . 93

7.3.3 Possible Mechanism for the Crystal Orientation . . . . . . . . . 95

7.4 Conclusions . . . . . . . . . . . . . . . . 103

8 Electrohydrodynamic Lithography of a Conducting Polymer 104

8.1 Introduction . . . . . . . . . . . . . . . . . . 104

8.2 Experimental . . . . . . . . . . . . . . . . . 106

8.3 Results and Discussion . . . . . . . . . . . . . . . . . . 107

8.3.1 Principal of EHD Patterning of Thin Leaky Dielectric Films . . 107

8.3.2 EHL Generated Conductive Patterns Exhibit a Range of Feature

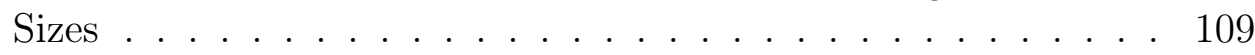

8.3.3 Tuning the Structural Morphology . . . . . . . . . . . . . 111

8.4 Conclusions . . . . . . . . . . . . . . . . . . . . 111

9 Structural Hierarchy of Functional Block Copolymer System Induced $\begin{array}{lr}\text { by Electrohydrodynamic Lithography } & 113\end{array}$

9.1 Introduction . . . . . . . . . . . . . . . . . . . . . . . . 113

9.2 Experimental . . . . . . . . . . . . . . . . . . . . 115

9.3 Results and Discussion . . . . . . . . . . . . . . . . . 117

9.4 Conclusions . . . . . . . . . . . . . . . . . . . . . 125

$\begin{array}{ll}10 \text { Summary } & 126\end{array}$

$\begin{array}{ll}\text { Bibliography } & 131\end{array}$ 


\section{Introduction}

\section{1 "From Patterns to Patterning"}

We understand the word "pattern" as related to a set of recurring elements or events reiterating in a periodic and predictable manner. We know how to recognize a pattern, how to appreciate the aesthetics of a recurring pattern for instance, in a piece of art, we know how it feels to sense a pattern with our hands, how the periodic pattern of wind sounds when we drive through a tunnel with an open window. We can use any of our five senses to directly experience patterns.

Patterns are an integral part of our everyday lives, and they may be found nearly anywhere: in nature, in art, in drama, in mathematics, in imaging, in computers or in science and in many more contexts. Patterns can range from simple decorative polka dots or zigzags, to rather intricate designs such as coast lines and tree structures, which repeat their shape regardless of the viewing magnification, and constitute a part of mathematical patterns named fractals. Mathematics itself is commonly described as the "science of pattern". Any sequence of numbers that may be modeled by a mathematical function is considered a pattern. Another common use of patterns is in the JPEG compressed image format. An image is typically divided into a grid pattern of equal-size tiles, and then each tile is analyzed independently in order to identify the dominant patterns in that part of the image contained by the individual tile. As more compression is applied, the best-match tiles are chosen from a smaller set of available tiles. The patterns found in nature are particularly intriguing. Surface properties are largely determined by the surface energy, but also by the surface structure. Intricate, hierarchical structures found in certain living creatures are responsible for fascinating properties and features such as iridescence, camouflaging or improved fluidics while spanning many length scales. For instance self-cleaning leaves in nature are based on random patterns comprised of microscopic wax crystals. Periodic arrays in butterflies' wings (Fig. 1.1C) lead to colourful optical properties and their iridescent surfaces help them to elude potential predators. The skeleton of a sponge called Venus's flower basket (Euplectella aspergillium) (Fig. 1.1A) constructs for itself an intricate, hierarchical cage 
of silica with each element composed of smaller elements, and at each scale size there are details of design, range of patterns and construction. Another example is the structural hierarchy of the geckos (Fig. 1.1B) which is responsible for their incredible adhesive system. Geckos have millions of dry adhesive hairs (setaes) with mesoscale arrays of setae-bearing scansors found on their feet. The microscale array of setae are arranged in a nearly grid-like pattern on the ventral surface of each scansor. An additional internal nanoscale array consists of hundreds of spatular tips of a single gecko seta, allowing them to adhere to nearly any surface. These designs in nature are as elegant as anything engineers can conceive and their fabrication, particularly for creatures such as the almost brainless sponge faintly resembling and without no raw materials other than seawater to work with, is way beyond the best that engineers or scientists can do.
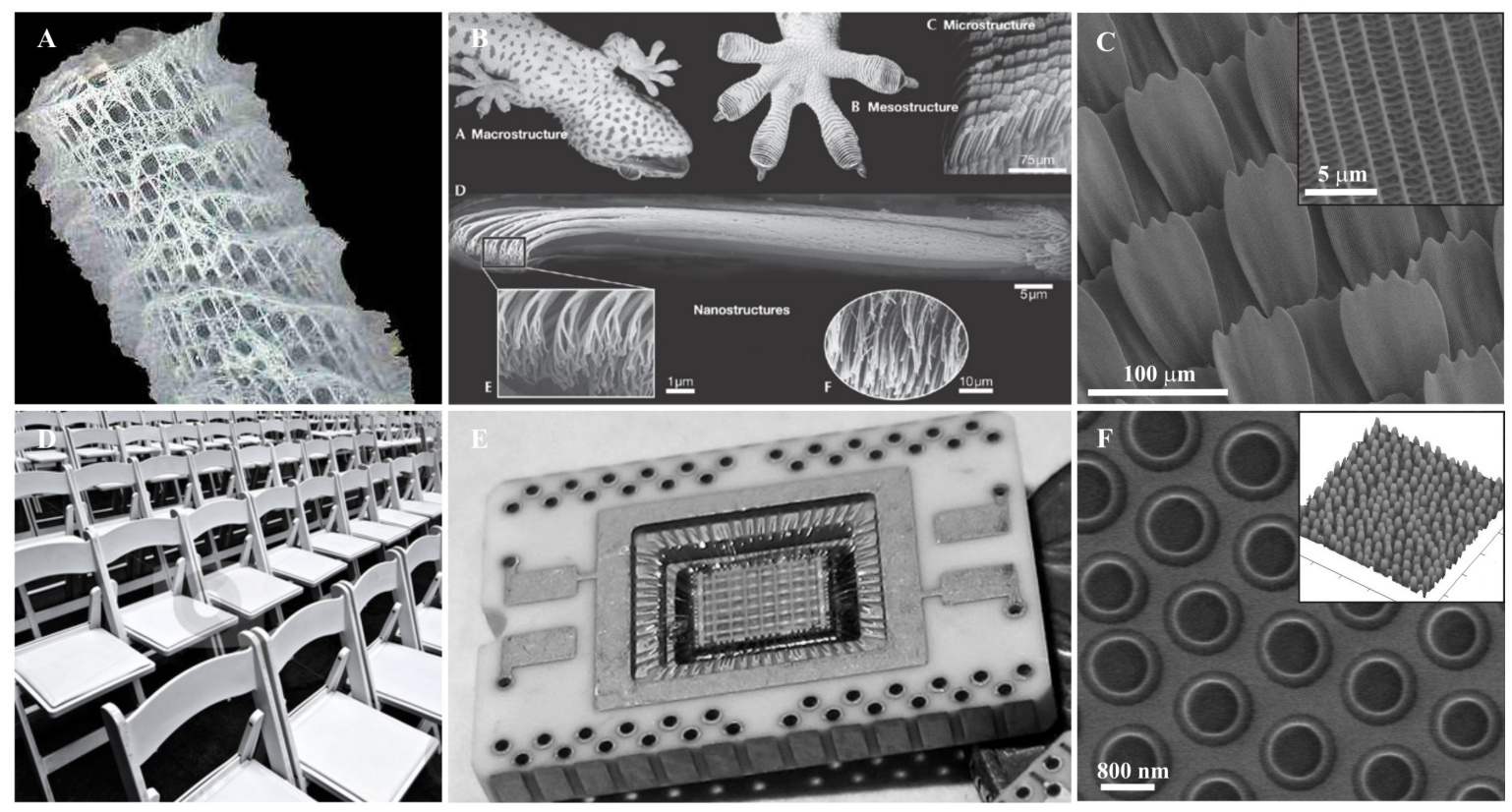

Figure 1.1: Patterns in nature and technology. (A). Spectacular structure of a glass sponge also known as Venus' Flower basket. Adopted from [58]. (B). Structural hierarchy of the gecko adhesive system. Adopted from [119].(C). Scanning electron microscopy (SEM) images of microstructured butterfly wings. The butterfly wings have iridescent surface, helping them to elude potential predators. Courtesy of J. J. Rickard. (D). Rows of chairs creating a macroscale pattern or a template. Adopted from [1]. (E). Typical CCD device divided into 56 rectangular sectors, each filled with a fine tracery of the integrated circle material. Adopted from [164]. (F). Representative top view SEM and three dimensional atomic force microscopy (inset) patterns of pillars with hexagonal symmetry generated by electrohydrodynamic patterning technique.

In technology, structured films are often used in sensors but their most abundant use is in the semiconductor industry, where thin polymer films provide the medium by which patterns are transferred from a structured mask into the semiconductor. These form the most important basic step in the fabrication of microelectronic devices. In 
our everyday lives therefore, we rely to a major extent on the extensive exploitation of predominantly (invisible) submicron patterns. The majority of medical, securityaimed, micro and opto-electronic devices consist of patterned thin films. The rapid pace of advancements in our technological era requires a continual decrease in the size of features of devices, such as computer chips, solid state memory, sensors and holograms. Inspired by macro-patterns, and in particular, by nature's staggering designs in the past decade, science and technology have been experiencing the germination of new methods and fabrication technologies for micro and nano patterning which are essential for enabling a variety of advanced applications.

"Patterning", on a macro level, can be described using a simplified image of ordered chairs as seen in Fig. 1.1D. When chairs are regularly spaced, and point in the same direction, they invite and orient a milling crowd to take their seats say for a wedding, a play, a graduation. As everyone takes their seat a pattern is set. In a similar manner, patterning strategy applied on several tens of magnitude smaller lengths scales is used to form, for instance, the intricate traceries of wires and transistors that comprise the microelectronic chips (Fig. 1.1E). The most conventional micro-to-nano patterning methods typically exploit light, electrons or ions. Other methods rely on mechanical processes to induce patterns on both rigid and flexible substrates with different surface geometries. Polymers are often used for surface patterning. The diversity, the relatively low cost, the convenient mechanical properties and the compatibility with most patterning techniques render polymers attractive candidates for patterning processes. Polymer-patterned surfaces are expected to enable a broad range of new technologies. These include: the fabrication of light-emitting diodes (LEDs), semiconductor microelectronics and plastic electronics; $[15,140]$ the fabrication of masks and templates; $[56,153]$ bio-inspired and medical devices including the study of cells and tissue engineering; [64, 105] the production of optical components such as gratings or photonic crystals [161] and fundamental research in surface science and combinatorial synthesis. $[39,135]$

The control of patterns on sub-micrometre lateral length scales is of considerable technological interest, and is especially relevant for tailoring the properties of novel functional materials. A variety of bottom-up and top-down patterning techniques for patterning polymers have emerged in the past decade. The most prominent examples include photolithography, laser writing, electron beam lithography, soft lithography imprinting, self-assembly of block copolymers, molding or embossing-based techniques and holographic patterning. [18, 24, 62, 93, 177, 178] Hitherto, however, most of the existing strategies for patterning polymers have revealed limitations in certain aspects. Among the difficulties are those related to resolution, position control, physical limits, versatility and reproducibility. In addition the attempts to pattern polymers seem still 
to have insufficient throughput for commercial applications.

Downsizing of patterns' critical dimensions aimed for technologically appealing down-scaled devices has been and still is the driving force behind the development of novel lithographic techniques. However, depending on the application the requirements for optimal patterning process may vary. While the vast majority of microto-nano fabrication techniques utilize resists of macromolecular nature, the extension towards additional material systems such as glassy, ceramic, ferroelectric and crystalline materials is of major importance for enabling invaluable surface functionalities that can not be accomplished with polymers alone. Moreover, for many applications it is desirable to control the spatial arrangement of more than one component. With traditional methods the process requires an iterative, multistep, procedure, rendering the patterning process intricate and less reproducible. The growing demand for better performance, reduced energy consumption, high-throughput and higher levels of complexity and integration raise the need for alternative technologies capable of more than the generation or reproduction of patterns below the sub-100 nm. Successful cost-effective patterning processes are needed to enable straightforward high-fidelity patterning in a controlled manner on multiple-scales which will also be suitable for a broad range of materials. Development of such methods is essential for the successful future large scale fabrication of advanced devices.

One of the alternative and somewhat less established lithographic techniques uses soft polymeric materials to (re)produce complex patterns into a thin polymer film by exploiting surface instabilities. The research described in this thesis focuses on the unconventional patterning of multiple materials through utilization of the destabilization of a polymer film by means of an electric field, in order to produce a wealth of structures spanning many length scales (i.e., ranging from $20 \mu \mathrm{m}$ to $100 \mathrm{~nm}$ ).

\subsection{Instability-Induced Polymer Patterning}

The stability of liquid films is known to have many important applications. Spontaneously developing instabilities in thin films, on the other hand, can be harnessed to produce novel structures with technologically interesting length-scales. Consequently, instabilities in thin films have been the focus of recent research and are of practical relevance for a variety of applications.

Temperature gradients or electric potentials applied perpendicularly to thin, liquid films were used to produce modulations in surface tension, buoyancy or charge density. [16] Above the threshold of instability, such systems generated a range of lateral periodic patterns such as hexagons, rings, spirals and squares. While yielding largescale ordered chemical or topographic patterns, upon reduction of the temperature or 
evaporation of a solvent, dynamic structures generated in liquid polymers or monomers could be frozen-in and investigated. $[82,180]$ Such surface-tension-driven or buoyancydriven convection patterns generated in films of liquid monomers subjected to vertical temperature are used in basic studies of convection in complex fluids. Research has focused on the prediction of patterns periodicity as a function of temperature gradients and macroscopic properties of liquids.

In another instability-driven approach to polymer patterning, application of a high $\left(10^{8} \mathrm{~V} / \mathrm{m}\right)$ electric field across thin polymer films at temperatures above the glasstransition temperature of the polymer generated variations in charge density, destabilized the film, and created lateral and topographic features on the film. [128-131] The emerging ordered patterns are a direct result of the interaction of a vertically applied electric field with a flow of fluid. Hence, this has been termed the electrohydrodynamic (EHD) patterning process. The experimental investigation of polymer pattern formation under the influence of an electric field was first developed by E. Schäffer et al. [130] In these experiments, researchers showed that in a confined geometric space with an applied external voltage and a small inter-electrode spacing, the dielectric is polarized, resulting in an effective force on the interface. While assuming a perfect dielectric film and by employing linear stability analysis, the most unstable modes as set by the competition between the electrostatic forces and surface tension were identified. The force imbalance selected a wavelength which was amplified to the greatest extent. The amplitude of this mode increased and has begun to dominate the system, until eventually its maxima touched the upper electrode to form a hexagonal array of liquid pillars. This generated pattern exhibited a well defined lateral wavelength while spanning the gap between the two electrodes. Patterns of hexagonal arrays of liquid pillars could be obtained by using two planar electrodes (Fig. 1.1F). By using a topographically patterned electrode, the periodicity of lateral features of the pattern was reduced from $10 \mu \mathrm{m}$ to $140 \mathrm{~nm}$, while more complex shapes of the polymer structures were reproduced. In these cases, the instability was directed towards the protrusions of the template facing a liquid polymer film, thereby reproducing the mask pattern.

The dynamics of pattern formation has been modeled using non-linear numerical simulations [162, 173] and linear stability analysis, [107, 108, 130, 131, 176] which has described the early stages of amplification of film undulations. The later stage of the pattern-formation process was qualitatively determined by the destabilizing force (e.g., electric field), whereas for the electrostatically unfavourable dielectric interfaces the initial undulations developed towards a liquid morphology spanning the two electrodes. [166] Furthermore, Pease and Russel developed the leaky dielectric model [106] which allowed for redistribution of charges at the interfaces, and predicted both reduced spacing and faster growth rates than those predicted for the perfect dielectric. Wu et 
al. [174] demonstrated that proper design of the mask can overcome the limitation of the natural domain size and achieve ordered patterns over large areas on the basis of EHD patterning. Their work has shown that both narrow ridges as well as trenches on a patterned mask can guide the development of pillars at desired locations. Verma et al. [162] employed non-linear 3D simulations to demonstrate that the pattern-replication mechanisms depend on a number of parameters, such as the degree of mismatch between the template periodicity and the intrinsic instability wavelength, the ratio of the film thickness to the air gap and the magnitude of the lateral field variation. Subsequently, Voicu et al. [166] presented an experimental study which complemented the results of Verma's simulations. D. Barbero also studied the destabilization process of thin liquid polymer films subjected to an electric field, providing complementary information about the hydrodynamics of the liquid films. His work proposed a model for the disentanglement of large macromolecules during the formation of thin films by spincoating providing an explanation for the higher mobility observed in thin liquid layers.

In the published literature, EHD lithography was used predominantly for the patterning of high-molecular weight, amorphous polymers of a dielectric nature. Surface functionalities of structured materials are of crucial importance for a range of applications. Polymer based materials alone cannot always fulfil these requirements. Other conductive, crystalline, or composite materials often offer extra functionalities and values. Furthermore high molecular weight polymers have a much higher viscosity in the liquid state compared to lower molecular weight polymers. Thin film instabilities therefore develop much more slowly in viscous films in comparison to low viscosity liquids. Therefore, the EHD patterning is hindered by the slow replication dynamics requiring many hours for the pattern formation to complete. Moreover, hierarchy in structure formation is essential for many applications requiring cost-effective patterning on multiple length scales on the same sample.

The work described in this thesis exploits the electrohydrodynamic patterning technique to generate a wealth of structures on sub-micrometer length scales from a broad range of materials, while significantly reducing the patterning time to technologically appealing rates. The method, initially developed by E. Schaffer and U. Steiner, fundamentally explored by D. Barbero and extended by N. Voicu, is taken several steps further in this study, presenting the technological appeal of the technique with advanced, practical applications. Thin films of low-viscosity materials, crystalline and conductive polymers, carbon-nanotubes-based polymer composites, (semi)conductive blockcopolymers and their combinations in multilayer systems are effectively patterned, yielding useful, intricate hierarchical architectures and functional structures with simultaneously induced anisotropy inside the electrohydrodynamically driven micro-andnano-patterns. The applied potential of generated patterns with novel functionalities 
is explored, verified and confirmed. Achievement of a balance between high speed and high resolution, and a low-cost patterning process without compromising pattern performance renders this method and the according surface structures as complementary candidates to many conventional lithographic techniques and as such, imperative candidates for a range of applications.

Intriguingly, EHD-based lithography, while founded on a simple concept with a rather generic experimental principle, has been expanded to become a pathway for further possibilities and applications. Last, but not the least, the aesthetic beauty of the generated structures and the simple theories based on basic principles render this field of research appealing, intriguing and gratifying.

\subsection{Outline of the Thesis}

Following this introduction, Chapter 2 lays out the theoretical background pertaining to polymeric liquids and the stability of thin liquid films. First, a brief historical overview of polymers and an introduction to polymer properties is presented. Subsequently, the effect of surface tension and van der Waals' forces are discussed and the ways in which additional external forces influence the stability of thin films is addressed. The hydrodynamic equations under the constraint of volume conservation are solved within a linear stability analysis and under the lubrication approximation. The resulting dispersion relation provides a generic equation for the most unstable mode in a system. Finally, the mechanism by which EHD film destabilization in the presence of external electrostatic pressure evolves into well-defined patterns is discussed.

Chapter 3 includes a general description of the experimental setup, as well as the materials used in this work, cleaning procedures and the experimental details of the sample preparation. The characterization techniques that were used to analyze topography and characteristic length scales of patterns formed in thin films along with their applications are explained.

Each of the following six chapters is devoted to a different material or set of materials patterned via EHD method, followed by the discussion of the unique outcomes, functionalities and the extent of the control accomplished with an outlook towards authentication of potential applications.

Chapter 4 describes the background, theory and experimental results for the rapid electrohydrodynamic lithography using low-viscosity polymers. After an introduction and descriptions of the experimental details, the stability analysis is summarized in brief, with the resulting equations normalized to dimensionless quantities revealing important consequences and trends of low-viscosity EHD patterning. An improved monitoring system essential for the rapid pace of EHD pattern formation is introduced. The 
leakage current across the device is monitored and the sigmoidal shape of the current curve is correlated with the various stages of EHD pattern formation. A discussion on the accelerated mode of electrohydrodynamic lithography as a promising prospect for the rapid, straightforward, and low-cost replication of sub-micrometer patterns follows.

Chapter 5 deals with the EHD pattern formation in carbon nanotube-polymer composite films. First, an optimized dispersion and embedding of randomly oriented carbon-nanotubes (CNTs) in polymer matrix is presented. Subjection of this nanocomposite to the EHD patterning is shown to give rise to well-defined microscale columnar patterns with embedded CNTs which are uniaxially aligned parallel to the cylinder axis. Conductive pathways in nanotube networks formed during EHD patterning of nanocomposite films result in a substantial increase in the composites' conductivity at loadings exceeding the percolation threshold. The ability to control and manipulate the position of the formed patterns as well as the alignment of the incorporated CNTs using EHD lithography is discussed. Finally, generation of surfaces decorated by CNTbrushes is accomplished suggesting a possibility for establishing adhesive conducting electrical contacts, and, paving the way for integration of nanotube-based composites into functional devices.

In Chapter 6 a novel route for the fabrication of multi-scale three-dimensional structured arrays as surfaced enhanced Raman scattering (SERS)-active platforms is introduced. While most SERS substrates are typically manufactured by conventional lithographic methods, the developed cost-effective approach to create nanostructured surfaces provides addressable alternative for the SERS community. Herein, a method to create controlled self-organized hierarchical nanostructures using electrohydrodynamic (HEHD) instabilities is described. HEHD pattern formation harnesses a sequential instability in multilayer thin films induced by an electric field to guide the layer material into design structures, allowing different materials to be patterned in a one-step procedure. The created structures are shown to be fine-tuned, thus fulfilling an important requirement for SERS. Each of the HEHD patterned individual structural units yield a considerable SERS enhancement. The different generated structures display plasmonic properties which are controlled by the HEHD patterning parameters. In this chapter, the fabrication of the HEHD morphologies, each of which can be effectively tailored to provide high SERS enhancement, to the best of our knowledge, described and applied in SERS for the first time.

Chapter 7 presents a straightforward route to pattern semi-crystalline organic materials. Herein, EHD lithography is shown to enable simultaneous structuring of organic crystalline materials while aligning the chains with respect to the formed structures. The exposure of liquified films to a heterogeneous electric field gives rise to submicron sized patterns which can be lithographically controlled. Possible mechanisms inducing 
the vertical $c$-axis of the crystal lattice in EHD patterned films are also discussed. Structured crystalline polymer materials with anisotropic molecular orientation are of great interest in various applications, because of their special optical and electronic properties. Because of the generic nature of the experimental approach, the results of this chapter suggest that a broad spectrum of crystalline materials can be patterned by using this technique. A successful combination of bottom-up and top-down approaches based on the electrohydrodynamic generation of surface patterns in thin crystalline films with the uniaxially aligned crystals constitutes a promising route for the generation of functional sub-micrometer structures that were hitherto mainly accessible through rather cumbersome and expensive techniques.

The potential technological application is explored in Chapter 8, where a conductive polymer is patterned for the first time using the EHD patterning method. This process is shown to provide a single-step and cost-effective approach for direct patterning of conjugated polymers on solid substrates, generating variety of feature sizes ranging from tens of micrometer to hundreds of nanometers. The physical mechanism of the EHD pattern formation process in the case of a leaky dielectric is described, discussing the modifications in the electric field. Conductive Polypyrrole is synthesised and employed during EHD patterning process providing a proof-of-concept and opening a window for potential applications for the developments in the fields of an advanced microelectronics, flexible display devices and sensors.

The last experimental assembly explored in this study is described in Chapter 9, introducing an additional system with an applied technological potential. This chapter describes the fabrication of hierarchical structures spanning three several length scales and demonstrating a combined bottom-up and top-down approach based on EHD formation of patterns in fully functional block copolymer films and the self-assembly of block copolymer within the formed patterns. Confining electro-conductive block copolymer (with donor and acceptor blocks) in presence of the electric field into fabricated microstructures gives rise to an internal vertical alignment of the copolymers nanometric domains with an additional molecular orientation. Inside the superstructure films ordered arrays of nanocrystals of the constituting block are aligned in smectic phase altering the linearly polarized light. Controlled alignment of the nanodomians is shown to considerably improve the charge conduction toward the device electrodes. The advantages and higher registration achieved during the alternative use to the thermal annealing of a solvent-vapour atmosphere during patterning of functional block copolymers is also discussed. These findings evolve as very promising to be truly exploited in optoelectronic and photovoltaic devices.

Finally, the thesis concludes with Chapter 10 summarizing the experimental outcomes and applications. The intriguing aspects of physics and technology of instabilities 
in thin polymeric films are restated, while also relating to some of the unanswered questions. The thesis concludes with a section relating to the prospects for the future of the EHD technique and its applied potential. 


\section{Theoretical Background and Physical}

\section{Principles of EHD Instabilities}

\subsection{Fundamental Concepts of Polymers}

The availability of materials has been of a considerable impact on the human development over many centuries. Ever since the Stone age, through the Bronze and Iron ages and to the current age emerging as a "Polymer age", humans have been constantly surrounded by polymers wherever they might be. The naturally occurring biopolymers, the DNA, the proteins and starches in foods, the tires on our bikes and cars, cotton, wool and rubber, are only a number of examples of polymers, which are supreme in their diversity and properties.

A polymer is a chemical structure formed by polymerization, the process by which elementary units, monomers are covalently bonded together. The name is derived from the Greek, ( $\pi \mathrm{o} \lambda v$, polu)-(many) and ( $\mu \varepsilon \rho \mathrm{o} \zeta$, meros)- (parts) and refers to molecules consisting of many repeating monomers. If all the monomer segments are the same, it is called a homopolymer, if different, a copolymer. The total numbers of structural units, $N$, is the degree of polymerization. Typically, the outcome of the polymerization process is a mixture of macromolecules with a range of molecular weights, $M_{\mathrm{w}}$ or polydispersity which is one of the distinguishing features of most synthetic polymers and is described by its molecular weight distribution. The molecular weight of monomer units, $M_{i}$ is typically on the order of 100 while the total weight $M_{\mathrm{w}_{i}}=N_{i} M_{i}$ can range from 1000 to above $10^{6}$. The conventional way to describe the molar mass of a polymer chain is the mass of one mole (equal to Avogadro's number) of these molecules. The most common units of molar mass are $\mathrm{g} / \mathrm{mol}$, since, in those units the numerical value (i.e., average molecular weight multiplied by Avogadro's number) equals to the average molecular mass in atomic mass units. The weight distribution is given by the ratio of the total weight of the polymer to the number of molecules and is described by the number average molecular weight, $\bar{M}_{\mathrm{n}}$

$$
\bar{M}_{\mathrm{n}}=\frac{\sum_{i=1}^{\infty} M_{\mathrm{w}_{i}}}{\sum_{i=1}^{\infty} N_{i}}
$$


where $N_{i}$ is the number of chains with mass $M_{i}$. The average molecular weight where $M_{\mathrm{w}}$ obtained by randomly choosing the monomer is

$$
\bar{M}_{\mathrm{w}}=\frac{\sum_{i=1}^{\infty} M_{\mathrm{w}_{i}} M_{i}}{\sum_{i=1}^{\infty} M_{\mathrm{w}_{i}}}=\frac{\sum_{i=1}^{\infty} M_{i}^{2} N_{i}}{\sum_{i=1}^{\infty} M_{i} N_{i}}
$$

The summation $\sum_{i=1}^{\infty}$ is a notation for a sum over all possible values of structural units where $i=N$. The polydispersity index, $P_{\mathrm{d}}$ is defined as the ratio of the weightto number average molecular weights $M_{\mathrm{w}} / M_{\mathrm{n}}$ and is equal to unity for monodisperse polymers. A larger value of $P_{\mathrm{d}}$ corresponds to polymers with broader molecular weight distribution.

The physical properties of polymeric systems vary and mainly depend on microscopic structure, which is the arrangement of molecules in the bulk. Polymers can exhibit a randomly arranged amorphous structure with no order, or a semicrystalline structure with partial organization in ordered crystalline regions, called lamellae. In lamellar part of the chains are packed parallel in a crystalline structure, coexisting with amorphous regions between these lamellae. On larger length scales this layered morphology is organised in spherical regions, the spherulites (Fig. 2.1).

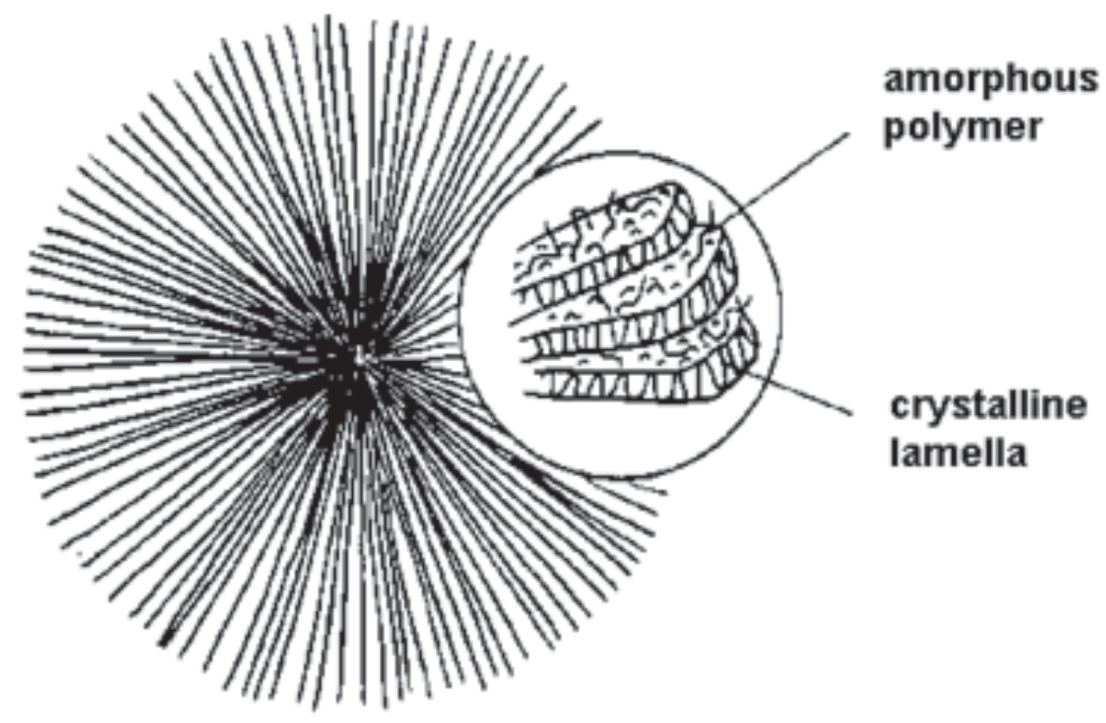

Figure 2.1: Crystallization of polymer melts yields semicrystalline materials comprised of folded chains in lamellae which are packed into a larger spherulitic structure, while coexisting with amorphous regions. Adopted from [3].

Polymer solutions are obtained by dissolving a polymer in a solvent. In solution polymer chains can adopt number of configurations depending on the interaction with the solvent. If these are weak the polymer assumes the shape of a random coil with a Gaussian density distribution. At high enough concentrations parts of the coils 
interpenetrate since the chain cannot distinguish between a neighbor and itself. An important measure of the spatial extent of the coil is its radius of gyration, $R_{\mathrm{g}}$

$$
R_{\mathrm{g}}=\frac{l \sqrt{N}}{\sqrt{6}}=\frac{l \sqrt{M_{\mathrm{w}} / M_{\mathrm{i}}}}{\sqrt{6}}
$$

where $l$ is the effective segment length.

A bulk liquid state of a polymer melt is formed at high enough temperatures when no solvent is present. In the melt, depending on their intermolecular interactions, polymers can be categorized as glassy, semicrystalline, elastic, or viscous. The viscous state, consists of a liquid of macromolecules that are free to move. When this motion is hindered by the presence of cross-links, polymers can only rearrange on length scales smaller than the mean distance between cross-links, and the material is therefore, elastic. Upon cooling, the polymer melt can either transform into a semicrystalline solid below its melting temperature, $T_{\mathrm{m}}$, or into a polymeric glass below its glass transition temperature, $T_{\mathrm{g}}$. A first order phase transition, $T_{\mathrm{m}}$ defines the transition from the liquid to crystalline phase and is characterized by discontinuities in the heat capacity and specific volume. While isotactic homopolymers with regular configurations crystallize readily, atactic polymers with random stereochemistry possess some intrinsic disorder that prevents crystallization, and thus, upon cooling (below the polymers' $T_{\mathrm{g}}$ ) transform into a more brittle amorphous glassy state. In most of the experiments described herein, polymers are in their glassy state at room temperature. At temperatures above their $T_{\mathrm{g}}$ the segments of the chains become free to move and polymers can be described as incompressible viscous fluids. At the transition temperature from melt to glass, the specific volume varies non-linearly with temperature, but, unlike crystallization, it is continuous. Even though upon cooling or heating through the glass transition range, the material exhibits considerable changes in physical properties, including a change in a heat capacity, there is no latent heat involved. The nature of the glass transition is a subject of continuing research.

Liquid polymers, in comparison to low molecular weight molecules, have a much higher viscosity. Low molecular liquids are often Newtonian fluids with constant viscosities as a function of the flow rate. The viscosity of polymer melts and concentrated solutions is non-Newtonian as it does not show the linear behavior. In the experiments described in this thesis, the melt viscosity $\eta$ is taken in the limit of zero-shear, or no flow velocity: $\eta=\eta_{0}$. The zero-shear melt viscosity scales linearly with the molecular weight below the entanglement molecular weight, $M_{\mathrm{e}}$, where the polymer forms a temporary network of entangled chains. The zero-shear melt viscosity scales with $M_{\mathrm{w}}$ as $\eta \propto M_{\mathrm{w}}^{3.4}$.

Polymeric liquids have a microstructure which resembles springs representing the 
linear chain. However, polymeric fluids are not ideal elastic materials, and they also have a dissipative reaction to deformation, the viscous dissipation. Thus, at the one end of the frequency spectrum polymeric liquids behave according to Newtonian fluids mechanics, and on the other, according to Hookean elasticity. The viscoelastic (nonNewtonian) behaviour is inherent to all polymers and can be understood in terms of the reptation model. One can easily pull on the end of one polymer coil and it leaves behind a tube, defining the only direction along which the polymer is free to reptate. The time necessary for the molecule to leave this tube defines the reptation time $\tau_{\text {rep }}$. While for times longer than $\tau_{\text {rep }}$, the polymer has lost its memory of the constraining tube and is free to move in any direction, for times shorter than $\tau_{\text {rep }}$, the chain is confined to the tube under the influence of Brownian motion, and can only respond elastically.

\subsection{Stability of Thin Homogeneous Polymer Films}

The fundamental understanding along with an ability to fine-tune the physical properties and behavior of thin polymer films are indispensable for a large number of applications in research and industry.

Usually, instabilities in thin films are undesirable in technology. Defect-free smooth films in the form of insulating layers or photoresists, coatings, lubricants or protective layers are essential for most important applications and in particular, in the microelectronics. The practical importance of stable homogeneous films for a variety of technological applications along with the scientific interest in the interactions of liquids near surfaces, have driven the effort to understand the stability of thin liquid films. The dynamics of liquid films have therefore been the subject of many theoretical [121, 162, 176] and applied studies. [13, 53, 127, 129, 135] A typical thin film is a liquid layer on a substrate with a (free) surface where the liquid is exposed to air (or a gas) or another fluid. Film deposition techniques, such as spin-coating, dip coating or vapor deposition, are used to prepare a thin film on a substrate. Thin polymer films supported on solids substrates exhibit a different behavior relative to both the bulk and the thick films (i.e., films that are thicker than the capillary length). The possibility of an ideal bulk configuration is hindered by the confinement of the polymer chains at the film interfaces. In a confined medium, a film is essentially 2 Údimensional, and is different from the bulk. New effects related to the geometry may arise. When the polymer film is laterally confined, it adjusts to the imposed boundary conditions. Liquids in a confined geometry behave differently from those in large volumes. This is due to the dominance of the liquids surface and interfacial tension over other body and surface forces at length scales below the capillary length. In the capillary regime, 
liquid morphologies are dominated by a minimization of the overall surface free energy. A second aspect where the confinement of a liquid plays a role concerns the stability of thin films. While planar liquid films are intrinsically stable, they can for instance be destabilized by a van der Waals pressure. In the process of film destabilization, the driving force couples to the spectrum of capillary waves and a single capillary mode with a well-defined wavelength develops.

The stability of a thin film is determined by the transient behavior of undulations on its surface. Liquid surfaces are never completely flat. A spectrum of capillary waves is always present caused by the Brownian motion of the molecules. If additional forces couple to this initial capillary wave spectrum, fluctuations might either be amplified or damped. Interactions such as gravity, surface-tension forces, van der Waals forces, externally applied forces such as electric or magnetic fields or temperature gradients acting on the interface yield stresses at the interface and generate film instabilities. The precise control of instabilities in films can however, be utilized to produce novel structures by exploiting the destabilization of an initially homogeneous layer in a spontaneous structure formation process, at technologically interesting length scales. Since, instabilities externally induced by electrostatic field are the focal point of this study and are extensively covered in the following chapters, it is essential to take a closer look at the process governing dynamic instabilities of thin liquid films to gain more comprehensive understanding of the physical mechanism(s) behind this process.

The generic experimental system is depicted in Fig. 2.2. A thin polymer film (with initial thickness $h_{0}$ is deposited onto a silicon substrate and liquified by annealing above its $T_{\mathrm{g}}$. The liquid is described as an incompressible (i.e, constant density, $\rho$ ) viscous fluid (in the zero frequency limit the liquid is Newtonian, i.e. the shear force per unit area is proportional to the local velocity gradient; the proportionality constant is the viscosity, $\eta$ ). [77] The thickness of the film $h$ is commonly much smaller than the lateral

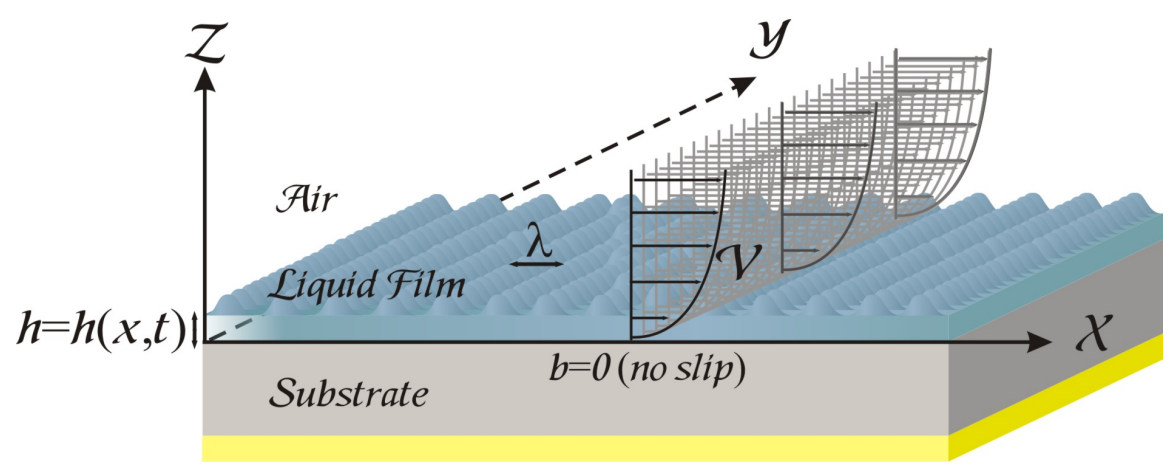

Figure 2.2: Schematic representation of a thin supported polymer film with thickness $h$. Temperature fluctuations induces capillary surface waves with wavelength $\lambda$. The Poiseuille-type flow is indicated by a parabolic flow profile with velocity $v$. 
extent of the film $L$, and as a result, the flow of liquid primarily takes place in the lateral direction (parallel to the substrate). The $z$-coordinate of the film surface is given by $h=h(x, t)$ where $x$ is the lateral coordinate, $t$ is the time. A spectrum of capillary waves is formed at the liquid surface due to the molecular motion of the fluid at finite temperatures. Capillary waves are perturbations of the free interface and generate flow in the film which can be described using a hydrodynamic approach. Calculating the evolution of a capillary wave spectrum is based on assuming a sinusoidal fluctuation with wave number $q=2 \pi / \lambda$ and amplitude $\zeta\left(\zeta \ll h_{0}\right)$

$$
h(x, t)-h_{0}=\operatorname{Re}\left\{\zeta e^{i q x+t / \tau}\right\}
$$

The dispersion relation relates the time constant $\tau$ to each $q$ and establishes whether fluctuations with wavelength, $\lambda\left(\lambda \gg h_{0}\right.$, the long wavelength limit relative to the intial film thickness $h_{0}$ ) are exponentially amplified or suppressed. The modulation of the free interface determines liquid material transport in the plane of the film which is described by the Navier-Stokes equation and which yields the velocity profile, $v$ in the film. In combination with an equation of continuity for the system, the hydrodynamics of the interface can be derived.

\subsubsection{Hydrodynamics}

The motion of an incompressible Newtonian fluid with viscosity $\eta$ is fully described by classical hydrodynamic theory [77] for known external pressure, $p$ and an average velocity, $\bar{v}$. The Navier-Stokes (N-S) equation of motion for an incompressible fluid is based on Newton's second law stating that an infinitesimal volume element moving with the fluid is accelerated by the forces acting on it. Since the thickness of the film is much smaller than the lateral extent of the film and the flow of liquid primarily takes place in the lateral direction, the explicit vector equation for the $x$ coordinate is given by:

$$
\rho\left(\frac{\partial v}{\partial t}\right)+\rho\left[u\left(\frac{\partial v}{\partial x}\right)+v\left(\frac{\partial v}{\partial y}\right)+w\left(\frac{\partial v}{\partial z}\right)\right]=-\frac{\partial p}{\partial x}+\eta\left(\frac{\partial^{2} v}{\partial x^{2}}+\frac{\partial^{2} v}{\partial y^{2}}+\frac{\partial^{2} v}{\partial z^{2}}\right)+\rho g
$$

where the velocity components $u, v, w$ are the dependent variables to be solved for and $g$ is the gravitational acceleration.

The left hand side of a N-S equation is a product of fluid acceleration $\rho(d v / d t)$ and a convective term $\rho(v \cdot \nabla v)$ which renders the hydrodynamic equation non-linear. The right hand side of the equation is the total force balance that acts on the fluid element. The forces stem from the pressure gradient at the free interface, the viscous force $\eta \nabla^{2} v$ 
resulting from the momentum transfer from faster to slower moving layers in the fluid and the gravity $(\rho g)$. For flow in thin films, the N-S equation can be simplified in the following way:

1. Since the high viscosities of polymer melts result in a low flow velocity, the quadratic terms in Navier-Stokes equation can be neglected: $u\left(\frac{\partial v}{\partial x}\right)+v\left(\frac{\partial v}{\partial y}\right)+$ $w\left(\frac{\partial v}{\partial z}\right) \rightarrow 0$

2. Since the resulting dynamics are slow, the velocity profile can considered to be always in a quasi-steady-state: $\rho\left(\frac{\partial v}{\partial t}\right) \rightarrow 0$

3. For thin-films with dimensions smaller compared to the capillary constant (i.e., $a=\sqrt{2 \gamma / g \rho})$, gravity does not have an influence on the shape of the interface: $\rho g \rightarrow 0$

4. For $\lambda \gg h_{0}$ and $\zeta \ll h_{0}$ : it is possible to approximate the flow in the film as steady laminar flow caused by a pressure gradient in the lateral, $\mathrm{x}$-direction and the velocity gradient which varies only along the $z$-axis.

In order to calculate the dominant wavelength of the instabilities, it is sufficient to simplify the equations to only one lateral coordinate. Additionally, the fluctuations have a small amplitude compared to the film thickness. The further analysis is restricted to wavelengths large compared with the depth of the liquid.

The above considerations yield the simplified Navier-Stokes equation along the $x$ and $z$ coordinates:

$$
\begin{gathered}
0=-\frac{\partial p}{\partial x}+\eta\left(\frac{\partial^{2} v}{\partial z^{2}}\right) \\
\left(\frac{\partial^{2} v}{\partial z^{2}}\right)=\frac{1}{\eta} \frac{\partial p}{\partial x} \\
\frac{\partial p}{\partial z}=0
\end{gathered}
$$

Since the pressure is constant within the depth of the film (along $z$ ), the pressure gradient is a function of $x$ only, meaning that the pressure gradient along $x$ is constant. Therefore, integration of this differential equation with respect to $z$ leads to a parabolic velocity profile:

$$
v(z)=\frac{1}{2 \eta}\left(\frac{\partial p}{\partial x}\right) z^{2}+c_{1} z+c_{2}
$$

To calculate the velocity, the boundary conditions related to the values of stresses at the substrate-liquid and liquid-air interfaces must be known. At the substrate in the lubrication approximation [101] a non-slip boundary condition is typically assumed 
$v(z=0)=0$. At the liquid-air interface $(z=h)$ the stress within the fluid is counterbalanced by stresses arising from the surfaces forces. In terms of equation of continuity this boundary condition can be expressed as

$$
\eta_{\text {liq }} \frac{\partial v_{\text {liq }}}{\partial z}=\eta_{\text {air }} \frac{\partial v_{\text {air }}}{\partial z}
$$

Since, there are no stresses in the air (gas), the sum of the viscous and the surface stresses is zero, and the stress at the surface vanishes.

$$
\eta_{\mathrm{liq}} \frac{\partial v_{\mathrm{liq}}}{\partial z}=0=\sigma_{\mathrm{xz}}
$$

Using the boundary conditions:

1. Zero slip: $v(z=0)=0 c_{2}=0$

2. No-lateral stresses at the film surface: $v(z=h) \sigma_{\mathrm{xz}}=\eta \frac{\partial v}{\partial z}=0$ yields the constants:

1. $c_{1}=-\left.2 \frac{\partial p}{\partial x} y\right|_{y=h}=2 \frac{\partial p}{\partial x} h$

2. $c_{2}=0$, and the velocity profile is obtained:

$$
v(z)=\frac{1}{2 \eta}\left(\frac{\partial p}{\partial x}\right) y(z-2 h)
$$

The mean velocity in the film is:

$$
\bar{v}=\frac{1}{h} \int_{0}^{h} v(z) d z=\frac{h^{2}}{3 \eta}\left(-\frac{\partial p}{\partial x}\right)
$$

This equation describes a Poiseuille type flow in the liquid film $\mathrm{n}$ the presence of a pressure gradient. The minus sign indicates that the flow is in the direction of the decreasing pressure. In the lubrication approximation, for the one dimensional (1D) case the lateral Poiseuille volume flow rate induced in the direction of decreasing pressure through a cross section of the film, $A$ is obtained:

$$
j=\left.A\right|_{A=h} \cdot \bar{v}=h \cdot \bar{v}=\frac{h^{3}}{3 \eta}\left(-\frac{\partial p}{\partial x}\right)
$$

To calculate the interface profile an additional continuity equation is required which is derived from the mass conservation of a small volume element (fixed in space) within an incompressible flowing fluid. For two plane cross-sections through the film a distance $d x$ apart, a volume (per unit time) $(A \bar{v})_{x}$ passes through one and $(A \bar{v})_{x+d x}$ through the 
other, changes by:

$$
(A \bar{v})_{x}-(A \bar{v})_{x+d x}=-\frac{\partial(A \bar{v})}{\partial x} d x
$$

Then, the change in volume (due to the variation of incompressible fluid level) per unit time through the film can directly be represented by: $\frac{\partial(\text { volume })}{\partial t}=\frac{\partial A}{\partial t} d x$ which corresponds to $\frac{\partial A}{\partial t} d x=-\frac{\partial(A \bar{v})}{\partial x} d x$, yielding the required equation of motion for the 1-dimensional case $(A=h$ and $j=A \bar{v})$ that includes the volume conservation:

$$
\frac{\partial h}{\partial t} d x+\frac{\partial(A \bar{v})}{\partial x} d x=0=\frac{\partial h}{\partial t}+\frac{\partial j}{\partial t}
$$

Following this any change in the height of the film results in a flow in the lateral direction redistributing the fluid from the valleys to the peaks of the undulations. Substitution of the equation of flux into the equation of continuity yields the final form of the equation of motion for the film surface:

$$
\frac{\partial h}{\partial t}=\frac{\partial}{\partial x}\left[\frac{h^{3}}{3 \eta} \frac{\partial p}{\partial x}\right]
$$

\subsubsection{Interfacial Pressure}

The interfacial pressure acting at the surface separating the two media must be determined in order to calculate the dynamics of the liquid-air interface. The total interfacial pressure is uniform across the depth of the film. However, the pressure does depend on the film thickness. It can be written as sum of several contributions:

$$
p(h)=p_{0}+p_{\mathrm{vdW}}(h)+p_{\mathrm{L}}(h)+p_{\mathrm{ex}}(h)
$$

where $p_{0}$ is the ambient air pressure which is independent of the film thickness for $h<$ capillary length. The second term are the van der Waals (vdW) attractive or repulsive intermolecular forces which act between all atoms and molecules. $p_{\mathrm{L}}(h)$ is the Laplace pressure stemming from the curvature of the interface and $p_{\mathrm{ex}}(h)$ can be any excess surface pressure which in this thesis is the electrostatic pressure induced by externally applied electric field, $p_{\mathrm{el}}(h)$ ) enabling the electrohydrodynamic (EHD) pattern formation process. In the following paragraphs, the vdW, Laplace and excess pressures are each discussed shortly, eventually establishing the most significant contributions to the total interracial pressure during the EHD patterning process. 


\section{Laplace Pressure}

A thoroughly investigated instability is related to destabilizing molecular forces. Destabilizing molecular interactions lead to film rupture if these are sufficiently strong to overpower the stabilizing surface tension. The latter opposes film thickness fluctuations in very thin liquid film. The surface tension $\gamma$ is defined as the excess free energy at the liquid-air interface that stems from the imbalance in the attractive forces experienced by molecules at the surface of the liquid. To minimize the interfacial energy, a geometric shape with the highest possible volume to surface ratio is formed. Surface tension is the direct outcome of the cohesive forces holding the liquid molecules together. Various phenomena such as capillarity, the shapes of macroscopic liquid droplets on surfaces and the contact angle between coalescing soap bubbles arise from the surface energy, $\gamma$ of solids and liquids (for a liquid, $\gamma$ is usually referred to as its surface tension. Surface tension is the free energy change when the surface area of medium is increased by a unit area. A free liquid will always tend to minimize its surface energy by minimizing its surface area.
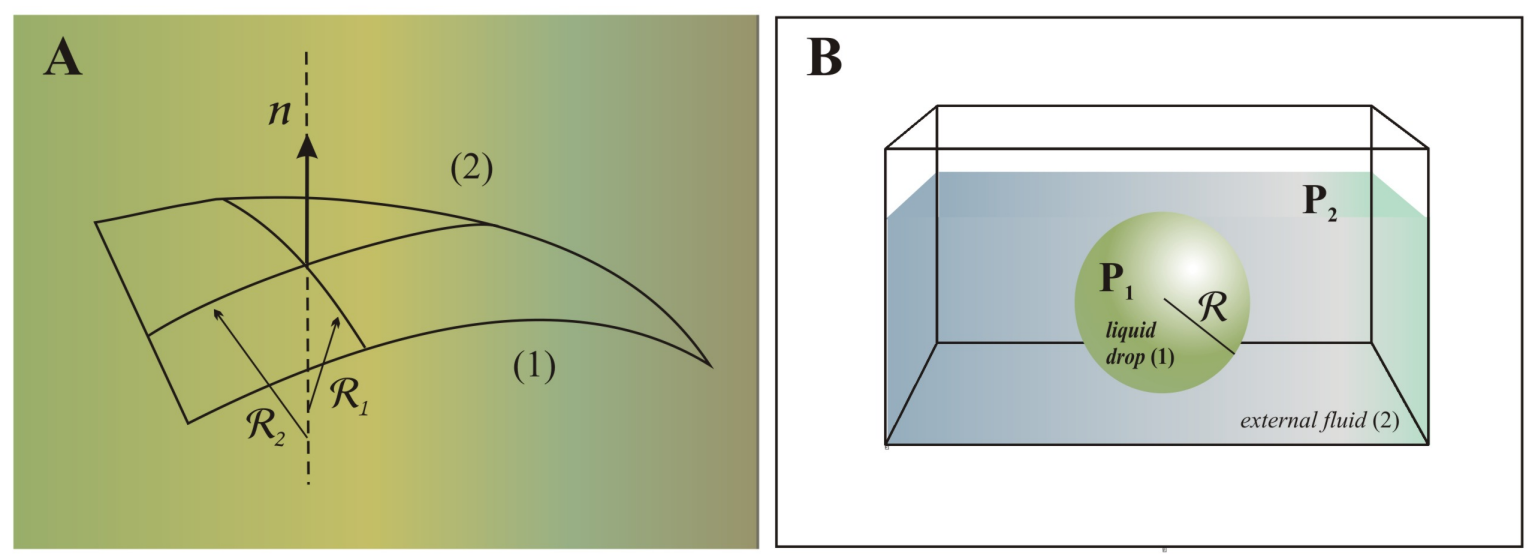

Figure 2.3: (A). Illustration of the definition of the principal radii of curvature $R_{1}$ and $R_{2}$ for a random geometry boundary surface between fluids (1) and (2). (B). Calculation of Laplace pressure: considering a 3D spherical drop (1) immersed into a liquid (2), its equilibrium is achieved when the pressure inside the drop, $P_{1}$ exceeds the pressure outside $P_{2}$ by: $p_{1}-p_{2}=p_{\mathrm{L}}=\gamma / R$.

A curved liquid surface, which locally increases the surface area, generates a restoring pressure difference, i.e., Laplace pressure across the interface, which is inversely proportional to the radii of curvature and scales with the surface tension

$$
p_{1}-p_{2}=\gamma_{\mathrm{L}}\left(\frac{1}{R_{1}}+\frac{1}{R_{2}}\right)=p_{\mathrm{L}}
$$

such that the planes defining the radii of curvature, $R_{1}$ and $R_{2}$ are perpendicular to each other (Fig. 2.3), and are necessary to describe a curved surface in three dimensions. 
This equation relates the pressure difference (i.e., Laplace pressure) across the interface to the radius of curvature and the surface tension. In the absence of any other pressure, Laplace pressure always stabilizes the film by damping out all perturbations. For the one dimensional case, the radius of curvature is given by $1 / R=-\partial^{2} h / \partial x^{2}$, yielding:

$$
p_{\mathrm{L}}=-\gamma \frac{\partial^{2} h}{\partial x^{2}}
$$

For a flat surface the $R \rightarrow \infty$ and there is no pressure difference across a planar boundary.

\section{van der Waals Pressure}

The stability of very thin $(h<10 \mathrm{~nm})$ liquid films is dictated by the effective molecular interactions between the substrate and the film arising from van der Waals forces. In very thin polymer melt films, vdW interactions are dominant in determining the stability of coatings. [135] These ever present forces, play a role in a host of various phenomena such as surface tension, adhesion and the structure of condensed macromolecules such as proteins and polymers.

Collective, long-range van der Waals forces consist of three important interactions: Keesom orientation (i.e., Boltzmann averaged interaction between two permanent dipoles), Debye induction (i.e., interaction between a polar and a non-polar molecule) and the dispersion force (i.e., interaction between neutral molecules) that together contribute to the total vdW interaction between atoms and molecules. Since, they are always present, independent on the properties of the molecules, dispersion forces also known as induced dipole-induced dipole forces make the most important contribution to the vdW interactions between apolar molecules.

Dispersion forces are quantum-mechanical in origin and amenable to a host of theoretical treatments of varying complexity. Intuitively, they can be understood in terms of an electrostatic interaction arising from the dipole fluctuations of two neighboring atoms or molecules. For a non-polar atom at any given instance, the instantaneous positions of the electrons induce a finite dipole moment which in turn generates an electric field that polarizes neighboring neutral atoms. This induced dipole-induced dipole interaction gives rise to an attractive force between two identical atoms. If two atoms are a substantial distance apart, continuously fluctuating original dipole may has already changed its orientation, and the interaction between them becomes smaller. With distance, this retardation effect changes the strength of the van der Waals interaction and its scaling behavior.

For an interatomic vdW pair potential it is possible to sum the energies of all atoms in one body with all the atoms in the other and thus, obtain the "two-body" potential 
for two surfaces. The resulting interaction is given in terms of the Hamaker constant, $A$, which quantifies the magnitude of the force and depends on the electronic structure of the involved materials through the density and the strength of the oscillating dipoles. If the Hamaker constant is negative resulting in repulsive van der Waals forces, the film minimizes its free energy by increasing its thickness while wetting the surface and is therefore stable; if the Hamaker constant is positive which means attractive vdW forces, the film reduces its free energy by thinning, and it is unstable and dewets. vdW forces play an eminent role in thin films and the pair-wise integration of all dipole-dipole interactions results in an effective force, which can be either attractive or repulsive depending on the dielectric properties of the film and the bounding materials. The vdW adhesive pressure for the non-retarded interaction of a medium 3 sandwiched between two media 1 and 2 is given as

$$
p_{\mathrm{vdW}}=-\frac{A_{132}}{6 \pi h^{3}}
$$

To obtain the Hamaker constant for any system it is essential to know how the dielectric permittivity of every medium varies with frequency, requiring access to the entire electromagnetic frequency spectrum, which is typically not covered by the experimental data. Approximating the Lifshitz calculation, Tabor and Winterton derived a simplified model for calculating the Hamaker constant. [150] However, if the adsorption frequencies of all three media are assumed to be the same, while ignoring molecular rotational frequency, $\nu_{\text {rot }}$ and considering a main contribution from the electronic UV absorption, $\nu_{\mathrm{e}}\left(\nu_{\mathrm{e}}=3 \times 10^{5} \mathrm{~s}^{-1} \gg \nu_{\mathrm{rot}}\right)$, an approximate expression can be obtained for the non-retarded Hamaker constant: $A_{\text {tot }}=A_{\nu=0}+A_{\nu>0}$

$$
\begin{aligned}
A_{\text {tot }} & \approx \frac{3}{4} K T\left(\frac{\varepsilon_{1}-\varepsilon_{3}}{\varepsilon_{1}+\varepsilon_{3}}\right)\left(\frac{\varepsilon_{2}-\varepsilon_{3}}{\varepsilon_{2}+\varepsilon_{3}}\right) \\
& +\frac{3 h_{\mathrm{P}} \nu_{\mathrm{e}}}{8 \sqrt{2}} \frac{\left(n_{1}^{2}-n_{3}^{2}\right)\left(n_{2}^{2}-n_{3}^{2}\right)}{\sqrt{n_{12}+n_{32}} \sqrt{n_{22}+n_{32}}\left(\sqrt{n_{12}+n_{32}}+\sqrt{n_{22}+n_{32}}\right)}
\end{aligned}
$$

where $\varepsilon_{1}, \varepsilon_{2}$ and $\varepsilon_{3}$ are the static dielectric constants of the three media, $n_{1}, n_{2}$ and $n_{3}$ are the corresponding refractive indexes $\left(\varepsilon=n^{2}\right)$, and $h_{\mathrm{P}}$ is Plank's constant. The first term gives the zero-frequency energy of the vdW interaction and includes the Keesom and Debye dipolar contributions. The second term is the dispersion energy. Since $h \nu_{\mathrm{e}} \gg k T$, the dispersive part usually dominates over the dipolar contribution unless the refractive indices of two of the involved materials are similar. The zerofrequency contribution therefore often only accounts for a few percent of the total magnitude of the Hamaker constant. Since it does not depend on the frequency, it is 
not affected by any retardation. The cross-over from non-retarded to retarded forces occurs typically at film thicknesses on the order of $10 \mathrm{~nm}$, resulting in the vdW force:

$$
\Phi_{\mathrm{vdW}}=\frac{B_{132}}{h^{4}}
$$

where $B$ is the retarded Hamaker constant.

\section{Excess Surface Pressure}

$p_{\text {ex }}$ is the energy necessary per unit area to bring two interfaces from infinity to a certain distance $h$. It includes any excess surface pressure, and is typically called the conjoining pressure $\Pi$ (the opposite of the disjoining pressure). $\Pi$ is the pressure required to pull the film boundaries, i.e., disjoining them. It is defined as

$$
\Pi=-p_{\mathrm{ex}}=-\frac{\partial \Phi}{\partial h}
$$

where, $\Phi$ is the excess free energy per unit area, also named effective interface potential. The disjoining pressure is usually used for the repulsive interactions in the context of wetting films.

In the absence of any externally applied fields, $p_{\mathrm{ex}}$ is equivalent to the disjoining pressure caused by the dispersive vdW interactions. For thin films with only nonretarded vdW forces, with the interface potential (adhesion energy)

$$
\Phi_{\mathrm{vdW}}=-\frac{A_{132}}{12 \pi h^{2}}
$$

When an electric field is externally applied across the liquid polymer-air interface, it polarizes the polymer, it induces an effective surface charge density. [85, 130, 131] In this case the dominant external pressure is the destabilising electrostatic pressure $p_{\mathrm{ex}}=p_{\mathrm{el}}$.

\section{Electrostatic Pressure}

\section{Perfect Dielectric}

The core of this thesis is the EHD patterning technique which exploits surface instabilities induced by an external electric field. The physical principles of the EHD method are discussed in detail in section 2.3 .

The overall pressure distribution at constant temperature in the film for dominant electrostatic interactions is given by 


$$
p(h)=p_{0}+p_{\mathrm{L}}(h)+p_{\mathrm{el}}(h)
$$

For a sufficiently strong electric field in a capacitor, the electrostatic interactions much stronger than the van der Waals interactions. This means that in electric field experiments the dispersive interactions are only a small correction. Therefore, the vdW pressure can be neglected.

For a perfect dielectric liquid polymer in a capacitor an applied potential difference across the two electrodes (at distance $d$ ) gives rise to an electric field, $E_{\mathrm{f}}$ across the dielectric material. The electric field causes the energetically unfavorable build-up of displacement charges at the dielectric interface, resulting in an effective surface charge density. The charges at the polymer-air interface experience an effective attraction with the oppositely charged upper electrode. Thus, the electric field acts to align the interface separating the two media parallel to the electric field lines while minimizing the free electrostatic energy of the system. The strong electrostatic pressure at the liquidair interface overcomes the stabilizing effects of the surface tension and destabilizes the film:

$$
p_{\mathrm{el}}=-\frac{1}{2} \varepsilon_{0} \varepsilon_{\mathrm{p}}\left(\varepsilon_{\mathrm{p}}-1\right) E_{\mathrm{f}}^{2}
$$

$\varepsilon_{\mathrm{p}}$ is the dielectric constant of the polymer and $\varepsilon_{0}$ is the dielectric permittivity of the vacuum. The origin of the destabilizing $p_{\mathrm{el}}$ is schematically shown in Figure 2.4. $E_{\mathrm{f}}$ can be calculated from the free energy, $F$ stored in the capacitor with a constant applied voltage, $U$

$$
F=F_{0}-\frac{1}{2} C U^{2}
$$

where $C$ is the capacitance, and $F_{0}$ is is the free energy in the absence of an applied voltage. The variation of the free energy with respect to the film thickness, $h$ per unit area of the interface, gives the interfacial electrostatic pressure

$$
p_{\mathrm{el}}=\frac{1}{A} \frac{\partial F}{\partial h}=-\frac{U^{2}}{2 A} \frac{\partial C}{\partial h}
$$

The polymer-air double layer in the capacitor can be modeled as two capacitors in series (Fig. 2.4), one filled with the liquid polymer and the other with air

$$
\frac{1}{C}=\frac{1}{C_{\text {dielectric }}}+\frac{1}{C_{\text {air }}}=\frac{d-h}{\varepsilon_{0} \varepsilon_{\mathrm{p}} A}+\frac{h}{\varepsilon_{0} \varepsilon_{\mathrm{p}} A}=\frac{\varepsilon_{\mathrm{p}} d-\left(\varepsilon_{\mathrm{p}}-1\right) h}{\varepsilon_{0} \varepsilon_{\mathrm{p}} A}
$$


Insertion into Eq. (2.29) for the 1-dimensional case yields

$$
p_{\mathrm{el}}=-\frac{1}{2} \frac{\varepsilon_{0} \varepsilon_{\mathrm{p}}\left(\varepsilon_{\mathrm{p}}-1\right) U^{2}}{\left[\varepsilon_{\mathrm{p}} d-\left(\varepsilon_{\mathrm{p}}-1\right) h\right]^{2}}
$$

A comparison with Eq. (2.27), gives the electric field in the polymer

$$
E_{\mathrm{f}}=\frac{U^{2}}{\varepsilon_{\mathrm{p}} d-\left(\varepsilon_{\mathrm{p}}-1\right) h}
$$

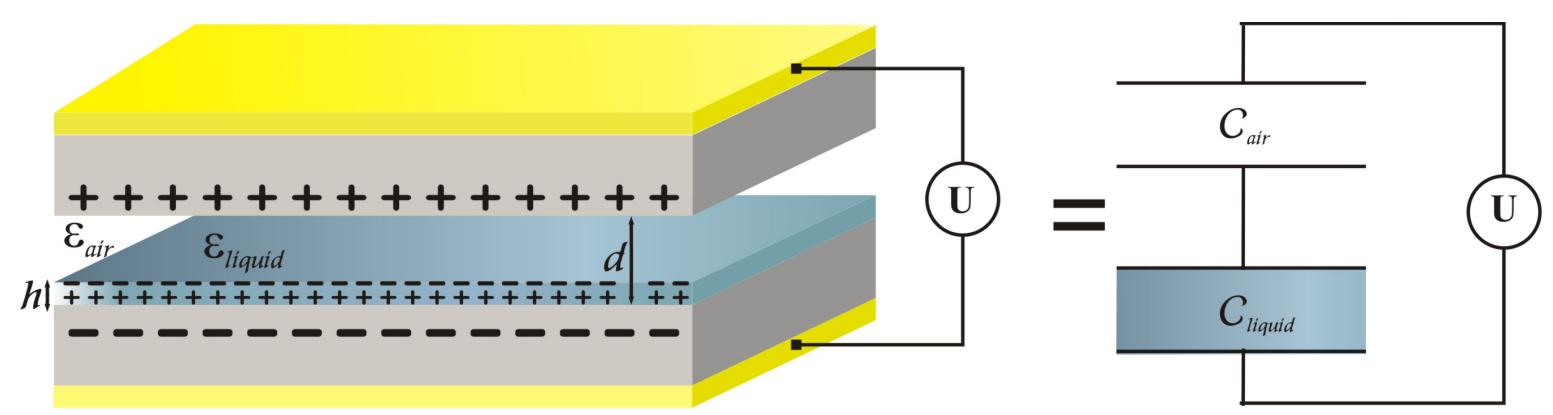

Figure 2.4: An applied electric field leads to the polarization of the dielectric polymer layer, yielding an attractive interaction between the charges at the polymer-air interface with the charges at the upper electrode. The layered system is equivalent to a sum of two capacitors in series.

\section{Leaky Dielectric}

In this thesis several materials were subjected to electric field instabilities during the EHD patterning process. While most of the used materials, (i.e., low-viscosity polymers, nanotube-based polymer composite, crystalline polymers and block-copolymers) are well described by a simple linear stability analysis that models the polymer layer as a perfect dielectric, for EHD patterning of conductive polymers (Chapter 8), free charges at the interface have to be taken into the consideration. A finite polymer conductivity lowers the electric field in the layer and increases the electrostatic stress at the surface, as predicted in the leaky dielectric model by Pease and Russel. [106, 107] The total potential difference spans across the air gap which drives the EHD pattern formation. This is the origin of the instability in the case of a leaky dielectric film.

Similarly to the analysis for the perfect dielectric, the pattern selection of EHD instabilities are given in terms of a linear stability analysis [49, 127] for incompressible, Newtonian fluid assuming the non-slip boundary condition at the substrate. In this case, the destabilising electrostatic pressure is somewhat modified, $U$

$$
p_{\mathrm{el}}=-\frac{1}{2} \frac{\varepsilon_{1} \varepsilon_{0} U^{2}}{\left(d-h_{0}\right)^{2}} .
$$




\subsubsection{Linear Stability Analysis and Dispersion Relation}

The total interfacial pressure consisting of the destabilizing electrostatic pressure and the stabilizing Laplace pressure, $p=p_{\mathrm{el}}+\left(-\gamma \frac{\partial^{2} h}{\partial x^{2}}\right)$ couples to the constantly present capillary waves of the liquid surface, which destabilizes part of the mode spectrum. This is described by the well-established linear stability analysis of a free liquid film, subjected to a pressure $p$, where a sinusoidal form for the film thickness (Eq. 2.4) is used with the temporal evolution of the liquid-air interface governed by equation of motion. Taking the derivative of the equation of motion and inserting the equation for the Laplace pressure gives

$$
\begin{aligned}
\frac{\partial h}{\partial t} & =\frac{h^{2}}{\eta}\left(\frac{\partial h}{\partial x}\right)\left[\frac{\partial p}{\partial x}\right]+\frac{h^{3}}{3 \eta}\left[\frac{\partial^{2} p}{\partial x^{2}}\right] \\
& =\frac{h^{2}}{\eta}\left(\frac{\partial h}{\partial x}\right)\left[-\gamma\left(\frac{\partial^{3} h}{\partial x^{3}}\right)+\left(\frac{\partial p_{\mathrm{el}}}{\partial h}\right)\left(\frac{\partial h}{\partial x}\right)\right] \\
& +\frac{h^{3}}{3 \eta}\left[-\gamma\left(\frac{\partial^{4} h}{\partial x^{4}}\right)+\left(\frac{\partial^{2} p_{\mathrm{el}}}{\partial h^{2}}\right)+\left(\frac{\partial p_{\mathrm{el}}}{\partial h}\right)\left(\frac{\partial^{2} h}{\partial x^{2}}\right)\right]
\end{aligned}
$$

In the long-wavelength limit, where the amplitude of the surface waves are much smaller than the film thickness $\left(\zeta \ll h_{0}\right)$, terms of order $O\left(\zeta^{2}\right) \ll O(\zeta)$, where $\partial h / \partial x \propto$ $\zeta$, all non-linear terms in $\zeta$ are discarded simplifying the above equation to

$$
\frac{\partial h}{\partial t}=\frac{h^{3}}{3 \eta}\left[-\gamma\left(\frac{\partial^{4} h}{\partial x^{4}}\right)+\left(\frac{\partial p_{\mathrm{el}}}{\partial h}\right)\left(\frac{\partial^{2} h}{\partial x^{2}}\right)\right]+O\left(\zeta^{2}\right)
$$

By taking partial derivatives in the above equation and dividing by $\zeta \exp (i q x+t / \tau)$, this coupling of a force balance to the capillary wave spectrum yields a dispersion relation that describes the temporal evolution of the sinusoidal perturbations in $h$ :

$$
\frac{1}{\tau}=-\frac{h^{3}}{3 \eta}\left[\gamma q^{4}+\frac{\partial p_{\mathrm{el}}}{\partial h} q^{2}\right]
$$

The dispersion relation determines whether a wave with a wave vector $q$ is damped or amplified. The stability of the film depends on the exact form of the gradient of the excess surface pressure with respect to the film thickness (see Fig. 2.5): while for $\partial p_{\mathrm{el}} / \partial h \geq 0, \tau<0$ for all $q$ - fluctuations are damped (due to restoring effect of the surface tension), meaning that all growth rates are negative and the film minimizes its surface and is intrinsically stable, for $\partial p_{\mathrm{el}} / \partial h<0$ all modes with $\tau>0$ and $q<$ $q_{c}=\sqrt{-\frac{1}{\gamma} \frac{\partial p_{\mathrm{el}}}{\partial h}}$ are amplified and the film is unstable. For $q>q_{c}$ undulations are 
exponentially damped.

The exponentially fastest-growing mode given by the maximum of the dispersion relation eventually dominates the mode spectrum. It has the wavelength $\lambda=2 \pi / q_{\max }$ :

$$
\lambda_{\max }=2 \pi \sqrt{\frac{2 \gamma}{-\partial p_{\mathrm{el}} / \partial h}}=2 \pi \sqrt{\frac{\gamma U}{\varepsilon_{0} \varepsilon_{\mathrm{p}}\left(\varepsilon_{\mathrm{p}}-1\right)^{2}}} E_{\mathrm{f}}^{-\frac{3}{2}}=2 \pi \sqrt{\frac{\gamma\left[\varepsilon_{\mathrm{p}} d-\left(\varepsilon_{\mathrm{p}}-1\right) h\right]^{3}}{\varepsilon_{0} \varepsilon_{\mathrm{p}}\left(\varepsilon_{\mathrm{p}}-1\right)^{2} U^{2}}}
$$

The associated maximal growth scales with the fourth power of the dominant wave vector, and is proportional to the ratio of surface tension to viscosity for a given $\lambda$ :

$$
\frac{1}{\tau_{\max }}=\frac{\gamma h^{3}}{3 \eta} q_{\max }^{4}
$$

and

$$
q_{\max }=\sqrt{\frac{-\partial p_{\mathrm{el}} / \partial h}{2 \gamma}}=\frac{2 \pi}{\lambda_{\max }}
$$

The two characteristic parameters $\lambda_{\max }$ and $\tau_{\max }$ describe the static and dynamic behavior of the liquid film. While the time constant of the EHD instability can provide rheological information, the characteristic wavelength is representative of the forces acting on the polymer-air interface.

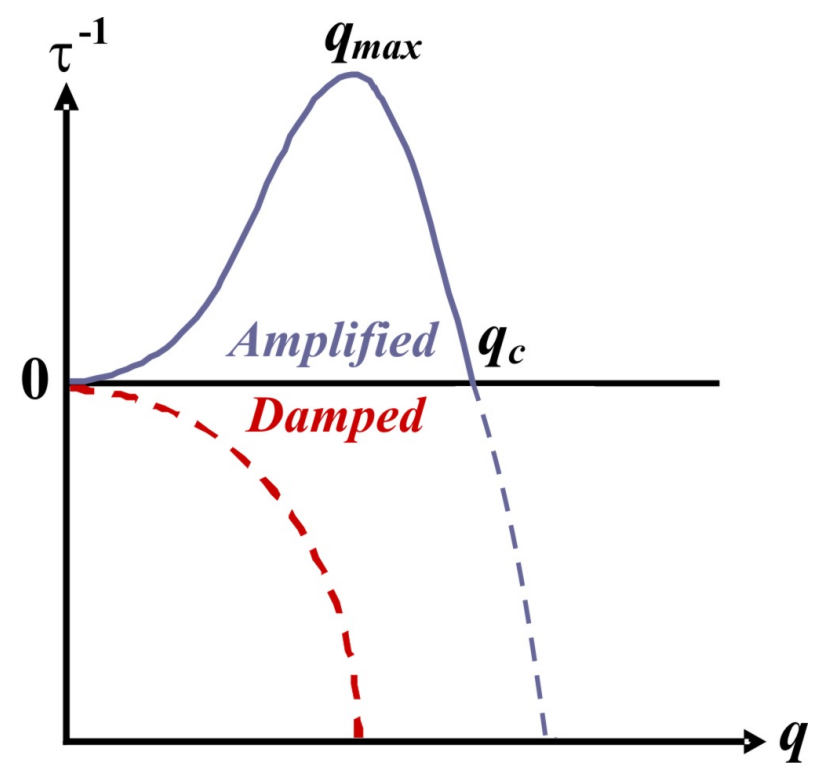

Figure 2.5: Graphic representation of the dispersion relation. While in the absence of an electrostatic pressure, all modes are damped $(\tau<0)$, the dispersion relation yields a dominant mode $q_{\max }$ with a corresponding growth rate $\tau_{\max }^{-1}$. In the region of large values of wave vectors $(\tau<0)$, the liquid increases its surface, to an extent that is energetically unfavourable, resulting in suppression of all small wavelength fluctuations. In the region of large wavelength fluctuations $(\tau>0)$, while the waves are exponentially amplified, the growth rate is limited by the viscous dissipation associated with the in-plane transport of material. 


\subsection{Electrohydrodynamic Instabilities in Thin Poly- mer Films}

\subsubsection{Electrohydrodynamics}

Electrohydrodynamics deals with fluid motion induced by electric fields. The physical principles that describe the electrohydrodynamics, which is the deformation of liquid surface induced by an electric field were first noted in 1897, [149] and followed by the original theories on the electrohydrodynamic instabilities. [41, 159] Ever since these ground-breaking studies, extensive investigation has been carried out on these issues. $[91,92,117,152]$

For macroscopic liquid films and liquid jets systems mentioned hitherto, gravity plays an important role. For thin liquid films below the gravity limiting capillary length, electric fields can be used to destabilize the films in order to form organized patterns. In the capillary regime, the $\gamma$ is the dominant restoring force and opposes the formation of surface fluctuations that increase the surface area. The application of a sufficiently strong $E_{\mathrm{f}}$ to polymer-air or polymer-polymer interface overcomes the surface tension and results in electrohydrodynamic (EHD) instability. The EHD model, that is the basis of the work described in this study, was reported by Schäffer et al. $[127,127,130,130]$ who introduced this electrostatic technique based on an instability which develops in (perfect) dielectric viscous polymer in the presence of an electric field. The generic experimental approach and typical experimental results are shown in Fig. 2.6.

\subsubsection{EHD Patterning Induced by a Homogeneous Electric Field}

A polymer when heated above its $T_{\mathrm{g}}$, begins to flow as a viscous liquid. Consequently, applying an electric field normal to the initially homogeneous (Fig. 2.6A) interface between two dielectric materials with different dielectric constants yields the destabilizing interfacial $p_{\mathrm{el}}$ which overcomes the intrinsic stabilization of the $\gamma$, and couples to the omnipresent part of the capillary wave spectrum, amplifying surface instabilities with a characteristic wavelength, $\lambda_{\max }$ (Fig. 2.6B). A fluid flow with velocity $v$ during the amplification of the initial capillary film undulations leads to a lateral redistribution of the polymer from surrounding thinning regions. These further pinned to the top electrode and detach from the surrounding polymer film by draining the liquid bridge that connects them with the unruptured polymer film. This results in the rearrangement to the energetically favorable configuration of the liquid polymer characterized by an array of pillars with a hexagonal symmetry (Fig. 2.6C) which minimizes the repulsion of the polarized undulations minima and maxima. An inter-pillar distance $\lambda$ is equal 
to the initially dominant and amplified capillary wavelength, and is determined by the field strength and the polymer surface tension. The pillars diameter is a function of the initial film thickness.
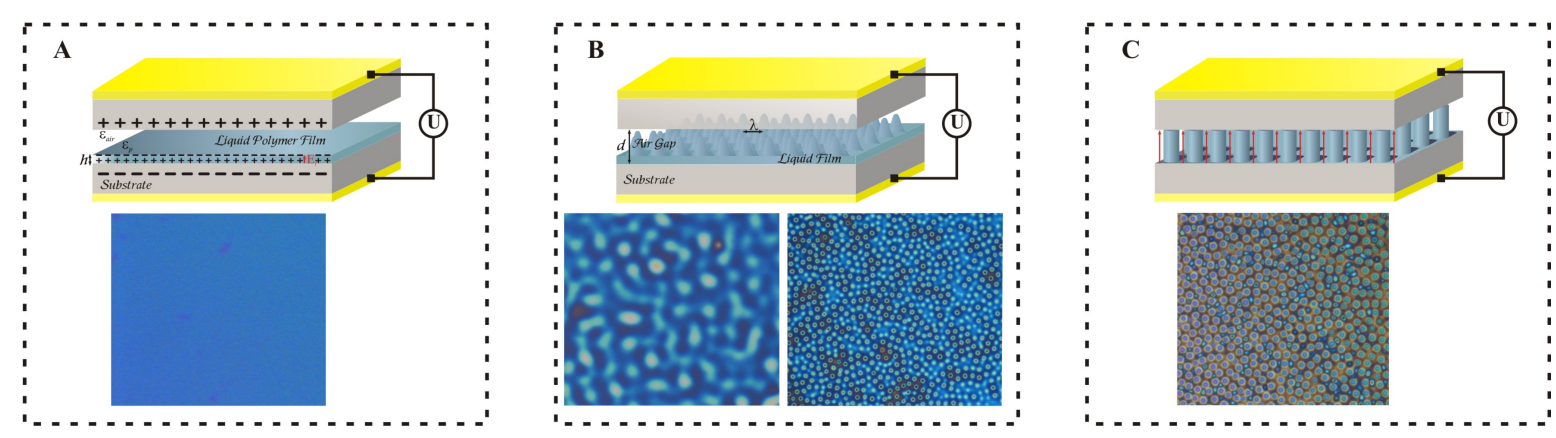

Figure 2.6: Representative experimental set-up with an evolving EHD instability of a polymer film under a laterally homogeneous electric field and the corresponding optical micrographs of the EHD pattern formation. (A). Thin polymer film $(h \approx 100 \mathrm{~nm})$ deposited on a bottom electrode is opposed by a second planar electrode at distance $d$ and liquefied at $T>T_{\mathrm{g}}$ with a voltage $U$ applied between two electrodes. Small $d$ generates high $E_{\mathrm{f}}\left(\sim 10^{8} \mathrm{~V} / \mathrm{m}\right)$. This results in the amplification of a surface undulation with a characteristic wavelength $\lambda(B)$, which leads to the formation of hexagonally ordered columns $(\mathrm{C})$. The origin of the destabilizing electrostatic pressure is a result of the electric field which causes the energetically unfavourable build-up of displacement charges at the dielectric interface (A), and the alignment of the dielectric interface parallel to the electric field lines $(\mathrm{C})$ lowers the electrostatic energy.

\subsubsection{EHD Lithography Induced by a Laterally Varying Elec- tric Field}

The intrinsic length scale of EHD instabilities in thin liquid films is on the order of micrometers. It is possible to decrease the length scale to technologically interesting feature sizes by imposing a laterally heterogeneous field variations smaller than the intrinsic wavelength. This can be accomplished by using a topographically structured upper electrode. For an applied electric field, the topographic pattern is an equipotential surface. The variation in the inter-electrode spacing $d$ therefore results in a lateral inhomogeneous field.

When a laterally varying electric field is applied to the capacitor device, the instability is focused in the direction of the highest electric field (Fig. 2.7B). Since, $p_{\text {el }} \propto 1 / d^{2}$ (Eq. 2.31) the electrostatic pressure is much stronger for smaller electrode distances, pattern replication of topographically structured upper electrode proceeds at a shorter time scale than the regular EHD structure formation. The instability is guided towards the protruding patterns of the top mask, and the liquid polymer is drawn towards these protrusions, forming positive replica (Fig. 2.7C). A successful pattern replication requires harmonizing the length scale of the master pattern and of the instability characteristic wavelength. 
This EHD lithographic technique harnesses the initial film instability to replicate a wide range of patterns with high-fidelity and nanometric lateral length scales.

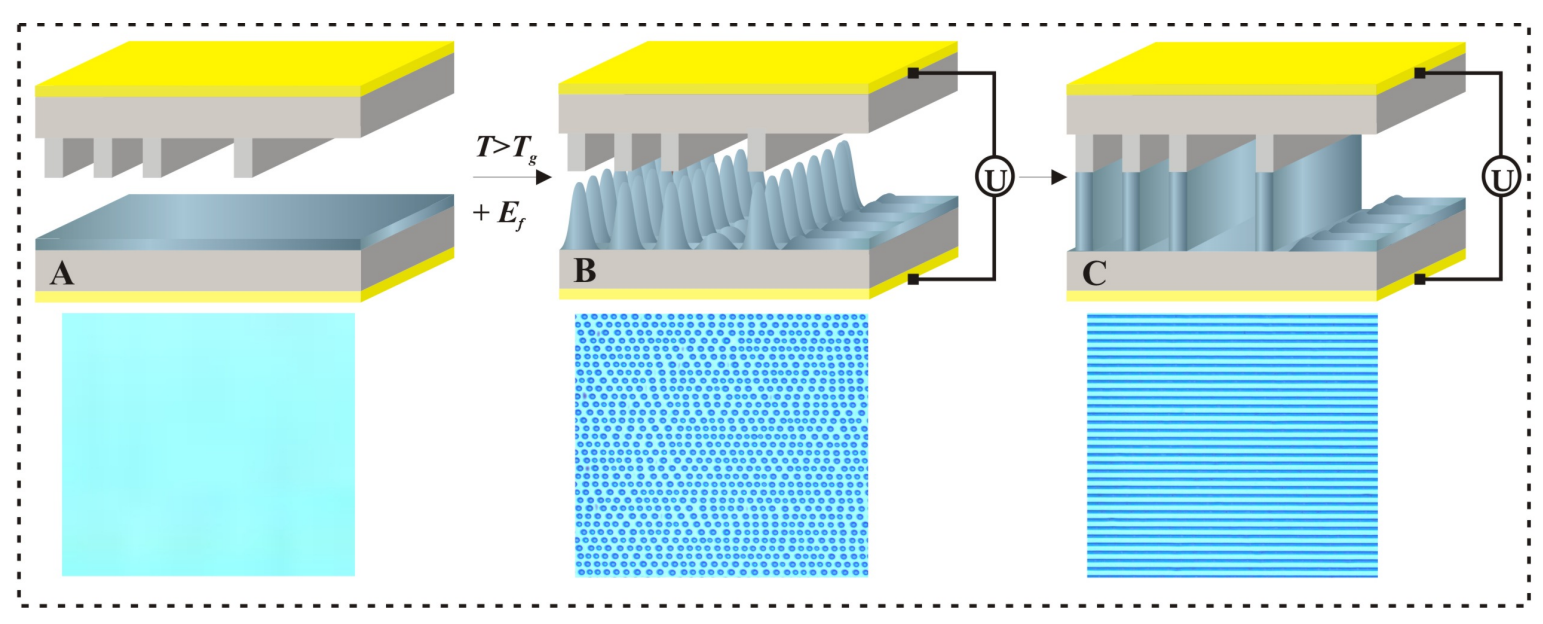

Figure 2.7: Top: Schematic of experimental device during the EHD lithography process under a laterally heterogeneous electric field. (A). A structured upper plate creates a heterogeneous force field focusing the instability towards the protruding structures (B). In (C) a positive replica of the master pattern is transferred into the polymer. In unstructured regions, the film remains stable on a much longer timer scale. Bottom: Optical micrographs of the pattern replication of a line grating, imaged after immobilization of the thin film and removal of the master electrode: (A). Initial capillary plugs spanning the substrate and protruding lines; (B) increasing coalescence of the capillary plugs; and (C) final replicated grating. 


\section{Experimental Tools and Analytical}

\section{Techniques}

This chapter describes the principles of the EHD patterning approach including the relevant sample preparation and characterization techniques. The materials used along with specific experimental details vary for each given study (i.e., for each one of the following chapters) and are therefore included in the relevant chapters.

\subsection{Overview of the Experimental Procedure}

The experimental set-up is standard for all experiments. A representative capacitor-like assembly (Fig. 3.1) consists of a bottom electrode covered by a thin film, a small air gap and a top electrode. Overview of the generic experimental procedure is summarized below.
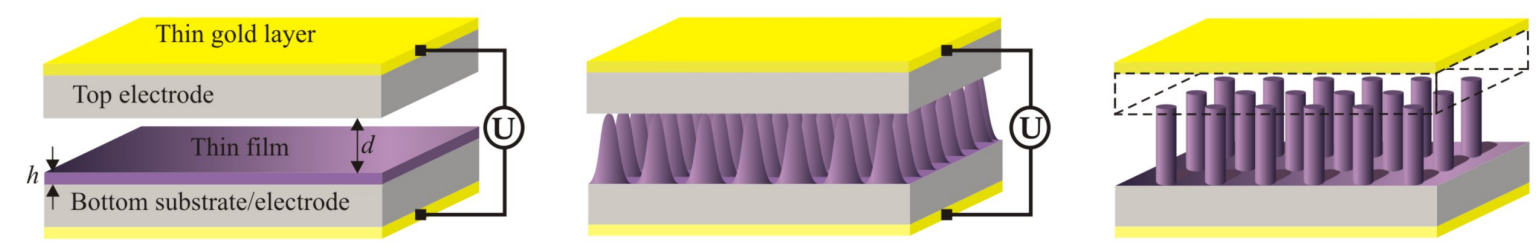

Figure 3.1: Schematic representation of the experimental set-up. Left hand side: A thin polymer film with initial film thickness $h$ is assembled between two parallel electrodes that are spaced apart by a distance $d$. With a voltage $U$ applied, the polymer film destabilizes, and an instability forms throughout the film with a characteristic wavelength (shown in the middle). With increasing amplitude of the film thickness variations, as visible on the right hand side, the film is pinned to the top electrode, thereby bridging the gap between the two electrodes.

Thin polymer films $(h \sim 30-1100 \mathrm{~nm})$ were deposited on a solid substrate by spincoating from a solution with a typical polymer concentration of $2-5 \mathrm{wt} \%$. Opposing it, a second electrode was placed at a certain distance, leaving a small air gap, $d$. Top electrodes with planar as well topographically structured surfaces (created by electron beam lithography) were used. Both capacitor plates were electrically contacted and a 
voltage of $10-100 \mathrm{~V}$ was applied across the two electrodes while the temperature of the device was raised above the glass transition temperature, $T_{\mathrm{g}}$, of the polymer film for periods of time ranging from several seconds to several hours. The structure that had formed during this period was frozen-in by reducing the temperature below $T_{\mathrm{g}}$ or below the crystallization temperature, $T_{\mathrm{c}}$ of the material and removing the electric field. After disassembling the device, the film was characterized by optical microscopy (OM) and tapping mode atomic force microscopy (AFM). In certain cases additional analysis was carried out using transmission electron microscopy (TEM) and scanning electron microscopy (SEM).

\subsection{Materials}

\subsubsection{Substrates}

Variety of substrates were used for EHD patterning depending on experimental requirements. Test grade silicon $(\mathrm{Si})$ wafers ( $p$-type, crystal orientation $<100>$ ) with a resistivity of $10-50 \Omega / \mathrm{cm}^{2}$, covered by a thin $(\sim 2 \mathrm{~nm})$ native oxide layer (WaferNet, GmbH Germany) were used as main substrates for EHD patterning. These substrates were cut into small pieces (typically, $1 \times 1 \mathrm{~cm}^{2}$ ) and used as supporting electrodes for deposited thin films or as planar top electrodes. The wafers were highly polished on one side and had a surface roughness below $1 \mathrm{~nm}$. In addition, Si wafers that were patterned by electron-beam (e-beam) lithography were used as top electrodes for the EHL experiments (obtained from Xtreme Lithography, Ulm, Germany). Transparent indium-tin oxide (ITO) covered glass slides with a resistivity of $80 \Omega / \mathrm{cm}^{2}$ were also used as substrates, allowing the in-situ optical monitoring of the pattern formation or replication process. ITO is a conductive material that consists of indium (III) oxide and tin (IV) oxide and renders (non-conductive) glass slides conductive. ITO glass slides are important in electronic devices such as liquid crystal displays (LEDs), organic light emitting diodes (OLEDs) and in the electric field experiments described in this thesis.

\subsubsection{Patterned Materials}

A range of materials has been patterned using the EHD method. Starting with typically used polymers (e.g., polystyrene), the materials pool has been extended to low viscosity, biodegradable polymers (Chapter 4), carbon-nanotube (CNT) polymer composites (Chapter 5), combinations of these materials for multilayer patterning (Chapter 6), crystalline materials (Chapter 7), conductive polymer (Chapter 8) and block-copolymers (Chapter 9). The standard polymers included: polystyrene (PS) and poly(methylmethacrylate) (PMMA), while the low viscosity range consisted of: 
ethyl cellulose (EC), poly(vinyl alcohol) (PVA), poly(vinyl acetate) (PVAc), poly(vinyl pyrrolidone) (PVP) and polycaprolactone diol (PCL-diol). Crystalline materials that were patterned via EHD process were: poly (Dimer Acid-co-1, 6-hexanediol-co-Adipic Acid) (PDHA) and polycaprolactone (PCL). PS and PVA were doped with multiwall carbon nanotubes (MWCNTs) to create nanocomposites. The polymers and their characteristics used for patterning in this study are summarized in Table 3.1. All the materials were dissolved in the appropriate solvent for minimum 2 hours to guarantee their complete dissolution in the solvent. All the used polymers had a narrow molecular weight distribution and were used without further purification.

Chemical vapour deposition (CVD) grown MWCNTs (outer diameter: $8-15 \mathrm{~nm}$, purity: higher than 95\%) were purchased from Cheap Tubes Inc and further incorporated into the polymer matrix of PS and PVA to form nanocomposite materials. Conductive polymer, polypyrrole (PPy) soluble in organic solvents was synthesized in this study. Further details related to this experimental procedures regarding nanotubesbased polymer composite and conductive polymer exploited for patterning will be given in Chapters 5 and 8, accordingly.

Table 3.1: Characteristics of the polymers used in the experiments described in the later chapters. $M_{\mathrm{n}}$ corresponds to the number-averaged molecular weight, $\eta$ to the viscosity, $T_{\mathrm{g}}$ to the glass transition temperature, $\gamma$ to the surface tension and $\varepsilon_{\mathrm{p}}$ to the dielectric constant. Viscosity and surface tension values were obtained at temperatures of $50^{\circ} \mathrm{C}$ above the $T_{\mathrm{g}}$ of each polymer.

\begin{tabular}{cccccccc}
\hline \hline Polymer & $\begin{array}{c}M_{\mathrm{n}} \\
\mathrm{Kg} / \mathrm{mol}\end{array}$ & $\begin{array}{c}\eta \\
\mathrm{Pa} \mathrm{s}\end{array}$ & $\begin{array}{c}T_{\mathrm{g}} \\
{ }^{\circ} \mathrm{C}\end{array}$ & $\begin{array}{c}T_{\mathrm{m}} \\
{ }^{\circ} \mathrm{C}\end{array}$ & $\begin{array}{c}\gamma \\
\mathrm{mN} / \mathrm{m}\end{array}$ & $\varepsilon_{\mathrm{p}}$ & Source \\
\hline PS & 105 & $1.5 \times 10^{4}$ & 100 & 240 & 30 & 2.5 & Polymer Standards \\
PMMA & 92 & & 105 & 160 & 29.7 & 3.6 & Polymer Source Inc. \\
EC & 0.55 & 0.084 & $100-130$ & $160-190$ & 28.5 & 3.5 & Fluka \\
PVA & 0.49 & 0.073 & 85 & 230 & 50 & 2 & Sigma-Aldrich \\
PVAc & 0.77 & 0.225 & $25-30$ & 45 & 57.4 & 5.8 & Sigma-Aldrich \\
PVP & 7 & 1.032 & 110 & $150-180$ & 56 & 2.3 & Sigma-Aldrich \\
PCL-diol & 1.25 & 0.557 & 45 & $55-60$ & 49 & 2 & Sigma-Aldrich \\
PDHA & 0.9 & 0.550 & 20 & - & - & 3.3 & Sigma-Aldrich \\
PCL & 10 & 1.200 & -60 & 60 & 29.5 & - & Sigma-Aldrich \\
\hline \hline
\end{tabular}

\subsubsection{Solvents}

All the materials utilized in this study were solid at room temperature. Therefore, a range of solvents was used to dissolve the materials to allow formation of spin-coatable solutions. The predominantly used materials and their solvents and non-solvents are listed in Table 3.2. 
Table 3.2: Solvents and non-solvents for mainly used polymers.

\begin{tabular}{ccc}
\hline \hline Polymer & Solvents & Non-Solvents \\
\hline PS & Cyclohexane, cyclohexanone, MEK & $\begin{array}{c}\text { Acetone, acetic acid, alcohols } \\
\text { diethyl ether, diols }\end{array}$ \\
PMMA & Ethanol, IPA, MEK & $\mathrm{CCl}_{4}$, m-cresol, diethyl ether \\
EC & Chloroform, DCM, acetic acid & Hexane, $\mathrm{H}_{2} \mathrm{O}$, nitromethane \\
PVA & $\mathrm{H}_{2} \mathrm{O}, \mathrm{DMF}$ & Hydrocarbons, THF \\
PVAc & Esters, toluene, acetone, & Saturated hydrocarbons, ethanol, \\
& methanol, THF, aromatics, alcohols & $\mathrm{H}_{2} \mathrm{O}$, cyclohexanole \\
PVP & $\mathrm{H}_{2} \mathrm{O}$, methanol, ethanol, acetic acid & $\mathrm{CCl}_{4}$, benzene, hexane, \\
& & cyclohexane, toluene, xylene \\
\hline \hline
\end{tabular}

\subsection{Sample Preparation}

\subsubsection{Thin Metal Film Evaporation on Substrates}

A good electrical contact is necessary for the EHD patterning process. However, the thin oxide layer covering the silicon wafer often causes an unwanted large contact resistance. To avoid electrical charging effects while applying an external field a thin gold layer was evaporated onto the back side of the wafers. First, a $2 \mathrm{~nm}$ thick chromium layer was evaporated onto the unpolished backside of the substrate, in order to provide good adhesion of the metal on the $\mathrm{SiO}_{x}$ surface, followed by the deposition of $100 \mathrm{~nm}$ thick gold layer. Silver paint (Electrodag 1415M) was further used to contact the electrodes.

Evaporation was performed in an Edwards Evaporator FL 400 (40 mm chamber, $360 \mathrm{~mm}$ rotating disc). Chromium and gold were sequentially deposited in vacuum (typically, $1.5-3 \times 10^{6} \mathrm{mbar}$ ). To sublimate chromium and evaporate gold, the current through the molybdenum boat (which accommodates the material to be evaporated) was increased until the boat started glowing white, indicating the melting of the material. The current was then further increased to adjust the deposition rate. Following the shutter opening, the deposition rate was adjusted to $0.1-0.2 \mathrm{~nm} / \mathrm{s}$ and the deposition was carried out. The deposited thickness was monitored in-situ by a quartz microbalance.

\subsubsection{Cleaning of the Substrates}

To establish a reproducible and well defined substrate surface, it is essential to remove surface contaminations and organic particles by a thorough substrate cleaning proce- 
dure. Si wafers and pre-structured electrodes were first cleaned in a Piranha [127] solution, consisting of $3: 1(98 \%) \mathrm{H}_{2} \mathrm{SO}_{4}$ : $(30 \%) \mathrm{H}_{2} \mathrm{O}_{2}$ heated to $80^{\circ} \mathrm{C}$ for $30 \mathrm{~min}$, followed by rinsing with deionized water and subsequent drying under nitrogen. This process removed most of the organic matter and chemical contaminants.

To remove the ITO spikes, the substrates were scrubbed for several minutes in a hot sodium dodecyl sulphate (SDS) soap solution $\left(70^{\circ} \mathrm{C}\right)$ and rinsed with Millipore water. To remove the soap and other surface contaminations, the substrates were further cleaned in an ultrasonic bath in acetone and isopropanol and then spun dry. The substrates were then heated for 10 minutes at $120{ }^{\circ} \mathrm{C}$ in a convection oven, followed by 20 minutes of UV-ozone treatment. Immediately before film deposition and device assembly all substrates and master electrodes were subjected to jet of carbon dioxide crystals $\left(\mathrm{CO}_{2}\right.$ snow-jet $)$ [138] to remove macroscopic dust particles and organic contaminants. All substrates were used immediately after the cleaning process.

\subsubsection{Snow-Jet Cleaning}

Snow-jet cleaning process, first established in 1986, [55] is a single-step procedure used to clean the substrates in our experiments. The snow-jet apparatus consists of carbon dioxide $\left(\mathrm{CO}_{2}\right)$ source, a nozzle with an internal orifice and tube to transport the $\mathrm{CO}_{2}$ from the source to the nozzle. The expansion of high pressure $\mathrm{CO}_{2}$ is guided through a nozzle to output a stream of small $\mathrm{CO}_{2}$ ice crystals which is aimed at a solid surface.

Snow-jet cleaning process is based on combination of momentum transfer by the impacting dry crystals and the solvent properties of liquid $\mathrm{CO}_{2}$. The aerodynamic drag exerted by a gas stream flowing over surface is insufficient to remove the adhered particles. However, the pressure and the temperature drop from the expanding high velocity of $\mathrm{CO}_{2}$ gas stream nucleate the formation of dry ice crystals, which upon impact provide sufficient momentum to remove sub-micron and micron sized particles that have adhered to the surface (Fig. 3.2). In addition, the dry ice liquefies upon impact, dissolving organic residues. Upon rebound and solidification of $\mathrm{CO}_{2}$, the high-velocity stream carries away the newly formed particles containing the chemical heterogeneities with efficiencies up to $99.99 \%$.

\subsubsection{Surface Energy Modification}

All the experiments were performed in capacitor geometry. Polymer structure spanning the two electrodes had tendency to adhere to both substrates. Following the mechanical removal of the top electrode, the structure either adhered to the top or bottom substrate or was ripped apart. To facilitate a non-destructive release and to preserve the formed patterns intact during dismantling of the set-up, the surface energy of the top plate 


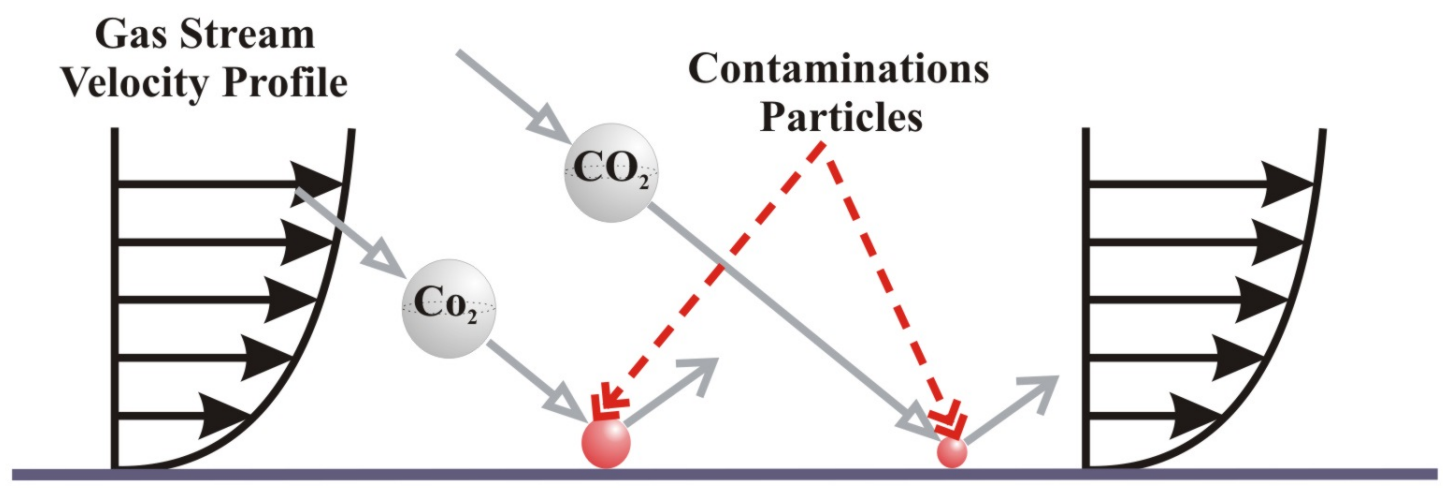

Figure 3.2: Schematic representation of the snow-jet process: the aerodynamic drag forces exerted by the gas flowing over the surface overcome the adhesion force of adsorbed particles removing the contaminations. The $\mathrm{CO}_{2}$ reaches its liquid state upon impact. The pressure at the interface of $\mathrm{CO}_{2}$ crystals exceeds the triple point pressure of a dry ice particle resulting in its liquefaction. Liquid $\mathrm{CO}_{2}$ dissolves non-polar and hydrocarbons substances, and upon bouncing off the surface the particle re-solidifies while carrying the contaminations away in the gas steam.

was reduced by depositing a self-assembled monolayer (SAM) of alkanes. A SAM can be prepared by a simple immersion of a substrate in a solution containing alkane chains such as octadecyltrichlorosilane (OTS) with actively binding end-groups. OTS forms covalent bonds with the oxygen groups of a silicon oxide surface and self-assembles into a highly-ordered monolayer (Fig. 3.3). This chemical reaction of silanization [138, 139] renders the oxidized Si surface appolar (low surface energy), and thus, provides a route to reduce adhesion of the patterned material to the silicon surface.

For our purpose, a non-stick $\mathrm{SAM}$ on $\mathrm{SiO}_{x}$ was deposited from liquid OTS phase. In order to remove all organic contaminations and hydroxylate the surface, $\mathrm{SiO}_{x}$ covered substrates were first cleaned using snow-jet followed by immersion in Piranha solution rinsing with Millipore water and drying in a stream of a dry nitrogen gas. Silanization was performed by immersion of the substrate in the freshly prepared silane solution ( $0.25 \%$ OTS in hexadecane). Physisorption of OTS monolayer on the substrate surface proceeds via silanes binding to hydroxyl-terminated $\mathrm{SiO}_{x}$ layer. After drying in dry nitrogen gas, water contact angles, typically above $90^{\circ} \mathrm{C}$ were obtained, sufficiently high for our experiments.

\subsubsection{Thin Film Deposition: Spin-Coating Process}

Typically a solution of the relevant material or composite was prepared at concentrations of $2-5 \mathrm{wt} \%$, followed by filtering through a Teflon membrane with a pore diameter of $100 \mathrm{~nm}$. Subsequently, thin films with thicknesses on the order of $100 \mathrm{~nm}$ were pre- 

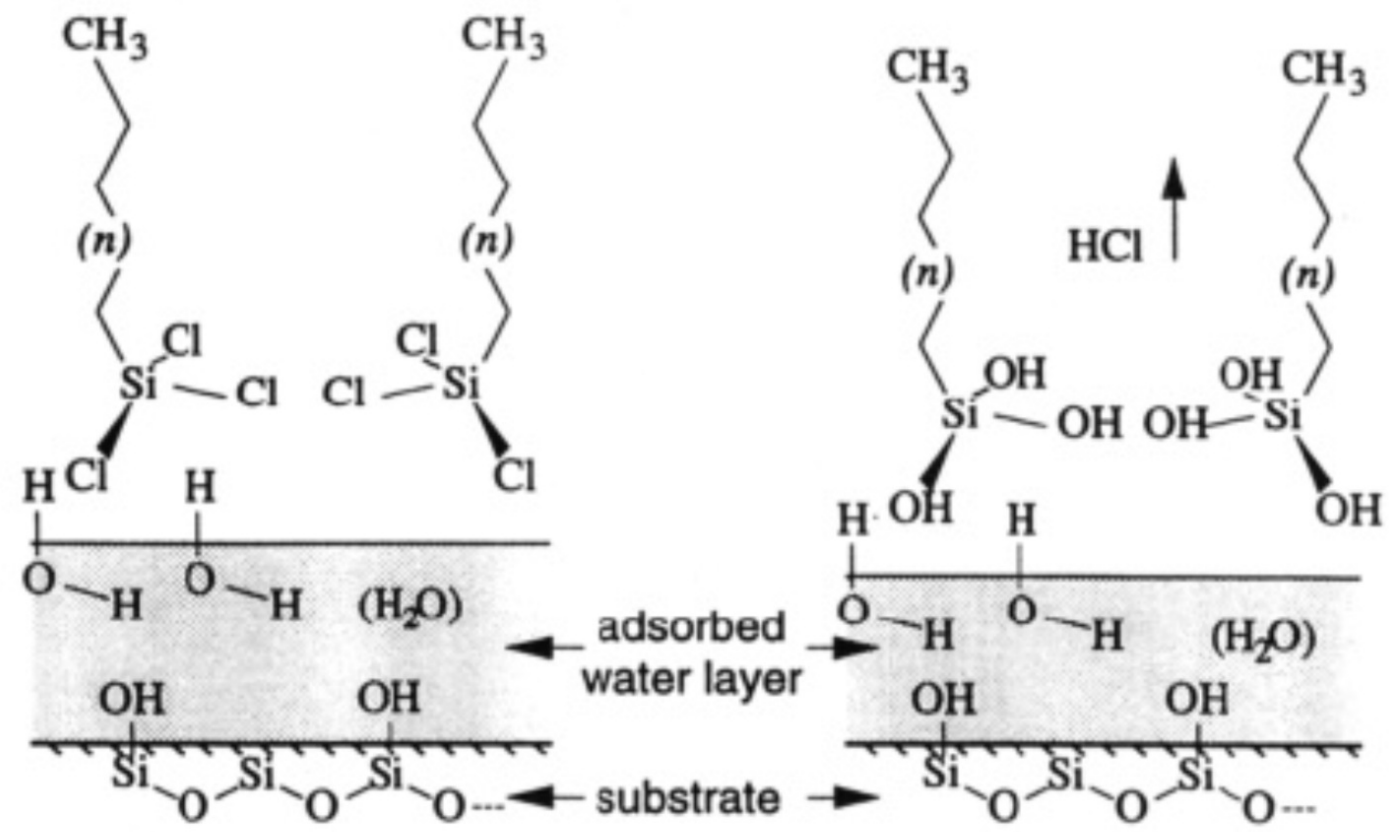

1) Physisorption

\section{2) Hydrolysis}

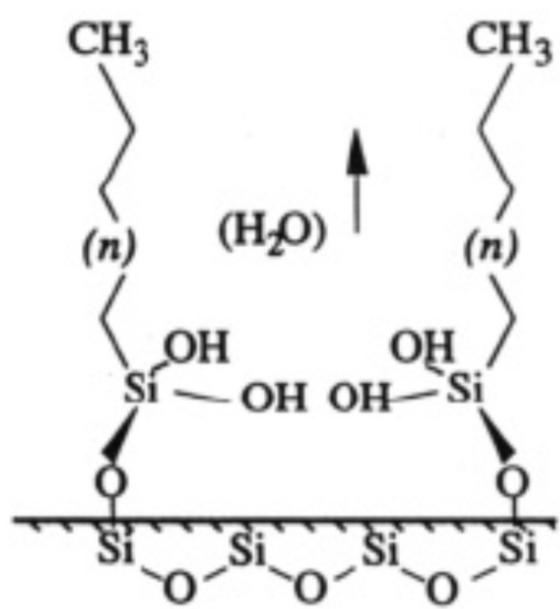

\section{3) Covalent grafting to the substrate}

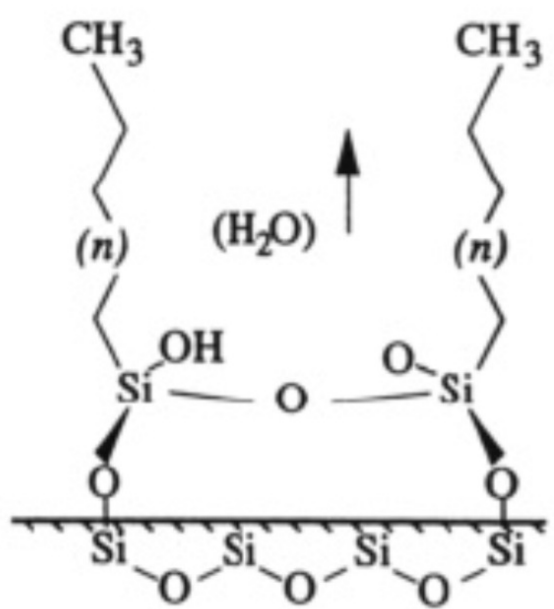

\section{4) In-plane reticulation}

Figure 3.3: General scheme for the formation of a self-assembled monolayer (reproduced from ref [19]): hydrated Si wafers with a native SiOx layer allow the physisorption of organic molecules (stage 1). The trichlorosilane groups hydrolise and form trisilanols (stage 2). A lateral mobility of the chain silanes on $\mathrm{Si}$ is promoted by an adsorbed water layer yields in-plane reorganization of the silane moecules. The correct thermodynamic conditions allow the formation of a densely packed monolayer comprised of vertical chains. Long silanization times lead to grafting of the monolayer to the surface via the formation of covalent siloxane bonds at the head group-substrate interface (stage 3 ). This is followed by intermolecular cross-linking of the trisilanol groups (stage 4). 
pared via spin-coating an adequate amount of solution onto clean substrate used as an electrode. To yield highly homogeneous films and to minimize contaminations, the spin coating procedure was performed in a clean room conditions. Spin-coating is a common process used in the semiconductor industry to uniformly coat substrates with a thin polymer layer. This is accomplished by wetting the substrate (held by on the spin a vacuum chuck) with certain amount of solution and subsequently rotating it at a high speed, $100-1000$ rotations per minute (rpm) (Fig. 3.4). Centrifugal forces spread the solution to a homogeneous film, with increasing angular spinning speeds resulting in thinner films. A four step process can be used as a model description for the spin coating process:

1. Deposition of polymer solution onto the substrate. Excess of coating solution may be applied compared to the required amount.

2. The substrate is accelerating towards the final rotating speed. Most of the excess solution is centrifuged off the substrate, leaving a thin layer.

3. The substrate is spinning at constant rotation rate. The viscous forces dominate the gradual and homogeneous thinning of the film at this stage.

4. Evaporation, which is dominant in this stage leads to a loss of solvent and an increase in the viscosity (and density) of the polymer solution, slowing the shear thinning of the film. Finally, the sufficient loss of the volatile solvent leaves behind thin homogeneous film.

The competition between the centrifugal force and the evaporation rate of the solvent determines the thickness of the dry film. The thickness of the resulting film is controlled by the initial solution concentration, its viscosity and the rotation speed. For well chosen parameters, spin-coating results in homogeneously thin polymer films.

The thin film deposition process was performed using Laurell (VWS-400B-6NPPLTE) spin coater. The humidity in the chamber was controlled via a constant dry $\mathrm{N}_{2}$ gas flow and the desired film thicknesses were achieved after calibration of the spinning time, speed and acceleration.

\subsection{Characterization Techniques}

A major part of the experimental analysis relies on imaging techniques. While optical microscopy (OM) essentially yields micrographs of the generated patterns, atomic force microscopy (AFM) and scanning electron microscopy (SEM) provide more detailed information regarding structures' lateral and vertical dimensions along with an improved 


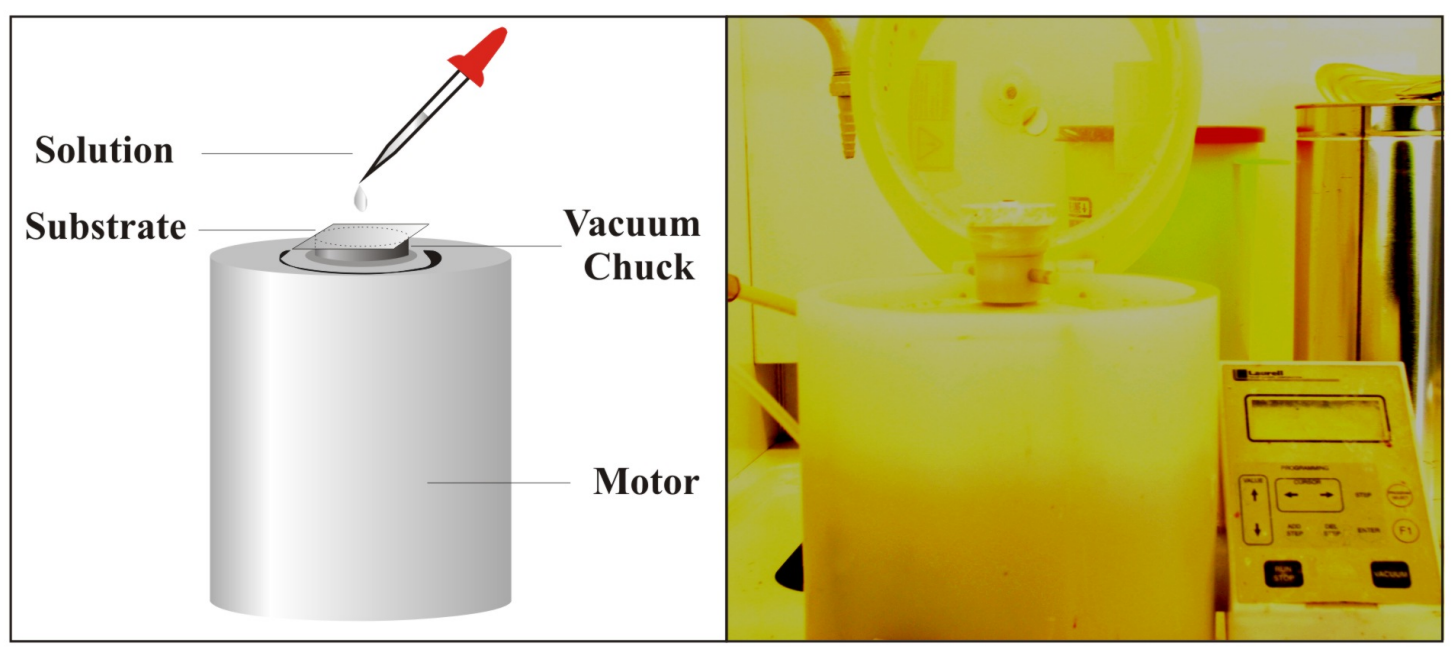

Figure 3.4: Schematics and image of the spin-coater.

lateral resolution. Analysis of cross-sectional transmission electron microscopy (TEM) images provides additional insights into the formed or replicated patterns and substructures on the molecular level.

\subsubsection{Optical Microscopy}

Spectacular interference colours arise from thin films on top of silicon wafers, when a refractive index of the substrate is sufficiently different from that of the covering material. Thickness variations even on the order of 10 nanometers lead to a color change that can be observed by optical microscopy. A high time resolution which enables in situ experiments at variable magnifications and the characterization of lateral topographic features ranging from $5 \mathrm{~mm}$ to $200 \mathrm{~nm}$, are the reasons for the wide application of $\mathrm{OM}$ in research. [47, 116]

Colors in the polymer film are the result of the interference of the two reflected beams at the polymer-air and at the polymer-substrate interfaces of the incident white light of the microscope. These two beams can either interfere constructively or destructively. Interference from a film with given thickness therefore enhances or cancels particular wavelengths, and the film will appear colored. The colors follow a periodic rainbow sequence with increasing film thickness, as illustrated for a Polystyrene (PS) film on a Si wafer (Fig. 3.5). All optical microscopy instruments are fundamentally limited by the resolution they can achieve. The resolution is defined as the minimum separation between two points that can be resolved (i.e., two distinct entities can be visualized). In a perfect optical system, the maximal resolution is limited by the nu- 
merical aperture of the optical components and by the wavelength of the light.

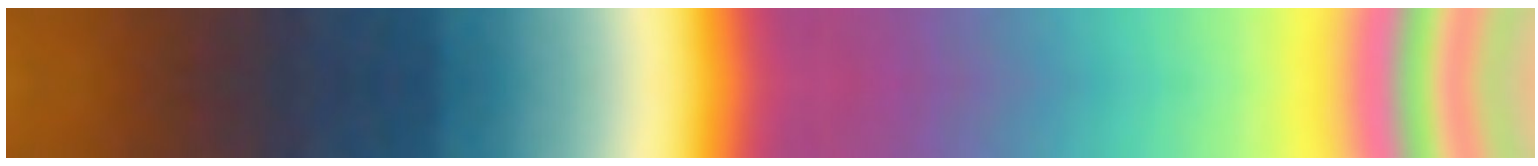

Figure 3.5: Interference colours from a thickness gradient in a PS film on a silicon wafer, with refractive indices of 1.6 and 4.1, respectively. (Reproduced from [127]). Starting from brown $(20 \mathrm{~nm})$ on the left, through light purple $(400 \mathrm{~nm})$, pink $(520 \mathrm{~nm})$ and eventually to alternating light green and pink $(\sim 1.5 \mu \mathrm{m})$, the color eventually changes to a transparent grey for very thick films.

To qualitatively analyze pattern formation in thin polymer films, a bright-field reflection microscopy (Olympus Optical Microscopes GX61 and BX40) were employed in our experiments. The reflection of white light from the sample enabled us to resolve submicrometer features. The post-facto acquired data in our experiments is complemented by the in-situ monitoring of EHD pattern formation and replication in thin films, using a digital camera and imaging software (Carl Zeiss VisioCam).

The comparison of the observed with Fig. 3.5 is a straightforward way to directly observe submicron features and small thickness variations in thin polymer films.

\subsubsection{Atomic Force Microscopy}

The Nobel Prize for Physics in 1986 was awarded to the inventors of the scanning tunneling microscope (STM), Gerd Binnig and Heinrich Rohrer. Since then, this high resolution imaging tool has developed rapidly, evolving into family of related techniques which are classified in the general category of Scanning Probe Microscopy (SPM) techniques. Of these techniques, the atomic force microscope (AFM) is today widely used to obtain precise information about both surface topography and surface mechanical properties.

The concept of AFM is based on a sharp tip made from silicon or silicon nitride attached to a flexible cantilever with a spring constant (of $1-100 \mathrm{~N} / \mathrm{m}$ )(Fig. 3.6C). Upon approach to a surface, this tip experiences attractive and repulsive interactions which are measured by the bending of the cantilever. By adjusting the vertical displacement of the tip in such a way that the cantilever bending is constant, when scanning the tip laterally across the surface, the surface topography is reconstructed. Alternatively, a force-distance curve can be acquired at a fixed lateral position, thereby measuring the forces acting on the probe.

The two most commonly used modes of operation are contact mode AFM and tapping mode AFM, which are conducted in air or liquid environments. During a line-by-line scan across the surface in the scanning mode, the tip follows sample's 
topography while experiencing a constant force and maintaining the distance between the sample and tip relatively constant. The tip-sample interaction is monitored by deflecting a laser beam off the back of the cantilever onto a split, position sensitive photodiode detector. The position of the laser reflection spot on the diode provides the input in a feedback loop which is used to maintain a constant tip-sample distance. This is accomplished by adjusting the $z$-position of the tip by altering the voltage applied to a piezoelectric ceramic onto which the cantilever (or the sample) is mounted.

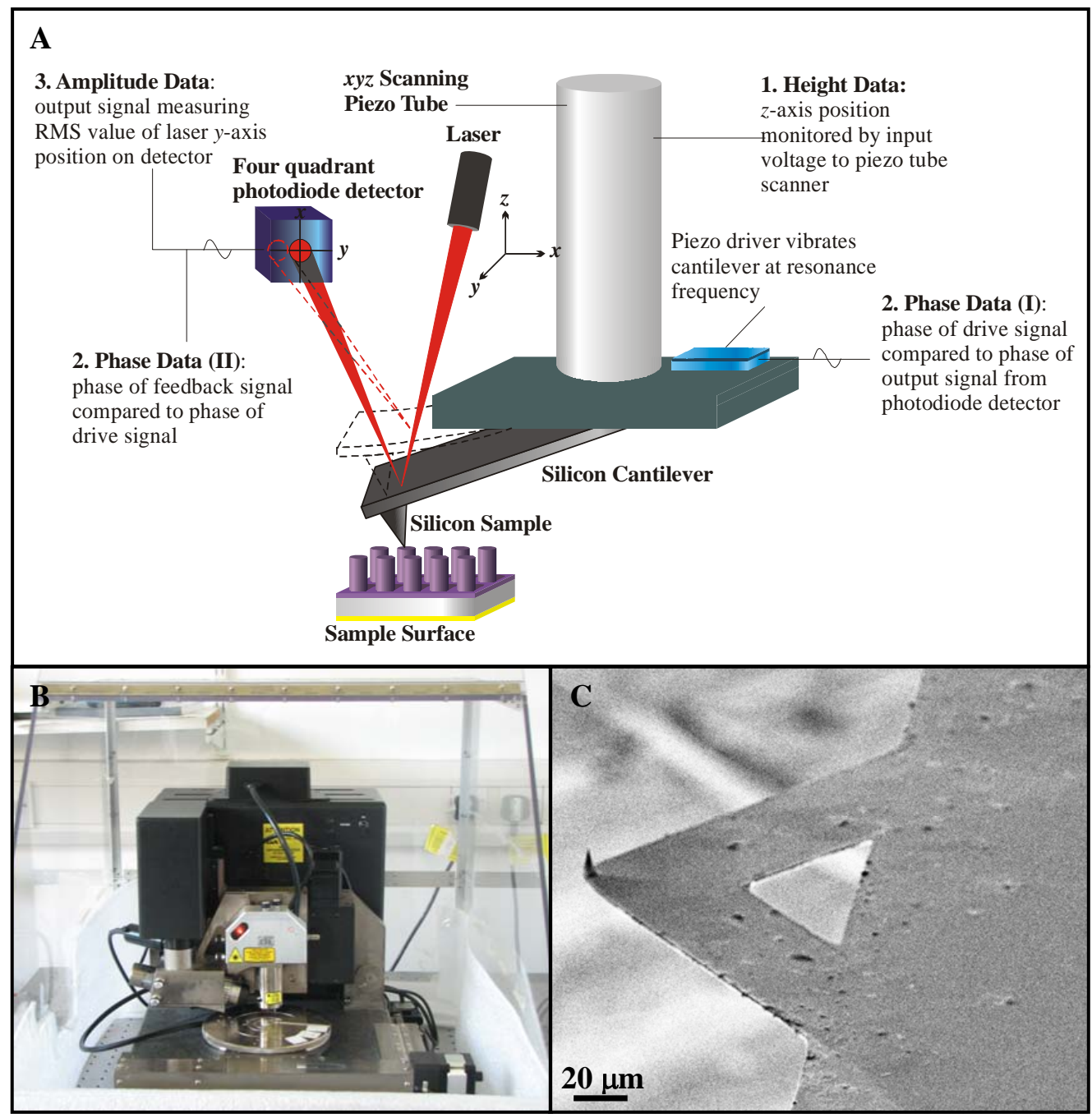

Figure 3.6: (A). Three types of data collected in tapping mode AFM: Height Data: The vertical position of the probe tip is monitored by noting changes in the $z$-displacement of the $x-y-z$ scanning piezo tube. Phase Data: Monitoring of the change in phase offset, or phase angle, of the oscillating cantilever with respect to the drive signal. Amplitude Data: The amplitude of the cantilever is monitored by the photo diode detector. RMS value of the laser signal on the split photodiode. (B). Image of the AFM microscope used in this study. (C). Scanning electron microscopy image of a typical AFM probe used in this study. 
The applied voltage that is required to maintain the tip to sample distance constant is a measure for the piezo displacement, thereby reconstructing the topography as the tip is scanned across the surface.

In tapping mode, probing is performed via an intermittent contact of the tip with the sample, thereby minimizing the lateral shear force as the tip is scanned across the surface. This mode is particularly useful to prevent damage in soft, fragile or adhesive surfaces during scanning. Here, the cantilever is driven slightly below its resonance frequency $(200-300 \mathrm{KHz})$ at a constant set-point oscillation amplitude on the order of $10 \mathrm{~nm}$ while probing the sample by lightly "tapping" the tip on the surface. The laser deflection method is used to detect the root-mean-square (RMS) amplitude of cantilever oscillation. A feedback loop maintains a constant oscillation amplitude by moving the scanner vertically at every $(x, y)$ data point. Recording these movements forms the topographical image (Fig. 3.6A).

AFM measurements were performed using a Nanoscope IV Dimension 3100 (Veeco Instruments Inc.) microscope operated in the tapping mode (Fig. 3.6B). Image processing and analysis was carried out with the instrument software version V612r2 and V530r2. AFM measurements were used to acquire important experimental parameters including the initial film thickness, the lateral distances between the generated structures, their heights and diameters (i.e., their aspect ratio).

\subsubsection{Scanning Electron Microscopy}

The scanning electron microscope (SEM) images a sample by using high-energy electrons beam to generate variety of signals arising from the surface of specimens. These signals are derived from interactions of the electrons with atoms at the surface of the sample, and they provide information regarding the morphology, chemical composition, topography and crystallographic structure. A meaningful lateral resolution is achieved as a result of the narrow focus of the electrons and a large depth of field producing good three-dimensional image of the sample.

An electron gun (either thermionic or field emission) is used as source for the electron beam. The electrons are guided and accelerated to the sample by a series of electromagnetic lenses in the electron column. This confined electrons beam is focused by condenser lenses to a very fine, monochromatic beam spot on the order of a few nanometres, which is scanned over a rectangular area of the sample surface by electromagnetic deflection coils. The resolution and depth of field of the image are determined by the beam current and the spot size on the sample, which are adjusted with one or more condenser lenses and the probe-forming objective lenses. Repeated random scattering and adsorption within the volume of the specimen during the primary electron beam interactions result in an energy loss of the high-energy electrons and in their re- 
flection by elastic scattering, in emission of secondary electrons by inelastic scattering, and, in the emission of electromagnetic radiation, each of which can be detected by specialized detectors.

Secondary electrons are the most common signal used for investigations of surface topography and morphology. They are produced as a result of interactions between the electron beam and weakly bound electrons in the conduction band of the specimen atoms, which receive sufficient kinetic energy to be ejected from the atom. All electrons emitted from the specimen with energy less than $50 \mathrm{eV}$ are considered as secondary electrons. The emitted electrons are collected by detectors, the signal is amplified and displayed on a cathode ray tube (CRT) or television screen. In addition, high energy backscattered electrons are valuable for illustrating contrasts in composition in multiphase samples.

Samples for SEM imaging were prepared by placing a post-experiment, disassembled substrate with the generated patterns on an inclined or cross-sectional holder, allowing the visualization of the top and cross-sectional view. Scanning electron micrographs were obtained using a thermally assisted Field Emission Scanning Electron Microscope (FESEM, LEO VP 1530) with a lateral resolution of $2-5 \mathrm{~nm}$.

\subsubsection{Transmission Electron Microscopy}

Max Knoll and Ernst Ruska were the pioneers developing and building the first microscope in 1931 with a greater resolution than that of light, the nowadays well known transmission electron microscope (TEM). TEM is a microscopy technique that uses a beam of electrons, rather that light, to image samples in transmission.

The components of a TEM are shown in Fig. 3.7: the vacuum system, the electron emission gun (generating an electron beam), electrostatic plates (guiding and manipulating the beam) and a series of electromagnetic lenses (condenser, objective and projector lenses). In the TEM, electromagnetic lenses are used to focus and direct the electron beam which is transmitted through a thin specimen. The electron beam is generated using an electron gun which consists of tungsten or a lanthanum hexaboride filament which is connected to a high voltage source (i.e., $100-300 \mathrm{KV}$ ). Upon sufficient current supply, the emission source extracts electrons into the vacuum. The TEM usually operates at $10^{-6}$ Torr. The resulting electron beam is focused by multiple electromagnetic (condenser and objective) lens systems and annular metallic apertures, which are also designed to eliminate stray electrons. After transmission through the sample, focusing and magnification (which is a ratio of the distance between the sample and the objective lens' image plane) an image is formed, and projected onto a fluorescent screen. The resulting image may be recorded on photographic film or by a CCD camera linked to a computer. 


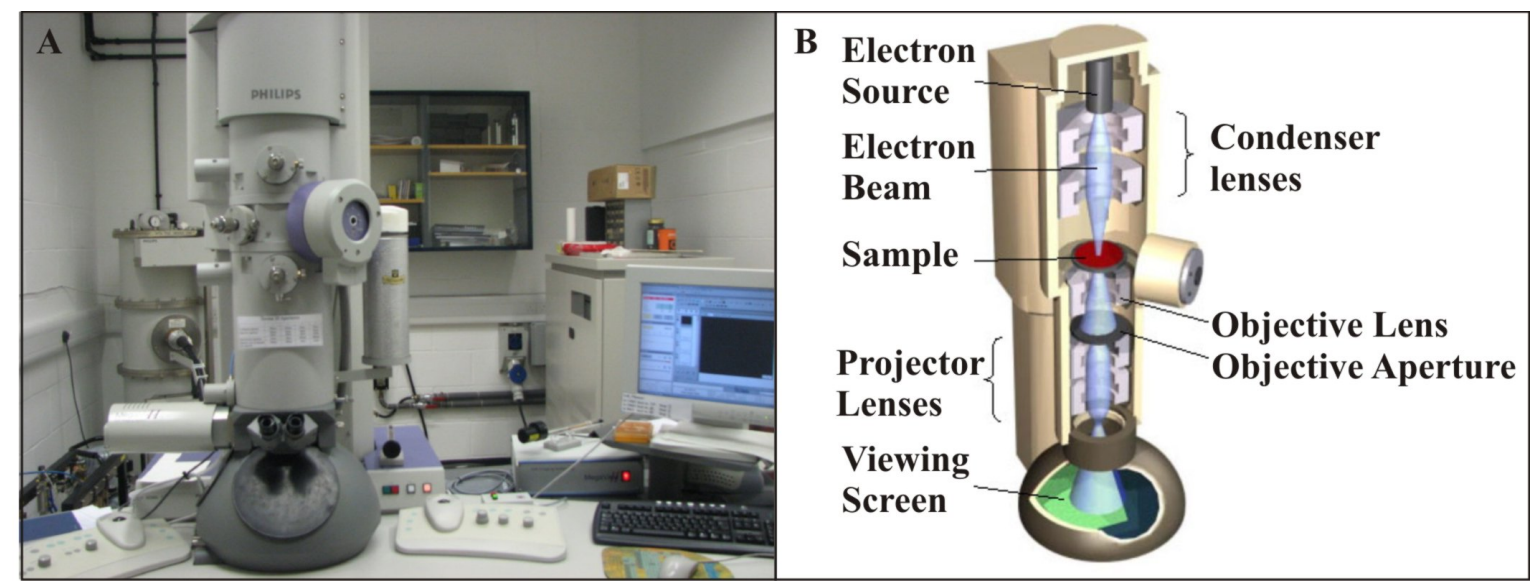

Figure 3.7: A). Photograph of FEI Phillips Technai 20 electron microscope used in this study. (B) Schematic representation of the typical internal structure of the microscope shown in(A).

The TEM contrast consists of the amplitude contrast caused by elastically scattered electrons and phase contrast originating from differences in phase of electron waves scattered through a thin specimen. Diffraction contrast arises from the elastic scatter of electrons from planes of atoms in crystalline materials.

In general, the electron waves leaving the specimen and forming an image contain the information essential for different imaging methods in TEM. Contrast formation in TEM depends strongly on the mode of the operation. Common imaging techniques are:

- Bright field imaging is the most common mode for TEM. This imaging mode selects the direct beam to form the image. In transmission, this method relies on the absorption of electrons to produce the contrast. Whilst regions with a higher atomic number or larger thickness will appear dark, the regions with low electron absorption will appear bright.

- High-resolution transmission electron microscopy (HRTEM) requires thinner specimens and higher energies of incident electron beam. Therefore, the sample no longer adsorbs electrons, but rather, acts as a complex interference grating that does not change the amplitude of beam and only modifies its phase. HRTEM is often used to investigate the crystal structure of materials.

- Electron energy loss spectroscopy (EELS) allows the selection of particular energy values which represent the interaction of the electrons with the specimen. After passing through the sample the beam exhibits different electron energies for different elements. EELS technique utilizes magnetic deflection to reject electrons based on their voltage, and due to the constant charge, thereby, their energy. 
EELS spectrometers can be operated in both imaging and spectroscopic modes, allowing for rejection or isolation of elastically scattered beam.

- Dark field (DF) imaging mode selects Bragg reflections by the placement of objective apertures in the back-focal plane of the lens rather than imaging plane which do not include the un-scattered beam. This results in a dark image wherever no sample scattering is present and only regions of the specimen which causing the electrons to scatter are projected onto the imaging system. This mode is often used to study lattice defects in crystalline materials.

- Selected area diffraction (SAD) selects a specific area of the specimen to contribute to diffraction pattern (DP) and thereby reduces the intensity of the direct beam in DP on the screen. While, in the case of a polycrystalline or amorphous material a series of concentric rings are observed, the image for a single crystal consists of an array of dots. This diffraction pattern provides information regarding the space group symmetries in the crystal and the orientation of crystal relative to the beam path.

In many cases, the studied specimens have to be sliced to extremely thin sections to ensure electron transmission. The preparation of specimens is therefore often time consuming and requires expert handling. Nevertheless, TEM analysis provides the invaluable imaging of finest details - even as small as a single column of atoms and is therefore an analytical technique with a wide range of applications ranging from cancer research, though materials science to semiconductor technology.

For TEM cross sectional imaging, a thin layer of Pt was sputtered onto the patterned films, followed by embedding into a Spurr epoxy resin. The substrate was removed and the films were sectioned using diamond knife in a Leica Ultracut Microtome, yielding sections with a thickness of $50 \mathrm{~nm}$. Reference samples were directly prepared by spin coating or directly depositing a thin transparent layer of a studied material in a liquid state on a TEM grid (typically, carbon film on $\mathrm{Cu}$ with a mesh size of $200-300 \mathrm{~nm}$ ). The specimens were analyzed using a FEI Philips Technai 20 TEM at acceleration voltages of 80,120 and $200 \mathrm{kV}$.

\subsubsection{Surface Enhanced Raman Scattering}

Raman scattering is dubbed after Sir Venkata Chandrasekhra Raman, who discovered and demonstrated the inelastic scattering of light from molecules in 1928. [114, 133] Since then, Raman spectroscopy is increasingly implemented in the physical and chemical sciences. This vibrational spectroscopic technique is complementary to other IR techniques, and is based on the exchange of the energy of vibrational or rotational 
states of molecules with light, resulting in scattering of light with an altered wavelength. Rotational and vibrational Raman spectra (and their combination) can be recorded upon illuminating the sample with a monochromatic light. A red-shifted Stokes Raman spectrum is produced upon the inelastic scattering of the incident light with molecules in their rotational (or vibrational) ground state. Since at finite temperatures some molecules are also in excited states, a blue-shifted the anti-Stokes Raman spectrum forms when they interact with the incident light. The relative intensity of the Raman lines in both spectra can be used to determine the rotational or vibrational temperature.

Nearly five decades later, it was discovered that Raman scattering signals can be dramatically enhanced (by up to $10^{6}$ fold) for molecules at structured noble metal based surfaces. [37] This surface enhanced Raman scattering (SERS) has enabled countless possibilities in sensitive analysis and diagnostics. The exact mechanism of the SERS effect is still debated in the literature. The current consensus regarding the underlying physics of this enhancement is based on the combination of an increased Raman cross-section of the molecules in contact with a metal submicrometer structure (chemical or electronic enhancement) and the electromagnetic field augmentation via localized optical fields of the metallic structures comprising the surface. These effects lead to several important properties of SERS: first, the enhancement scales as the fourth power of the local field near a metallic structure, and second, the enhancement is maximized when the incident light as well as the Raman scattered wave are in resonance with the surface plasmons of the metallic structure. SERS depends strongly on the surface on which the experiment is carried out. The shape and the aspect ratio of the metallic structures (or nanoparticles) on the surface control the enhancement factor. While maximum enhancements values for isolated single nanoparticles are $10^{7}$, considerably stronger enhancements can be achieved by utilizing organized structured surfaces. Several different materials can be used for SERS. While silver is the most common, it strongly suffers from oxidation in air. On the other hand, gold-based structures provide large enhancements, chemical robustness and biocompatibility along with straightforward functionalization chemistry, and are therefore widely used for a range of SERS applications.

SERS measurements were carried out with an InVia Renishaw Raman Microscope System equipped with $633 \mathrm{~nm}$ and $785 \mathrm{~nm}$ lasers. The spectra were typically acquired with a $10 \mathrm{~s}$ exposure time and a laser power of $3 \mathrm{~mW}$ and $10 \mathrm{~mW}$ at the sample at $633 \mathrm{~nm}$ and $785 \mathrm{~nm}$, respectively. SERS maps were acquired in Streamline mode (line scan) with $20 \mathrm{~s}$ exposure time and $6 \mathrm{~mW}$ and $100 \mathrm{~mW}$ power at $633 \mathrm{~nm}$ and $785 \mathrm{~nm}$, respectively. A $50 \times$ objective with a numerical aperture of 0.75 was used for all measurements. Optical measurements were carried out with an Olympus BX51 microscope 
equipped with an incoherent white light (halogen) source and an optical fibre coupled to a QE65000 Ocean Optics spectrometer. The spectra were calibrated with respect to those recorded on flat gold. 


\section{Rapid Patterning of Low-Viscosity Resists Using Electrohydrodynamic Lithography}

Lithography is a constantly developing field, that is essential for many advanced technologies. Establishing the ability to pattern materials with high fidelity and resolution without compromising their properties is therefore important. The rapid pace of technological advancements triggers continual developments of cost effective lithographic schemes which eventually will enable the fabrication of a broad spectrum of structures in a controlled manner within extremely short periods of time.

A novel way to induce and control the structure formation process in thin polymer films is the use of strong electric fields. Electrohydrodynamic lithography (EHL) $[130,131,175]$ uses the electric forces in a capacitor device to destabilize thin liquid films. The pressure created by electric fields at the film surface overcomes the surface tension, inducing an instability which results in a pattern with a characteristic local hexagonal order. When a laterally varying electric field is applied, the instability is focused in the direction of the highest electric field. This results in the replication of a topographically structured electrode. While the origin of the underlying EHD surface instabilities is long known, [41, 149, 159] its use for high performance lithography is relatively recent, providing a way to replicate $100 \mathrm{~nm}$-sized patterns in a simple an straight forward fashion. The benefit of EHL pattern replication is the possibility of contact-less lithography [52] along with the electric field coupling to smaller selfassembled morphologies within the film, [167] providing a way for hierarchical structure control spanning many length scales. EHL therefore enables the generation of a broad spectrum of high-fidelity patterns in a straightforward and low-cost fashion.

In this study we aim to overcome an intrinsic problem of the EHD patterning process, the rather long pattern generation times. A number of low-viscosity biodegradable materials are explored for their suitability as high-speed resists in the EHD lithography process. The use of low-viscosity glass forming polymers significantly reduces the patterning time of EHL to a few seconds compared to earlier approaches, without compromising the high fidelity of the replicated structures. An essential requirement for 
short time EHL patterning is the precise termination of the pattern formation process. To this end, a method for the on-line surveillance of the EHL process is introduced monitoring the leakage current across the device to detect the completion of EHD pattern formation. The results of this study have a technological appeal, not only because of the speed-up of the lithographic process, but also by providing an extension to additional materials that can be patterned by EHD, demonstrating the versatility of this method.

\subsection{Introduction}

EHD lithography allows the generation and replication of structures with feature sizes on sub-micron and possibly sub-100 nm lateral length scales. As a generic principle, this technique makes use of instabilities in thin liquid films confined in a capacitor device. While thin films are intrinsically stable, the application of a force at a film boundary results in the break-up of the film. Such instabilities typically exhibit one well-defined lateral length scale and a well defined dynamics. The introduction of the lateral field heterogeneities influences the initial pattern formation by directing the instability to replicate a master pattern with high precision.

One of the limiting factor has, however, been the slow replication dynamics. Typical polymeric resists require many minutes or hours for the pattern replication to complete, as shown for example by Leach and Russell. [78] This problem is intrinsic to EHL, caused by the interplay of two factors.

1. Most resists consist of high molecular weight polymers that have a high melt viscosity in their accessible temperature range. Since the electric field strength is limited by the dielectric strength of the materials in the narrow capacitor gap in Fig. 4.1, the obtainable strain rates are generally low. Reducing the molecular weight of the polymers often reduces the polymer glass transition temperature precluding the requirement of a solidification above room temperature. Consequently, glass-forming materials are required, which form a low viscosity liquid in the accessible temperature window.

2. The arrest of pattern formation during a narrow, well defined time-window is essential. While too short exposure to the electric field results in incomplete pattern replication, too long annealing times give rise to coalescence of smallscale structures, degrading pattern fidelity. This is exacerbated by the fact that the direct observation of sub-micrometer patterns in a capacitor slit is nearly impossible and that optical quality control is not scalable.

The purpose of this study is the introduction of an EHL system that combines these two 
requirements. Herein, several low-viscosity resists are exploited for the EHD patterning, including: ethyl celluose (EC), poly(vinyl acetate) (PVAc), poly(vinyl alcohol) (PVA), poly(vinyl pyrrolidone) (PVP) and polycaprolacone (PCL)-diol; their properties are listed in Table 3.1. EC is a commonly available, water insoluble polymer, used in controlled release drug delivery. In its acrylated form it is utilized as a UV-curable ink binder in conductive ceramic inks to control the printing process. PVAc is an amorphous, thermoplastic polymer with a high dielectric constant making it a good gate insulator for organic thin-film transistors. PVA is a biodegradable, water soluble polymer with excellent film-forming, emulsifying and adhesive properties which exhibits a high Young's modulus and can be utilized for rigid templates. PVP is soluble in water and many organic solvents and binds to polar molecules exceptionally well, owing to its polarity. This has led to its application in coatings for photo-quality ink-jet papers and transparencies, as well as in inks for inkjet printers. Its also used as photo resist in color displays for improving picture contrast and image resolution, and as temporary or permanent coating of printed circuit boards.

The use of low-viscosity materials results in a considerable reduction of the completion of EHL to a few seconds only. Low-viscosity epoxy materials were also studied by Dickey et al., who reported similarly low patterning times. [26] A rapid EHD patterning requires precise termination of the pattern formation process. While Dickey and co-workers achieve this by crosslinking of the sample, we demonstrate a method for the in-situ control of the EHL process during this short time span by monitoring the current across the capacitor device. Since the current is passed through the leaky dielectric once it spans the capacitor gap, the overall capacitor current is a good measure for EHL completion. 


\subsubsection{Stability Analysis}

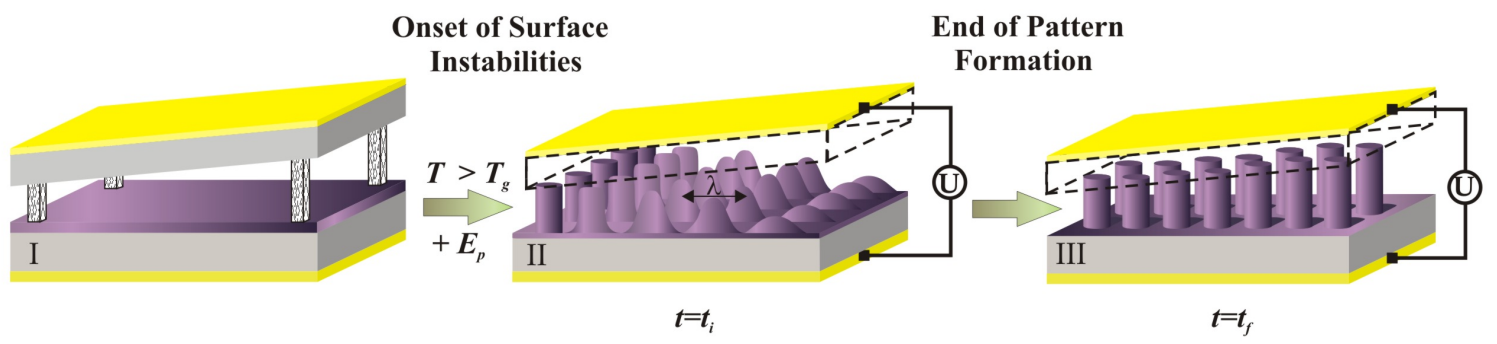

Figure 4.1: Schematic representation of the experimental set-up. The thin film in the capacitor is liquefied by raising the temperature above the glass transition temperature $T_{\mathrm{g}}$ of the resist. The amplification of a capillary surface instability is triggered by applying a voltage $U$. With time the instability causes the formation of liquid bridges between the two plates. The kinetics of pattern formation is observed by either optical microscopy through a transparent electrode or by monitoring the current flowing in the circuit. The time $t_{\mathrm{i}}$ corresponds to the time when the first column span the two plates, while $t_{\mathrm{f}}$ is the time when the column formation process is completed. The wedge geometry of the device is greatly exaggerated in the drawing.

The physical principle underlying the destabilization of thin films by electric fields is well understood. [92, 107, 108, 131, 169, 175, 176] Here, the model describing the earlystage kinetics of pattern formation (linear stability analysis) is briefly summarized. The experiential configuration is schematically shown in Fig. 4.1. A thin resist layer on one electrode is opposed by the second, leaving an air-gap. An applied potential difference $U$ across the two electrodes gives rise to an electric field across the dielectric material and air gap. The dielectric discontinuity at the film-air interface in the capacitor gives rise to the formation of displacement charges which couple to the electric field, causing a destabilizing electrostatic pressure. Following the theoretical analysis of EHD instabilities described in Chapter 2:

$$
p_{\mathrm{el}}=-\varepsilon_{0} \varepsilon_{\mathrm{p}}\left(\varepsilon_{\mathrm{p}}-1\right) E_{\mathrm{p}}^{2}=-\varepsilon_{0} \varepsilon_{\mathrm{p}}\left(\varepsilon_{\mathrm{p}}-1\right) \frac{U^{2}}{\left[\varepsilon_{\mathrm{p}} d-\left(\varepsilon_{\mathrm{p}}-1\right) h\right]^{2}}
$$

where $E_{\mathrm{p}}$ is the electric field in the polymer film, $\varepsilon_{0}$ is the dielectric permittivity of the vacuum, $\varepsilon_{\mathrm{p}}$ is the dielectric constant of the polymer, $h$ is local film thickness and $d$ is the capacitor electrode spacing. While a perfectly homogeneous films is stable, a lateral pressure variation induces in-plane flow. In the lubrication approximation, [101] assuming zero-slip at the substrate and no lateral stresses at film surface

$$
\frac{\partial h}{\partial t}=\frac{\partial}{\partial x}\left[\frac{h}{3 \eta} \frac{\partial p}{\partial x}\right]
$$

where $t$ is the time, $x$ a lateral coordinate, and $\eta$ the viscosity. $p=p_{\mathrm{el}}+p_{\mathrm{L}}$ is the total interfacial pressure consisting of the destabilising electrostatic pressure and the 
stabilising Laplace pressure $p_{\mathrm{L}}=-\gamma\left(\partial^{2} h / \partial x^{2}\right)$ with the surface tension $\gamma \cdot p$ couples to the omnipresent capillary waves of liquid surface, destabilising part of the mode spectrum. This is described by the well-established linear stability analysis, where

$$
h(q, t)-h_{0}=R e \xi \exp \left(i q x+\frac{t}{\tau}\right)
$$

is used with Eq. (4.2). $\tau$ is a characteristic time constant for the temporal evolution of mode $q, \xi$ is the wave amplitude, and $h_{0}$ is the initial film thickness. In the longwavelength limit all non-linear terms are discarded, yielding a dispersion relation (see section 2.2.3) for the wave spectrum

$$
\frac{1}{\tau}=-\frac{h^{3}}{3 \eta}\left[\gamma q^{4}+\frac{\partial p_{\mathrm{el}}}{\partial h} q^{2}\right] .
$$

The exponentially fastest growing mode given by the maximum of Eq. (4.4) eventually dominates the mode spectrum. It has the wavelength $\lambda=2 \pi / q_{\max }$

$$
\frac{\lambda}{\lambda_{0}}=\left(\frac{E_{\mathrm{p}}}{E_{0}}\right)^{-\frac{3}{2}}
$$

with

$$
\frac{\tau}{\tau_{0}}=\left(\frac{E_{\mathrm{p}}}{E_{0}}\right)^{6}=\left(\frac{\lambda}{\lambda_{0}}\right)^{4},
$$

where the following reduced quantities were introduced

$$
\begin{aligned}
q_{0} & =\frac{\gamma}{\varepsilon_{0} \varepsilon_{\mathrm{p}}\left(\varepsilon_{\mathrm{p}}-1\right)^{2} U^{2}} \\
\lambda_{0} & =\frac{2 \pi}{q_{0}} \\
E_{0} & =q_{0} U \\
\tau_{0} & =\frac{3 \eta}{\gamma h_{0}^{3} q_{0}^{4}} .
\end{aligned}
$$

These equations reveal an interesting property of the electrohydrodynamic instability: The variation of the characteristic time constant with electric field is considerably higher compared to the variation of $\lambda$ with electric field. This has the important consequence that very small lateral variations of the electric field (e.g. introduced by a slight variation in the plate spacing $d$ ) have a significant effect on the onset of the instability, while their effect on $\lambda$ may be negligible. Even for a carefully designed experimental configuration the instability does not typically develop everywhere in the capacitor at the same time. Instead an instability front sweeps laterally across 
the sample. This property is exploited in our study to monitor the development and completion of the instability in low-viscosity materials.

To compare the destabilization of materials with differing viscosities, it is useful to introduce an additional set of scaling relations. The inverse of time constant defines a rate for the lateral displacement of material over a distance $\lambda$. Multiplied by $\eta$ this defines a characteristic shear stress

$$
\sigma=\frac{\eta}{\tau}
$$

Defining

$$
\sigma_{0}=\frac{\eta}{\tau_{0}}
$$

introduces the further scaling equation

$$
\frac{\sigma}{\sigma_{0}}=\left(\frac{E_{\mathrm{p}}}{E_{0}}\right)^{-6} .
$$

The data sets in Fig. 4.2A demonstrate the variation of the characteristic wavelength with the electrode spacing for three different polymers: PS, PMMA and bromiated mpolystyrene (PBrS). Fig. 4.2A (top) shows the effect of an increased applied voltage using the same polymer (PS). Due to a higher electric field the electrostatic pressure is augmented and increasingly dominates over the Laplace pressure resulting in a smaller wavelength (squares: $30 \mathrm{~V}$, triangles: $50 \mathrm{~V}$ ).

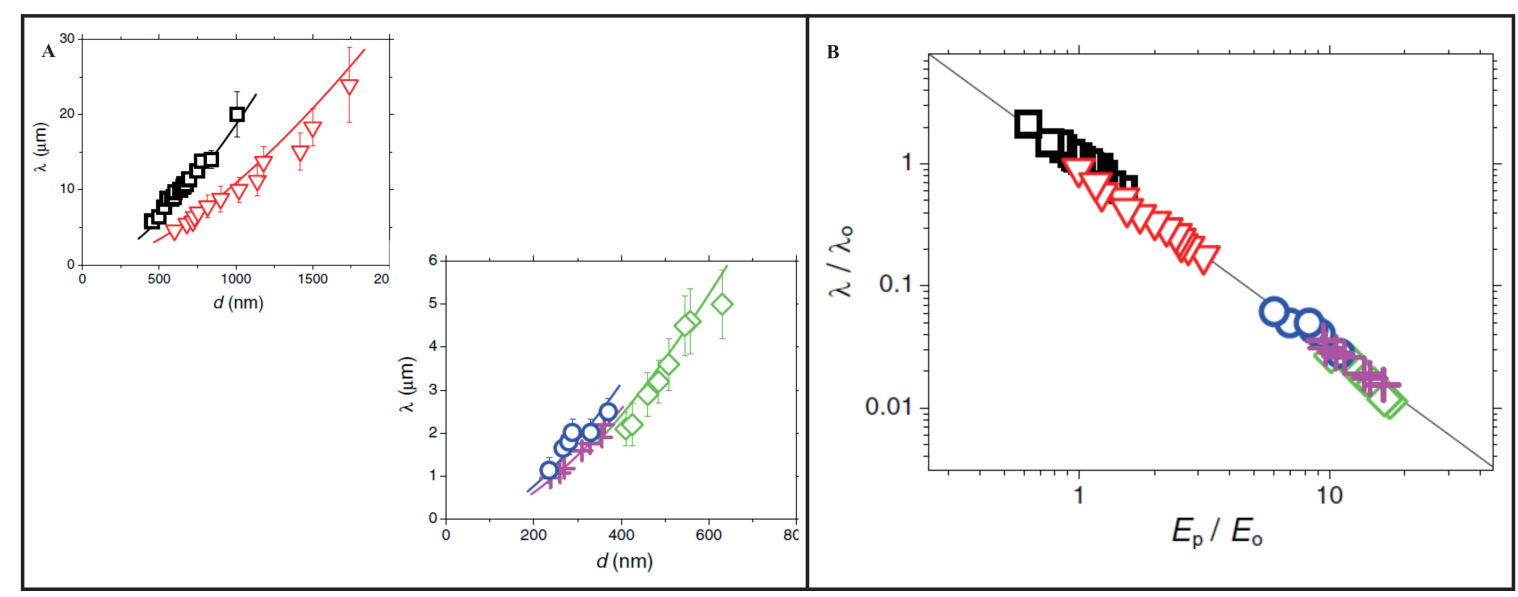

Figure 4.2: (A). Characteristic wavelength vs. electrode spacing Adopted from [127]. The different data sets are referred to by the following symbols: Top: squares: PS with $h_{0}=93 \mathrm{~nm}, U=30 \mathrm{~V}$; triangles: $\mathrm{PS}$ with $h_{0}=120 \mathrm{~nm}, U=50 \mathrm{~V}$. Bottom: circles: PMMA with $h_{0}=100 \mathrm{~nm}, U=$ $30 \mathrm{~V}$; diamonds: $\mathrm{PBrS}$ with $h_{0}=125 \mathrm{~nm}, U=30 \mathrm{~V}$. The crosses correspond to an AC experiment (rectangular wave with a frequency of $1 \mathrm{kHz}$ and an amplitude $U=37 \mathrm{~V}$ ) using a PMMA film with $h_{0}=100 \mathrm{~nm}$. The lines correspond to Eq. (2.37). (B). Variation of the characteristic wavelength vs. the electric field in the polymer in reduced coordinates. All data collapse onto a single curve (Eq. (4.5) without any fit parameters. 
The characteristic wavelength decreases with increasing dielectric constant, for otherwise similar experimental parameters, as can be seen from Fig. 4.2A (bottom) for PMMA and PBrS polymers. Compared to Fig. 4.2A (top) the wavelength for an applied voltage of $30 \mathrm{~V}$ for $\mathrm{PBrS}$ (diamonds) is comparable to $50 \mathrm{~V}$ for PS (triangles). Eq. (4.5) shows that the data can be rescaled in reduced coordinates. Data sets which correspond to a range of experimental parameters (Fig. 4.2A) superpose to a single master curve shown in Fig. 4.2B. Within the experimental scatter, the data is quantitatively described by Eq. (4.5) in the absence of any adjustable parameters. About two orders of magnitude are covered in both dimensionless quantities demonstrating the scaling behaviour in addition to the quantitative agreement.

\section{$4.2 \quad$ Experimental}

\subsubsection{Materials}

All materials were used without further purification. Toluene, chloroform, acetone and isopropanol were purchased from Fisher Scientific. Highly polished $p$-doped silicon (Si) wafers, with $<100>$ crystal orientation (Wafernet GmbH, Eching, Germany) were used as substrates. Electron-beam patterned silicon structures were purchased from X-lith eXtreme Lithography, Ulm, Germany. ITO coated glass slides with a resistance of $112 \Omega / \mathrm{cm}^{2}$ were used as transparent substrates for in-situ optical observations.

\subsubsection{Experimental Procedure}

Thin films with initial thickness of $h=100 \pm 20 \mathrm{~nm}$ were spin-cast onto $1 \mathrm{~cm}^{2}$ silicon substrates from toluene or chloroform solutions with typical concentrations of 2-3\% polymer by weight. Prior to spin-coating, the substrates were cleaned in a 'Piranha' solution consisting of $3: 1 \mathrm{H}_{2} \mathrm{SO}_{4}(98 \%): \mathrm{H}_{2} \mathrm{O}_{2}(30 \%)$, followed by thorough rinsing with deionised water and drying under nitrogen. The ITO-coated glass slides were cleaned by scrubbing in soap water at $75^{\circ} \mathrm{C}$, washing in an ultrasonic bath with acetone and isopropanol, followed by irradiation for 20-30 minutes in an UV-ozone cleaner. Immediately before device assembly, all substrates and electrodes were subjected to snow-jet cleaning. Capacitor devices were assembled using a film-covered substrate as lower electrode. Facing it, planar or topographically structured silicon wafers were mounted leaving a thin air gap. The overall electrode spacing $d$ was adjusted using silicon oxide colloids as spacers. A slight misalignment of the two electrodes resulted in a slight wedge geometry leading to a variation of $d$ from $\sim 100 \mathrm{~nm}$ to $\sim 1 \mu \mathrm{m}$ across the entire sample width of $1 \mathrm{~cm}$. Both electrodes were electrically contacted using silver paint (Electrodag 1415M). 
The films were liquefied by thermal annealing: the temperature was raised above the $T_{\mathrm{g}}$ of the resist, and a voltage of $40 \mathrm{~V}$ was applied to the capacitor device. The experiment was terminating by quenching the assembly to room temperature before removing the electric field. Current curves were recorded during each EHD patterning process. A sparse distribution of sample imperfections and the fact that the silica colloids are wetted by the polymer gave rise to a small current prior to film patterning. $t=0$ marks the time the voltage is applied to the capacitor. The slight offset in current recording arose from a delay in current recording. Representative current curves exhibit typical sigmoidal shape as function of time while defining the onset and termination times of the EHD patterning process are shown in Fig. 4.5.

After each experiment, the top electrode was removed and the formed pattern was analysed using optical microscopy, atomic force microscopy and scanning electron microscopy.

\subsubsection{Characterization}

The evolving patterns were observed in-situ by an inverted optical microscope. The smallest visually detectable amplitude fluctuation in the film $(\sim 5 \mathrm{~nm})$ determined the onset of pattern formation. A NanoScope IV Multimode and a Dimension 3100 AFM was employed to quantitatively determine the sample topography. All the measurements were performed in air at ambient conditions using the tapping mode. NSG 20 cantilevers with a resonance frequency of $260 \mathrm{kHz}$ and a stiffness of $28 \mathrm{~N} \mathrm{~m}^{-1}$ were used. Height and phase images were analyzed with the Nanoscope software (Digital Instruments). The AFM measurements yielded the film thickness $h$, the plate spacing $d$ (by measuring the height of the generated structures relative to the initial film thickness), the characteristic wavelength $\lambda$, and width and height of the replicated patterns. Scanning electron microscopy (SEM) was used to support the AFM measurements. A LEO ULTRA 55 SEM with a Schottky-emitter ( $\mathrm{ZrO} / \mathrm{W}$ cathode) with an acceleration voltage of $10.0 \mathrm{kV}$ was used. The resist viscosities were measured using a $1^{\circ}$ cone and plate rheometer (Physica MCR 501, Anton Pear) at temperatures above the $T_{\mathrm{g}}$ of each polymer. A constant-voltage source was used to apply a voltage of $40 \pm 1 \mathrm{~V}$ across the capacitor devices and the resulting current was measured using a Keithley Model 2000 multimeter. 


\subsection{Results and Discussion}

\subsubsection{Homogeneous Electric Field Induced EHD Pattern For- mation in Low-Viscosity Materials}

To demonstrate the suitability of low-viscosity polymers, pattern formation and replication was studied first. Approximately $100 \mathrm{~nm}$ thick resist layers were spin-cast onto silicon wafers, which were opposed by a second silicon wafer, leaving an air-gap. The details of device assembly and characterization are given in the Experimental Section and in Chapter 3. The employed materials are summarized in Table 3.1. Two device geometries were employed as previously described: capacitors with planar and structured top electrodes. The use of an unstructured top electrode resulted in the formation of a locally hexagonal array of columns shown in Fig. 4.3.
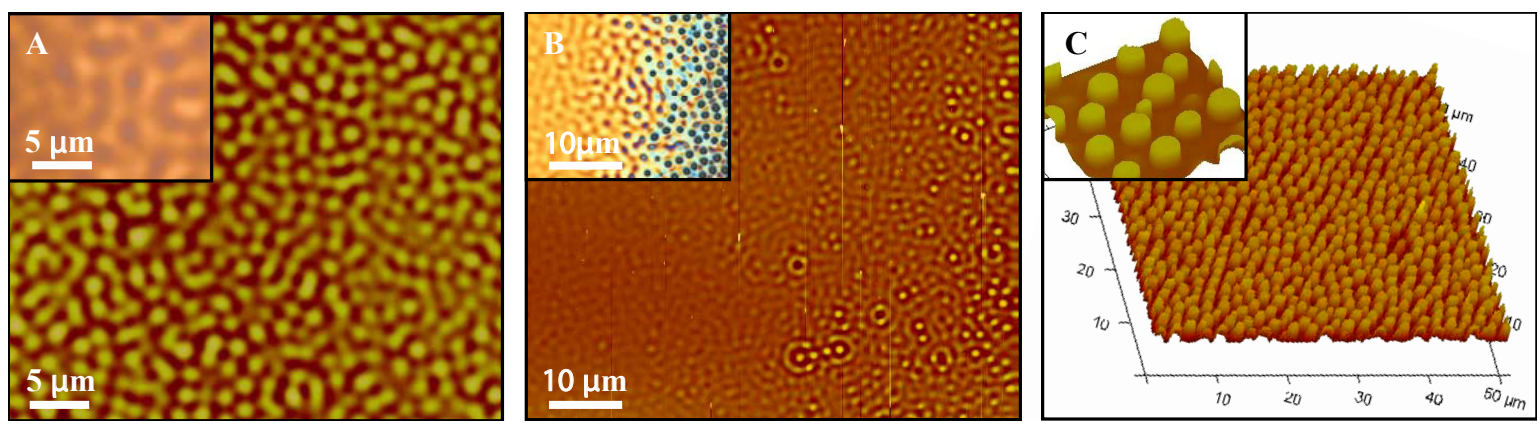

Figure 4.3: AFM micrographs of the instability evolution of a $100 \mathrm{~nm}$ thick PVAc film in a homogeneous electric field. (A) Initial surface undulation after $7.0 \pm 4 \mathrm{~s}$, evolving into $250 \pm 17 \mathrm{~nm}$ high columns with a diameter of $\sim 2 \mu \mathrm{m}$ and an average inter-column spacing of $2.5 \pm 0.5 \mu \mathrm{m}$ in $(\mathrm{C})$. The intermediate stage in (B) (inset: optical micrograph) shows the consequence of a slight variation in the inter-plate spacing, showing a range of stages of the instability (left: undulations, right columns). While the lateral variation of the electric field has a strong effect on the destabilization kinetics, its effect on the pattern that evolves is negligible (see text).

For all studied materials, the instability proceeded in the same fashion: the initial amplification of a low-amplitude capillary wave in Fig. 4.3A resulted in an array of columns spanning the two electrodes in Fig. 4.3C. The initially observed wavelength in Fig. $4.3 \mathrm{~A}$ of $2.5 \mu \mathrm{m}$ as well as the final inter-column spacing are in good agreement with the stability analysis outlined in the previous section. Despite the much shorter destabilization time of $\sim 7 \mathrm{~s}$, the pattern formation process of Fig. 4.3 is similar the well-studied case of generic polymers, [49, 50, 96] confirming the same underlying physical mechanism. Figure $4.3 \mathrm{~B}$ is an intermediate snapshot revealing the pattern formation kinetics. While the pattern on the left hand side of the image is reminiscent of the early stage instability of Fig. 4.3A, full column formation is seen on the righthand side of the image. The origin of this lies in the small lateral variation of the 
capacitor plate spacing $d$ (by a few $\mu \mathrm{m}$ per $\mathrm{cm}$ ). Because of the scaling of $\tau$ with the sixth power of the electric field (Eq. (4.5)), this results in an instability wave propagating form the right part of the sample (small $d$ ) to the left. The much lower dependence of the wavelength on the electric field of Eq. (4.4), on the other hand, explains that the lateral variation of the final pattern (Fig. $4.3 \mathrm{C}$ ) is only very small.

\subsubsection{EHL Pattern Replication of Low-Viscosity Resists in a Heterogeneous Electric Field}
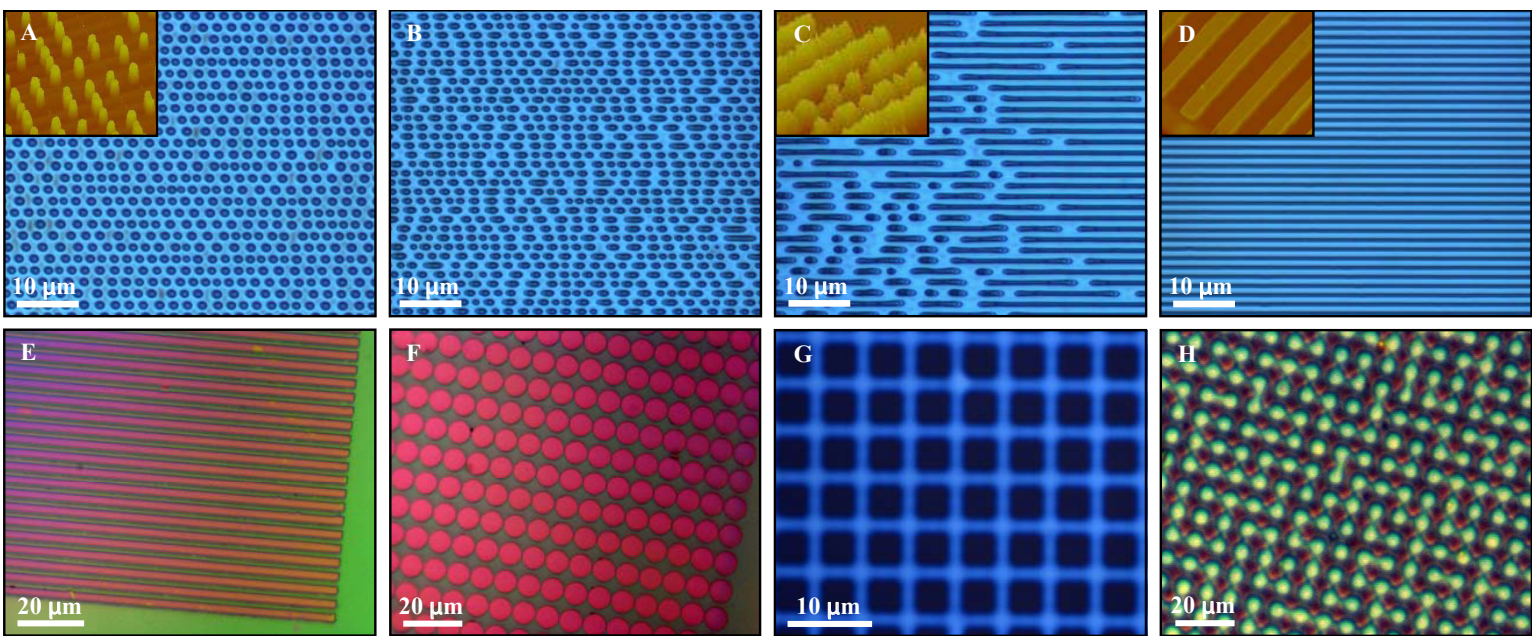

Figure 4.4: Optical micrographs of pattern formation in EC films in laterally heterogeneous electric fields. (A)-(D) Pattern replication of a line grating, imaged after immobilisation of the thin film and removal of the master electrode. An overall wedge geometry allowed to capture different stages of the pattern replication process $(h=100 \mathrm{~nm}, U=40 \mathrm{~V}, d$ decreasing from left to right). (A) Initial capillary plugs spanning the substrate and protruding lines, (B)-(C) increasing coalescence of the capillary plugs, (D) final replicated grating. Corresponding AFM images are shown in insets. (E)-(H) High fidelity EHD replication in an EC films showing the robustness of pattern replication with respect to the parameters of the electrode.

The use of a patterned top electrode gives rise to a laterally varying electric field. For air-gap widths larger than the film thickness and for high pattern densities on the opposing electrode, the initial phase of the instability is similar to the case of Fig. 4.3A. Fig. 4.4 A-D shows the evolution of pattern formation following this initial stage. Starting from an initial homogeneous undulation, the wave pattern couples to the lateral field variation and the further growth of the instability is focused in the direction of the highest electric field and is thereby guided towards the protruding patterns of the top mask. This gives rise to a pillar pattern spanning the substrate and the top electrode at locations of smallest inter-electrode spacing (Fig. 4.4A), which by coalescence (Fig. 4.4B,C) eventually forms positive replica of the master electrode (Fig. 4.4D). In difference to the case shown in Fig. 4.3, the coalescence of the capillary 
plugs bridging the substrates and the protruding parts of the top electrode proceeds rapidly, forming an integral part of the pattern replication kinetics. As shown before, [166] the seamless sequence of capillary instability followed by the coalescence of the initial capillary bridges gives rise to the near-perfect replication of a master pattern, which is quite robust with respect to the size and periodicity of the paster pattern and is not visibly influenced by the small overall wedge geometry of the capacitor, as shown in Fig. 4.4D-H. Once the correct sample parameters are identified, high fidelity pattern replication over the entire electrode area $\left(100 \times 100 \mu \mathrm{m}^{2}\right.$ in our case) can be obtained. In contrast to the previously EHD patterned high viscosity polymers by N. Voicu, [166] the advantage of using a low viscosity polymer is a completion of the entire process in less than $10 \mathrm{~s}$. The patterning time does not depend on sample size and is limited only by the ability of creating a capacitor geometry with sufficient precision in inter-plate spacing.

\subsubsection{Current Monitoring of the Rapid EHL Process}

The relatively high speed of the EHD process sets a strict time limit for the termination of the pattern formation process, since too long exposure of the liquefied film to the electric field may lead to undesired coarsening of the pattern by coalescence. A convenient way to follow the progress of pattern formation and replication involves monitoring the current across the sample. Most dielectric organic resists conduct current in their liquid state, mostly mediated by ionic impurities in the materials. [131] For $100 \mathrm{~nm}$ thick films (see Experimental Section), this current is on the order of $10 \mathrm{~mA} / \mathrm{cm}^{2}$. For the patterned materials of this study the time-evolution of the current can be sub-divided into three regimes: an initial current of several $\mathrm{mA} / \mathrm{cm}^{2}$, an intermediate regime during which the current increases and a final plateau of $\sim 10 \mathrm{~mA} / \mathrm{cm}^{2}$.

In-situ optical investigation using a transparent electrode was used to correlate the structure formation process with the measured current. Current traces correlated with the evolving EHD topography are shown in Fig. 4.5. To note, that the calculation of the current is non-trivial, since it's requires a detailed knowledge of stochastic charge transport mechanisms in thin films. The initial current corresponds to the sample before pattern formation sets in, mediated by the spacer particles. The onset time of the current rise $t_{\mathrm{i}}$ coincides with the time when the the first columns bridge the two plates at the location of smallest plate spacing (right-hand side of Fig. 4.3B). 


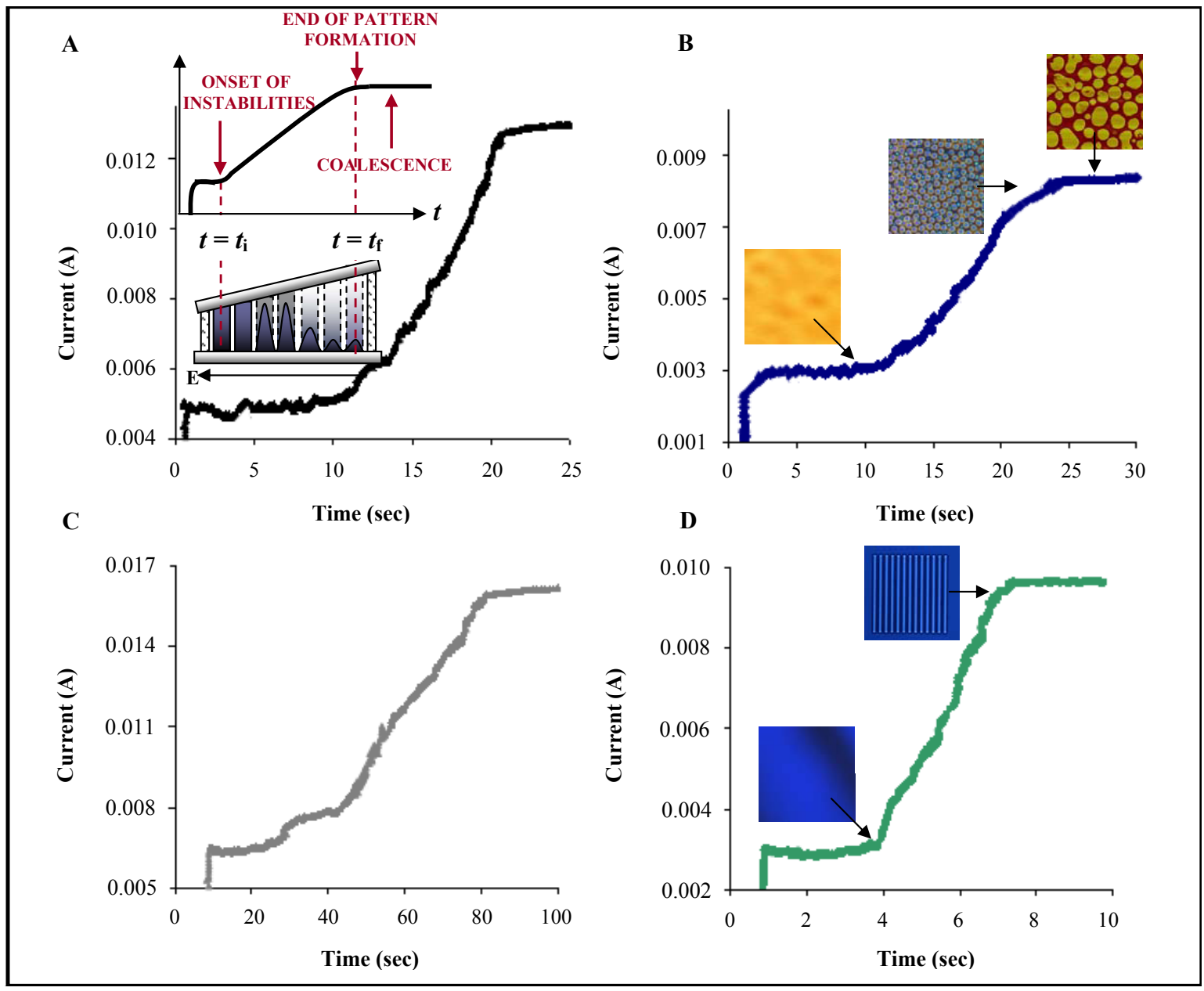

Figure 4.5: Short-circuit current across capacitor devices as a function of time during the EHD patterning process. (A)-(C) PVA, PVAc and PVP films, accordingly, in a homogeneous electric fields with viscosities of $0.073 \mathrm{Pas}, 0.225 \mathrm{Pas}$, and $1.032 \mathrm{Pas}$, respectively. Insets: (A) schematic representation of a typical S-curve for current flow through the capacitor device during patterning, indicating the onset and the termination of the experiment; schematic representation of an exaggerated wedge geometry, demonstrating the correspondence between pattern evolution and current curves as a function of the plate spacing.(B) optically observed patterns during the initial and intermediate stages of pattern formation and and AFM image of the late stage of the process, where coalescence has coarsened the initial pattern (indicated by arrows). (D) EC with $\eta=0.084 \mathrm{~Pa}$ in a laterally varying electric field caused by a topographic grating. The insets show optical images of the sample a at the various stages. The parameters of all measurements were $h \approx 100 \mathrm{~nm}, d \approx 250 \mathrm{~nm}$ (average), $U=40 \mathrm{~V}$, and $T=135^{\circ} \mathrm{C}$.

Because of the exponential variation of the local film thickness with time (Eq. (4.3)), $t_{\mathrm{i}}$ can be approximated by the characteristic time $\tau$ of Eq. (4.6) for this plate spacing. As the instability front sweeps across the sample, the number of capillary plugs increases, thereby increasing the short-circuit current. The current saturates $\left(t=t_{\mathrm{f}}\right)$ once the entire sample is covered by capillary bridges, which is comparable to the value of $\tau$ for the largest value of $d$ of the sample. For the sample consisting of two planar electrodes, pattern coarsening does not lead to a further current increase, because the 
integral cross-sectional area of the capillary plugs does not increase. The interpretation of the current increase in the case of pattern replication is slightly more complex. While the same basic principle of the initial pattern forming process of the previous paragraph also applies here, the initial columns are localized at lateral positions where the top-electrode protrudes downwards to the film (Fig. 4.4A). These columns are laterally connected to unpatterned parts of the resist at locations of lower electric fields. [166] With time more material is drawn into the columns at high electric field locations, leading to an increase in their diameter and eventually to their coalescence (Fig. 4.4B,C), and eventually to the replication of the electrode structure (Fig. 4.4D). The current increase is therefore a convolution of the instability front that laterally sweeps across the sample with this coarsening process. While $t_{\mathrm{i}}$ is comparable with $\tau$ for the location of smallest $d$ of the sample, $t_{\mathrm{f}}$ signifies the time when the entire pattern replication process is terminated. Characteristic current traces of a sample with a patterned top-electrode is shown in Fig. 4.5D.

\subsubsection{Variation of the Instability Time Constant with the Vis- cosity}

The onset and the pattern completion characteristic time constants were experimentally measured by both monitoring the current trace across the sample and an in-situ optical examination via a transparent electrode during the EHD patterning process. Figure $4.5 \mathrm{~A}$ shows the variation of the film destabilization time with the viscosity of the polymer. In order to compare sample with different experimental parameters, the data is plotted as a function of $\eta / \sigma$, where all other experimental parameters are absorbed in the shear stress $\sigma$. While the onset of the current increase $t_{\mathrm{i}}$ is relatively well described by the prediction of Eqs. (4.6)and (4.10), the optically detected onset of the instability and the completion of patterning $t_{\mathrm{f}}$ is offset towards higher values compared to the prediction of $\tau$. While the first observation, can be possibly explained by the expected higher (human) error involved in the optical detection in comparison to the current measurements, indication that the completion of patterning systematically exceeds that the longest time constant of the system requires further investigation. 

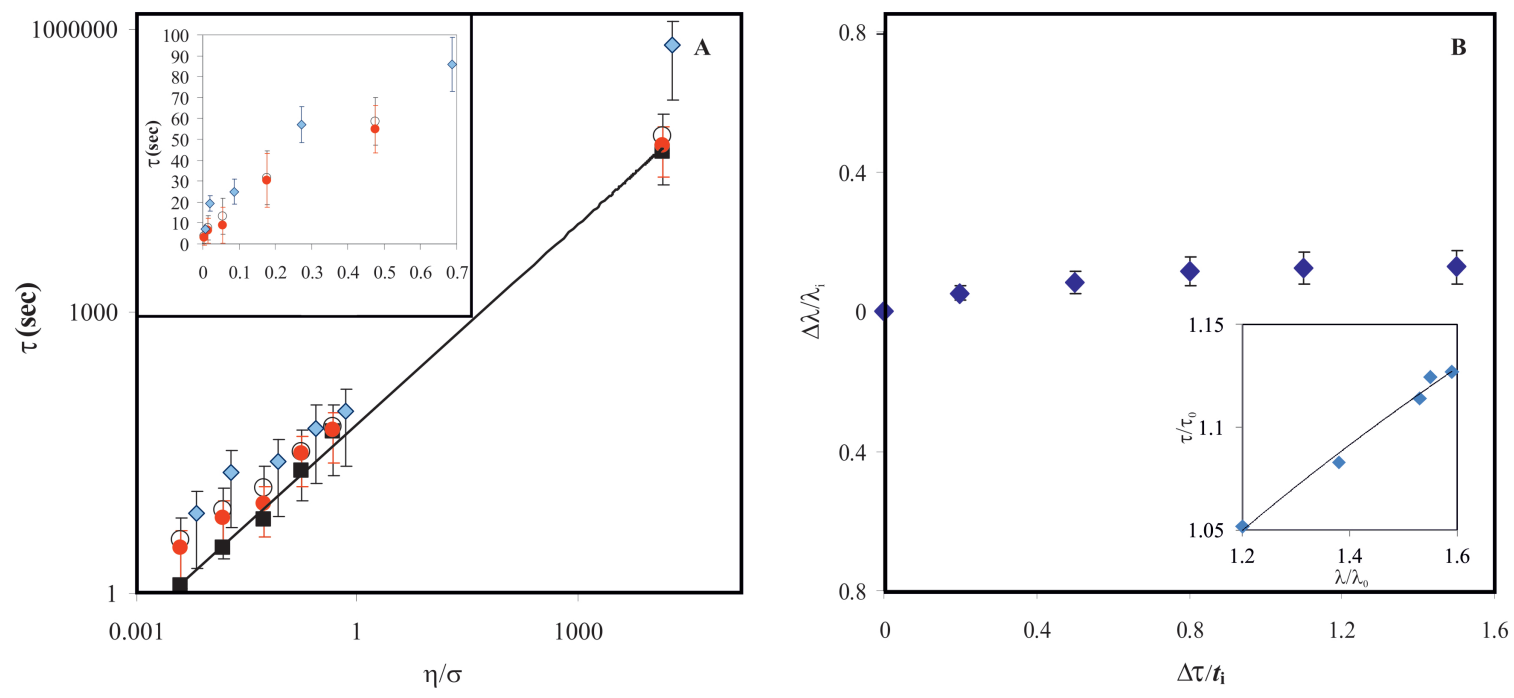

Figure 4.6: (A) Variation of the the instability time constant $\tau$ with viscosity in terms of Eq. (4.8). The data obtained by current monitoring (full red circles: $\tau=t_{\mathrm{i}}$ ) agree within their error margins and are well described by the prediction of Eq. (4.8) (line). Optically determined values of $\tau$ (open circles) systematically (slightly) exceed the predicted values. The data obtained from the termination times (diamonds, $\tau=t_{\mathrm{f}}$ ) follow the same trend, but are offset towards longer times. (B) Variation of pattern periodicity $\Delta \lambda / \lambda_{\mathrm{i}}=\left(\lambda-\lambda_{\mathrm{i}}\right) / \lambda_{\mathrm{i}}$ as a function of the variation of the patterning time $\Delta \tau / t_{\mathrm{i}}=\left(\tau-t_{\mathrm{i}}\right) / t_{\mathrm{i}}$ for a PVAc film $\left(\lambda_{\mathrm{i}}=\lambda\left(\tau_{\mathrm{i}}\right)\right)$. Over a lateral distance of $45 \mu \mathrm{m}$, the wavelength changed only by $12 \%$ compared to a variation of $\Delta \tau / t_{\mathrm{i}}$ by a factor of 2.5. Inset: The power law relation of $\left(\tau / \tau_{0}\right)=\left(\lambda / \lambda_{0}\right)^{4}$.

As qualitatively extracted from Fig. 4.3, the marked temporal evolution of the instability caused by small inhomogeneities in the electric field has only a minor influence on the structural parameters of the instability (i.e. $\lambda$ ). This is quantitatively shown in Fig. 4.6B. For a PVAc film, $\lambda$ changed by only $12 \%$ over a lateral distance of $45 \mu \mathrm{m}$, despite a relative variation of the time constant by a factor of 2.5 . This is a consequence of the power-law relations of Eqs. (4.4)-(4.5), with $\tau$ and $\lambda$ differing in their response to variation in the electric field by 4 orders of magnitude.

Finally, we address the question in how far the small but finite current across the device violates the assumption of the linear stability analysis, modeling the polymer layer as a perfect dielectric. A finite polymer conductivity lowers the electric field in the layer and increases the electrostatic stress at the surface, as predicted in the leaky dielectric model by Pease and Russell. [107, 108, 175] While this marginally alters $\lambda$, its effect on the $\tau$ may be significant (again, because of the higher order scaling of $\tau$ with the electrostatic surface pressure). The fact that the time constants in Fig. 4.6 are well described by a model neglecting the finite conductivity suggests the suitability of the more simple model used in our data analysis. 


\subsection{Conclusions}

In summary, we have explored the use of low viscosity materials for fast EHD patterning. In difference to the typically published polymers, the studied materials not only have a thermally accessible glass transition temperature but also exhibit very low viscosities upon softening. This enables the completion of the EHD process within seconds rather than hours, increasing the technological appeal of this technique. The rapid rate of the EHD instability demonstrated in these experiments, requires however, an improved monitoring method of the patterning process. We have therefore, exploited the small but finite current that flows through the device during pattern formation. The current trace has a sigmoidal shape (as function of time) defining the characteristic onset and completion time of the EHD process. While $t_{\mathrm{i}}$ corresponds closely to the characteristic time of the instability $\tau, t_{\mathrm{f}}$ is primarily of practical interest, indicating the completion of pattern formation and replication. From a practical point of view, it is essential that the sample is solidified at $t \approx t_{\mathrm{f}}$ to prevent a deterioration of the pattern by coarsening. Because of the quick pattering time, rapid sample quenching is important, possibly requiring a sample support that allows in-situ cooling. Importantly, our study shows that, despite the much shorter patterning times, the EHD process is similar to that of high viscosity polymers and is therefore well described by a linear stability analysis.

This technique should be easily extendable to feature sizes down to 10 nanometres. Reducing for example the interfacial surface tension further accelerates pattern replication and reduces the characteristic pattern size. In the lithographic mode, electrostatically induced structure formation is not limited to the intrinsic wavelength, allowing the replication of a large range in feature sizes for otherwise identical experimental parameters. Reducing the replicated feature size, further decreases the time constant to a millisecond and below. In this accelerated mode, EHD lithography is a promising prospect for a rapid, straightforward, low-cost replication technique of sub-micron patterns. 


\section{Alignment of Carbon Nanotubes via}

\section{EHD-Driven Patterning of Nanocomposites}

The ability to design, manufacture and control highly reliable and robust submicron patterns is of profound interest for high-performance integrated nano devices. Owing to their exceptional electric, thermal and mechanical properties, carbon nanotubes (CNTs) have been a topic of extensive research, in particular because of their potential incorporation into easily processable and flexible polymer matrices. [12, 45, $73,104,125,137,154$ ] In order to take full advantage of the synergistic functions of CNT nanocomposites, the control of dispersion, orientation and interfacial chemistry of CNTs in the organic or inorganic matrix is required. Moreover, anisotropic composite structures with vertically aligned CNTs are essential to realize the full potential of nanotube-based composites both for optimization of their mechanical properties and integration into devices.

This study presents a route towards controlled reproducible alignment of CNTs during an EHD patterning process. EHD patterning of carbon nanotube-polymer composite films yields well-defined patterns on the micrometer scale along with the alignment of CNTs within these patterns. Conductive pathways in nanotube networks formed during EHD patterning of nanocomposite films results in a substantial increase in the composites' conductivity at loadings (i.e., weight fractions) exceeding the percolation threshold. Furthermore, the degree of nanotube alignment can be tuned by adjusting the EHD parameters and the degree of alignment is mirrored by the conductivity across the film. Using an etching techniques or by embedding relatively long nanotubes, patterned surfaces decorated by CNT brushes are generated in this study.

\subsection{Introduction}

\subsubsection{Carbon Nanotubes: Structure and Properties}

The discovery of CNTs by Iijima more than two decades ago, not only revealed a novel and unique structure of carbon, but also attracted a considerable scientific and 
technological interest. CNTs are allotropes of carbon having $\mathrm{sp}^{2}$ hybridization. They are hydrophobic and do not dissolve in water or any other polar solvents. Additionally, due to their high molecular weight and strong inter-tube interactions, they are insoluble in most organic solvents.

There are two major types of CNTs: single-walled nanotubes (SWNTs) consist of a single graphite sheet seamlessly rolled up into a cylindrical tube and multi-walled nanotubes (MWNTs), comprises of several such nanotubes in a concentric assembly (Fig. 5.1B). The diameters range of SWNTs is $1.0-1.4 \mathrm{~nm}$ and $2.5-70 \mathrm{~nm}$ for MWNTs. The nanotube length varies from a few tens of nanometers to several micrometers, corresponding to extremely high aspect ratios. This high aspect ratio is responsible for many of their exceptional properties. The combination of the high aspect ratio of the nanotube on one hand and the high rigidity (large persistence length) on the other provides an extraordinary balance between strength, stiffness, flexibility and low weight.

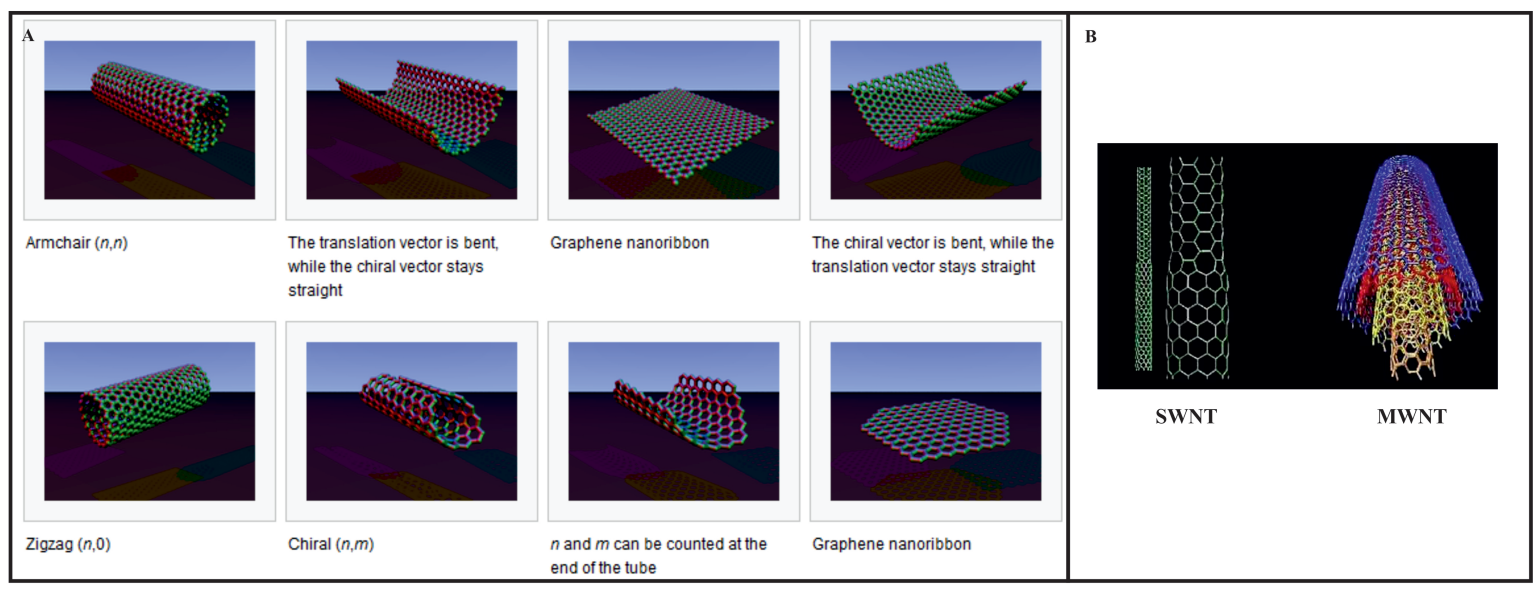

Figure 5.1: The structure of a SWNT can be conceptualized by wrapping a one-atom-thick layer of graphite called graphene into a seamless cylinder. Adopted from [2]. The way the graphene sheet is wrapped is represented by a pair of indices $(n, m)$. The integers $\mathrm{n}$ and $\mathrm{m}$ denote the number of unit vectors along two directions in the honeycomb crystal lattice of graphene. If $m=0$, the nanotubes are called zigzag nanotubes, and if $n=m$, the nanotubes are called armchair nanotubes. Otherwise, they are called chiral. (B). SWNT vs. MWNT. Adopted from [4].

Although SWNTs and unfolded sheets of graphite have structural similarities, a sheet of graphite is an electric conductor while, SWNTs can be either metallic or semiconducting. The way in which the graphite sheet is joined to form a nanotube determines the armchair, zigzag or chiral nanotubes forms, which have different electrical properties (Fig. 5.1A). The conductance in nanotubes occurs through discrete electronic states due to the nanometric size of the nanotubes. Also, because of the geometric confinement in the radial direction the electrons are forced to move only along the nanotubes axis. Consequently, the electron transport along the hollow cylindrical 
core is ballistic with low resistivity and very low heat dissipation. The electronic properties of MWNTs are quite similar to those of SWNTs because of the weak coupling between the coaxial cylinders. The tensile strength of carbon nanotubes is above $1 \mathrm{TPa}$ and the elastic modulus is a few tens of GPa, which is one order of magnitude higher than that of carbon fibers.

The extraordinary electro-mechanical properties have inspired a host of potential applications, ranging from large scale structures in automobiles to nanometer-scale electronics.

\subsubsection{EHD Lithography: A Route Towards Well-Defined CNT Structures in a Patterned Nanocomposite}

CNTs have been introduced into several host materials (polymer, ceramics, metal) to enhance the overall properties of the CNT composite system. While the combination of discrete CNTs as fillers in polymers to form nanocomposites [67] results predominantly in the reinforcement of the material, the use of CNTs as scaffold for the synthesis of inorganic replica can be used to manufacture hybrid materials, which have a range of interesting properties. [32] In both systems, bottom up strategies have been exploited to generate new classes of high-performance nanotube-based composite and hybrid materials, whose properties can be specifically tailored for the desired application. Nanotube-based polymer composites have potential for use in a wide range of applications, including electronic devices, solid state sensors, [137, 172] electrical interconnects, high thermal conductivity heat sinks [35] and field-effect transistors. [151] CNT nanocomposites and hybrids also been successfully applied in solar cells, $[74,75,120]$ as photocatalysts for hydrogen production, [25] and as anode materials [98] and additives [33] in lithium-ion batteries and super-capacitors. Moreover, while Li et al. demonstrated that the incorporation of CNTs in a P3HT layer [81] increased the photo-conversion efficiency in a photovoltaic device caused by a faster electron transport via the nanotube network, the performance can probably be further improved by using three-dimensional (3D) architectures with vertically aligned CNTs. In particular, nanopillar array photo-electrodes enhance the optical absorption efficiency $[59,147]$ by enhanced collection of low-energy photons absorbed far below the surface, as compared to planar photoelectrodes.

Exfoliation (i.e., de-bundling and dispersion) routes of CNTs have been studied extensively, showing that a uniform and stable dispersion can be governed by covalent or non-covalent interactions between hydrophobic CNTs and the dispersant molecules. $[30,54,61,90,95,112,124]$ Researchers have also managed to successfully align CNTs in liquid solutions upon applying an external force (e.g., electric or magnetic field, 
mechanical stretching, etc). [7, 57, 70, 155] While some horizontal orientation can be achieved when CNTs are spin-cast into a thin film from a pre-aligned liquid-crystalline solution, a near complete loss of orientation is typically obtained upon their incorporation into a host matrix. [27, 80, 111] Hence, achieving a vertically oriented nanotube network in a fully-processed composite remains an important challenge. There is presently no technique to produce a well-defined CNT structure in a nanocomposite, which provides long-range control of feature positions combined with the control over CNT orientation in the composite.

In the present work we introduce a simple, versatile EHD lithographic technique for patterning CNT-based polymer composites. This method provides a straightforward, single-step approach for generating high-fidelity micron-sized nanocomposite structures within which anisotropic CNTs are vertically aligned. The EHD concept makes use of the destabilization of thin polymer films by means of an electric field. Placing a liquified resist between parallel plates, leaving a small air gap, and applying a potential gives rise to a homogeneous electric field in the polymer layer, $E_{\mathrm{p}}$, which causes the formation of well-defined pillars with local hexagonal symmetry spanning the capacitor gap. In the presence of laterally varying $E_{\mathrm{p}}$, i.e. when a topographically patterned top electrode is used, the EHD patterning process yields a high fidelity replication of the patterns imposed by the master top electrode. EHD lithography has previously been used to fabricate patterns in a variety of polymers using both featureless and topographically structured masks. [42, 130] Furthermore, EHD lithography allows to pattern most of the amorphous and semi-crystalline polymers. [42] Positional control of the generated morphologies can be adjusted by varying a number of experimental parameters, such as the initial film thickness, inter-electrode spacing, $E_{\mathrm{p}}$-strength, surface tension and lateral periodicity of the master electrode. The degree of CNT orientation is governed by key process parameters such as the hydrodynamic flow and the strength of the applied electric field. The possibility to control self-organized patterns on the micrometer scale and the degree of anisotropy of the incorporated CNTs within the patterned nanocomposite renders this technique technologically appealing.

\subsection{Experimental}

\subsubsection{Materials}

CVD grown multi-wall carbon nanotubes (MWCNTs) (outer diameter: 8-15 nm, purity: higher than 95\%) were purchased from Cheap Tubes Inc. Polystyrene, (PS) (molecular weight: 100 and $300 \mathrm{kgmol}^{-1}$, polydispersity $<1.07$ ), was purchased from Polymer Standards Service GmbH, Mainz. Highly polished $p$-doped silicon (Si) wafers, 
with $<100>$ crystal orientation (Wafernet Gmbh, Eching, Germany) were used as substrates.

\subsubsection{Experimental Procedure}

The dispersion of MWCNTs in PS is facilitated by the high wetability of the aromatic PS on the graphitic MWCNT surface [112]. MWCNT-PS composites were prepared using two established procedures: a solution-evaporation method assisted by high-energy sonication, $[112,123]$ or block polymer stabilization of nanotubes with further incorporation into a PS matrix. [142] Both processes yielded stable and uniform dispersions of nanotubes inside a PS matrix. For EHD experiments, a capacitor was assembled using a silicon substrate covered by a homogeneous nanocomposite film as a lower electrode. Facing it, a planar or structured Si-wafer was mounted as second electrode, leaving a thin air gap. Both capacitor electrodes were electrically contacted using silver paint (Electrodag 1415M). In some of the EHD experiments, the upper electrode was substituted by a thick Kapton CR (DuPont) sheet with a thermally evaporated $50 \mathrm{~nm}$ thick $\mathrm{Au}$ layer on the reverse side. In order to avoid the air gap and establish conformal contact with the composite film, a thin layer of poly(dimethylsiloxane) (PDMS, Sylgard 184, Dow Corning) was spin-cast and thermally cross linked on the front side of the Kapton sheet. Since, the presence of a thick Kapton layer and a dielectric, significantly lowers the effective field across the nanocomposite layer, a much higher voltage of $4 \mathrm{KV}$ was applied across such an assembly. Etching in an air plasma was used to degrade PS and to partially expose the embedded MWCNTs.

\subsubsection{Characterization}

A quantitative analysis to determine the average degree of MWCNT alignment inside the generated columns was carried out employing eye correlations Scanning electron micrographs were obtained using a LEO ULTRA 55 SEM with a Schottky-emitter ( $\mathrm{ZrO} / \mathrm{W}$ cathode). The acceleration voltage was $15.0 \mathrm{kV}$ and secondary electron images were recorded at various magnifications. Cross-sectioned surfaces for SEM investigations were prepared by fracturing the pillar array, with the fracture plane parallel to the pillar axis. A Dimension 3100 atomic force microscope (Bruker) was employed for AFM imaging to characterize patterned composite films and their cross-sections. All the measurements were performed under ambient conditions using the Tapping Mode. Golden Silicon Probes cantilevers (NSG 20 with resonance frequency of $260 \mathrm{kHz}$ and a force constant of $28 \mathrm{Nm}^{-2}$ ) were used. For TEM cross sectional imaging, a thin layer of Pt was sputtered onto the composite films, followed by embedding into a Spurr epoxy resin. The substrate was removed and the films were sectioned using diamond knife in 
a Leica Ultracut Microtome, yielding sections with a thickness of $50 \mathrm{~nm}$. The crosssections were analyzed using a FEI Technai 20 TEM at acceleration voltage of $120 \mathrm{kV}$. The resistivity of the epoxy-embedded films was measured by a four-probe method using a Keithley 2000 voltage-current source.

\subsection{Results and Discussion}

\subsubsection{Dispersion of CNTs and Their Incorporation Into the Polymer Matrix}

An essential requirement for EHD patterning of CNT nanocomposites is a uniform and stable dispersion of CNTs in the host polymer matrix. Polystyrene (PS) was used as the matrix polymer as a proof of the concept, but nearly any polymer can be used in a similar manner.

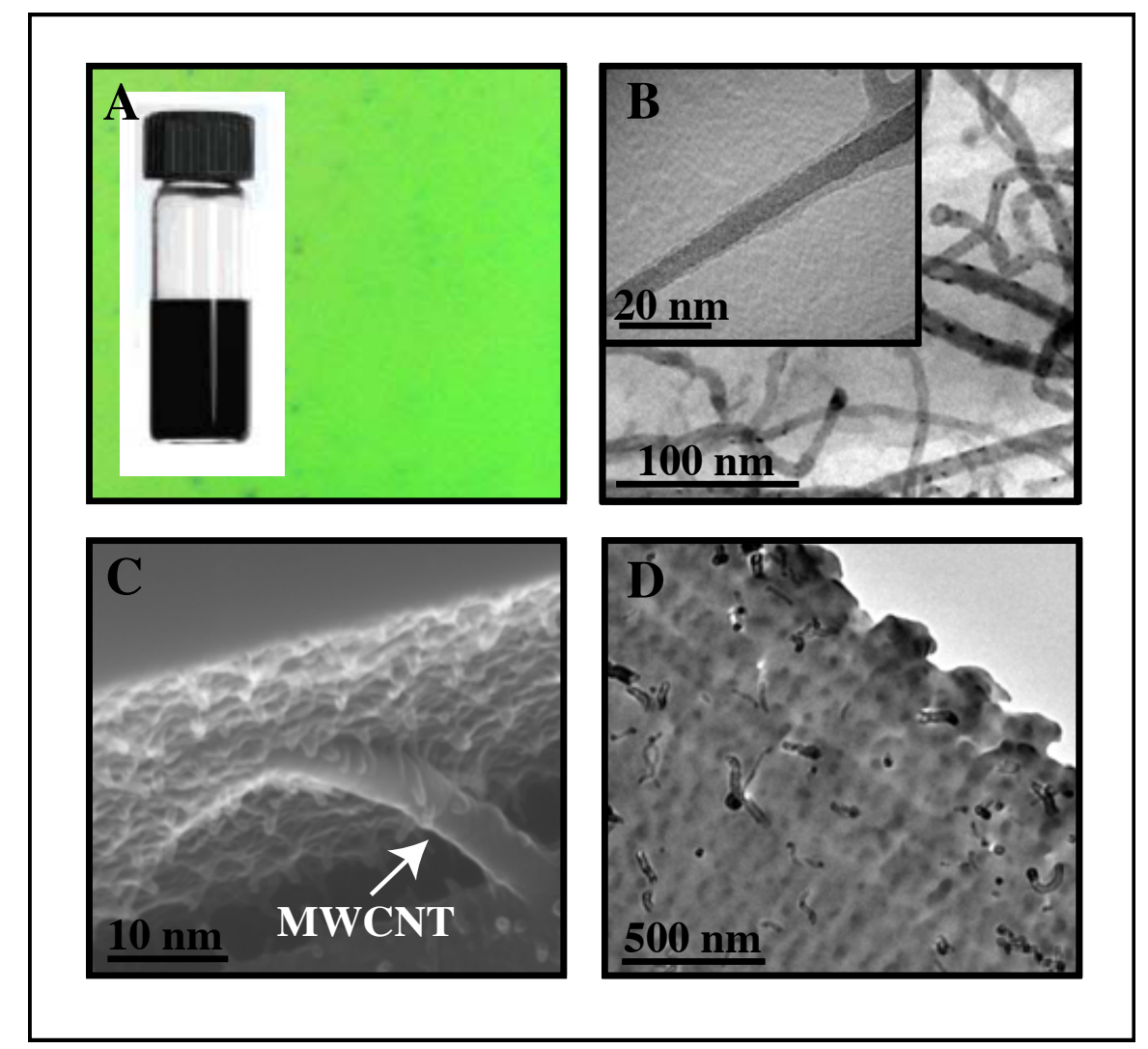

Figure 5.2: Overview of the CNTs-polymer composite prior to the EHD experiment: (A) Optical micrograph of a black, stable dispersion of MWCNTs in a PS solution. (B) TEM and (C) SEM micrographs of a spin-cast thin film of the MWCNT-PS composite. Inset: higher resolution TEM image, showing polymer-decorated MWCNTs. (D) TEM image of a perpendicularly microtomed film of the spin-cast MWCNT-PS composite prior to EHD patterning reveals round cross-sections of MWCNTs, indicating in-plane orientation of some of the MWCNTs in the as-spun film. 
Pristine MWCNTs with an average diameter of $10 \mathrm{~nm}$ were incorporated in PS with no additional chemical functionalisation prior to the dispersion process, thereby preserving their properties. A stable and homogeneous dispersion of MWNTs in PS was achieved using an established route described in the Experimental Section. A PS-MWCNT dispersion is shown in Fig. 5.2. Microscopy observations reveal that the individual MWCNTs are decorated by PS (Fig. 5.2B-C), sterically stabilizing the MWNCTs in solution and in the melt, aiding their efficient dispersion, while preserving their electronic and mechanical properties. [44, 99] This solution was then spin-cast onto a Si-wafer substrate, which served as an electrode in the EHD patterning process, to form a homogeneous nanocomposite film. It is important to note that at this stage no preferred orientation or alignment of MWCNTs was obtained, as confirmed by the random distribution of MWCNTs in the cross-sectional image of the composite (Fig. $5.2 \mathrm{D})$.

\subsubsection{EHD Patterning of the CNTs-Based Polymer Nanocom- posite}

These composite films were then subjected to EHD patterning. The physical mechanism of the EHD pattern formation process relies on an applied voltage across a polymer-air bilayer which exerts a destabilizing electrostatic pressure $p_{\text {el }}$ on the interface caused by interfacial polarization charges, [130]

$$
p_{\mathrm{el}}=-\varepsilon_{0} \varepsilon_{\mathrm{p}}\left(\varepsilon_{\mathrm{p}}-1\right) E_{\mathrm{p}}^{2}=-\varepsilon_{0} \varepsilon_{\mathrm{p}}\left(\varepsilon_{\mathrm{p}}-1\right) \frac{U^{2}}{\left[\varepsilon_{\mathrm{p}} d-\left(\varepsilon_{\mathrm{p}}-1\right) h\right]^{2}},
$$

where $\varepsilon_{0}$ is the dielectric permittivity of the vacuum, $\varepsilon_{\mathrm{p}}$ is the dielectric constant of the polymer, $h$ is local film thickness and $d$ is the capacitor plate spacing. $p_{\mathrm{el}}$ prevails over the counterbalancing surface tension of air-polymer interface $\gamma$. Using the lubrication approximation, this gives rise to the amplification of an instability with the characteristic wavelength

$$
\lambda=2 \pi \sqrt{\frac{2 \gamma}{\frac{-\partial p_{\mathrm{el}}}{\partial h}}}
$$

The amplification of fluctuation maxima drain the surrounding polymer film, laterally detaching the individual structures, and finally forming liquid cylindrical plugs between the two electrodes. The reorganized final morphology has a local hexagonal order caused by the repulsion of the polarized wave minima and maxima. The set-up is schematically shown in Fig. 5.3A, indicating the morphological reorganisation of the the MWCNT-PS composite. 


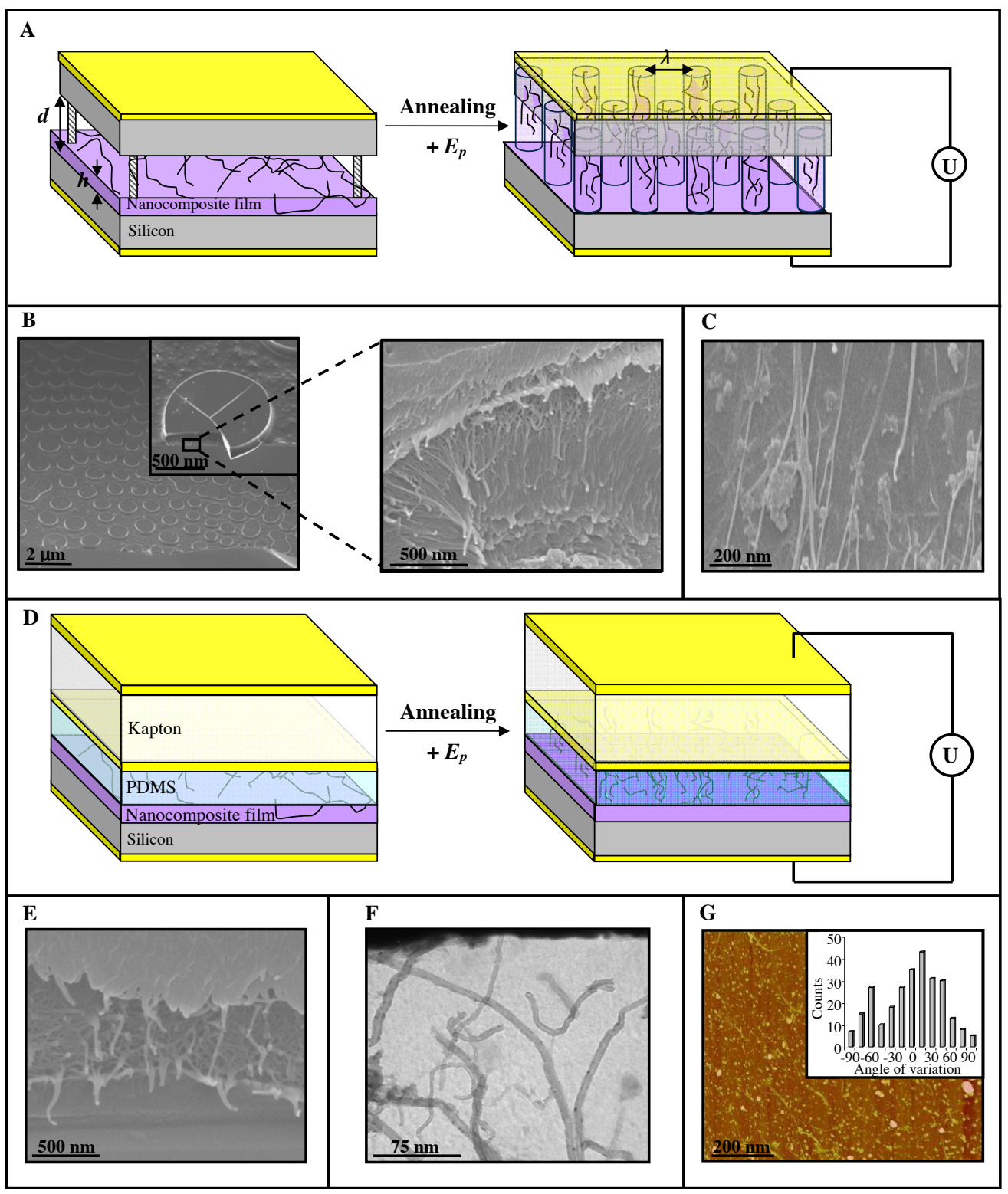

Figure 5.3: (A). Schematic representation of the representative experimental set-up and the overall pattern formation process in a laterally homogeneous electric field. Liquefying a thin nanocomposite film with randomly dispersed MWCNTs and subsequently applying a destabilising $E_{\mathrm{p}}$ causes an EHD instability with a characteristic wavelength $\lambda$. Amplification of the film undulations results in pillars with local hexagonal symmetry. (B). Low- and high magnification SEM micrographs of EHD patterned columns that contain vertically aligned MWCNTs. (C). Partial removal of the PS matrix provides an improved contrast of individual MWCNTs alignment as seen in the SEM cross section. (D). Experimental configuration for exposing nanocomposite films to an electric field, in the absence of an air gap. $4 \mathrm{kV}$ were applied across the Kapton, PDMS and nanocomposite film to give rise to similar electric field in the film as in (A). Cross-sections of films annealed in such a device: SEM (E), TEM (F) and AFM (G) images for an identical nanocomposite film annealed in an electric field. The histogram in (E) shows the relative MWCNT alignment with respect to electric field vector.

Films with a typical loading of $1.5 \mathrm{wt} \%$ of MWCNTs were first annealed at temperatures above the glass transition temperature of the polymer $\left(T_{\mathrm{g}} \approx 100^{\circ} \mathrm{C}\right)$, liquefying 
the material. Subsequently, a voltage of $120 \mathrm{~V}$ was applied across the electrodes, which gave rise to a laterally homogeneous electric field.

This electric field causes a film instability which leads to an array of columns that span the capacitor gap. The $1.3 \pm 0.3 \mu \mathrm{m}$ high vertical pillars with an average diameter of $1.0 \pm 0.2 \mu \mathrm{m}$ are shown in Fig. $5.3 \mathrm{~B}$ and C. The average inter-column spacing mirrors the characteristic wavelength $\lambda=1.2 \pm 0.3 \mu \mathrm{m}$. The mean spacing between formed columns corresponds to the wavelength predicted by Eq. (5.2). The inter-column spacing is determined by the field strength and the polymer surface tension and typically ranges from 0.5 to $30 \mu \mathrm{m}$ for values of $E_{\mathrm{p}}$ between $2 \times 10^{8}$ and $0.35 \times 10^{8}$ $\mathrm{Vm}^{-1}$ respectively, while the column diameter is a function of the initial film thickness and the plate spacing. The dimensions and lateral position of the generated patterns can be controlled by adjusting the different experimental parameters.

For instance, since liquid morphologies are organized according to the ratio of the plate spacing $d$ and the initial film thickness $h_{0}$, the variation of these two parameters determines the column height $(100-1000 \mathrm{~nm})$ and diameter $(800 \mathrm{~nm}-3 \mu \mathrm{m})$. On the other hand, the field strength and the polymer surface tension dictate the position of the formed structures by establishing the characteristic wavelength, i.e. the inter-pillar spacing.

While similar patterns were obtained for different MWCNT weight fractions, shorter instability wavelengths and hence inter-column spacings were observed for high MWCNT loading compared to the pure polymer, reflecting a higher destabilization pressure gradient $\partial p_{\mathrm{el}} / \partial h$. The origin of this effect is readily explained in terms of the dielectric properties of the composite. Similar to nanoparticles, [9] MWCNT loading increases the dielectric constant of the composite, thereby enhancing $E_{\mathrm{p}}$. [21]

Cross sectional examination of the pillars reveals an additional internal substructure comprised of vertically aligned MWCNTs along the axis of the columns (Fig. 5.3B) in the direction of the electric field lines. For better imaging, the samples were subjected to plasma-enhanced etching, which selectively removed a thin polymer layer from the surface, thereby further exposing the vertical MWCNTs caused by the faster etching rate of PS. A nearly right-angle orientation of MWCNTs relative to the capacitor electrodes is seen in Fig. 5.3C. Although short MWCNTs will align in the capacitor gap, they do not span the entire height of the formed columns. Long MWCNTs, on the other hand, align to form an interconnect between the two electrodes (Fig. 5.4). 


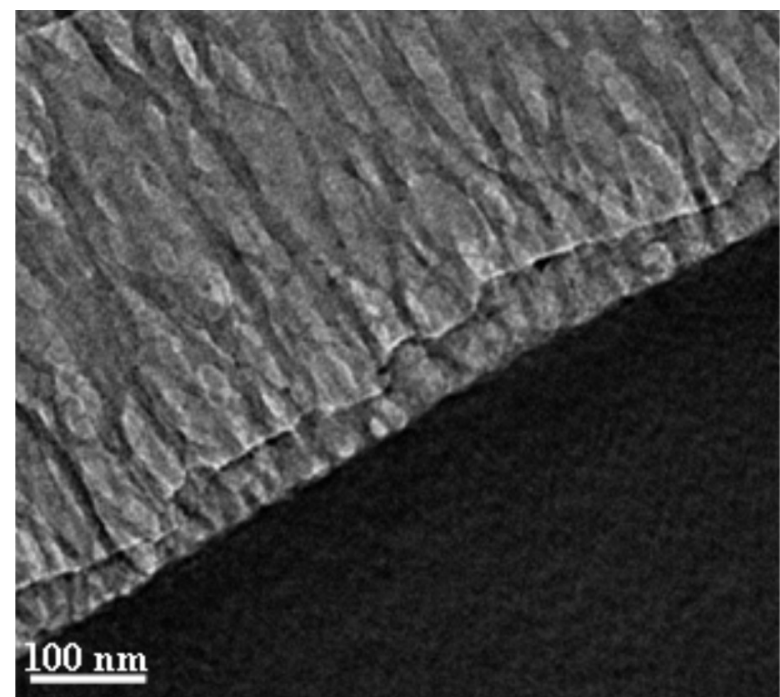

Figure 5.4: Ultrathin microtomed TEM image of the $450 \mathrm{~nm}$ height pillar with incorporated MWCNTs spanning the entire length of the formed pillar.

\subsubsection{Preferential MWCNT Alignment Within the EHD Gen- erated Structures}

A microtomed cross section parallel to the electric field direction examined by transmission electron microscopy (TEM) and atomic force microscopy (AFM) yields additional insight into the degree of MWCNT orientation. Figure 5.5 reveals that the vertically oriented MWCNTs are occasionally entangled. This is of importance with respect to the composite's electrical properties. MWCNTs, which span the entire EHD structure should have the highest conductivities for perfect vertical alignment, while shorter MWCNTs require some entanglements to ensure sufficient electrical contacts between the individual tubes that allow conduction via a percolating network. Note that the concentration of MWCNTs is higher at the surface of columns. This is most likely caused by high electric field gradients during the intermediate stage of the EHD instability: a sinusoidal undulation of a surface in a plate capacitor gives rise to a field concentration at the undulation maxima. [167] MWCNTs couple to this field gradient and experience a force in direction of the highest electric field, leading to a MWCNT accumulation in the region of the undulation that later makes contact with the upper capacitor plate, as seen in Fig. 5.3B and Fig. 5.5B. 


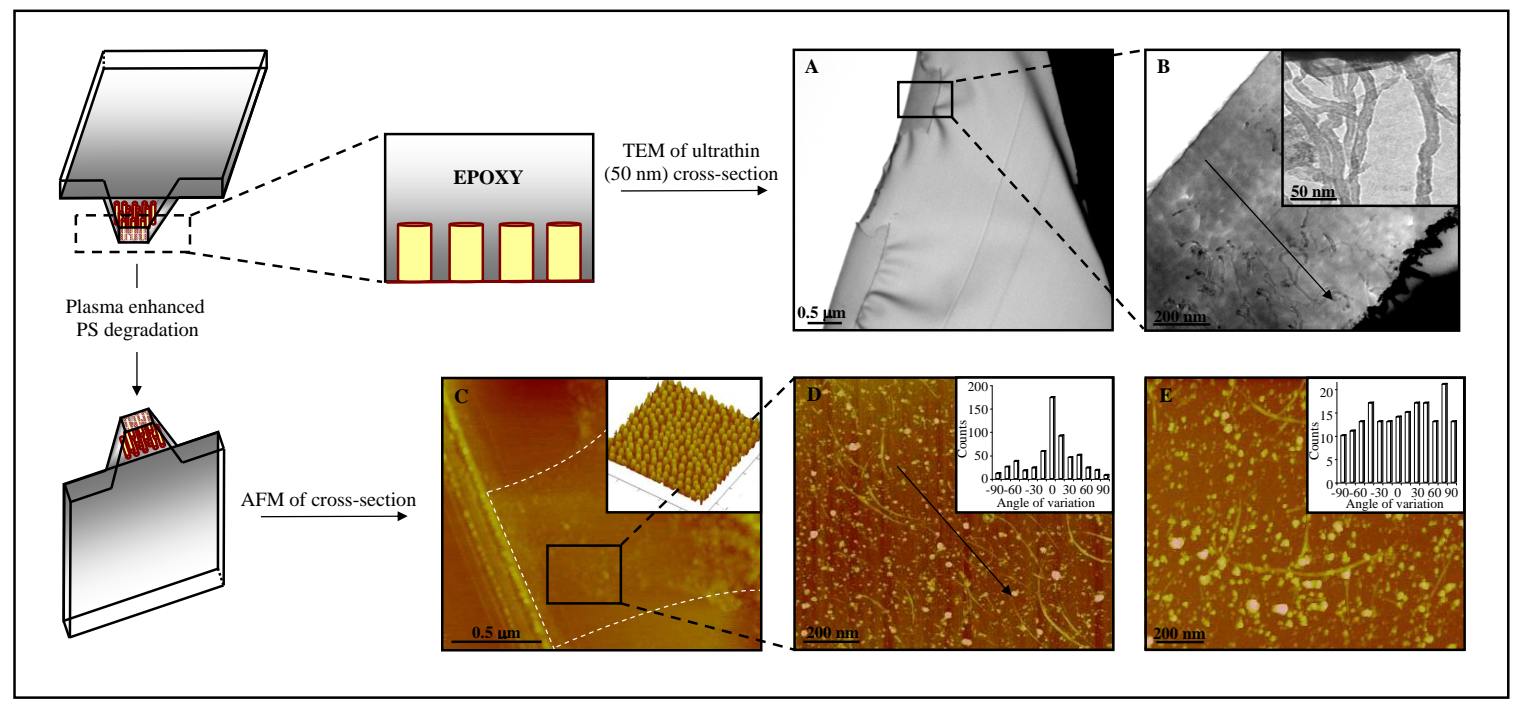

Figure 5.5: Schematic representation of sample analysis: following the embedding in an epoxy resin the lower electrode is removed, followed by microtoming ultrathin slices. (A,B) and (C,E) show TEM and AFM ultrathin sections parallel to to the direction of the electric field. Zooming into one of the columns of $(\mathrm{A})$ shows the nanotube orientation in direction of the pillar axis (B, arrow), with an increased concentration of aligned MWCNTs near the top electrode (B, inset). (C) AFM crosssectional view and $3 \mathrm{D}$ top-view showing the columnar pattern (inset). (D) High magnification AFM image of the cross-section of $(\mathrm{C})$, visualizing nanotube orientation in the EHD-formed pillar. (E). Cross-sectional AFM micrograph of the as cast nanocomposite film, revealing no preferred orientation of MWCNTs. The histograms show the degree of MWCNT alignment as an angle of variation from a cylinder axis in the patterned film (D, inset), indicated by the arrow, and from an arbitrary direction (E, inset) in the as-cast film.

To determine the average degree of MWCNT anisotropy inside EHD-formed columns, we have performed a quantitative analysis (see experimental section) of the TEM and AFM images in (Fig. 5.5B and D). Averaged alignment within an arbitrary axis (i.e., within \pm of the vertical axis or electric field lines)over the entire patterned area (approx. $200 \times 200 \mu \mathrm{m}^{2}$, averaged over 25 profiles), based on AFM and TEM images and statistical analysis (i.e, visual analysis within the human error) indicate that $80 \%$ of the MWCNTs were aligned within $\pm 20 \%$ of the electric field vector (Fig.5.5D, arrow) and $60 \%$ were within $\pm 15 \%$ of the director (Fig. $5.5 \mathrm{D}$, inset). This is in contrast to the as-cast nanocomposite films, where no preferred orientation was seen (Fig. 5.5E).

The preferential MWCNT alignment along the cylinder axis can be explained by two mechanisms. Firstly, the anisotropic MWCNT structure gives rise to a dipole moment that couples to the electric field, which exerts an aligning torque on the MWCNTs. Secondly, the EHD column formation gives rise to a lateral shear flow into the columns which may act to align the MWCNTs. 


\subsubsection{Mechanism Leading to the CNTs Anisotropy}

To address, which of these two proposed MWCNT alignment mechanisms is dominant, a second type of experiments was performed. Instead of leaving an air gap in the capacitor, the top electrode was brought into direct contact with the nanocomposite layer (Fig.5.3D). In this device geometry, the electric field does not cause any flow of the composite, but results only in an aligning torque on the MWCNTs.

The results of these experiments show a somewhat lower degree of nanotube alignment (Fig. 5.3E-G) of $45 \%$ and $37 \%$ within $\pm 20^{\circ}$ and $\pm 15^{\circ}$ of the electric field vector, respectively. This result indicates that the vertical orientation of the nanotubes in EHD patterned films arises from a combination of the electrostatic torque exerted on the nanotubes by the electric field and hydrodynamic convection forces and rotation by elongational component of the flow that aligns the MWCNTs during pattern formation.

\subsubsection{Electrical Properties of the Aligned CNT-Nanocomposites}

The good conductivity of MWCNTs raises the question, how MWCNT alignment in the insulating PS affects the conductivity across the composite film. To this end, the variation of electric resistivity was measured by a four probe method at room temperature as a function of MWCNT content, for as-cast films and for EHD patterned structures, as shown in Fig. 5.6. The introduction of $0.35 \mathrm{wt} \%$ of MWCNTs in an as-cast composite film lowered the resistivity by one order of magnitude. An additional increase in MWCNT content gradually decreased the resistivity by a further factor of 10 for a MWCNT content of $2 \mathrm{wt} \%$. While the conductivity of the EHD patterned sample measured along the cylinder axis (averaged over a large number of cylinders) is similar for MWCNT loadings above $1.3 \mathrm{wt} \%$, the results differ for small loadings from the unpatterned film. Interestingly, the averaged (appropriately normalized) axial resistivity of EHD patterned films is higher in the $0-1.3 \mathrm{wt} \%$ range, in which the resistivity varies only little, with a large drop between $1 \mathrm{wt} \%$ and $1.3 \mathrm{wt} \%$.

The difference in conductivity for MWCNT loading in the $0-1.3 \mathrm{wt} \%$ range can be understood in terms of a MWCNT percolation model. With a persistence length of $\sim 300 \mathrm{~nm},[79]$ non-aligned MWCNTs are entangled. Above the percolation threshold of $\sim 0.35 \mathrm{wt} \%$ MWCNT loading, continuous conducting pathways form across the composite film. The uniaxial alignment of the MWCNTs in the EHD-generated columns reduces the number of entanglement points per chain by straightening and aligning the MWCNTs. This increases the percolation threshold to a MWCNT loading of $\sim 1.3 \mathrm{wt} \%$. Although conductivities as high as $1 \mathrm{~S} / \mathrm{m}$ have been reported for composites with a MWCNT concentration of $5.5 \mathrm{wt} \%$, [183] this typically causes a short-circuit for the device used in the EHD experiments. 


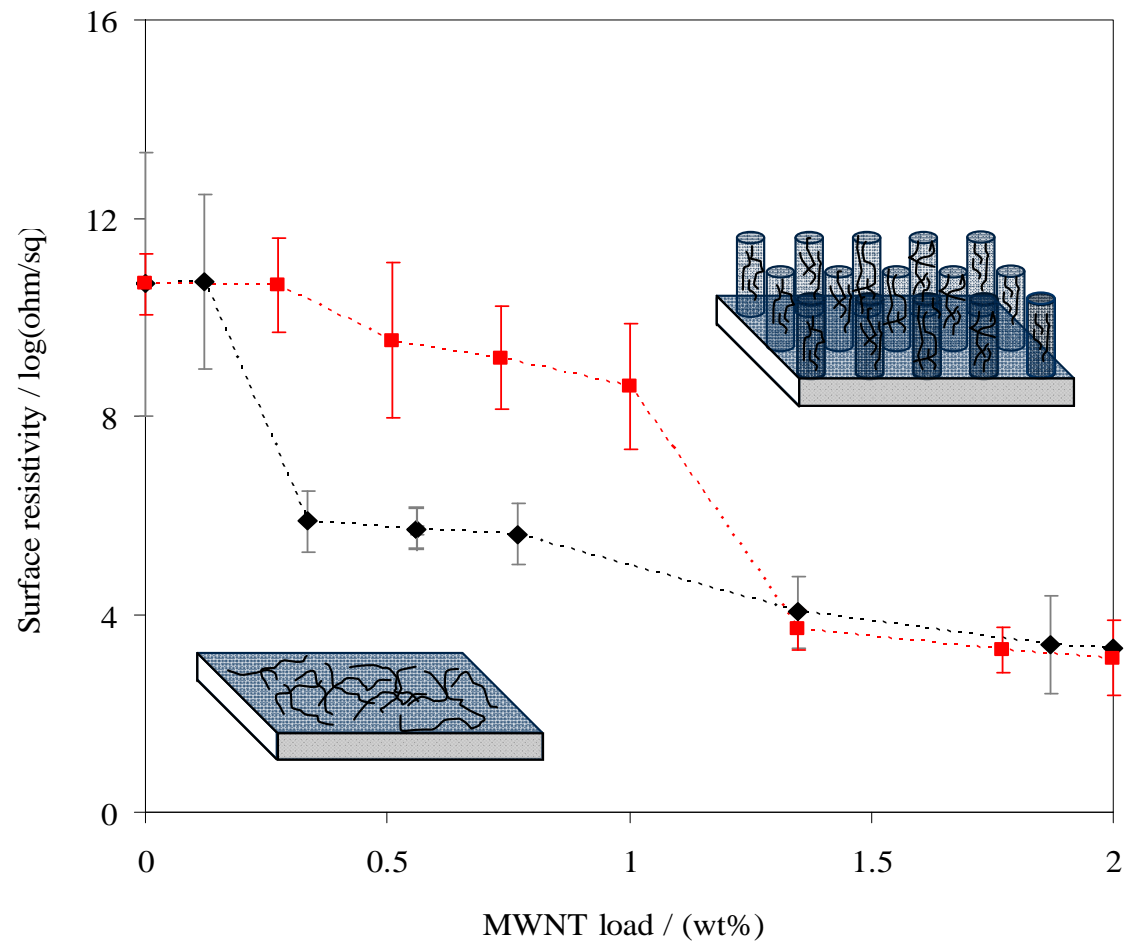

Figure 5.6: Resistivity of a spin-cast (diamonds) MWCNTs/PS composite film compared to an EHD-patterned nanocomposite (squares).

\subsubsection{Potential Uses of CNT-Brushes}

Finally, the embedded MWCNTs can be partially exposed upon process completion by the incomplete removal of the polymer from the top of the pillars by plasma etching. This results in a brush of vertically oriented partially free-standing nanotubes, which are supported by the remaining polymer matrix that prevents their collapse (Fig. 5.7). Alternatively, exposed vertically standing nanotubes can also be obtained without the polymer removal step, by using MWCNTs which are longer than the height of the formed columns (Fig. 5.7, inset). The creation of a well-defined supported CNT brush may be useful to establish an electrical circuit upon mechanically contacting plastic-based conducting composites. These hybrid vertically oriented structures therefore have potential applications for the development of flexible microelectronics, displays and biocatalytic assemblies, such as flexible field emitters, field emission devices (FEDs) or sensors. For example, a crucial aspect for an efficient FED is the distance between MWCNTs, which should be greater than their height to minimize the electric field screening effect. EHD-generated anisotropic patterned MWCNT arrays embedded in a polymer matrix not only directly lead to the suppression of the mutual screening, but also provide a way to control both the height and distance of columns decorated with aligned MWCNTs. Oriented CNTs with exposed ends exhibit 
an increased electrochemically accessible surface area. They may be used to generate well-defined electrical contacts and may provide a route for incorporating chemical functions by functionalization along their longtinudal tube axis. This is potentially useful for the development of rapid-response biochemical sensors which are selective for targeted chemical and biological molecules.

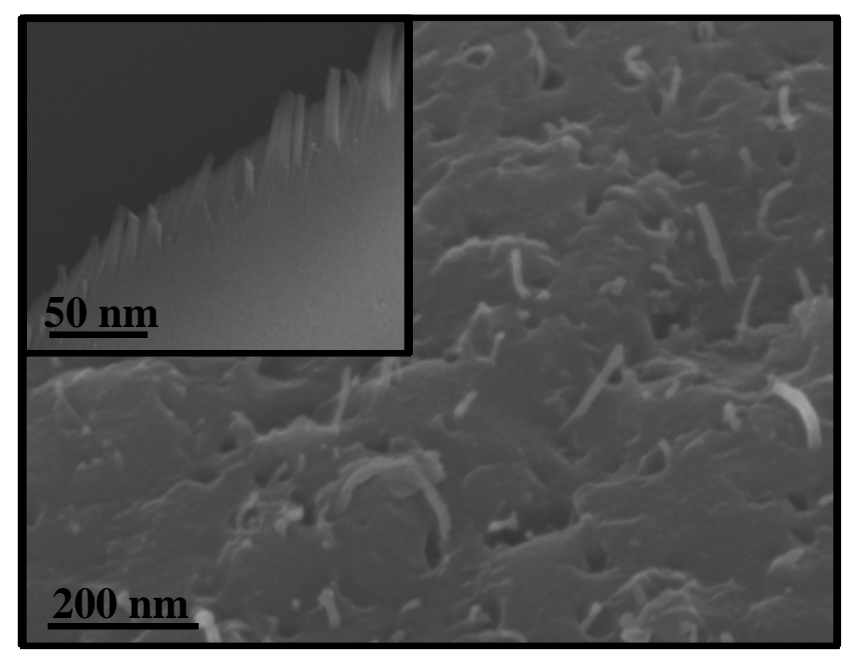

Figure 5.7: SEM top view of partially exposed vertical MWNTs embedded in a EHD-generated pillar. Using CNTs which are slightly longer than the pillar height results in similarly free standing nanotubes (inset).

\subsection{Conclusions}

In summary, we have introduced a straightforward technique for the hierarchical patterning of a polymer-carbon nanotube composite by means of an electric field. EHD patterning gives rise to a well-defined micron-scale columnar patterns with embedded MWCNTs which are uniaxially aligned parallel to the cylinder axis. With the use of a topographically patterned electrode [130] EHD lithography allows to control the lateral arrangement of the formed pattern as well as the alignment of the incorporated MWCNTs. Composite film conductivity as a function of MWCNT content shows a substantial increase in conductivity for a MWCNT loading of $\sim 0.3 \mathrm{wt}-\%$. Conductive pathways in percolating nanotube networks can be controlled by EHD patterning, shifting the percolation threshold by $\approx 1 \%$ for the field strength used in this experiment. Furthermore, the aligned MWCNTs can be exposed by selective polymer etching to form a MWCNT brush. The possibility of simultaneous structuring the nanocompsite and controlling the MWCNT alignment by EHD instabilities is an intriguing way to control the properties of the composite. Despite the reduction in conductivity upon MWCNT alignment, the creation of a MWCNT-brush is interesting to establish adhe- 
sive conducting electrical contacts, possibly paving the way for integration of nanotube based composites into functional devices. To this end, the incorporation and alignment of CNTs into semiconducting polymer (such as P3HT) will be an interesting future direction of study. 


\section{Hierarchical EHD Structures for Surface-Enhanced Raman Scattering}

Surface enhanced Raman scattering (SERS) is a well established spectroscopic technique that requires nanoscale metal structures to achieve high signals sensitivity. While most SERS substrates are manufactured by conventional lithographic methods, the development of a cost-effective approach to create nanostructured surfaces is much thought after in the SERS community. Here we establish a method to create controlled self-organized hierarchical nanostructures using electrohydrodynamic (HEHD) instabilities. The created structures are readily fine-tuned which is an important requirement for SERS. HEHD pattern formation enables the fabrication of multi-scale three-dimensional (3D) structured arrays as SERS-active platforms. Importantly, each of the HEHD patterned individual structural units yield a considerable SERS enhancement. This enables each single unit as an isolated sensor. Each of the formed structures can be effectively tailored to provide high SERS enhancement, while arising from different HEHD morphologies. The HEHD fabrication of sub-micrometer architectures is a straightforward and robust, providing an elegant route for high throughput biological and chemical sensing.

\subsection{Introduction}

Surface-enhanced Raman scattering (SERS) is an extremely sensitive molecular fingerprinting technique which can detect chemical substances down to the single molecule level $[72,100]$ with important applications in forensics, healthcare and diagnostics. SERS is based on the electromagnetic field enhancement via localized optical fields (plasmons) on nanostructured metallic structures. [10, 102] Localized surface plasmon resonance (LSPR) can be tuned by manipulation of the surface architecture at the submicron level, giving rise to the Raman signal enhancement SERS is known for. Hence, controlled surface fabrication techniques underpin the emergence of next-generation plasmon-active devices such as those used in SERS. The development of robust, cost- 
effective and simple technique therefore, enables the design and construction of wellcontrolled large area surface structures with mesoscopic lengths scales.

The vast majority of earlier synthetic routes to generate SERS structures with suitable reproducibility are based on conventional lithographic techniques. [110] These are not only expensive and time consuming but are also often cumbersome and require precise integration of multi-step processes, thus, limiting the scalability of the resulting structures. For instance, while photolithography [94] is a flexible method for generating optical patterns, the technique is limited by the optical diffraction limit and is essentially two-dimensional (2D), meaning that many steps must be iterated to create a 3D structure. Electron beam lithography is an alternative approach that uses a scanning beam of electrons [63] to write sub-micrometer structures into a resist that can subsequently be transferred to the substrate material, often by etching. The preparation of such patterns not only requires sequential writing steps, but is also vulnerable to beam drift or instability which may occur during the long exposure times. Limited resolution, poor mechanical stability of the mould, and pattern distortion are the limiting factors of microlithography and other imprinting techniques. [23, 113] Alternative routes for multiscale structures include self-assembly [181] or bio-mimetic processes, but, the precise control of features and prevention of defects remains a challenge, particularly in the sub-micron regime. The limits of the above-mentioned top-down approaches and self-assembly based bottom-up approaches highlight the need for more reliable, costeffective and straightforward patterning method to develop tunable 3D structures.

Here, we report an approach that addresses some of the problems described above, and enables the formation of hierarchical 3D structures with three independent, characteristic lateral dimensions. Hierarchical electrohydrodynamic (HEHD) pattern formation harnesses a sequential instability in multilayer thin films induced by an electric field $E_{\mathrm{f}}$ to guide the layer material into design structures, allowing different materials to be patterned in a one-step procedure. HEHD offers a great variability of the structural features by the independent alteration of several parameters. Hierarchical polymer structures covered by a plasmon-active-metal (in this case gold) effectively enhance the EM field, in the fine multiscale architecture.

\subsection{Experimental}

PVAC, PVA (Sigma-Aldrich) and EC (Fluka), were used without further purification. Highly polished $p$-doped silicon wafers, with (100) crystal orientation (Wafernet GmbH, Eching, Germany) were used as substrates.

Prior to spin-coating, the substrates were cleaned in a 'Piranha' solution consisting of $3: 1 \mathrm{H}_{2} \mathrm{SO}_{4}(98 \%): \mathrm{H}_{2} \mathrm{O}_{2}(30 \%)$, followed by thorough rinsing with deionised water 
and drying under nitrogen. The ITO-coated glass slides were cleaned by scrubbing in soap water at $75^{\circ} \mathrm{C}$ and washing in an ultrasonic bath with acetone and isopropanol, followed by irradiation for $20-30 \mathrm{~min}$ in a UV-ozone cleaner. Immediately before device assembly, all substrates and electrodes were subjected to snowjet cleaning.

First, a thin film of EC $\left(\gamma=28.5 \mathrm{mNm}^{-1}, T_{\mathrm{g}}=130^{\circ} \mathrm{C}, \eta=0.084 \mathrm{~Pa} \cdot \mathrm{s}\right), 150 \mathrm{~nm}$ in thickness, was spin-coated onto a freshly cleaned Si substrate from toluene. A $110 \mathrm{~nm}$ layer of PVA $\left(\gamma=50 \mathrm{mNm}^{-1}, T_{\mathrm{g}}=85^{\circ} \mathrm{C},=\eta=0.073 \mathrm{~Pa} \cdot s\right)$ was then spin-coated directly on top of the EC film from water solution to form an EC/PVA bilayer. Water is a nonsolvent for EC. For a third layer film PVAc $\left(\gamma=57.8 \mathrm{mNm}^{-1}, T_{\mathrm{g}}=30^{\circ} \mathrm{C}\right.$, $\eta=0.225 \mathrm{~Pa} \cdot s)$ with thickness of $100 \mathrm{~nm}$ was spin cast from toluene and further was floated onto a pool of deionized water and then transferred onto the PVA layer to form an EC/PVA/PVAc trilayer. The sample was subsequently placed into a vacuum oven to remove the residual water.

A capacitor set-up was assembled using the trilayer covered substrate as one of the electrodes. Facing it, second silicon wafer was mounted leaving a thin air gap. The overall electrode spacing, $d$ was adjusted by using lithographically prepared spacers or silicon oxide colloids as spacers. Backsides of the both electrodes were coated with a thin gold layer $(h=100 \mathrm{~nm})$ followed by electrically contacting them using silver paste (Electrodag $1415 \mathrm{M}$ ). The films were sequentially liquefied by thermal annealing, raising temperature above the $T_{\mathrm{g}}$ of each the resist, and a voltage of $80 \mathrm{~V}$ was applied to the capacitor device. The experiments were terminated by quenching the assembly to room temperature before removing the electric field. PVA was removed by dissolution in distilled water which is a non-solvent for both the EC and PVAc. Cycloheptane or cyclopentane were used to dissove the central PVAc column without removing the EC rim.

The evolving patterns were observed with a standard optical microscope (Olympus BX 40) and an inverted reflective optical microscope (Olympus GX61) operated in clean-room conditions. Digital cameras connected to both microscopes were read out by imaging software (Carl Zeiss Visiocam). A NanoScope IV Multimode Dimension 3100 atomic force microscope (AFM) was used to quantitatively determine the sample topography. Height and phase images were analyzed with the Nanoscope software (Digital Instruments). The AFM measurements yielded the film thickness $h$, the plate spacing $d$, the characteristic wavelength $\lambda$, and the width and height (i.e., aspect ratios) of the generated patterns. Optical and AFM micrographs showed the lateral distribution of the patterned polymers on the substrate. The scanning electron microscopy (SEM) measurements were performed using a LEO ULTRA 55 SEM including a Schotthy emitter $(\mathrm{ZrO} / \mathrm{W}$ cathode) at acceleration voltages of $5.0-10.0 \mathrm{kV}$ with a lateral resolution of $2-5 \mathrm{~nm}$. 
SERS measurements were carried out with an InVia Renishaw Raman Microscope System equipped with $633 \mathrm{~nm}$ and $785 \mathrm{~nm}$ lasers. The spectra were typically acquired with a $10 \mathrm{~s}$ exposure time and a laser power of $3 \mathrm{~mW}$ and $10 \mathrm{~mW}$ at the sample at $633 \mathrm{~nm}$ and $785 \mathrm{~nm}$, respectively. SERS maps were acquired in Streamline mode (line scan) with $20 \mathrm{~s}$ exposure time and $6 \mathrm{~mW}$ and $100 \mathrm{~mW}$ power at $633 \mathrm{~nm}$ and $785 \mathrm{~nm}$, respectively. A $50 \times$ objective with a numerical aperture of 0.75 was used for all measurements. Optical measurements were carried out with an Olympus BX51 microscope equipped with an incoherent white light (halogen) source and an optical fibre coupled to a QE65000 Ocean Optics spectrometer. The spectra were normalized with respect to those recorded on flat gold.

\subsection{Results and Discussion}

\subsubsection{Principles of Bespoke HEHD Pattern Formation Process}

The EHD patterning process is very well understood for single liquid layers, and polymer bilayers. [85, 86, 107, 108, 131, 169] Here, we have extended this principle to polymer trilayers in our HEHD approach. The structure formation in a multilayer film can be controlled by carefully designing the layer sequence. In a $\operatorname{polymer}\left(P_{1}\right)$ - $\operatorname{polymer}\left(P_{2}\right)$ polymer $\left(P_{3}\right)$-air trilayer (Fig. 6.1A), a careful selection of polymers prevents uncontrolled dewetting of the multilayer allowing control over sequential destabilization of the trilayer assembly layer by layer. To this end, the trilayer is assembled according to the glass transition temperature $T_{\mathrm{g}}$, of the individual layers, starting from the highest $T_{\mathrm{g}}$ for the bottom film and the lowest $T_{\mathrm{g}}$ for the upper layer (i.e., $T_{\mathrm{g} 1}>T_{\mathrm{g} 2}>T_{\mathrm{g} 3}$ ). This way, each layer can be liquefied individually while preserving the underlying layer in a stable state. The resulting hierarchical architectures are an outcome of sequential electrostatic destabilization. Annealing at $T_{\mathrm{g} 3}<\mathrm{T}<T_{\mathrm{g} 2}$ with a constantly applied $E_{\mathrm{f}}$ results in the destabilization of the upper layer, forming pillars which span from the film surface to the upper electrode (Fig. 6.1A-B). The primary pillar formation of $P_{3}$ paves the way for the deformation of the intermediate layer at the $P_{2}-P_{3}$ contact line, where electrostatic forces are the highest. Once the primary columns formed, raising the temperature to $T_{\mathrm{g} 2}<\mathrm{T}<T_{\mathrm{g} 3}$ while continuously applying $E_{\mathrm{f}}$, the destabilizing EHD pressure drives a flow of $P_{2}$ to the upper electrode along the outside of the $P_{3}$ column (Fig. 6.1B-C). This generates the $P_{2}$ mantle surrounding the $P_{3}$ primary column. Upon completion of the secondary instability, $T_{\mathrm{g}}$ is further raised to $\mathrm{T}>T_{\mathrm{g} 3}$ generating a third instability in $P_{1}$. Again,the liquified material of $P_{1}$ flows upwards around the secondary $P_{2}$ cylinder (Fig. 6.1C-D). Wetting properties of the three materials come also into play once the composite columns have formed. Therefore, organizing 
the polymers according to the increasing surface tension (i.e., $\gamma_{1}<\gamma_{2}<\gamma_{3}$ ) stabilizes the final coaxial structures. Sequencing of the polymer layers is also chosen according to the increasing viscosity (e.g., $\eta_{1}<\eta_{2}<\eta_{3}$ ) to ensure short times for the formation of the patterns.
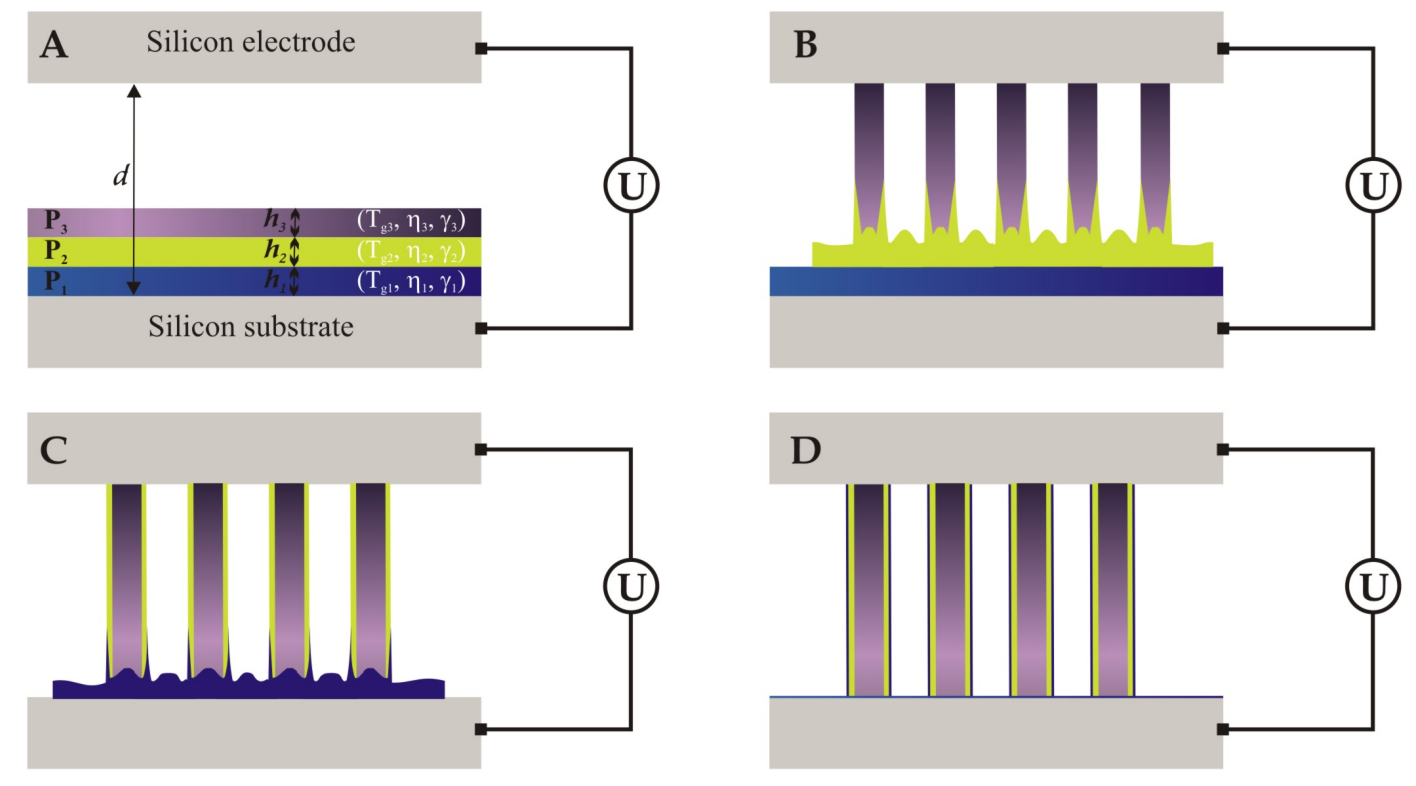

Figure 6.1: Mechanism of the HEHD structure formation process.(A). The initially assembled polymer trilayer is destabilized by applying a voltage, $U$. (B). The primary instability yields pillars which span the capacitor gap. The secondary instability arises from the deformation of the intermediate layer, $P_{2}$ occurring at the $P_{2}-P_{3}$ contact line during the primary pillars formation process. The amplification of the secondary instability guides the intermediate layer upwards forming a surrounding shell outside the primary column. (C). Similarly, a third instability takes place at the bottom film and the liquified $P_{1}$ polymer is drawn upwards by the electrohydrodynamic torque while forming an additional mantle around the bilayer pillar. (D). The final configuration consists of a core-shell $1-$ shell $_{2}$ multiplex structure comprised of a primary $P_{3}$ pillar, coated by additional concentric $P_{2}$ cylinder and the outer $P_{1}$ mantle.

A schematic representation of the method is shown in Fig. 6.2, in which multiscale-patterns are created by HEHD patterning. Three materials: ethyl cellulose (EC), polyvinyl alcohol (PVA) and polyvinyl acetate (PVAc) were chosen to be sequentially patterned as they exhibit the required parameters $\gamma_{\mathrm{EC}}<\gamma_{\mathrm{PVA}}<\gamma_{\mathrm{PVAc}}, T_{\mathrm{EC}}>T_{\mathrm{PVA}}>T_{\mathrm{PVAc}}$ and $\left.\eta_{\mathrm{EC}}<\eta_{\mathrm{PVA}} \approx \eta_{\mathrm{PVAc}}\right)$. Slight wedge geometry was created deliberately yielding spacing variation $d$ from $100 \mathrm{~nm}$ to $1 \mu \mathrm{m}$ across the $1 \mathrm{~cm}$ sample. Consequently, the interfaces are destabilized at different times leading to a sequential lateral redistribution of all three materials. Due to the wedge geometry of the sample, different stages of instability can be obtained on the same sample. Initially, the film develops lowamplitude undulations (Fig. 6.2B, right hand side), which are further amplified and 

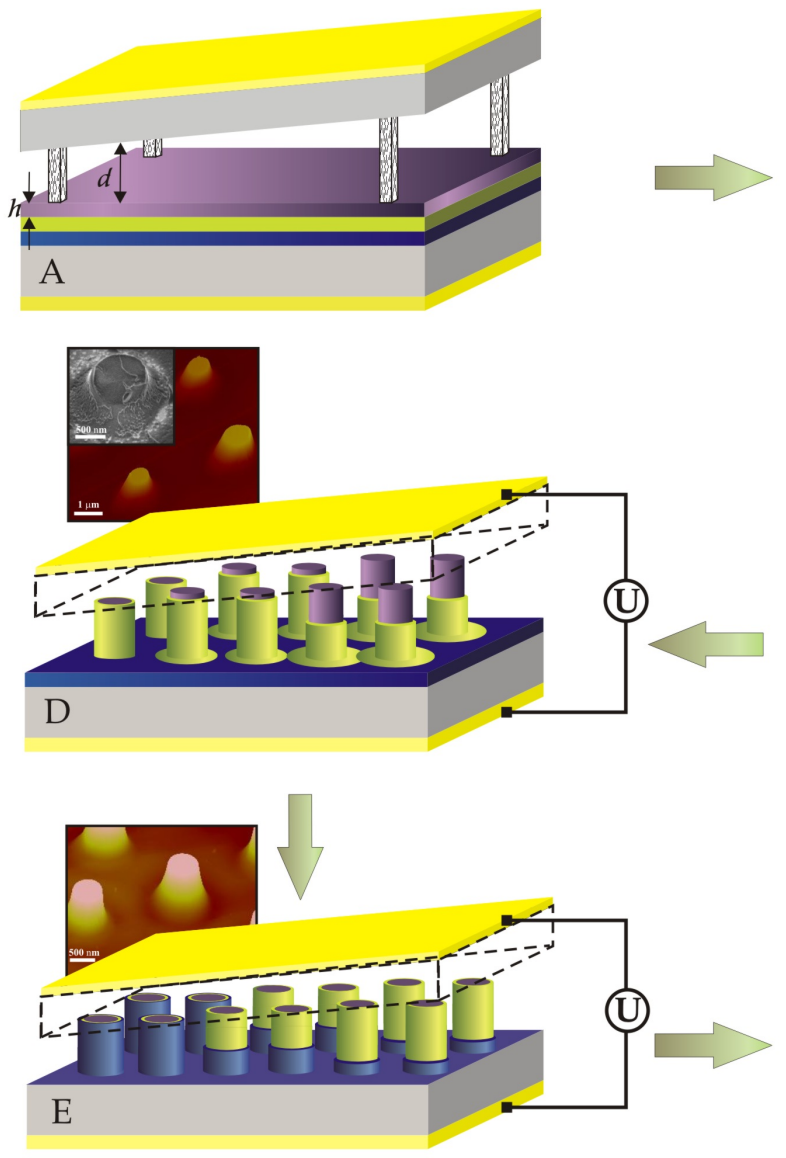
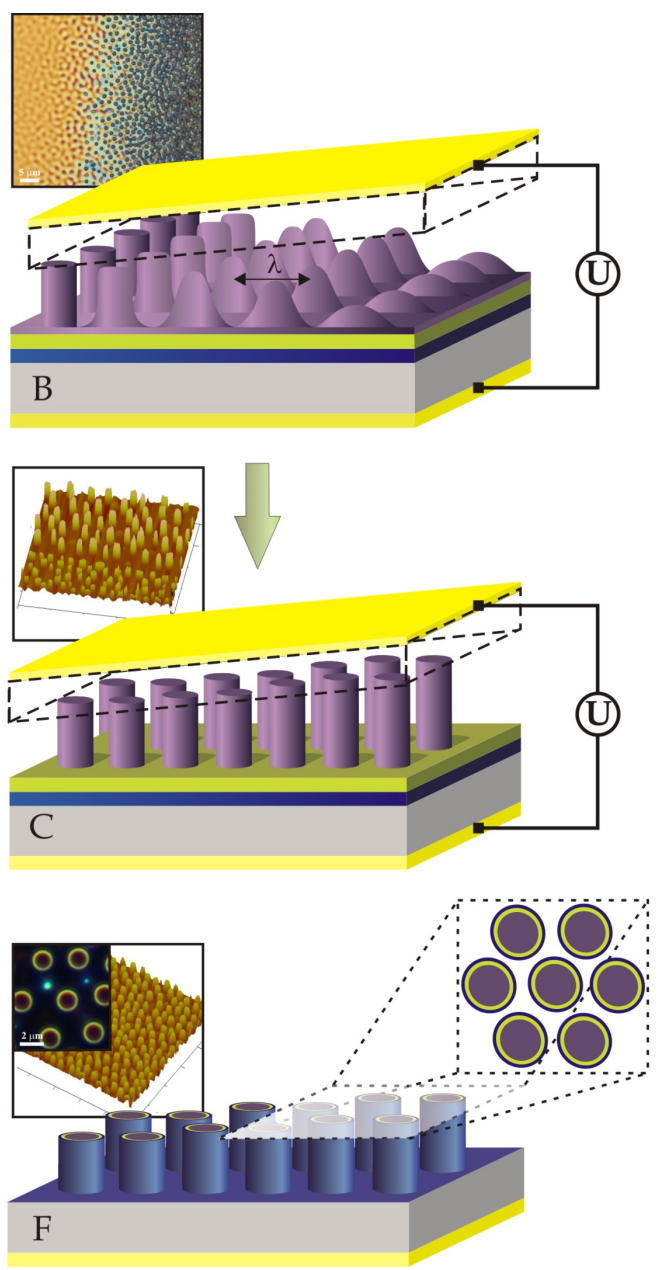

Figure 6.2: Schematic representation of the experimental set-up and sequential steps of the HEHD patterning process. (A). Three thin layers of EC, PVA and PVAc deposited on top of each other in the capacitor device are sequentially liquefied by annealing above $T_{\mathrm{g}}$ of each resist. (B) Amplification of a capillary surface instability in the upper layer (PVAc) with a lowest $T_{\mathrm{g}}$ (and highest $\gamma$ ) is triggered by applying a voltage $U$. With time the instability causes the formation of liquid bridges between the two electrodes. An instability front sweeps laterally across the sample with wedge geometry and different stages of the pattern formation can be observed (B, inset) on the same sample, starting from instability with characteristic wavelength through amplified undulations to final columns that span the capacitor gap seen in (C). The wedge geometry yields pillars which exhibit a range of aspect ratios as shown in the AFM image (C, inset). (D). Upon completion of the HEHD patterning of the upper layer, further annealing above the $T_{\mathrm{g}}$ of the intermediate film (PVA) results in secondary HEHD instability generating gradually growing mantles surround the primary PVAc columns. (E). Third, and final instability evolves upon annealing the bottom (EC) film above its softening temperature resulting in hierarchical structures with a cylindrical PVAc core, surrounded by a mantle of PVA and a secondary shell of EC seen in (F). Three-dimensional AFM image reveals well organized pillars consisting of three concentric cylinders as shown in optical micrograph (F, inset: left hand side) and by schematic zoom-in top view ( $\mathrm{F}$, inset, right hand side).

eventually span the two electrodes forming an array of liquid pillars with local (and sometimes global) hexagonal symmetry (Fig. 6.2B, left). The intermediate stage in Fig. $6.2 \mathrm{~B}$ is the consequence of the variation in the inter-electrode spacing, resulting in 
a range of stages of the instability (right: undulations; left: columns) which eventually form cylindrical columns (Fig. 6.2C). The structures formed at this stage have a continuous variation of aspect ratio along the wedge shaped cell. A representative AFM image revealing the lateral variation in pillar aspect ratio is shown in Figure $6.2 \mathrm{C}$, inset. These primary pillar structures therefore lend themselves to study and optimize the best geometry for SERS substrates in a combinatorial manner.

HEHD patterning produces hierarchy of length scales of the generated structures. The size-scales of hierarchical morphologies can be controlled via tuning the experimental parameters. Since there are three interfaces that may become unstable, various structures can emerge with the morphologies depending on the ratio of the thicknesses. In addition, due to the wedge geometry, the instability does not develop everywhere in the capacitor at the same time but swing laterally across the sample. This property can be exploited to provide an additional degree of structures tuning. Termination of the process at any given time enables achieving structures with tailored dimensions and aspect ratios. This is of an invaluable importance for optimizing structures for SERS, since the plasmon resonances can be in this way tuned around the excitation laser frequencies.

\subsubsection{Variation of SERS Surface Enhancement and Reflectance with Aspect Ratios of Primary Pillars}

In order to evaluate the SERS performance of HEHD patterned structures shown in Fig. 6.2C, they were coated with $20 \mathrm{~nm}$ thick gold layer followed by an adsorption of a benzenethiol monolayer. The SERS spectra recorded for these pillar-like single column structures are shown in Fig. 6.3A. In addition to a global experiment SERS mapping was carried out for individual pillars. Representative SERS map overlaid on its corresponding optical image is shown in Fig. 6.3B. The SERS signal (red) comes directly and only from the EHD pillars. As apparent from Fig. 6.3A the electrode wedge geometry results in a continuous variation of the pillar aspect ratio. Figure 6.3B shows the variation of the SERS enhancement as a function of the pillar aspect ratio. Single columns of aspect ratio 0.79 gave a nearly 30 times enhanced SERS signal compared to a flat film, at a laser excitation of $633 \mathrm{~nm}$. Similar trend was obtained in the near-infrared $(785 \mathrm{~nm})$ with high signals obtained for highest aspect ratios. Note that the SERS of Fig. 6.3A arises practically from a single column. Unlike most other submicron SERS structures a change in the inter-column spacing does not modify the SERS signal. Individual pillars of HEHD patterned therefore function as an isolated detection centre, thus, rendering these patterned substrates as platforms for multiplex SERS of different chemical species. 

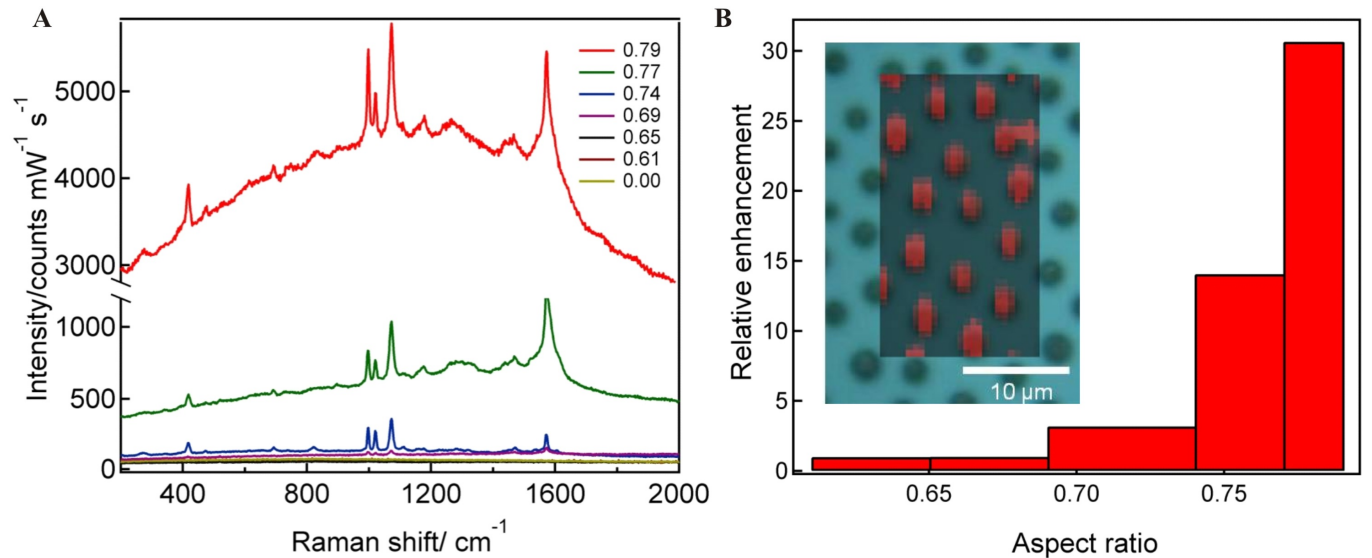

Figure 6.3: SERS surface enchantment versus aspect ratio. (A). SERS spectra of benzenethiol recorded from substrates with pillars of various aspect ratios. (B) High aspect ratio structures show high SERS signals. The inset shows a SERS map overlayed over the optical image of a patterned area. The SERS signals for the $1070 \mathrm{~cm}^{-1}$ peak is in register to the column locations.

Figure 6.4 shows the spectrally resolved SERS reflectance across a sample consisting of columns with laterally varying aspect ratios (inset). Larger strength LSPR result from higher absorption of the resonant electromagnetic radiation, and consequently, generation of considerably larger electromagnetic fields at the surface of the roughness feature. The reflectance spectrum shows increasing absorption at the pump excitation of $633 \mathrm{~nm}$ and $785 \mathrm{~nm}$ (dashed lines) with increasing pillar aspect ratio. The inset shows this variation at $785 \mathrm{~nm}$, with a nearly $30 \%$ increase in absorption for a change in aspect ratio from 0.6 to 0.8. This absorption arises from plasmonic resonances which are tuned by the pillar geometry. The relative SERS enhancement of Fig. 6.3 is correlated with the increased strength of plasmonic absorption of Fig. 6.4. The tuneability of the aspect ratio and its effect on plasmonic absorption and SERS signal allows the optimization of substrates for different laser excitations.

\subsubsection{Generation of Hierarchical EHD Architectures}

Hierarchical structures can be formed by enabling secondary instabilities of the PVA layer, once the PVAc columns have fully formed (Fig. 6.2C). The electrostatic pressure gradient at the surface of the primary column drives the flow of the underlying layer, in our case dielectric PVA, upwards along the PVAc pillars, forming a thin enclosure shell (c.a. diameter of $170 \mathrm{~nm}$ ) around the inner cylindrical column (c.a. diameter of $750 \mathrm{~nm}$ ) as the core. Again, intermediate stages with of PVA shell heights can be obtained because the instability front sweeps laterally across the sample caused by the wedge geometry of the electrodes (Fig. 6.2D). Similarly a third instability can be triggered by 


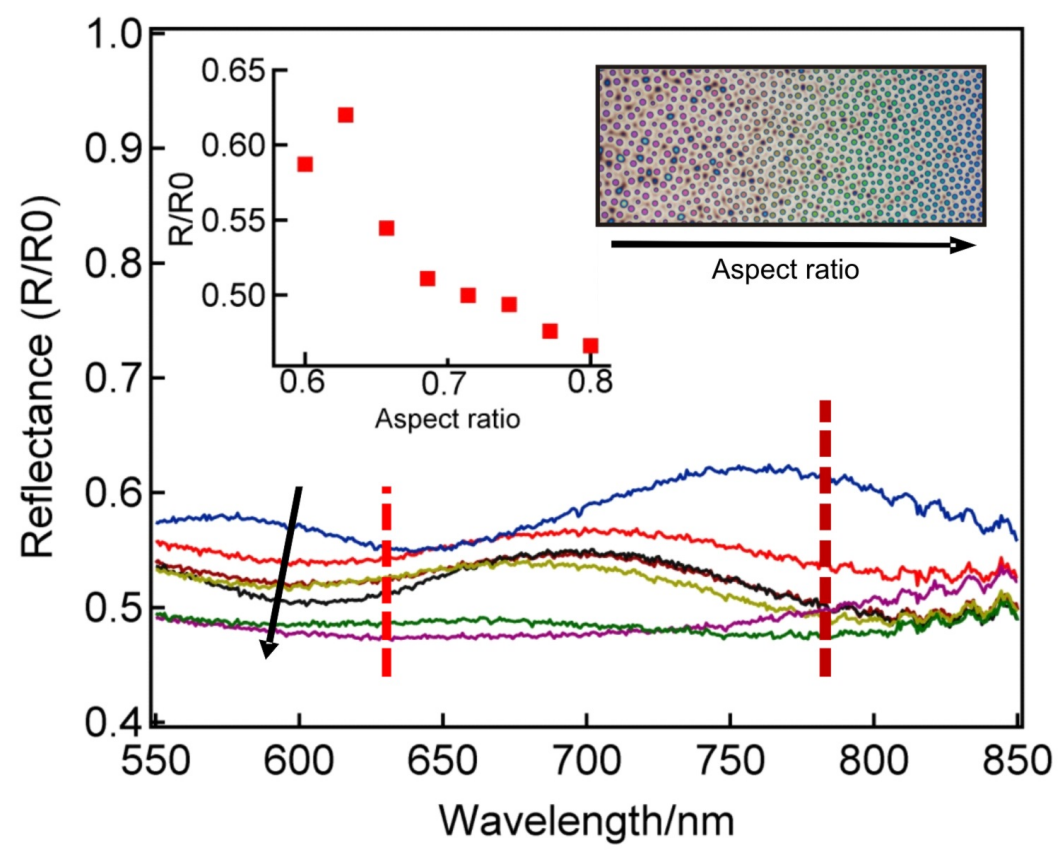

Figure 6.4: Reflectance spectra (acquired at the same locations as in Fig. 6.3), corresponding to different aspect ratios of the EHD patterned structures. The inset shows the variation in reflectance (where $R_{0}$ is the reflectance from the flat gold surface) at $785 \mathrm{~nm}$. The inset shows a large area optical image of the pillar array. The color variation across the sample arises from the change in aspect ratio. The reflectance expressed as a decreasing ratio between the structured to unstructured gold substrates (i.e., increase in the ratio of the absorption) with increasing pillar aspect ratio arises from plasmonic resonances, indicating increased strength of LSPR and therefore, higher SERS enhancement.

liquefying the lowest layer (Fig. 6.2E) following the completion of the coaxial pattern stemming from the upper bilayer. Using EC as the third underlying layer, hierarchical structures were fabricated which have a cylindrical PVAc core, surrounded by a mantle of PVA and a second shell of EC. The overall diameter of a trilayer concentric pillar was $1.09 \pm 0.10 \mu \mathrm{m}$, with an average height of $771 \pm 8.00 \mathrm{~nm}$.

Once the hierarchical pillars have fully formed, (Fig. 6.5A) a variety of multiscale structures can be realized by simple subsequent washings procedures. Central pillars were created by washing out the intermediate PVA shell with a non-solvent for the PVAc and EC. This resulted in a primary column of PVAc with a typical diameter of $750 \pm 21 \mathrm{~nm}$, surrounded by a thin circular rim of EC with a wall thickness of only $87 \pm 13 \mathrm{~nm}$ (Fig. 6.5B). The gap width between the pillar and the rim was $130 \pm 19 \mathrm{~nm}$. The thickness of a thin EC layer left covering the bottom electrode and the amount of EC material driven into the rim depends on the interplay of wetting and electrostatic driving force. Because of the relatively high surface energy of the substrate, the height of the EC rim is typically lower than that of the central cylinder (Fig. 6.5B, inset). An additional washing step removing the central column leaves behind volcano rims of EC 


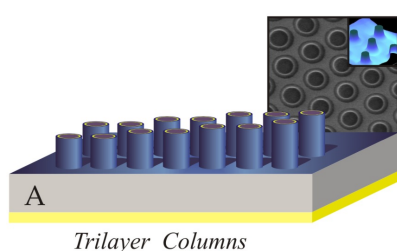

Trilayer Columns

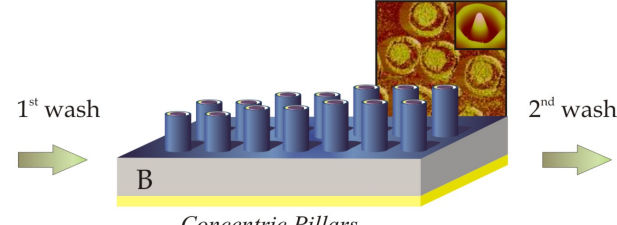

Concentric Pillars

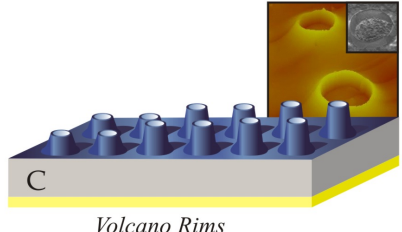

Volcano Rims

Figure 6.5: Generation of hierarchical architectures. Schematic and corresponding AFM and SEM images (insets) of sequential washing steps of the trilayered coaxial pillars in (A), resulting in concentric structures comprised of central PVAc pillar surrounded by a thin EC rim in (B) and, in volcano rims of EC $(\mathrm{C})$. Hierarchical structures with lateral dimensions ranging from $1 \mu \mathrm{m}$-down to below-100 nm were formed using the HEHD patterning technique.

(Fig. 6.5C, inset) with a width of only $87 \mathrm{~nm}$, considerably smaller than the width of the primary PVAc column. Hence, HEHD patterning enables the generation of design sub-100 $\mathrm{nm}$ features that are arranged in a lateral array. Again, the electrode wedge geometry generates a range of structural rim parameters.

\subsubsection{HEHD Formed Patterns as Advanced Substrates for SERS}

The detailed control over the column morphology and their aspect ratio offered by HEHD patterning provides advanced substrates for SERS. To this end the polymer hierarchical structures were coated with $20 \mathrm{~nm}$ of gold and covered by an immobilized monolayer of benzenethiol molecules. Depending of the chosen morphology, substantial variations in the SERS signals were found (Fig. 6.6A). To point out that, while the absolute enhancement referrers to the Raman signal from structured surface with reference to the bulk solution, the relative enhancement is used to compare between the signals from different structures on the substrate. The concentric pillar-rim structure shows a 25-fold compared to the simple pillars and a nearly 200 times fold enhancement over the volcano ring structure at a wavelength of $785 \mathrm{~nm}$ (Fig. 6.6B). Difference in SERS signals at $633 \mathrm{~nm}$ is less dramatic with the simple pillars and the concentric pillar-rim structure showing similar signals and the volcano structures showing an approx. 5 times lower signal. The absolute enhancement for the concentric pillar-rim SERS substrate was $\sim 10^{7}$ at $785 \mathrm{~nm}$ and $1.5 \times 10^{6}$ at $633 \mathrm{~nm}$. An enhancement of $1.2 \times 10^{6}$ was measured for simple columns at $633 \mathrm{~nm}$. These values compare very favorably with commercial SERS substrates, typically reporting an enhancement of $\sim 10^{6}$. [5] Note that these enhancement factors are normalized by illuminated area (spot size) while the SERS activity is localized at the surface structure and therefore stems only from a small faction of the sample area. 
A

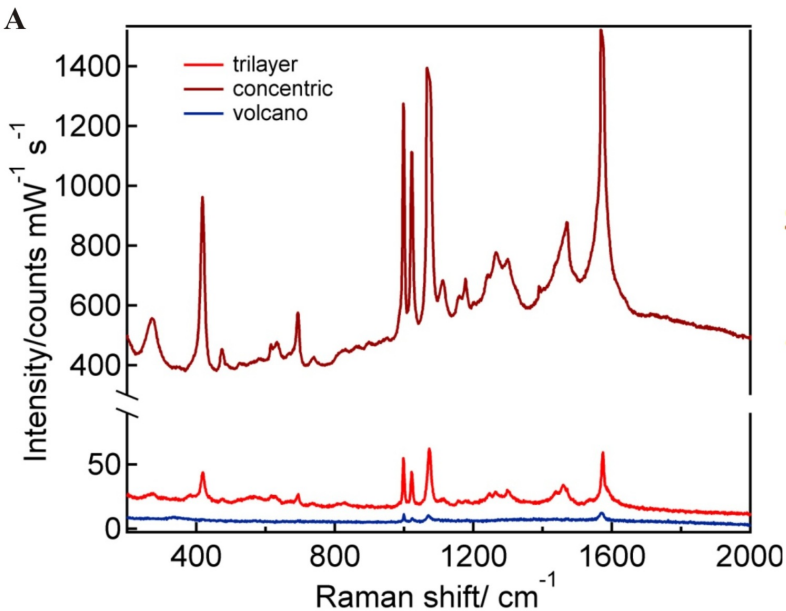

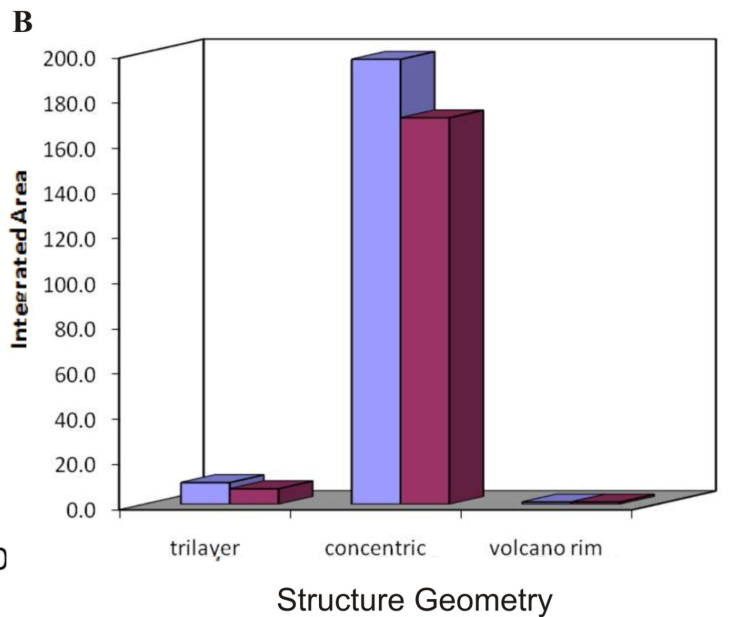

Figure 6.6: (A). SERS of benzenethiol recorded on three different surface structures generated by HEHD. The highest signal was obtained for the concentric pillar-rim structure. (B) The relative enhancement is shown for the $1000 \mathrm{~cm}^{-1}$ and $1070 \mathrm{~cm}^{-1}$ peaks.

\subsubsection{Conclusions}

In conclusion, a straightforward hierarchical electrohydrodynamic pattern formation was used to produce well-defined sub-micron architectures in a tunable manner. Secondary and ternary EHD instabilities result in patterns widths that are considerably smaller (by about a factor of ten) than the primary structure size. The different generated structures display plasmonic properties which are controlled by the HEHD patterning parameters. This tunability allows the patterned substrates to be optimized for plasmon-dependent phenomena such as SERS. Indeed the different HEHD patterns show structure dependent SERS. For an optimized aspect ratio and geometry they give a 100-fold enhancement in SERS signals. Considering that each of the individual structures gives such high enhancements the HEHD patterned surfaces are a candidate platform for multiplexed SERS detection. In addition our results open up a range of further possible implementations in areas such as advanced photo-catalysis, photovoltaics, and bio-analytics. 


\section{Patterning of Crystalline Organic}

Materials via EHL

Structured crystalline polymer materials with anisotropic molecular orientation are of great interest in various applications, because of their special optical and electronic properties. Electrohydrodynamic lithography enables simultaneous structuring of organic crystalline materials while at the same time aligning the chains with respect to the formed structures. This gives rise to well-defined micro-patterns of crystalline polymers which are uniaxially aligned with the backbone parallel to the structure axis. Possible mechanisms inducing the vertical $c$-axis of the crystal lattice in EHD patterned films are discussed.

\subsection{Introduction}

Elegantly generated micro- or nano-structures are essential for the fabrication of highperformance devices which could be implemented in many technologically important fields ranging from integrated circuits through advanced optoelectronic displays to sensor arrays. The key to the realization of prospective applications relies on the availability of the optimal lithographic technique. Substantial progress has been made in developing micro-and-nano patterning processes $[23,76,130,178]$ during the past decade. While the vast majority of micro-to-nano fabrication schemes utilize resists of macromolecular nature, the extension towards additional material systems such as glassy, ceramic, ferroelectric and crystalline materials is of a high importance due to the functionalities such materials can offer. Particularly interesting is patterning of crystalline materials into desired structures due to their emergence as attractive candidates for photonic and electronic applications. [182] For instance, optically active, mostly semicrystalline conjugated polymers are competitive candidates for field effect transistors (FETs), solar cells and light emitting diodes. [46, 141] In particular, in thin film FETs, the mobility of charge carriers has been shown to be directly dependent on the orientation of semicrystalline chains. Morphological organization of crystalline polymer 
layers into regular surface structures has also been demonstrated to strongly influence selective adsorption behavior of proteins on surfaces. [48] The development of a direct route towards the generation of crystalline polymers patterns, and in particular, those of an anisotropic nature, still remains a considerable challenge. Device fabrication requires an establishment of versatile and straightforward lithographic method which both provides a simple way to create well-defined crystalline structures combined with promoting crystalline chain alignment in the generated patterns.

Initially established for highly viscous amorphous polymers, EHL [130] has been recently extended to a broad range of materials, including ceramics, [168] nanocomposites [43] and low-viscosity resists with fast pattern formation kinetics. [42] The generic nature of this low-cost technique makes it applicable to even larger class of materials. We demonstrate here the utilization of EHL method to generate well-defined patterns of crystallisable materials by means of electrostatic instabilities.

For a laterally varying $E_{\mathrm{f}}$, the EHL process yields high fidelity replication of variety of imposed structures by the master electrode. The small distance of downward protruding parts of the structured electrode locally enhances the electric field, leading to a faster destabilization and a lateral field gradient that focusses the instability towards the protrusions. Figure 7.1 illustrates the evolution of EHL line-pattern replication process of a topographically structured upper electrode. A thin confined film is liquefied and a constant voltage is applied to two electrodes (Fig. 7.1A). Initial instabilities are coupled to the lateral field variation and further focussed in the direction of highest electrostatic pressure and thereby guided towards the protruding structures of the top mask. This yields a pillar pattern spanning the capacitor gap at the locations of the smallest interelectrode distances (Fig. 7.1B), which eventually, by coalescence generates a replicated pattern of the upper electrode. The final structure is a positive replica of the master pattern (Fig. 7.1C) as opposed to the negative patterns achieved by conventional imprinting techniques. The final height of the polymer structures is determined by the plate spacing.

In this work we utilize the EHL technique to pattern crystalline polymers. This results in the formation of high-fidelity structures with an anisotropic orientation of polymer chains perpendicular to the substrate. Positional control of the generated morphologies can be adjusted by varying a number of experimental parameters, such as the initial film thickness, inter-electrode spacing, $E_{\mathrm{f}}$-strength, surface tension and lateral periodicity of the master electrode. The orientation of the crystalline $c$-axis is governed by key process parameters such as the electrostatic torque and the hydrodynamic flow. Because of the generic nature of the experimental approach, it is expected that a broad spectrum of crystalline materials can be patterned by using this technique. Along with the possibility of inducing anisotropy in crystalline materials structures, this consti- 


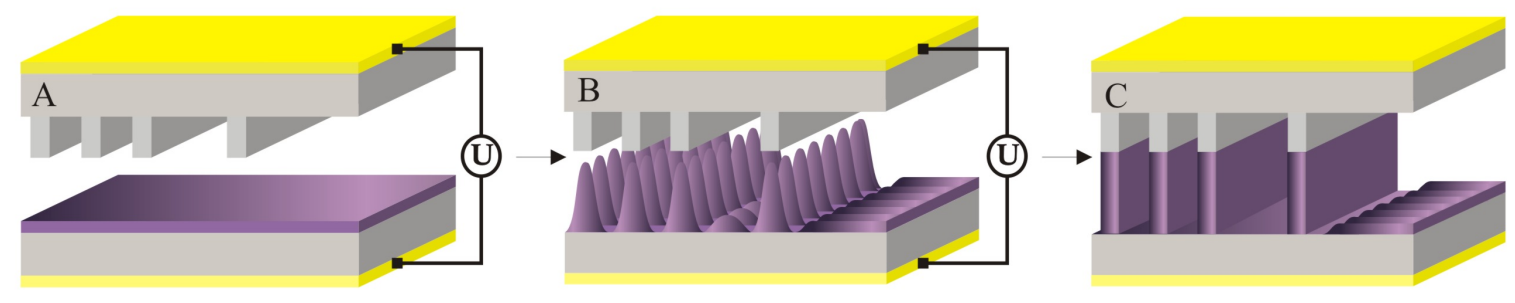

Figure 7.1: EHL pattern replication of a structured upper electrode: (A). A thin film in a capacitor assembly is liquefied and the amplification of instability is triggered by applying a voltage $U$. (B). The heterogeneous force field directs the electrohydrodynamic instability towards the protruding structures and generates liquid columnar-bridges between the two electrodes, and coarsening of the pillars results in a positive replica of the master pattern $(\mathrm{C})$. In unstructured regions, the film remains stable on a much longer timer scale.

tutes a promising route for the generation of functional sub-micrometer structures that were hitherto mainly accessible through rather cumbersome and expensive techniques.

\subsection{Experimental}

Polycaprolactone, (PCL) and Poly(Dimer Acid-co-1,6-hexanediol-co-Adipic Acid) (PDHA) were purchased from Sigma-Aldrich. Highly polished p-doped silicon ( $\mathrm{Si}$ ) wafers, with $<100>$ crystalorientation (Wafernet Gmbh, Eching, Germany) were used as substrates. Patterned silicon wafers were obtained from X-lith eXtreme Lithography, Ulm, Germany). Chloroform (Fischer Inc.) was used as a main solvent.

Film thicknesses ranging from $40 \mathrm{~nm}$ to $350 \mathrm{~nm}( \pm 10 \mathrm{~nm})$ were generated by spincoating onto a silicon wafer substrate from chloroform solutions with typical concentrations of $2-3 \%$ polymer by weight. Prior to spin-coating, the substrates were cleaned in a 'Piranha' solution consisting of $3: 1 \mathrm{H}_{2} \mathrm{SO}_{4}(98 \%): \mathrm{H}_{2} \mathrm{O}_{2}(30 \%)$, followed by thorough rinsing with deionised water and drying under nitrogen. Thereafter, substrates as well as electrodes covering the films were subjected to snow-jet cleaning immediately before film deposition and device assembly. Facing it, a wafer with planar as well as topographically structured surfaces, was mounted at specific distance using silicon oxide colloids as spacers, leaving a thin air gap, $d$ ( typically, $20-1200 \mathrm{~nm}$ between the mask and the film, Fig. 7.1). Both capacitor electrodes were electrically contacted using silver paint (Electrodag 1415M). The EHL experiment was based on mounting a structured electrode facing the liquefied film. A constant voltage of $80 \mathrm{~V}$ was applied across the two electrodes of the capacitor. In some of the EHL experiments, the upper electrode was substituted by a thick Kapton CR (DuPont) sheet with a thermally evaporated $50 \mathrm{~nm}$ thick Au layer on the reverse side. In order to avoid the air gap and establish conformal contact with the film, a thin layer of poly(dimethylsiloxane) 
(PDMS, Sylgard 184, Dow Corning) was spin-cast and thermally crosslinked on the front side of the Kapton sheet. Since, the presence of a thick Kapton layer and a dielectric significantly lowers the effective field across the polymer layer, a much higher voltage of $4.5 \mathrm{kV}$ was applied in such an assembly. After quenching to the crystallization temperature (i.e., temerature at which the solidification of crystalline structures occurs), the electric field was disconnected and the upper electrode was removed.

Light microscopy analysis was done employing Zeiss AXIO Imager. An Olympus BX51 optical polarizing microscope was used to investigate the optical textures of the samples. Optical anisotropy of oriented samples was evaluated with regard to variations of the intensity of reflected or transmitted light on a LM with a spectrometer attached (QE6500, Ocean Optics). The microscope halogen lamp acted as an illumination source for the spectroscopic measurements. Linear polarization measurements were obtained using achromatic polarisers (Thorlabs, Inc.) in cross and parallel configurations. The maximum intensity of reflected polarised light corresponds to the chains oriented parallel to the polarising filter $\left(45^{\circ}\right)$ and the minima when oriented at $\pm 45^{\circ}$ with respect to the filter $\left(0^{\circ}\right.$ or $\left.90^{\circ}\right)$. The sample topography was analysed by tapping mode atomic force microscopy (AFM) (Veeco, Dimension 3100). For transmission electron microscope (TEM) cross sectional imaging, the crystalline film has been embedded into a Spurr epoxy resin. The substrate was removed and the film was sectioned parallel to the formed structures (or substrate) using diamond knife in a Leica Ultracut Microtome, yielding sections with a thickness of $40-100 \mathrm{~nm}$. The cross-sections were analyzed using a FEI Technai 20 TEM $\left(L a B_{6}\right)$ at acceleration voltage of $200 \mathrm{kV}$ in diffraction mode. The obtained diffraction patterns were analysed using Digital Micrograph image (Gatan v3.3) processing software and JCEP/PCED simulations. [83]

\subsection{Results and Discussion}

\subsubsection{EHL of Crystalline Polymers}

The schematic representation in Fig. 7.1 summarizes the principle of the experimental procedure. To demonstrate the versatility of our approach, two crystalline materials systems were selected for the EHL patterning process: Polycaprolactone (PCL) and the amphiphilic Poly(Dimer Acid-co-1,6-hexanediol-co-Adipic Acid) (PDHA). The low transition temperatures and viscosities of the two samples (PCL: glass transition temperature, $T_{\mathrm{g}}=-59{ }^{\circ} \mathrm{C}$, crystallization temperature, $T_{\mathrm{c}}=47^{\circ} \mathrm{C}$, melting temperature, $T_{\mathrm{m}}=58^{\circ} \mathrm{C}$ and viscosity, $\eta=1.2 \mathrm{~Pa} \cdot \mathrm{s}$; PDHA: $T_{\mathrm{g}}=20^{\circ} \mathrm{C}, T_{\mathrm{c}}=35^{\circ} \mathrm{C}, T_{\mathrm{m}}=47^{\circ} \mathrm{C}$ and $\eta=0.55 \mathrm{~Pa} \cdot \mathrm{s}$ result in a brief annealing-quenching cycle, enabling high-speed EHL patterning. PCL is a biodegradable low-cost material which is often used for bio-sensing 
applications and bio-absorbable surgical devices. Generally, the liquefaction of a film is achieved via annealing the material above the softening point, followed by preserving the formed structures by cooling below $T_{\mathrm{g}}$ to the solid state. In the approach presented here the solidification is achieved by gradual quenching of the generated pattern to its crystalline state.

Films and experimental assemblies of both materials have been prepared in a similar manner. Initially, thin film was spin-cast onto a silicon wafer acting as a bottom electrode. The film was opposed by a second structured master electrode, leaving an air gap and forming a capacitor-like set-up. Subjecting the assembly to thermal annealing well above the melting temperature equilibrates the film, erasing all structural spherulitic features, thereby providing a homogeneous film prior to the EHL patterning. Then, a constant voltage is applied across the liquefied film. The electric field yields unstable undulations which are amplified, drawn towards the protruding structures, eventually bridging the two electrodes, resulting in a replication of the master electrode. Quenching the sample to below its melting point terminates the lithographic process while inducing the crystallization, generating well-defined crystalline structures. Figure 7.2 shows PCL square grid with a square width of ca. $2.9 \pm 0.4 \mu \mathrm{m}$ (Fig. 7.2A and B) and PDHA lines pattern (ca. $1.1 \pm 0.3 \mu \mathrm{m}$ in width) (Fig. 7.2E and F). Atomic force microscopy images reveal heights of $75 \pm 3.0 \mathrm{~nm}$ and $180 \pm 17 \mathrm{~nm}$ respectively. The master electrodes for replicated patterns have lateral feature dimensions of $3.0 \mu \mathrm{m}$ for cross-hatched structure (Fig. 7.2A, left) and $1.0 \mu \mathrm{m}$ (Fig. 7.2E, left) for the line pattern. The resulting patterns are formed over the entire electrode area and are highly reproducible. The electrostatically induced structure formation in the polymer film is not limited to wavelengths around the intrinsic maximally amplified wavelength, and a large range of lateral structures can be replicated for otherwise identical experimental parameters.

Well-ordered structures with nearly vertical walls, comprised of stacks of crystals are obtained for both PCL (Fig. 7.2B) and PDHA (Fig. 7.2F). Notice, while the PCL crystalline texture of the formed patterns can be straightforwardly resolved by optical microscopy images, polarized optical microscopy (POM) is required to reveal the crystalline nature of the PDHA lines pattern (Fig. 7.2F, inset). This suggests that the grain size of crystals forming the PDHA pattern is finer than of PCL.

\subsubsection{EHD-Induced Crystallization}

To gain additional insight regarding the crystalline orientation in the formed structures, electron diffraction patterns of thin sections was performed. The electron beam was normal to the film surface. In this transmission geometry only reflections from lattice planes with their normal vector in the film plane are observable. The diffraction 

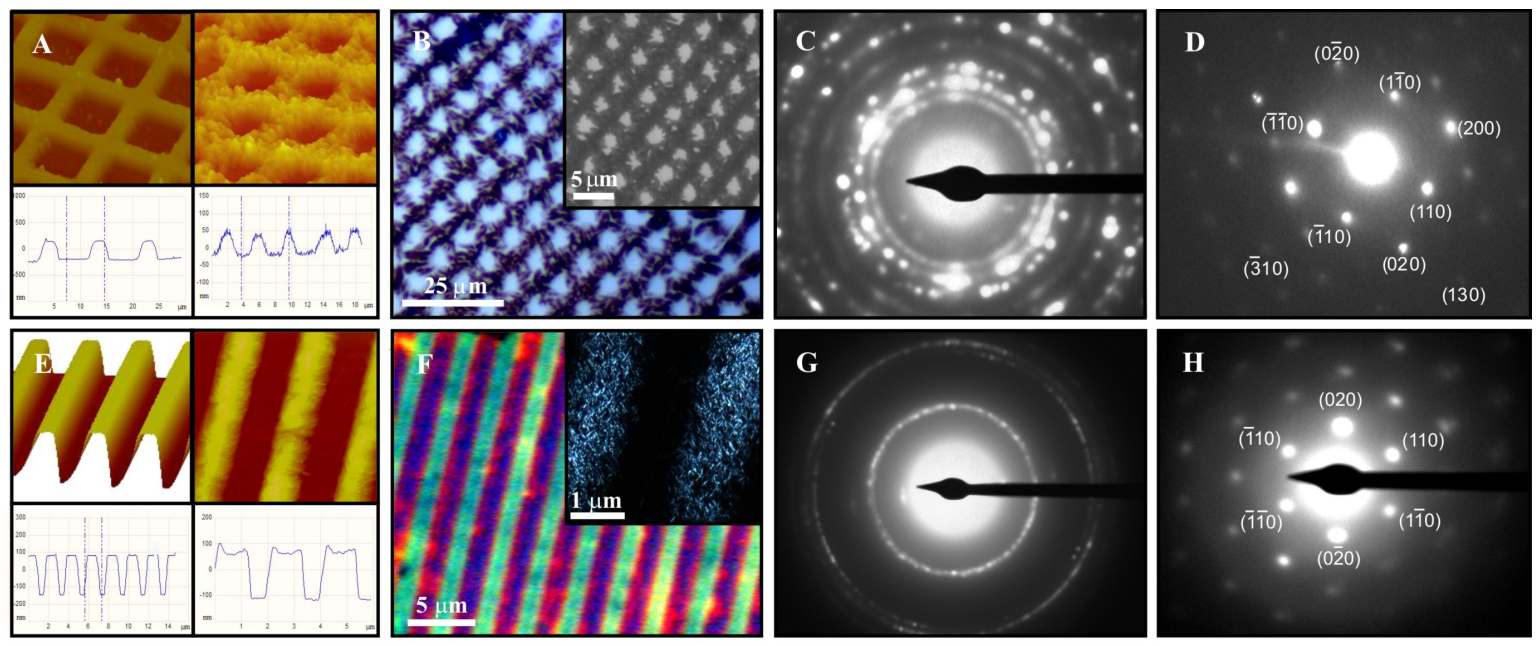

Figure 7.2: AFM images of a master electrode (left) and the replicated structures (right) of PCL (A) and PDHA (E) with corresponding cross-sections determining lateral and vertical dimensions of the pattern. Optical micrographs reveal well-defined crystalline EHL-generated patterns of PCL (B) and of PDHA in (F). The POM images enable the visualization of the crystalline texture of the line pattern (F, inset). Diffraction patterns for a large area of the PCL (C) and PDHA (G) patterns. Selected area diffraction pattern obtained with an aperture of $3.0 \mu \mathrm{m}$ of a single PCL crystal in (D) and with a $1.0 \mu \mathrm{m}$ aperture diameter of a single PDHA crystal in $(\mathrm{H})$.

patterns in Fig. 7.2C and $\mathrm{G}$ were obtained from patterned PCL- and PDHA-films, respectively. The illuminated area was $350 \mu \mathrm{m}^{2}$, i.e. for both the cross-hatched and the line structures approximately $5-7$ lines were imaged (cf. Fig. 7.2E and F). For both replicated structures of PCL (Fig. 7.2C) and PDHA (Fig. 7.2G) a set of nested rings pattern is formed in the diffraction plane indicating a polycrystalline nature. However, all observable diffraction rings for PCL can be indexed as (hk0)-reflections of the orthorhombic PCL-crystal structure. [14] The first two (most internal) reflections can be indexed as (110) and (200) reflections, followed by (210) and (020) planes, the (120) and (310) planes for the fifth ring and the (400) and (410) planes for the sixth and seventh rings, respectively. We have recorded several diffraction patterns from different areas of the patterned PCL films and all of them showed (hk0)-reflections only. It can be also observed that while the diffraction ring of the PCL pattern is made up of discrete spots, PDHA diffraction produces a more continuous ring pattern, indicating smaller crystal grains in comparison to PCL. When imaging a smaller area, individual singlecrystalline grains become apparent. As selected area diffraction (SAD) pattern in Fig. $7.2 \mathrm{D}$ was recorded from $3.0 \mu \mathrm{m}$-wide area on the PCL sample. The different spots can be assigned to different (hk0)-reflections. The absence of (h00) and (0k0)-reflections with odd $h$ and $k$ as well as the lattice parameters $a=0.74 \mathrm{~nm}$ and $b=0.50 \mathrm{~nm}$ were in agreement with the literature. [14] Thus, electron diffraction results demonstrates that PCL-crystals were uniaxially aligned during the patterning process with the $c$-axis, i.e. 
the chain axis, parallel to the film normal, with a grain size of approximately $3.0 \mu \mathrm{m}$. In a similar manner, a SAD pattern was obtained from a single PDHA crystal (Fig. $7.2 \mathrm{G}$ ) with a selected diffraction area of $1.0 \mu \mathrm{m}$. Both SAD patterns correspond to single crystal structures although of different dimensions, which is in agreement with the previous observation of Fig. 7.2D,G. In Fig. 7.2H, the spots form a slightly distorted hexagon for PDHA spots, which were assigned to the (020) and (110) crystal planes. All electron diffraction patterns were acquired in the normal projection relative to the film normal. This analysis indicates that both the EHL patterned materials' crystals exhibit a $c$-axis almost parallel to the incident electron beam, showing chains that were highly aligned parallel to the $E_{\mathrm{f}}$ which is also shear flow direction during EHL pattern formation.

\subsubsection{Possible Mechanism for the Crystal Orientation}

To address the origin of the crystal alignment, three possible alignment mechanisms leading to the observed $c$-axis orientation have to be considered. For EHD lithography structure formation of nanocomposites of carbon nanotubes dispersed in a polymer matrix we have shown that a combination of shear flow and an electric field induced torque caused by the anisotropic shape of the nanotubes lead to an alignment of the nanotubes. [43] Because of the absence of structure in the liquid state during pattern formation, the latter effect which is also well known to align cylindrical or lamellar structures in block copolymers [167] can be excluded for the present case. In difference to blockcopolymers and nanotube composites, the present system is homogeneous during EHD pattern formation, and crystallization sets in only after termination of the electric fielddriven flow. We anticipate three possible mechanisms for the interplay of the electric field with the crystallisation process. The first possible mechanism is the extension and the alignment of the chains by the elongational component of the flow during pattern formation. This prealignment facilitates the formation of $c$-axis oriented crystals upon quenching below the melting point. The second possible mechanism relies on the anisotropic dielectric properties of crystals and the resulting orientation dependent free energy of a crystal in an electric field, [134] similar to what has been studied for the case of polymers with anisotropic magnetic properties in magnetic fields. [31, 71] This causes the crystal nuclei to rotate into a preferred orientation upon nucleation. Third and last, it has been demonstrated that polymers crystallizing in confined geometries, with no external field or flow acting can show macroscopic alignment due to a kinetic "gate effect". [148] In this case, the two dimensional confinement suppresses branching as well as spherulite formation and selects specific crystal orientations during growth. Thus, in the kinetic selection mechanism, the growth rates in the different growth directions lead to an overall dominant crystal orientation, resulting in uniform uniaxial 
crystal orientation. Similarly, in our film geometry if the (hk0)-lattice plane normals are in the plane of the film (i.e., with the backbone of the chains aligned approximately perpendicular to the film surface), polymer crystals grow over larger distances in the plane of the film while those growing in the perpendicular direction will impinge on the surface.

Before testing the three mechanisms experimentally, the first two mechanisms, i.e. the chain alignment by elongational flow and alignment induced by the anisotropic dielectric constant are discussed below. Flow induced crystallization resulting in higher crystallization rates and aligned crystals, has been studied extensively in the past. [118] It has been established that for a given molecular weight of the polymer, a critical strain rate exists above which flow induced effects show up [118, 143] and that this critical strain rate decreases with increasing molecular weight. In most cases shear has been applied at the crystallization temperature. For PCL with a molecular weight of $M_{\mathrm{n}}=10,000 \mathrm{~g} \mathrm{~mol}^{-1}$ it has been demonstrated that applying shear stress during a quench from $T=80^{\circ} \mathrm{C}$ to $T=55^{\circ} \mathrm{C}$ can significantly reduce the crystallization time. [38] Furthermore, for PCL with $M_{\mathrm{n}}=80,000 \mathrm{~g} \mathrm{~mol}^{-1}$ it was found that shear rates as low as $\dot{\gamma} \leq 1 \mathrm{~s}^{-1}$ applied during a quench from $T=80^{\circ} \mathrm{C}$ to $T=46^{\circ} \mathrm{C}$ enhance crystallization. In difference to these examples, elongational flow was active only during pattern formation at a temperature of $T=75^{\circ} \mathrm{C}$ in our experiments.

Figure 7.3 shows the elongational flow for the intermediate stages of the pattern formation of a single column. The image shows an emerging pillar surrounded by a depletion zone and a rim. The material from the depletion zone is drawn towards the column while the surrounding film remains intact. This polymer flow results in a decrease in the thickness of the region surrounding the forming column. During the formation of the elevated rim and the expansion of the central column, the depletion zone is drained and exposes the substrate surface. Once the substrate is exposed, the polymer retracts from the surface and accumulates in the rim and in the column. Above the glass transition temperature, polymer fluids used in our experiments can be described as incompressible viscous liquids and in the zero frequency limit they are Newtonian i.e., the shear force per unit area is proportional to the local velocity gradient. The corresponding flow gives rise to a Poiseuille-type velocity profile in the liquid layer (Fig. 7.3). [49, 127] In the lubrication approximation, [101] assuming zero slip at the substrate (resulting in a zero velocity) and no lateral stresses at the film surface: higher shear stresses evolve in the thinning regions of the film during the intermediate stages of the pillar formation, Therefore, the shear gradient is maximal at the depletion zone between the emerging pillar and the surrounding rim, possibly giving rise to flow alignment of the stretched polymer chains.

The timescale on which a chain remains in its stretched state may be roughly 


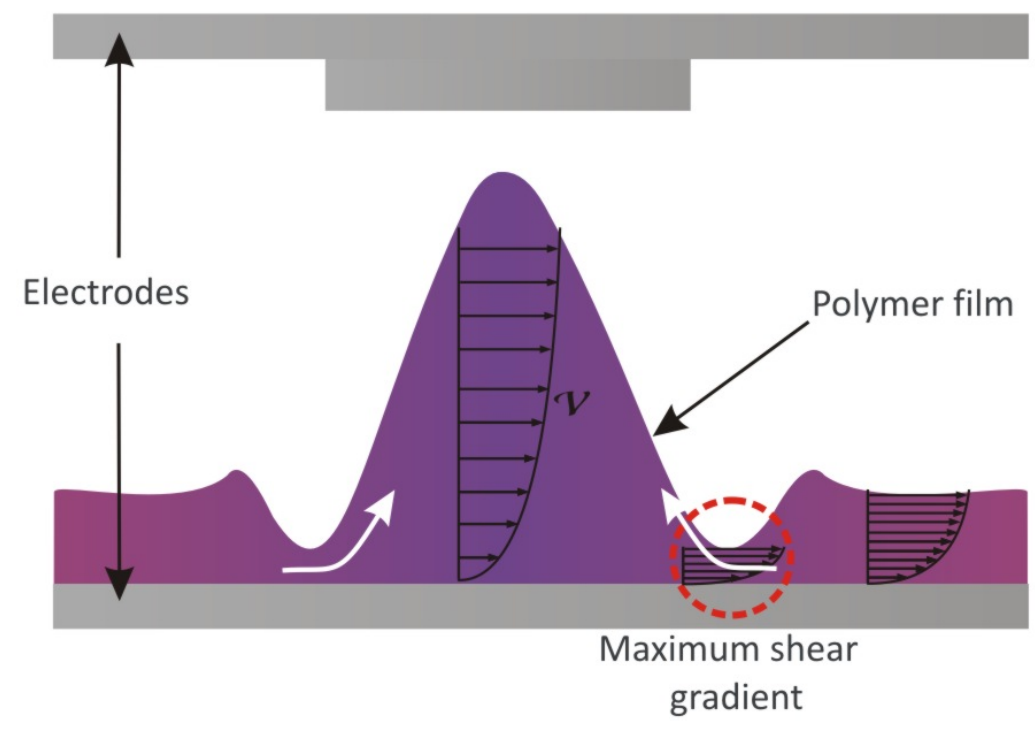

Figure 7.3: Schematic of the emergence of a column surrounded by rim during intermediate stages of the EHL pattern formation. The polymer material flows towards the emerging column from a direction perpendicular to cross-section. The dotted circle indicates the area of a maximum shear rate. The Poiseuille-type flow is indicated by a parabolic flow profile with the velocity profile indicated by the arrows. The cone shape of the intermediate stage is the result of the amplification of the wave maximum incorporating the surrounding polymer material.

estimated by assessing the reptation time:

$$
\tau_{\text {rep }} \approx \frac{<L>^{2}}{D}=\frac{\zeta b^{2} N^{3}}{k_{\mathrm{B}} T N \mathrm{e}}
$$

with the friction coefficient $\zeta=6 \pi \eta R_{\mathrm{g}}$ and the polymer radius of gyration $R_{\mathrm{g}}=a \sqrt{N}$. For the PCL polymer the degree of polymerization was $N \approx 88$. Using $a=l_{\text {monomer }} \approx$ $1 \mathrm{~nm}$ and $\eta=1.2 \mathrm{~Pa} \cdot \mathrm{s}$ we find at $T=75^{\circ} \mathrm{C}$ a value of $\tau_{\text {rep }}$ of around ten milliseconds.

We further estimate the second mechanism relying on the anisotropic dielectric constant in the crystalline state. The following calculation is very similar to the calculation in Ref. [71], where a polymer-crystal with anisotropic magnetic susceptibility in a magnetic field is considered. The free energy of a crystal with volume $V$ in an electric field $\vec{E}$ is given by

$$
F=F_{0}-2 \varepsilon_{0} \int_{\mathrm{V}} \vec{E} \tilde{\varepsilon} \vec{E} d^{3} r
$$

with the matrix $\tilde{\varepsilon}$ which describes the anisotropic dielectric properties. If we define $\vartheta$ as the angle between the electric field direction and the $c$-axis (i.e. the chain axis for polymer crystals) and further assume $\varepsilon_{a} \approx \varepsilon_{b}<\varepsilon_{c}$ the angle-dependent part of the free energy can be calculated to give $F(\vartheta)=-V / 2 \varepsilon_{0} E^{2} \triangle \varepsilon \cos ^{2} \vartheta$, with $\triangle \varepsilon=$ 
$\varepsilon_{c}-0.5 \cdot\left(\varepsilon_{a}+\varepsilon_{b}\right)$. Thus, an orientation of the $c$-axis of crystal along the electric field direction is preferred. Comparing the difference $\triangle F=F\left(\vartheta=90^{\circ}\right)-F\left(\vartheta=0^{\circ}\right) \sim k_{B} T$ with the thermal energy gives an estimate for the minimum volume of a crystal that aligns in the electric field. Furthermore, the time needed to rotate a sphere with dielectric anisotropy $\triangle \varepsilon$ in a viscous liquid with viscosity $\eta$ in an electric field with strength $E$ is given by [71]

$$
\tau=\frac{6 \eta}{\varepsilon_{0} \triangle \varepsilon E^{2}}
$$

In the absence of values for $\varepsilon_{i}(i=\mathrm{a}, \mathrm{b}$ c) for PCL, we have to resort to several approximations to estimate the minimum volume and the rotation time. The crystal structure of PCL in the a-b plane is very similar to polyethylene (PE) and PCL, like $\mathrm{PE}$, forms negatively birefringent spherulites, [126] i.e. the refractive index along the chain axis is highest. If we approximate $\varepsilon_{i}(P C L) \approx \varepsilon_{i}(P E) \approx n_{i}^{2}(P E)$ we can estimate the above quantities. The refractive indices of $\mathrm{PE}$ are $n_{a} \approx n_{b} \approx 1.52$ and $n_{c} \approx 1.55$. For $E=80 \mathrm{~V} / 75 \mathrm{~nm}$ we find at $T=60^{\circ} \mathrm{C}$ a volume of $V \approx(1 \mathrm{~nm})^{3}$ at which the difference in free energy $\triangle F=F\left(\vartheta=90^{\circ}\right)-F\left(\vartheta=0^{\circ}\right)$ becomes comparable to the thermal energy. With the viscosity $\eta=1.2 \mathrm{~Pa} \cdot \mathrm{s}$ of PCL at $T=70^{\circ} \mathrm{C}$, a rotation time of $\tau=10^{-4} \mathrm{~s}$ was obtained. Both, the small volume and the fast rotation indicate that this alignment mechanism may well be active in our experiments.

To experimentally address, which of the three proposed alignment mechanisms is dominant, an additional series of measurements was performed. Since, PCL is a well-studied crystalline structure we will limit the further discussion of the plausible alignment mechanisms to this polymer only. First, to gain insight into kinetics of chain alignment, two new sample types were prepared. First, a thin PCL film was annealed above it $T_{\mathrm{m}}$ and quenched back to its crystalline state and further analyzed by diffraction TEM. The corresponding pattern is shown in Fig. 7.4B. Second, instead of leaving an air gap in the capacitor, the top electrode was brought into direct contact with the crystal polymer layer (see experimental section for full details). In this device geometry, hydrodynamic flow is suppressed, isolating the rotation of anisotropic crystallites in the electric field as possible mechanism for $c$-axis alignment. The corresponding TEM diffraction pattern is shown in Fig. 7.4C.

Thin polycrystalline films with randomly oriented crystals exhibit a characteristic electron diffraction pattern (DP) consisting of a sequence of rings corresponding to the structure factor. The absence of rings, on the other hand, indicates the presence of a crystal alignment parallel to the electron beam. The intensities of the electron diffraction rings from thin polycrystalline films depend on the orientation distribution of the crystallites contributing to the DP. The extent of preferred orientation can be determined by measuring the diffraction ring relative intensities even when the preferred 
orientation is only partial. Aiming to extract the preferred orientation from electron diffraction ring patterns of polycrystalline specimens, the first step in analysis requires the generation of intensity ratios for a sample with random crystal orientation. The experimental ring intensities showing greatest deviation from the calculated values for random orientation should indicate which zone axes are in greater or smaller abundance compared to a sample in which the crystallites are randomly oriented. [171] Here, relative X-ray intensities of a powder pattern of randomly oriented PCL [14] were used as a reference. The relationship between the X-ray and electron diffraction intensities allows for such a comparison. [122] The profile of the integrated intensity distribution was calculated for each experimental sample (i.e., EHL patterned PCL (S1), PCL film subjected to an electric field only in the absence of an air gap (S2) and the film crystallized without an electric field (S3), based on the method of extraction of peak intensity similar to the powder X-ray diffraction method and calculation of average intensities for each ring as described in [83, 84].

Examination of the DPs shown in Fig. 7.4 reveals that the relative intensities of the various rings in S1 (Fig. 7.4A), S3 (Fig. 7.4B) and S2 (Fig. 7.4C) vary markedly from those observed in a sample with crystal random orientation, [14] (Fig. 7.4D). The only rings seen in Fig. 7.4A are from (hk0) reflection planes and are stronger compared to the sample with randomly oriented crystals. These intensities stem from a crystal orientation perpendicular to the plane of the film in EHL patterned PCL samples.

Noted that the measured intensities of certain rings in Figs. 7.4B and $\mathrm{C}$ differ from the relative intensities in random crystal alignment (Fig. 7.4D). In particular, the (020), (120), (400), (410) and (202) rings of S3 and (011), (021), (027), (310) and (127) rings of S2 are enhanced. The majority of the rings with a noticeable increased intensity correspond to families of Bragg planes of the type (hk0) in the case of S3 (i.e., supporting the kinetic effect), and (0kl) in the case of S2 (i.e., supporting the electric field effect). While (hk0) planes are parallel to the electron beam and the sample normal, the (0kl) planes are inclined with respect to this axis (Fig. 7.4D-E). Several rings are missing in both S2 and S3 and for most of the observable reflections the relative intensities are larger compared to random crystal alignment (Fig. 7.4D). Comparing these samples with the diffraction pattern analysis of the EHL patterned structures reveals a lower degree of orientation. Texture can be observed in both S2 (Fig. 7.4B) and $\mathrm{S} 3$ (Fig. 7.4C) samples.

For an electron beam striking the plane of the film at perpendicular incidence, the surface normal is parallel to the beam. Since the [001] axis is perpendicular to the sample surface, families of Bragg planes are inclined with specific angles with respect to this axis. For the cubic system the angle $\alpha$ at which a plane is inclined to this axis 
is given by [160]:

$$
\tan \alpha=l / \sqrt{h^{2}+k^{2}}
$$

Partially oriented films (Figs. 7.4B and C) have zone axes inclined to the electron beam axis, but with insufficient orientation to cause the disappearance of rings. While planes having $\alpha$ in the range $0^{\circ}$ to $45^{\circ}$ (Fig. 7.4E) reflect with relatively high intensities,
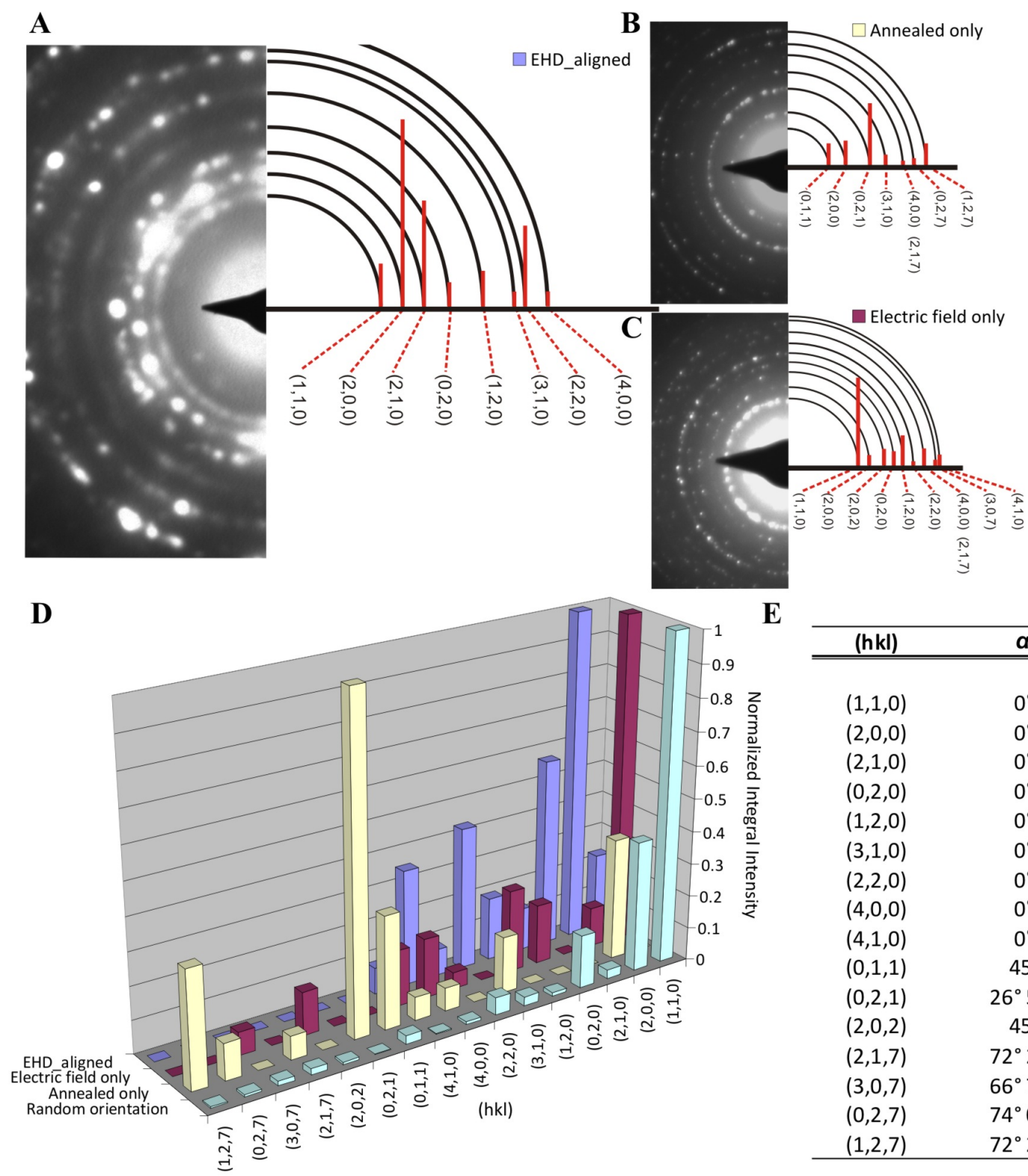

$\mathbf{E}$

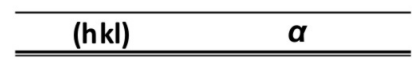

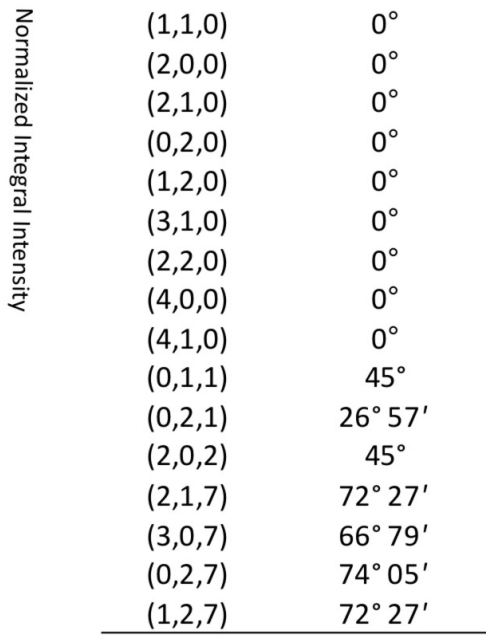

Figure 7.4: Electron diffraction patterns and corresponding relative integral intensities of: (A) vertically ordered crystalline morphology within EHL generated structure, (B) a thin film crystallized without an applied electric field and (C) a sample with an applied electric field, but without an airgap. Comparison of relative intensities of diffraction patterns in (A)- $(\mathrm{C})$ to randomly oriented PCL crystallites [14]. (E). Orientation distribution of reflecting planes and inclination angles with respect to the [001] orientation. 
the reflection from the $(\mathrm{hk}, 7)$ planes for which $\alpha>50^{\circ}$, have much decreased intensities.

The results from Fig. 7.4 allow the following conclusions: While patterns of Fig. $7.4 \mathrm{~A}$ are indicative of a crystal direction perpendicular to the plane of the film, the diffraction patterns of Figs. $7.4 \mathrm{~B}$ and $\mathrm{C}$ also indicate a non-random texture of the crystals in the films, with an orientation distribution ranging from $0^{\circ}$ to $\simeq 74^{\circ}$. The sample crystallized in presence of an electric field only (Fig. 7.4C) consists dominantly of crystals with a $<\mathrm{hk} 0>$ direction aligned with the surface normal. $<0 \mathrm{kl}>$ crystal orientations with nonzero 1 index, however, prevail in the case of the sample analyzed in Fig. 7.4B. Moreover, since the intensity of the hkl ring is proportional to the multiplicity of the occurrence of the hkl planes, a higher relative intensity of the (hk0) reflections Fig. $7.4 \mathrm{C}$ in comparison to Fig. 7.4B point towards a higher degree of vertical orientation than in the case of the film crystallized with no electric field, but lower relative to the EHL patterned structures.

The above discussion based on the experimental observations indicates that there are several effects driving crystal alignment. EHL pattern formation gives rise to a lateral shear flow into the evolving structures during which the chains can be stretched and aligned in the flow direction. However, following the completion of the patterning process the sample was typically cooled for several minutes to a temperature at which the crystallization occurred. In the absence of additional forces, it seems unlikely that the chains remain in their stretched state until the onset of the crystallization. Forces arising only from hydrodynamic convection and elongational flow are therefore unlikely to cause crystal alignment. Electric fields were shown to align block copolymer nanodomains parallel to the electric field lines for fields exceeding $30 \mathrm{kV} / \mathrm{cm}$. [97] In our setup, a voltage on the order of 10 volts was applied between the electrodes. Together with the small interelcrode spacing, this results in very high electric fields on the order of $10^{8} \mathrm{~V} / \mathrm{m}$. The domination of (hk0) over the (hkl) reflections in the presence of an electric field in the absence of flow indicates that although there are areas with different orientations, there is an overall orientation of the crystal $c$-axis normal to the substrate. However, comparing the film subjected to only an electric field to the EHL patterned sample indicates a smaller extent of orientation to the surface normal in the case of a mere electric field presence. These results imply that electric field does play a significant role in aligning the c-axis of crystals, but alone it is insufficient to cause an overall reorientation. The coupling of the applied electric field to an additional effect should be therefore, considered. The diffraction patterns analyzing the kinetic mechanism reveal mostly partial orientation with larger inclination from the orientation direction. However, it seems that the (to some extent weaker) kinetic effect also contributes to the selection of the fastest growing mode along the preferred (perpendicular) direction, 
thus inducing an additional factor in favour of vertical orientation (S2). The dominant relative intensity of the $(0 \mathrm{kl})$ direction suggests on the other hand, a rather small extent of a vertical orientation in the case the kinetic effect. Therefore, in our case it seems that coupling of the electric field and the confining kinetic effect is insufficient to yield a high degree of vertical alignment as observed for EHL patterned samples. Moreover, the alignment of liquid crystals in the presence of an electric field in a confined geometry has been studied, suggesting that the desired initial alignment of the liquid crystal in the absence of an external field, such as an electric field is generally achieved by appropriate surface treatment of the confining substrates, such as by applying a surface-director on the confining substrate surfaces. The initial liquid alignment is therefore defined by solid surface/liquid crystal interactions. The orientation of the liquid crystals adjacent to the confining surface is transferred to the liquid crystal molecules, thus imposing essentially the same alignment to all liquid crystal bulk molecules. In this context, it seems reasonable to suggest that the crystalline films confined between two electrodes are initially subjected to the kinetic gate effect resulting in a small extent of vertical orientation . Following the kinetic gate effect, the shear flow in a confined geometry perturbs the formation of randomly oriented crystalline chains while stretching and pre-aligning the chains, thus acting as the director field predefining the alignment direction. Further, the dipole moment of the anisotropic elongated crystalline chains couples to the strong electric field in the capacitor which upholds the structures in the final aligned form. The resulting morphology is therefore, a result of coupling of all the above discussed effects aligning vertically in the structures caused partially by the preliminary kinetic effect and the flow during the structures formation and partially, by the action of the electric field.

The creation of well-defined submicron patterns comprised of aligned crystalline chains perpendicular to the substrate described here provides a proof-of-concept showing that the EHL lithographic approach constitutes a cost-effective and straightforward approach to directly pattern and align semicrystalline polymers. One can readily envision the useful application of the present patterning method. For instance, orienting functional, conjugated polymers, such as functionalized polythiophene (P3HT), using electrohydrodynamic approach may have implications for field-effect transistors, light emitting diodes and sensors. Furthermore, this route might enable applications in liquid crystal display technology, such as multidomain vertically aligned display modes leading to improved viewing angles in displays. [89] Morphological control over oriented semiconductor architectures that can be achieved with the presented approach yields an interfacial area between electronic components and continuous pathways for charge transport. The EHL method is therefore, a competitive candidate for materials-based applications and may be of interest in the development of high performance devices. 


\subsection{Conclusions}

This study introduces a straightforward technique to pattern semi-crystalline organic materials and at the same time induce well-defined crystalline order. The exposure of liquefied films to a heterogeneous electric field gives rise to submicron sized patterns which can be lithographically controlled. Therefore, EHL allows tuning and manipulation of the position of the formed patterns as well as the vertical alignment of the $c$-axis of the crystalline polymers. This patterning technique presents a successful combination of bottom-up and top-down approaches based on the electrohydrodynamic generation of surface patterns in thin crystalline films with the uniaxially aligned crystals with the backbone parallel to the surface normal. Within each configuration the crystalline domains are oriented parallel to the surface normal, which appears to be induced by the combined effects of initial kinetic selection of the perpendicular growth direction in the confined geometry, the shear (pre)alignment of crystals during EHL pattern fabrication and its coupling to the strong electric field generated in the capacitor.

The application of EHL patterning to crystalline materials and in particular, inducing an anisotropic structure of polymer crystals is attractive because it extends this technique towards functionalities that can not be achieved by using amorphous or polycrystalline materials. The orientation of crystals within the structures will strongly influence their mechanical, electronic, optical and ferroeelctric properties. Moreover, the possibility of obtaining aligned functional crystalline materials that are patterned with sub-micrometer resolution introduces a spectrum of interesting prospects. Singlecrystal semiconductor architectures, for instance are important in a range of applications requiring a large surface area, morphological control and higher charge carrier mobility. This approach may well be a herald of a general strategy for the design and fabrication of functional semiconducting materials with desired nanopatterns, orientations, and shapes which can be employed in the development of advanced miniaturized devices with potential applications in photocatalysis, biochemical sensors, photochromism and display technology. 


\section{Electrohydrodynamic Lithography of a}

\section{Conducting Polymer}

An increasing number of technologies require the fabrication of conductive micro and nanostructures over large areas. Here we introduce a simple electrohydrodynamic lithographic technique for patterning a conductive polymer directly on a substrate with high fidelity. Although theoretical studies addressing the pattern generation have predicted EHD pattern formation in conducting liquids, [106, 107, 109] there are no experimental studies of EHD patterning of conductive films. We illustrate the generality of this robust, low-cost method by patterning thin polypyrrole (PPy) films via electricfield induced instabilities, yielding well-defined conductive structures with feature sizes ranging from tens of micrometers to hundreds nanometres. Exploitation of conductive polymer induces free charge suppression of the field in the polymer film, paving the way for accessing scale sizes in the deep submicron range. Controlled pattering of conductive polymer structures at micro and nanoscale demonstrated in this study might herald a route towards various submicron device applications in supercapacitors, photovoltaics, sensors and electronic displays.

\subsection{Introduction}

Conductive polymers (CPs) combine properties of polymers with the electrical and optical properties of metals. Straightforward processing, tuneable optoelectronic properties via molecular design and the possibility to modify conductivity by doping, are only few of the advantages attributed to the CPs. Considerable research has been directed towards studying CPs aiming for their potential implementation into a variety of functional devices, including field effect transistors (FETs), [66, 170] organic light emitting diodes (OLEDs), integrated circuits, chemical and biological sensors [65] and electrochromic devices. [146]

In order to take full advantage of the functions of $\pi$-conjugated macromolecules and in particular, for generation of flexible organic electronic devices, patterning of 
CPs into structures ranging from $100 \mu \mathrm{m}$ to sub-100 nm is required. Moreover, a good adhesion of the CPs films and structures on the substrate is essential to realize the full potential of CPs patterns for their future integration into applied devices. [28] Conducting polymer micro and nanostructures can, for instance, be used for red, green or blue (RGB) pixels in multicolour OLED displays or interconnects in all-polymer integrated circuits. [29]

Routes for patterning CPs have been explored extensively, and the most prominent techniques are soft-lithography, [103] electron beam lithography, [115] photochemical patterning by photolithography [88, 132] and nanoimprint lithography. [46] Hitherto, however, most of the existing approaches to pattern CPs have limitations in certain aspects regarding resolution, position control, versatility and reproducibility. While some conventional lithographic techniques approach their physical limits, others are incompatible with CPs due to the poor adhesion between the polymers and the substrate. In light of the complexity involved in obtaining high resolution registration from soft-lithography, together with an increasing need to develop low-cost and high throughput methods for patterning conductive polymer films, interest in alternative patterning processes has grown. Generation of high-fidelity submicrometer architectures of CPs in a controlled manner would enable large scale fabrication of advanced devices.

In the present work, we introduce elegant, straightforward EHL method for patterning conductive polymers. This process provides a single-step and cost-effective approach for direct patterning of conjugated polymers on solid substrates, generating a variety of feature sizes ranging from tens of micrometres to hundreds of nanometres. [42, 127, 130, 131] The EHL concept exploits an instability induced by an applied electric field across the liquefied polymer-air bilayer sandwiched between two-electrodes in a capacitor-like device. In the case of dielectric polymers, the electric field causes the energetically unfavorable build-up of displacement charges at the dielectric interface, leading to the alignment of the dielectric interface parallel to the electric field lines which lower the electrostatic energy. In a homogeneous electric field, this typically results in pillar structures which span the two electrodes. Since, the total potential difference generated by the dipole layers at the interface is suppressed across the liquid layer, the driving force of the pattern formation in the case of a leaky dielectric polymer subjected to the EHL patterning lies in the electric field in the air gap, $E^{1}$. [107] A sub-ambient pressure within the film balances the electrostatic force due to the field in the air gap on the polymer-air interface, placing the film in tension, and therefore, generating the origin of the EHL instability. The intrinsic length scale of instabilities in thin films is on the order of microns. In order to exploit this natural structure formation process, it is important to, first, control the resulting pattern and, second, decrease 
the length scale to technologically interesting feature sizes. Both requirements are fulfilled by imposing a laterally heterogeneous electric field with variations smaller than the intrinsic wavelength. Such a lateral field variation is typically achieved by using a topographically structured mask as one of the electrodes, thus enabling the generation of a broad variety of structures on different length scales. EHL can be used to fabricate patterns in a wide variety of polymers and composites using both featureless and topographically structured masks. Positional and dimensional control of the generated morphologies can be tuned by varying a number of experimental parameters, such as the initial film thickness, inter-electrode spacing, applied voltage, surface tension and lateral periodicity of the master electrode. This facile EHL technique opens up a new avenue for patterning CPs for various applications including for example, transistors, coatings, solar cells and in microelectronics.

\subsection{Experimental}

All the chemicals were purchased from Sigma-Aldrich and used without further purification. PPy soluble in organic solvents has been synthesized (with slight modifications) following the procedure found in [34]: 0.149 of dried pyrrole monomer was added to a solution comprised of $0.0745 \mathrm{~mol}$ of dodecylbenzene sulfonic acid (DBSA) dissolved in $300 \mathrm{ml}$ deionized water (DW) and dynamically stirred for 30 minutes. $0.35 \mathrm{M}$ of aqueous ammonium persulfate solution was added to this mixture and left to react for 18 hours under continuous stirring. Addition of methanol stopped the reaction and the final solution was filtered to obtain black PPy powder. The PPy was washed several times with methanol and excess of DW followed by filtering, eventually yielding pure PPY. The molecular weight of PPy was $157 \mathrm{~kg} / \mathrm{mol}$ and glass transition temperature was $98^{\circ} \mathrm{C}$.

Highly polished $p$-doped silicon ( $\mathrm{Si}$ ) wafers, with $<100>$ crystal orientation (Wafernet Gmbh, Eching, Germany) covered by $100 \mathrm{~nm}$ or $300 \mathrm{~nm}$ thick oxide layer were used as substrates. Patterned silicon wafers were obtained from X-lith eXtreme Lithography, Ulm, Germany). Chloroform (Fischer Inc.) was used as a main solvent.

Film thicknesses ranging from $80 \mathrm{~nm}$ to $200 \mathrm{~nm}$ were generated by spin-coating onto a silicon wafer substrate (dimensions of $1 \times 1 \mathrm{~cm}^{2}$ ) from chloroform solution with typical concentrations of $2-3 \%$ polymer by weight. Prior to spin-coating, the substrates were cleaned in a 'Piranha' solution consisting of 3:1 $\mathrm{H}_{2} \mathrm{SO}_{4}(98 \%): \mathrm{H}_{2} \mathrm{O}_{2}$ (30\%), followed by thorough rinsing with deionised water and drying under nitrogen. To ensure the integrity of the formed structures, patterned electrodes were rendered hydrophobic by the deposition of a 1,1,1,2H-perfluorodecyltriclorosilane self-assembled monolayer to reduce the adhesion between the mask and the polymer. Thereafter, substrates as well 
as electrodes covering the films were subjected to snow-jet cleaning immediately before film deposition and device assembly. Facing it, a topographically structured electrode, was mounted at specific distance using silicon oxide colloids as spacers, leaving a thin air gap, $d$ (between the mask and the film, Fig. 8.1A). Capacitor electrodes were electrically contacted using silver paint (Electrodag 1415M). A constant voltage of $40-$ $80 \mathrm{~V}$ was applied across the two electrodes in the capacitor, and the temperature of the device was raised above the $T_{\mathrm{g}}$ of the polymer film. After quenching the samples to room temperature, the electric field was disconnected and the upper electrode was removed. The sample topography was analyzed by OM and AFM. All the measurements were performed in air under ambient conditions using the tapping mode

\subsection{Results and Discussion}

Owing to its high electroconductivity, good stability in ambient conditions and facile processability, the intrinsically conducting PPy polymer has been a topic of an extensive research concurrently focussing on practical applications and on synthesis. PPy can be synthesized by electrochemical or chemical polymerization methods. One of the main limiting factors has, however, been its non-solubility in any solvent due to the strong intermolecular interactions of the heterocyclic planar structure of PPy. While, electropolymerization yields predominantly rough films, inhibiting PPy electronic functions, chemical polymerization results in insoluble powder. Since EHL lithography requires homogeneous films for the patterning process an optimized synthesis of PPy [34] has been carried out in this study to prepare electroconductive PPy soluble in organic solvents which can be spin-coated into homogeneous films. This process (described in the experimental section) yielded $71 \%$ of pure PPy soluble in DMF, THF, $m$-cresol and chloroform. The conductivity of the films was measured to be $1.7 \mathrm{~S} / \mathrm{cm}$ (from Chloroform). The synthesized PPy was readily spin cast into homogeneous films both on Si wafer substrates or indium tin-oxide (ITO) glass, which serve as bottom electrodes during the patterning process. These conductive polymer films were subjected to the EHL patterning process.

\subsubsection{Principal of EHD Patterning of Thin Leaky Dielectric Films}

The physical mechanism of the EHD pattern formation process in the case of a perfect dielectric is well-understood. [131, 169, 175] For EHD patterning of conductive polymers however, free charges in the film (which substantially modify the electric field distribution in the film-air double layer) have to be taken into the consideration. The 
following discussion is based on the formalism proposed by Pease and Russel [106, 107] for charge-driven EHD patterning of leaky dielectric films.

The pattern selection of EHL instabilities is given in terms of a linear stability analysis for an incompressible, Newtonian fluid assuming the non-slip boundary condition at the substrate (Fig. 8.1A). The destabilising electrostatic pressure, $p$ scales with the square of an applied voltage, $U$

$$
p=-\frac{1}{2} \frac{\varepsilon^{\mathrm{g}} \varepsilon_{0} U^{2}}{\left(d-h_{0}\right)^{2}}
$$

with the capacitor electrode spacing, $d$, the dielectric permittivity of free space, $\varepsilon_{0}$ and the dielectric constant of the gap fluid $\varepsilon^{g}$ (which is an air gap in our case, i.e., $\varepsilon^{\mathrm{g}}=\varepsilon_{1}$ ). The conductivity of the leaky dielectric suppresses the electric field in the film and the field in the gap drives the EHL pattern formation. This yields the dimensionless conductivity, $\Sigma$ representing the ratio of a time scale for free charge conduction to the process time scale

$$
\Sigma=\frac{\sigma \eta \gamma d^{3}}{\left(\varepsilon_{1}\right)^{3} \varepsilon_{0}^{3} U^{4}}
$$

where, $\gamma$ is a surface tension, $\sigma$ is a conductivity and $\eta$ is the viscosity of the polymer. In the limit of $\Sigma \gg 1$ (in our study $\Sigma$ is on the order of $10^{7}$ ) the most dominant wavelength

$$
\lambda=\frac{(2 \sqrt{2}) \pi}{U} \sqrt{\frac{\gamma\left(d-h_{0}\right)^{3}}{\varepsilon_{0}}}
$$

is given by a force balance between the destabilizing electrostatic pressure due to the field in the gap acting on the polymer-air interface with respect to the interfacial height, $h_{0}$ and the $\gamma$ acting to minimise the surface area, thus suppress the height variations. The characteristic spacing $(\lambda)$ and the characteristic time constant for the instability

$$
\tau=\frac{12 \gamma \eta\left(d-h_{o}\right)^{6}}{U^{4} h_{0}^{3} \varepsilon_{0}^{2}}
$$

are predicted to be shorter for fully conducting materials compared to a prefect dielectric, paving the way for smaller size-scale pattern sizes, greater aspect ratios and faster growing modes. [107] 

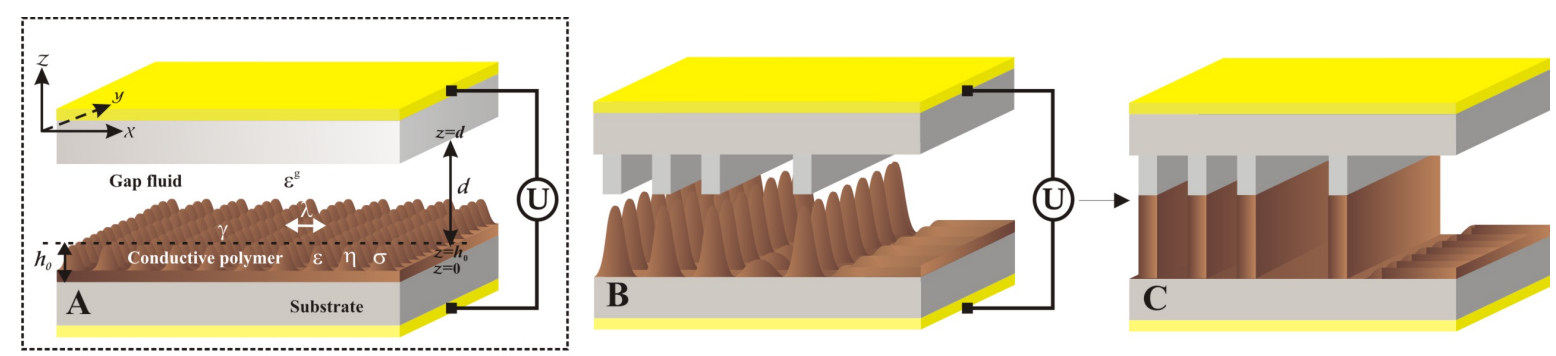

Figure 8.1: Schematical representation of electrically driven patterning set-up. (A). A thin liquefied confined film develops characteristic undulations at $z=h_{0}$ while a constant voltage, $U$ is applied to between the electrodes (or across the the capacitor gap). Contact potentials at each interface give rise to the electric field that drives the flow. The dielectric constants of the film, $\varepsilon$ and the gap $\varepsilon^{g}$ mediate the electrical forces from the applied voltage. The electrostatic force is balanced by surface tension, $\gamma$ yielding the characteristic spacing, $\lambda$ of the instability with viscosity $\eta$. (B). Heterogeneous force field directs the instability towards the protruding line structures and generates columnar-bridges between the two electrodes, followed by a coarsening of the pillars yielding in a positive replica of the master pattern in $(\mathrm{C})$. In unstructured regions, the film remains stable on a much longer timer scale.

\subsubsection{EHL Generated Conductive Patterns Exhibit a Range of Feature Sizes}

The EHL patterning process of line structures is illustrated in Fig. 8.1A-C. A topographically structured electrode induces an inhomogeneous electric field in the capacitor gap. Initial instabilities are coupled to the lateral field variation and further focussed in the direction of the highest electrostatic force. They are thus driven towards the downward protruding structures of the top mask. This results in pillars pattern spanning the capacitor gap at the locations of the smallest inter-electrode distances (Fig. 8.1B), which eventually, by coalescence generates a replicated pattern of the upper electrode (Fig. 8.1C). The final height of the polymer structures is dictated by $d$ and $h_{0}$.

Figure 8.2 shows optical microscopy (left) and atomic force microscopy (AFM) (right) images of lines and columns obtained after the application of voltage to the capacitor device. Optical micrographs reveal large areas comprised of conductive PPy patterns exhibiting a range of feature sizes (Fig. 8.2A-C, left). The cross-sectional AFM image in Fig. 8.2B reveals a line height of $2.5 \mu \mathrm{m}$, and a width of $7.5 \mu \mathrm{m}$. The length scale of the EHL formed patterns is dependent on the feature size of the structured electrode. A reduction in the topographically induced feature size and an increased electrical potential (of $75 \mathrm{~V}$ ) results in PPy lines as small as $300 \mathrm{~nm}$ height and $750 \mathrm{~nm}$ width (Fig. 8.2A). An additional pattern of ordered columns has been generated using EHL patterning after the application of $55 \mathrm{~V}$ between the electrodes. This has resulted in a columnar structure with a typical height of $330 \mathrm{~nm}$, a diameter of $1.2 \mu \mathrm{m}$ and a periodicity of $2 \mu \mathrm{m}$ (Fig. $8.2 \mathrm{C}$ ). Apart from isolated defects, the patterns of the master 


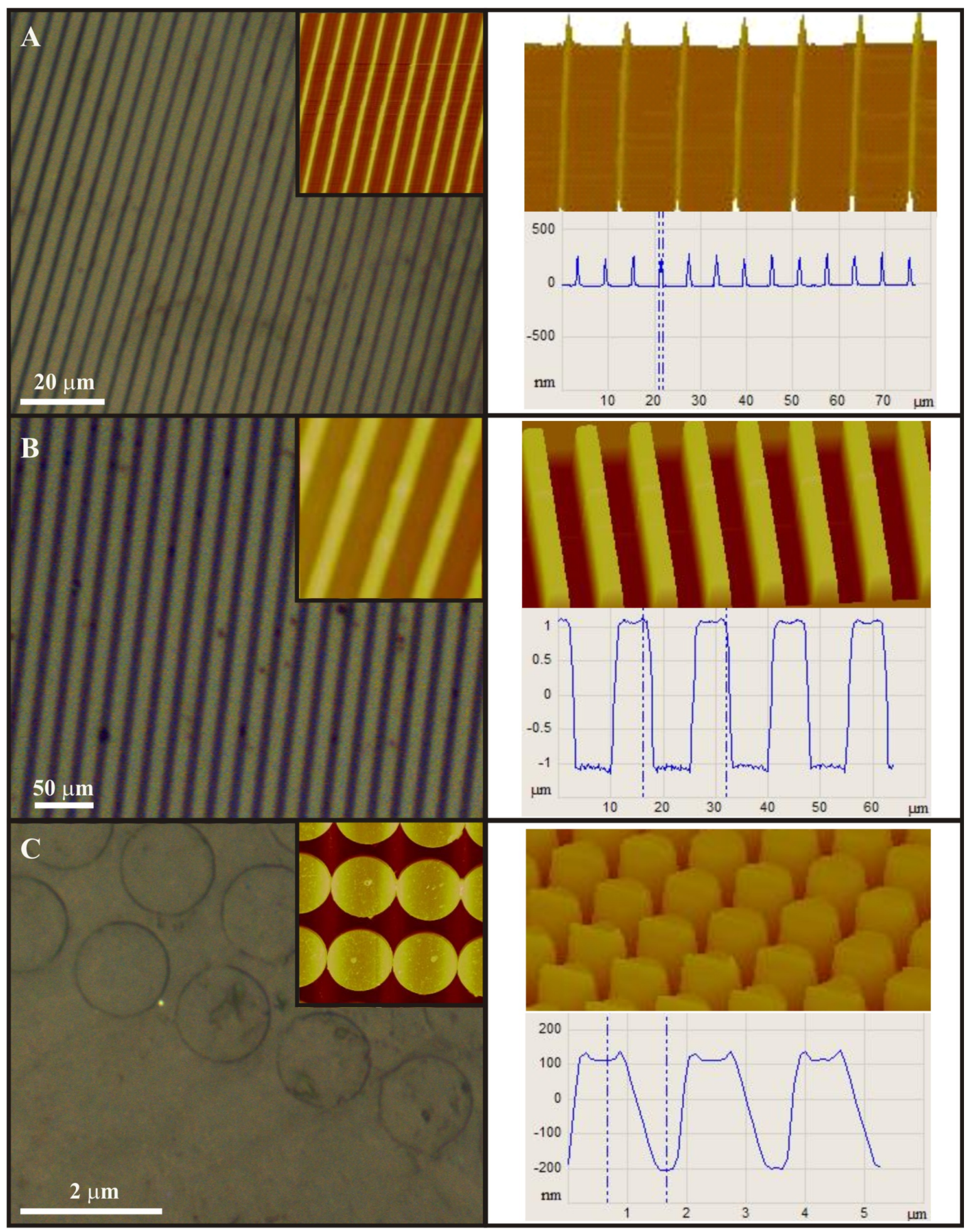

Figure 8.2: EHL replication of line and columnar patterns. Optical microscopy images (left hand side) with a height AFM image (inset) and three-dimensional AFM micrographs with cross-section analysis (right hand side) showing fabricated PPy structures: (A) $750 \mathrm{~nm}$ wide nanolines with a height of $300 \mathrm{~nm}$; (C) sub-micrometer pillars with $300 \mathrm{~nm}$ in height, $1.2 \mu \mathrm{m}$ in diameter and with a spacing of $2 \mu \mathrm{m} ; 7.5 \mu \mathrm{m}$ wide and $2.5 \mu \mathrm{m}$ height microlines (B). 
electrodes were reproduced with high fidelity over the entire electrode area (typically is $\left.100 \times 100 \mu \mathrm{m}^{2}\right)$.

\subsubsection{Tuning the Structural Morphology}

The final pattern morphology and its dimensions can be tuned by the specific set of sample parameters. The pattern formation rate, the height and the lateral dimensions (i.e., structures width and spacing, and therefore, the aspect ratio) of the EHL generated patterns can be controlled via adjustment of the applied voltage (increasing $U$ causes higher driving forces), the initial film thickness, the inter-electrode gap, the field strength and the surface tension. Moreover, the imposed modulation periodicity of the structured electrode, for which the amplitude can be adapted, not only plays an important role in obtaining a faithful replication, but also, opens up a route towards decreasing the patterns length scale to the sub-100 nm range. In the presence of the laterally varying confinement, the liquid morphologies are organized according to the ratio of the plate spacing and the initial amount of polymer in the capacitor gap. While the pattern selection during the early stage of the process is a sinusoidal surface undulation, for all samples, filling ratio establishes the late stage of pattern formation. The final morphology of the replicated pattern is determined by the partial coalescence of the initial pattern.

The shape of the generated pattern depends on the ratio of the intrinsic film undulation wavelength and the lateral periodicity of the master electrode. Thus, sub-100 nm patterns with structure-to-structure spacing on the order of $100 \mathrm{~nm}$ can be achieved with a leaky dielectric by both exploiting conducting polymer as film material and gap materials that combine a low interfacial tension with the film with a high electric strength.

\subsection{Conclusions}

In conclusion, a conducting polymer EHL patterning process is directly performed on silicon substrates yielding high fidelity structures with a range of feature scale sizes. While patterning of PPy is a proof-of-concept, the versatile EHL patterning method can be can be applied to variety of CPs. With properly chosen experimental parameters combined with an adequate master electrode, it should be possible to generate patterns with sub-100 nm widths over very large areas. Submicrometer structured conducting polymers possess improved properties and performance compared with the bulk material devices. These structures therefore, have potential applications for the development of microelectronics, displays and biotechnological assemblies, such as flexible display 
devices or bio-and chemical sensors. For example, high density electrically conducting microstructures can be directly used as miniaturized sensors. The EHL approach can be further extended to lateral complex or hierarchical structures consisting of bilyers or larger numbers of different materials with set mismatched conductivities.

This is the first time that conductive polymer has been patterned using EHL technique. This method provides promising route for a straightforward and cost-effective large area patterning of CPs, opening up many opportunities for the high resolution and throughput structures with applications in nano and bio-technology related fields and devices. The current research focuses on the EHL generated electrically conductive microstructures of PPy which can be used directly as arrays of electrodes to fabricate all plastic FETs. 


\section{Structural Hierarchy of Functional Block Copolymer System Induced by}

Electrohydrodynamic Lithography

The advantages arising from anisotropic functional block copolymer nanostructures hold great promise for applications in electro-optical devices made of organic materials. Efficient hierarchical structures are formed exhibiting order on several length scales by using electrohydrodynamic patterning approach. Initially block copolymer microdomains oriented parallel to the substrate are destabilized by electric fields yielding formation of microstructures perpendicular to the substrate. Within each fabricated microstructures the cylinders are aligned parallel with respect to the electric field lines. An internal vertical alignment of the copolymers nanometric domains exhibits an additional molecular orientation thus, providing a straightforward path for exciton transport to the donor-acceptor interface essential for the enhanced charge generations. Inside the superstructured films, ordered arrays of nanocrystals of the constituting block are aligned in smectic phase giving rise to linear dichroism. Controlled alignment of the nanodomians improves the charge conduction toward the device electrodes. These findings, while establishing a straightforward strategy to produce oriented assemblies of nanodomains of electroactive polymers, open a new avenue for nanotechnologal applications and are very promising for optoelectronic applications, especially in photovoltaic devices.

\subsection{Introduction}

The ability to pattern functional polymers at different length scales is important for a range of applications and developments including the fabrication of micro-and plasticelectronics, the production of optical components and bio-medicinal related research. Self-assembly of block copolymer (BCP) films arsing from the microphase separation into the periodically ordered topographic nanopatterns has been evolving as an attrac- 
tive means for the fabrication of mesoscopic devices based on electronic and photonic materials. [11, 36] The intense interest in block copolymers for many nanotechnological applications originates from the achievable length scales ranging from 10 to $100 \mathrm{~nm}$ and the ability to tailor their chemical structure for desired functionality, providing the addressability of self-assembled nanodevices. Thus, BCPs have found uses in microelectronics, solar cells, [22, 144] lithographic techniques, [8] storage devices, [157] organic semiconductor applications, [105] molecular sieves [20] etc. Of a particular interest, are perpendicularly oriented block copolymer domains with a large interfacial area separating well-defined percolating pathways, continuously spanning from the top to the bottom of the film. For instance, vertically oriented microdomains in semiconducting polymers (i.e., comprised of donor-hole transporting and acceptor-electron transporting blocks) hold great promise for the application in organic photovoltaics. The construction of vertically oriented nanodomains in donor-acceptor BCPs, percolating across the device might provide a solution for combining charge transport with sufficient optical absorption on a length scale commensurate with the exciton diffusion length. [11, 145] Although block copolymer self assembly is a highly efficient process, in thin films it typically results in an orientation of the microdomains parallel to the substrate due to the surface interactions. Significant advancement has therefore been made in the alignment of the BCP nanostructures. The control over the orientation of block copolymers domains was demonstrated using mechanical shear forces, [163] temperature gradients, [17] epitaxy, [136] electric fields [156, 158] and annealing in a solvent vapour. [68, 69] The optimal thin film morphologies directly suitable for a range of implementations have therefore been recognized. The feasibility of electrically induced pattern formation with structural control over the block copolymer microphase morphology has been investigated for amorphous dielectric copolymers. [167, 179] However, the successful implementation of self-assembled BCPs-based devices with the simultaneous control over the assembly and positioning of the oriented block copolymer domains into desired locations to construct complex, functional structures still remains a grand challenge. To date, no functional device directly obtained via orientation and controlled modification of self-assembled diblock copolymers has been demonstrated.

Here, we describe the fabrication of hierarchical functional patterns using electrohydrodynamic instabilities to pattern a fully functionalized electron-conducting block copolymer. Our method combines the bottom-up self-assembly of block copolymer films (with internal structures on a scale of some tens of nanometres) with the induced self-organization of the intermolecular structure of the constituent crystalline blocks (in the range of 2-3 nm) inside the EHD driven microstructures, to produce functional patterns with a structural hierarchy. The formation of patterns spanning several length scales is primarily understood in terms of the interplay of molecular self-assembly and 
the structural control exerted by electrostatic forces. The driving torque for both the electrically induced copolymer domain orientation and the EHD driven instability is the polarization of dielectric interfaces. Block copolymer microstructures respond to electric fields in a similar manner as the EHD induced instability, by coupling to the dielectric contrast between the different domains. The destabilizing electrostatic pressure resulting from the electric field causes the energetically unfavourable build-up of displacement charges at the dielectric interface causing the alignment of the interface (of two chemically different blocks, or of polymer-air free surface) parallel to the electric field lines, lowering the electrostatic energy. Amplification of the interfacial fluctuations caused by the interplay of the destabilizing electrostatic pressure and the restoring surface tension yields eventually a pattern of polymer pillars, or in the case of topographically patterned electrodes, a positive replica of the imposed structures. These micrometer-sized patterns have an additional sub-nanostructure arising from the microphase-separated BCP morphology aligned parallel to the electric vector. Oriented nanodomains constitute an additional degree of order on the sub-10 nm scale consisting of the BCP crystalline stacks with the $\pi-\pi$ interactions oriented perpendicular to the electric field direction. These electro-conductive nano-domains within the pillars act as half-wave plate polarisers. The resulting morphology represents the optimal orientation of the copolymers nanostructure with respect to the substrate, exhibiting enhanced charge carrier percolation between the two electrodes. The charge transport across the entire device renders these aligned nanomorphologies appealing for a range of technological applications, in particular for organic photovoltaic cells.

\section{$9.2 \quad$ Experimental}

Polystyrene-block-poly(perylene bismide acrylate) (PS-b-PPerAcr) block copolymer with a $70 \mathrm{wt} \%$ fraction of PPerAcr and $M_{\mathrm{w}}$ of $38 \mathrm{~kg} / \mathrm{mol}$ was used. In this polymer, perylene bismide (BPI) units are attached to a polyacrylate backbone. PBI is a well-known electron acceptor and electron transporting material with the $\pi-\pi$ interactions, which lead to side chain crystallinity of PPerAcr. (Fig. 9.4b, inset) This polymer self-assembles into a cylindrical microphase morphology in the bulk. [145] Highly polished $p$-doped silicon (Si) wafers, with $<100>$ crystal orientation (Wafernet Gmbh, Eching, Germany) were used as substrates. Patterned silicon wafers were obtained from X-lith eXtreme Lithography, Ulm, Germany). Indium tin oxide (ITO)-coated glass slides with a resistance of $112 \Omega / \mathrm{cm}^{2}$ were also used as transparent electrodes. Thin block copolymer films were spin cast from chloroform solutions resulting in typical film thicknesses between $50 \mathrm{~nm}$ to $300 \mathrm{~nm}$. Prior to spin-coating, the substrates were cleaned in a 'Piranha' solution consisting of 3:1 $\mathrm{H}_{2} \mathrm{SO}_{4}(98 \%): \mathrm{H}_{2} \mathrm{O}_{2}(30 \%)$, followed by 
thorough rinsing with deionised water and drying under nitrogen. The ITO-coated glass slides were cleaned by scrubbing in soap water at $75^{\circ} \mathrm{C}$, washing in an ultrasonic bath with acetone and isopropanol, followed by irradiation for 20-30 minutes in an UVozone cleaner. Immediately before device assembly, all substrates and electrodes were subjected to snow-jet cleaning. Facing the coated substrate, a wafer with a planar or topographically structured surface, was mounted at specific distance using photolithographically prepared spacers, leaving a thin air gap, $d$ (Fig. 9.1b). Both capacitor electrodes were electrically contacted using silver paint (Electrodag 1415M). Thermally evaporated $50 \mathrm{~nm}$ thick $\mathrm{Au}$ layers on the reverse side of the electrodes ensured good electrical contacts.

The spin cast films were liquefied by either annealing above the softening temperatures of the two blocks $\left(T=75^{\circ} \mathrm{C}\right)$ or by exposing them to a controlled vapour atmosphere of chloroform to induce chain mobility and facilitate equilibration. The solvent-vapour pressure was adjusted using a home made apparatus. [165] The films were allowed to swell in the controlled solvent vapour atmosphere to their equilibrium thickness (around 30 mins [165]). A voltage of $55-80 \mathrm{~V}$ was applied between the two electrodes. In the case of temperature annealing, cooling down the sample to RT, or removal of the solvent by passing dry nitrogen through the sample chamber in the case of solvent annealing and removing the electric field terminated the patterning process. After each experiment, the top electrode was removed and the formed pattern was analyzed by light microscopy (LM), atomic force microscopy (AFM), scanning and transmission electron microscopy (SEM and TEM) and the combination of the latter (STEM).

NanoScope IV Multimode and Dimension 3100 (Digital Instruments, Santa Barbara, CA) atomic force microscopes were employed to quantitatively determine the sample topography. All the measurements were performed in air under ambient conditions using the tapping mode. Height and phase images were analyzed with the Nanoscope software (Digital Instruments). The AFM measurements yielded the film thickness $h$, the plate spacing $d$, the characteristic wavelength $\lambda$, and the width and height of the replicated patterns. To improve contrast, patterns were exposed to UV-light and rinsed in cyclohexane to remove the PS phase. A LEO ULTRA 55 SEM microscope including a Schottky emitter ( $\mathrm{ZrO} / \mathrm{W}$ cathode) was used to reveal the cylindrical block copolymer morphology. For TEM cross sectional imaging, a thin layer of Pt was sputtered onto the block copolymer films, followed by embedding into a Spurr epoxy resin. The substrate was removed and the films were sectioned using diamond knife in a Leica Ultracut Microtome, yielding sections with a thickness of $50 \mathrm{~nm}$. The cross-sections were analyzed using a FEI Technai 12 transmission electron microscope (TEM) at acceleration voltages of 80 or $120 \mathrm{kV}$. The microtomed samples were stained with ruthenium 
tetroxide vapors to improve the contrast. STEM images were obtained using Hitachi s5500 scanning transmission electron microscope with a cold field emission source and lens detector with 4 Angstrom resolution, allowing adjustable acceptance angle STEM imaging. An Olympus BX51 optical polarizing microscope was used to investigate the optical textures of the samples. Optical anisotropy of oriented samples was evaluated with regard to variations of the intensity of reflected or transmitted light on a LM with a spectrometer attached (QE6500, Ocean Optics). The microscope halogen lamp acted as an illumination source for the spectroscopic measurements. Linear polarization measurements were obtained using achromatic polarisers (Thorlabs, Inc.) in cross and parallel configurations. In addition, the sample was mounted on a multi-rotational stage allowing rotation around the focal axis of the objective. This configuration enabled transmission and reflection measurements to be taken in different polarisation directions with respect to the sample orientation. The maximum intensity of polarized light corresponds to the chains or molecules aligned parallel to the polarizing filter $\left(45^{\circ}\right)$ and the minimum when oriented at plus or minus $45^{\circ}$ with respect to the filter $\left(0^{\circ}\right.$ and $\left.90^{\circ}\right)$. Current-voltage $(J-V)$ measurements were carried out using Kiethley 2400 source-meter in air. The top ITO electrode consisted of a small strip ITO on a glass slide to selectively cover the area of interest for $J-V$ measurements. Both top and bottom ITO electrodes were photo-lithographically patterned to reduce possible leakage currents.

\subsection{Results and Discussion}

For the EHD patterning process we exploited the electron transporting diblock copolymer polystyrene-block-poly(perylene bismide acrylate) (PS- $b$-PPerAcr) with a molecular weight of $38 \mathrm{~kg} / \mathrm{mol}$ containing $70 \mathrm{wt} \%$ PPerAcr, targeting the cylindrical morphology (Fig. 9.1a). This BCP has been synthesized and extensively analyzed because of its high electron carrier mobilities. [60, 145] Thin films were spin-coated from chloroform solutions onto silicon substrates. This resulted in a lying cylindrical microphase morphology parallel to the substrate, schematically shown in Fig. 9.1b. This morphology was confirmed by scanning electron microscopy (SEM) in combination with cross sectional transmission electron microscopy (TEM) shown in Fig. 9.1c-d. Cylinder orientation parallel to the substrates is often observed, arising most probably from the selective BCP interaction between the two blocks and also between the confining substrate. These films were further confined between two electrodes with a small air gap, and annealed above the glass transition $\left(T_{\mathrm{g}}\right)$ of PS and the melting temperature $\left(T_{\mathrm{m}}\right)$ of PPerAcr $\left(100^{\circ} \mathrm{C}\right.$ and $189^{\circ} \mathrm{C}$, respectively) or alternatively, annealed in a solventvapour atmosphere, where the film swells by solvent take-up, causing an increase in 
polymer-chain mobility similar to temperature annealing above $T_{\mathrm{g}}$. Subsequently, a voltage was applied perpendicular to the film. The experimental set-up is shown in Fig. 9.1b.

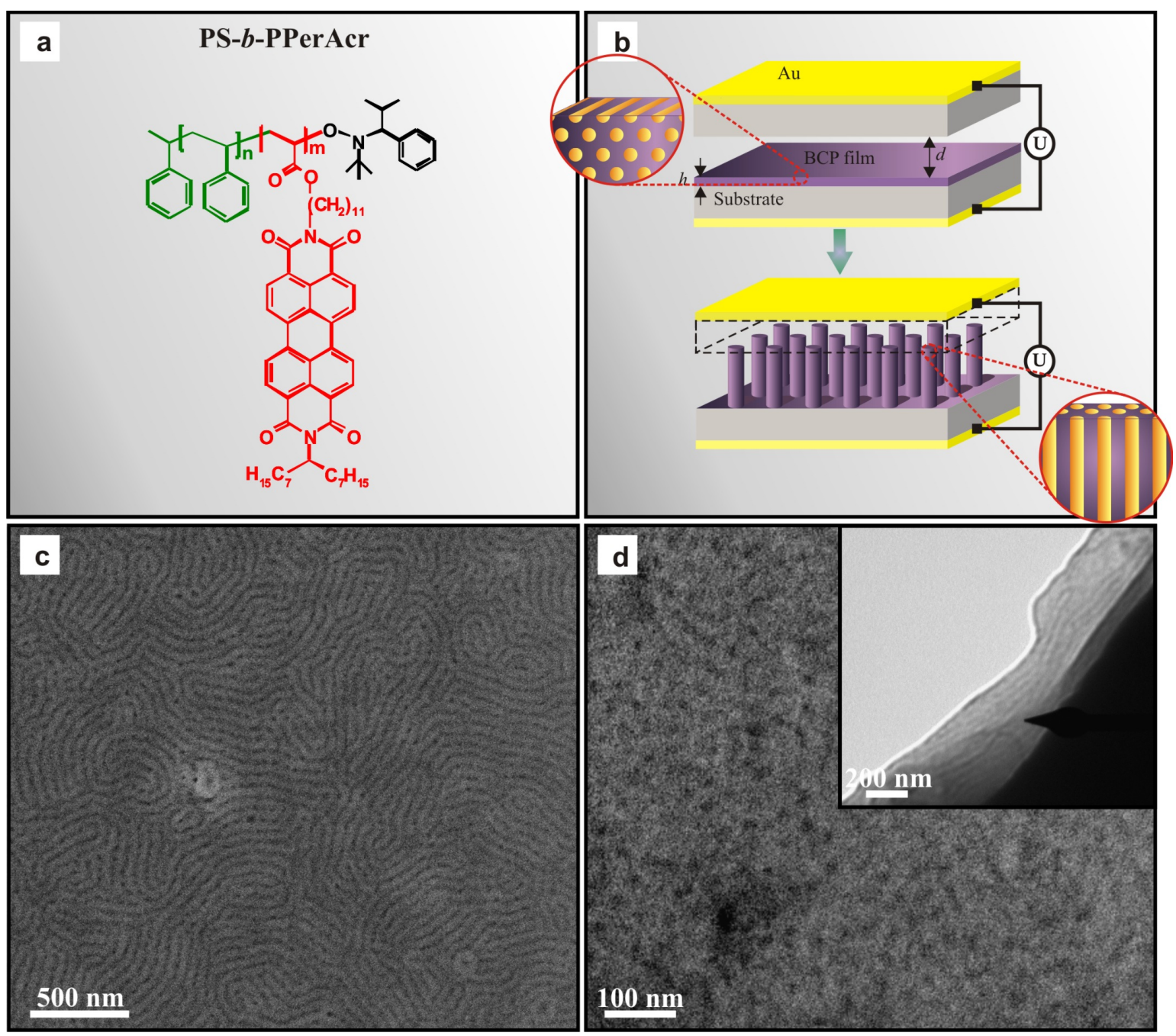

Figure 9.1: (a). Molecular structure of the PS- $b$-PPerAcr block copolymer consisting of an inert block of polystyrene and a crystalline side chain PPerAcr. The dilution with amorphous segments does not alter the charge-transport motilities. [145] (b). Schematic image describing the principle of the EHD patterning process. The thin BCP film in the capacitor is liquefied and the amplification of a capillary surface instability is triggered by applying a voltage $U$. With time the instability causes the formation of liquid bridges between the two plates. Zoomed-in images show internal structure of the BCP film before (lying cylinder) and after EHD patterning (vertical cylinders) process. (c). STEM micrograph revealing morphology of a spin-cast thin BCP film. (d). Cross-sectional TEM image perpendicular and parallel (inset) to the surface of spin cast film with a lying cylinder morphology. The images were stained with ruthenium tetroxide vapours to improve contrast.

The application of an electric field resulted in a destabilising electrostatic pressure prevailing over the restoring surface tension at the interface, giving rise to an undulation instability with a well-defined wavelength. Figure $9.2 \mathrm{a}$ reveals the pillars pattern 
resulting from a homogeneous electric field. For a topographically structured electrode, the wave pattern couples to the lateral field variation, and the further growth of the instability is focused in the direction of the highest electric field towards the protruding patterns of the top electrode. This gives rise to a pattern spanning the substrate and the top electrode at locations of smallest interelectrode spacing. A replication of design top topography induced by a laterally heterogeneous electric field is shown in Fig. 9.2b. Annealing in a solvent-vapour atmosphere in contrast to the classical thermal annealing used for EHD patterning in Figs. 9.2a-b, results in a comparable high-fidelity replica of the master electrode (Fig. 9.2c). All the obtained patterns are on the micrometer scale with typical diameters of $1-5 \mu \mathrm{m}$.
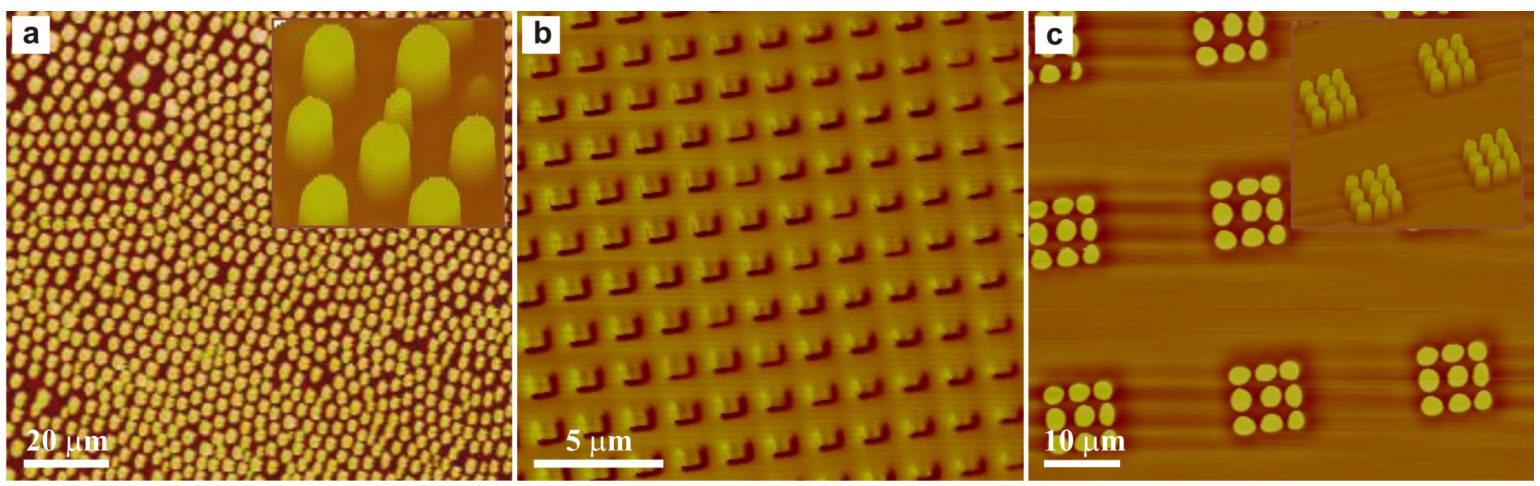

Figure 9.2: AFM height images of EHD generated pattern geometries: (a) array of pillars with local hexagonal symmetry formed in the presence of a homogeneous electric field, (b) array of squares caused by a laterally varying field, both liquefied by raising the temperature above the glass transition temperature of the material, and (c) Square arrays of pillars generated by a patterned top electrode in a solvent vapour atmosphere.

The primary EHD-generated micrometer scale patterns exhibit an additional internal substructure comprised of a well-ordered array of circular pores with an average diameter of $14 \pm 3 \mathrm{~nm}$, revealed in scanning transmission electron (STEM) micrographs (Fig. 9.3a). Note that these images were taken of the unmodified sample, without partial degradation or sputtered-on metal. The ring-like FFT pattern of the STEM image indicates that the size distribution of the pores is narrow and the presence of a weak second-order ring is indicative of the medium-range order. Vertically aligned cylindrical domains parallel to the axis of the micrometer-sized pattern (along the lines of the electric field and the material flow) extend from the free surface down to the substrate (Fig. 9.3b and d). Comparably, swelling in a solvent vapour atmosphere yields a vertical, hexagonally packed array of cylindrical domains percolating through to the substrate (Fig. 9.3d). Interestingly however, in the case of solvent-vapour annealing a higher degree of lateral order of cylindrical array is obtained (Fig. 9.3c). The six-fold symmetry of the FFT pattern indicates that the nanodomains are hexagonally 
packed (Fig. 9.3c, inset). However, the absence of second-order peak implies that the microphase oriented morphology lacks long-range order in the film. Solvent-vapour ordering can be understood in terms of a gradient in the solvent concentration front propagating through the film as proposed by Russell et al. [69] The BCP is disordered in the centre of the film where solvent concentration is higher in comparison to the film surface where the evaporation of the solvents results in the lowest concentration, producing better order in the oriented array of cylindrical microdomains in the film. In addition, EHD alignment of a BCP in a solvent-vapour atmosphere is more efficient than thermal annealing, significantly reducing the risk of thermal degradation of the electronically active material, and also resulting in a considerably shorter patterning times. 
9 Structural Hierarchy of Functional Block Copolymer System Induced by Electrohydrodynamic Lithography
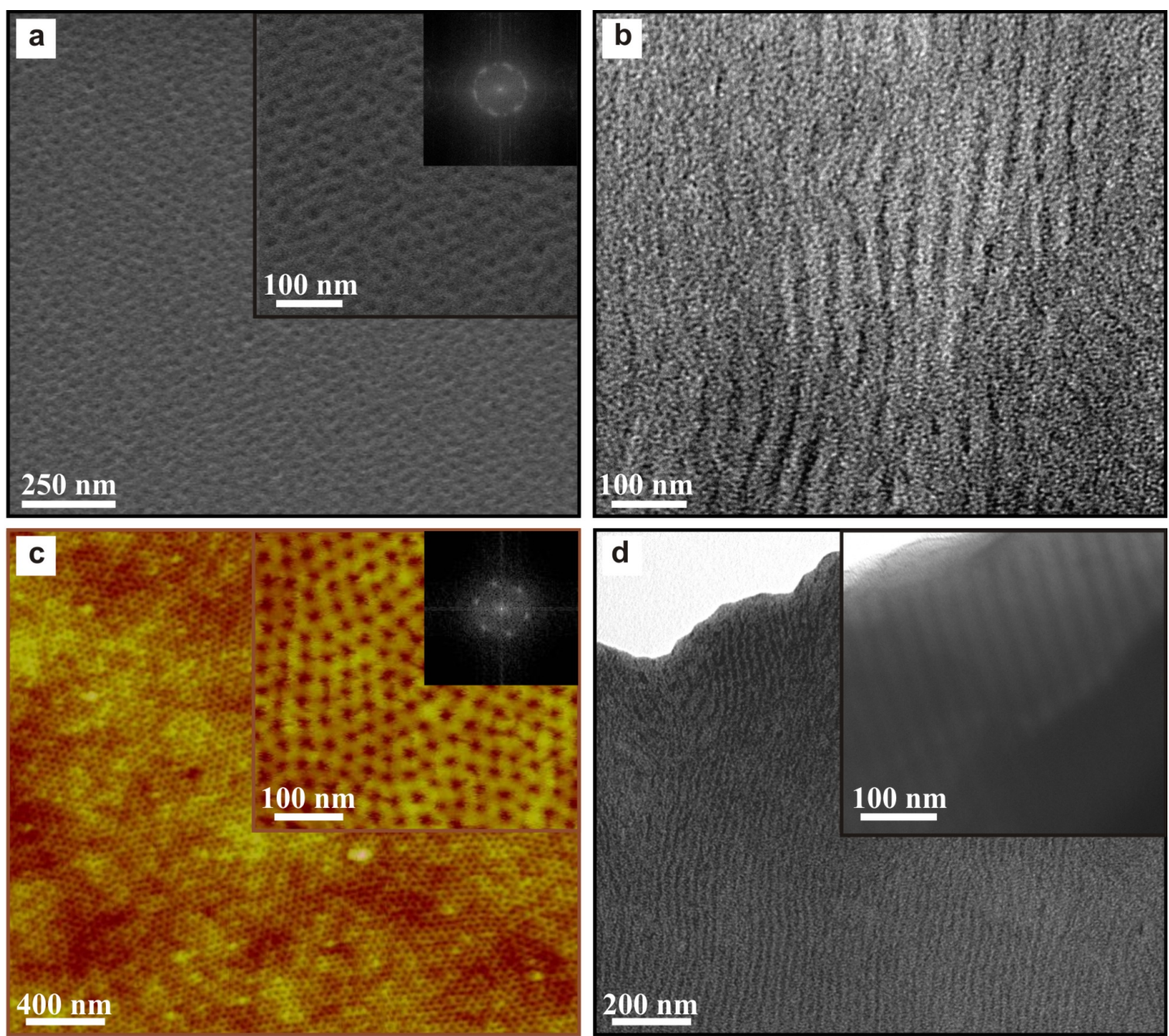

Figure 9.3: Perpendicularly oriented PS- $b$-PPerAcr microdomains that have formed inside the EHD generated structures. (a). Surface STEM image showing a porous BCP film that was thermally annealed above the softening temperature $\left(\right.$ at $215^{\circ} \mathrm{C}$ ) and subjected to an electric field (with an applied voltage of $70 \mathrm{~V}$ ) for $24 \mathrm{~h}$. Inset: ring-like FFT pattern of the STEM image. (b). Cross-sectional TEM view of (a) revealing the orientation of the cylindrical microdomains normal to the substrate. (c). Representative AFM height image shown the improved packing of cylinders with an average pore diameter of $14 \pm 3 \mathrm{~nm}$, formed inside PS-b-PPerAcr matrix when swollen in chloroform vapour with a $80 \mathrm{~V}$ applied across the capacitor electrodes for $5 \mathrm{~h}$. The inset in (c) displays a FFT pattern of the structured film with a six-fold symmetry representing hexagonal packing of the cylindrical nanodomains. (d). Cross-sectional TEM micrograph of (c) revealing the compete alignment of the cylinders spanning continuously from the top to the bottom of the patterned film.

The resulting vertical alignment of cylindrical morphology in the EHD generated primary patterns is caused by the combined effect of the flow of the material into these microstructures and the action of the electric field on the annealed BCP. The difference in the dielectric constants between the two constituting blocks provides the driving force induced by the electric field. [179] The electric field distribution is modulated during the EHD pattern evolution with the strongest field experienced by the material 
once it spans the air gap and establishes a contact with an upper electrode. The reorientation from the lying cylinders morphology to the standing cylinders occurs once the electrostatic torque coupled to the hydrodynamic convection forces of the flow surpass the competing interfacial interactions of the surface field.

Interestingly, we found that EHD induced hierarchical micropatterns of PS- $b$-PPer Acr with internal oriented nanocylinders have an additional degree of order on the sub-10 nm scale consisting of the BCP crystalline stacks with the $\pi-\pi$ interactions oriented perpendicular to the electric field direction. Figure 9.4a shows an alteration of the polarization direction of linear polarized light when travelling through the hierarchical BCP patterns. This half-wave plate like behaviour of the patterns implies that side-chain polymer crystalline stacks of PPerAcr are laterally aligned perpendicular to the pattern and the cylindrical domains (i.e., to the electric field) direction facilitating a formation of smectic-like phase. The generic (non-patterned) PS- $b$-PPerAcr microphase-segregated morphologies comprised of perylene bismide moities (PBI) result in isolated domains of liquid crystalline polymer assembled into crystalline stacks with no preferential order. EHD patterning induces preferred alignment of the $\pi-\pi$ stacking direction perpendicular to the plane of the $2 \mathrm{D}$ lattice of the stacks. The presence of a vertically aligned nanodomain morphology in this system provides an environment with modulated interfaces between the two blocks of the liquid crystalline polymer, in which an amorphous PS block is assembled into a random coil directing the orientation of crystalline stacks of PPerAcr into a well-defined order (Fig. 9.4b). The PS- $b$-PPerAcr used in this study is different from classical amorphous-amorphous BCPs due to the presence of the side chain crystalline PPerAcr block. Such a system is comparable to to rod-coil block copolymers. [51] Constraining such a molecular structure into vertically oriented nanocylinder domains leads to a rearrangement of the blocks on the molecular level into a favourable configuration, aligned along a preferred direction.

The formation of a smectic phase has been modelled for hard rod fluids with both translational and orientational freedom showing a thermodynamically stable smectic phase. [40] In addition, it was shown that the attractive dipole-dipole interaction between adjacent nanorods can lead to anti-parallel side-by-side pairing of dipole moments and facilitate the formation of smectic phases. [6] 

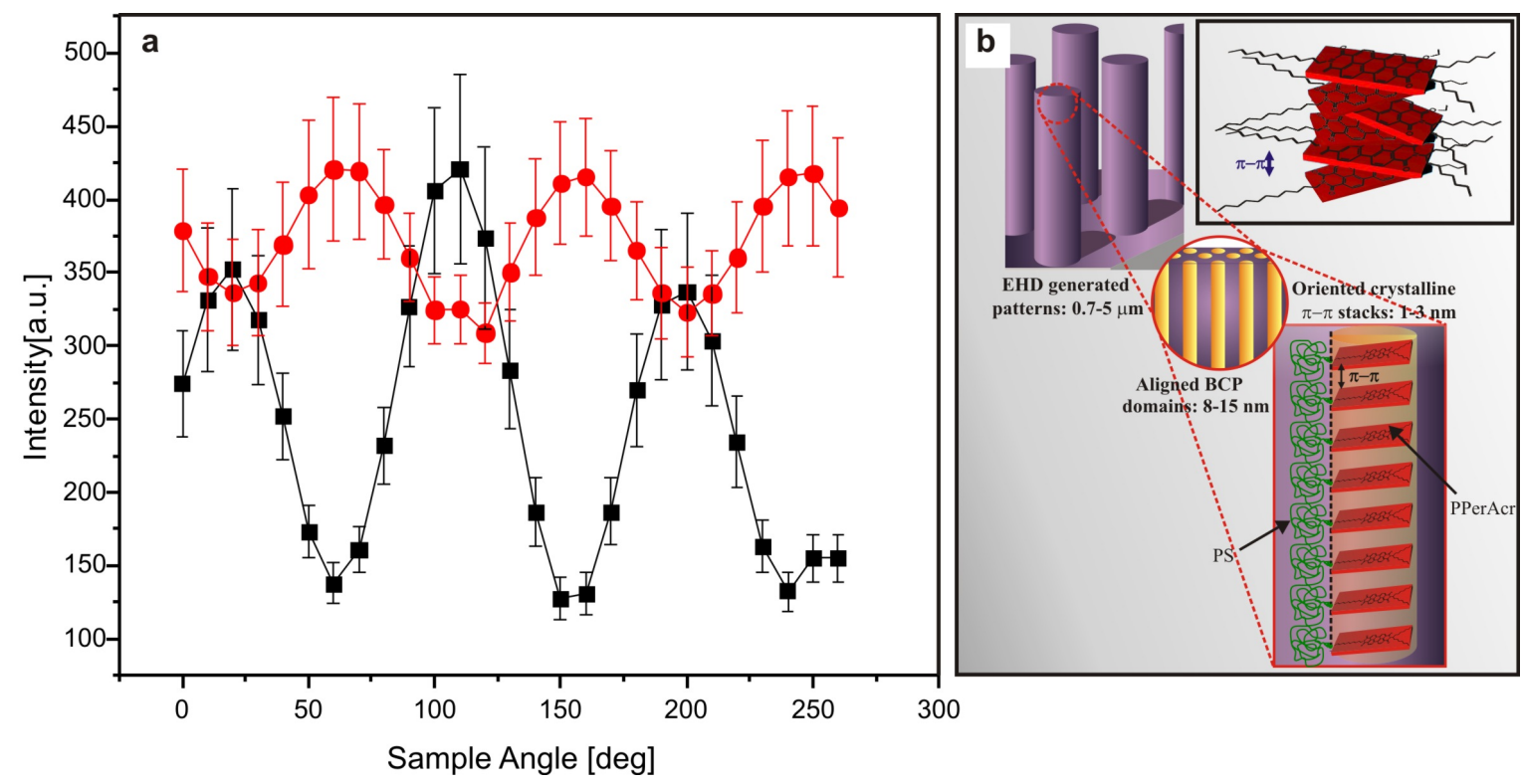

Figure 9.4: (a). Optical anisotropy analysis of internally oriented PS- $b$-PPerAcr patterns. Intensity dependence on the sample angle in a cross (black squares) and parallel (red circles) polarization configuration. (b). Schematic image of the structural hierarchy. The micrometer-sized EHD generated PS- $b$-PPerAcr patterns are comprised of vertically fully aligned nanoylindical domains (tens of $\mathrm{nm}$ ) and an additional internal nm-order within the cylinders consisting of the crystalline PPerAcr stacks. The $\pi-\pi$ stacking direction perpendicular to the plane of the 2D lattice is induced by the modulated interface between the two blocks inside the aligned cylinders.

The unique situation in which PPerAcr moieties are closely packed and oriented within block copolymer domains can provide additional advantages for the electron transport. Higher electron mobility is expected due to the strong intermolecular interaction between adjacent PBI moieties bound to the polymer backbone, leading to side chain crystallinity. The electrical properties of the BCP patterned pillar structures were further examined to show their potential use in optoelectronic applications. To determine the effect of patterned polymer pillars on charge transport we measured three types of sandwich geometries using a Kiethley 2400 source measurement unit. These devices varied in terms of different processing conditions of the BCP, schematically shown in Figure 9.5. A symmetric J-V response was obtained for all the samples.

The current density was lowest for the as cast flat films, two orders of magnitude higher for EHD patterns in the thermal annealing case, which further increased by a factor of three in the case of patterned structures of BCP in a solvent-vapour environment. Higher degree of order inn the oriented arrays of cylindrical microdomains in the film along with possible solvent residues in the film are believed to be the reason for the higher current densities in the swollen films. Although the current density for patterned devices was calculated using an entire coverage area of patterned film, there are voids in between vertical cylinders of BCP through which no current was passing. 
Hence, absolute current density for patterned film is expected to exhibit further increase upon normalization of the current per correct area (i.e., area percentage covered by cylinders in $2 \mathrm{D}$ plane can be calculated by diameter and gap in between cylinders) of polymer touching to top contact electrode. Because of the small patterning area and very thin BCP layer leakage currents are possible caused by the inherent ITO roughness. Nevertheless, a clear trend in current magnitudes caused by the different processing conditions can be seen in Fig. 9.5. This functional polymer has been previously used for heterojunction solar cells with different donor systems. [87, 144, 145] The approach presented herein provides a well-defined charge transport network for organic solar cells possibly giving rise to improved efficiencies. Current research work is therefore focussed on the exploitation of the described patterning approach to obtain working photovoltaic devices based on nanostructured bulk heterojunction that provide bicontinuous percolation of charges giving rise to enhanced device efficiencies.

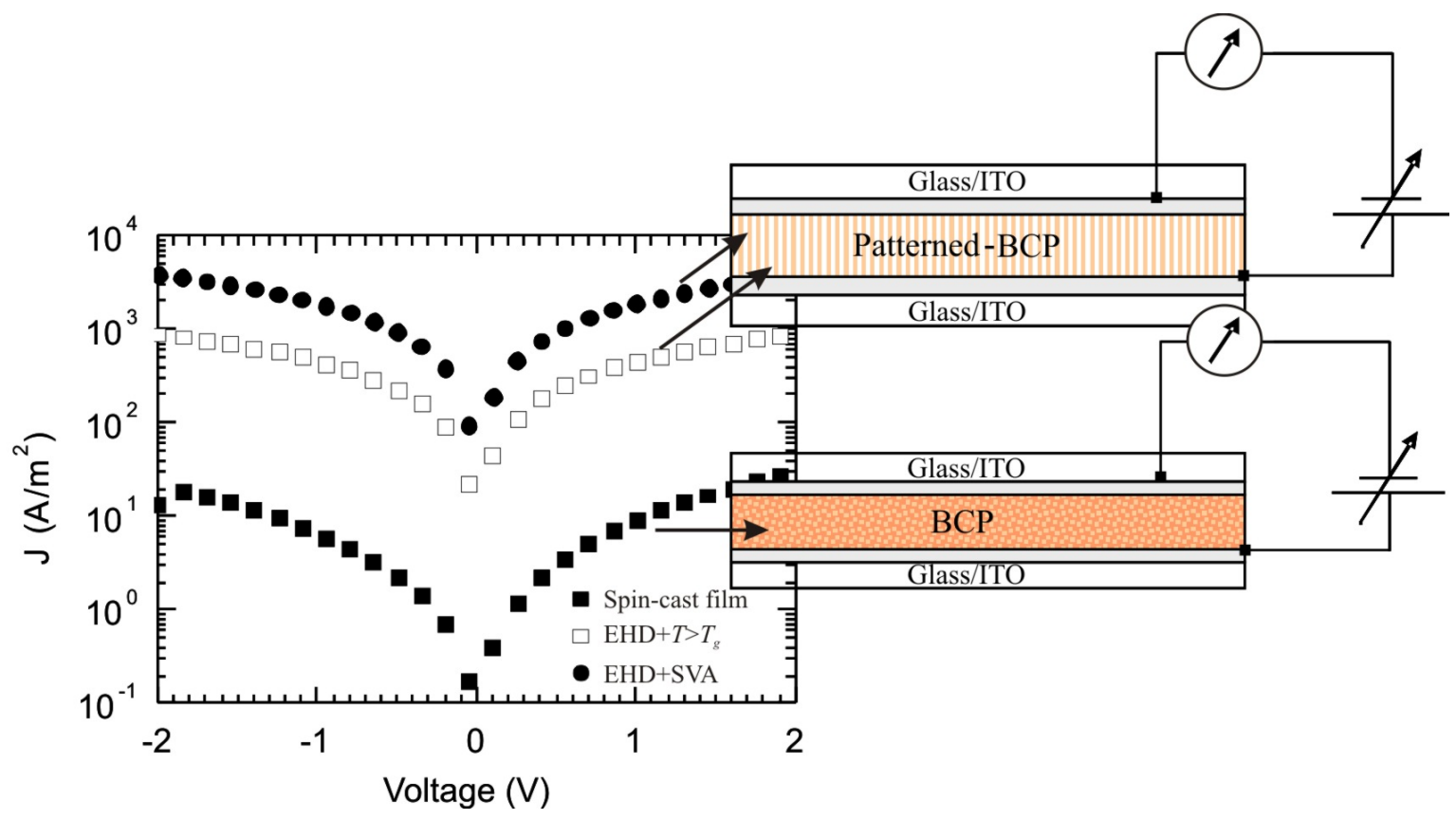

Figure 9.5: J-V characteristics (DC) of ITO/BCP/ITO sandwiches non-annealed (black squares), EHD patterned using thermal annealing (white squares) and patterned in a solvent-vapour atmosphere (black circles) show improved charge transport properties in the patterned BCP films. The side panel shows the device setup used for these measurements.

Such hierarchical structures with a high-degree of order spanning several lengths scales open up exciting opportunities into the design of more efficient and advanced organic devices. Smectic hierarchical superstructured films exhibiting polarizing characteristics can be also integrated as an emissive layer into hybrid organic/inorganic organic light-emitting diode (OLED) devices to produce polarized electroluminescence. This technique can be further used for micro-patterned polarized electroluminescent 
display devices with low operating voltages. Furthermore, while BCPs without electronic functions are primarily used for lithographic templates and scaffolds, fully functionalized electro active donor-acceptor BCPs, enable their direct exploitation, eliminating the need for further templating or multistep lithography. Patterning of functional polymer systems into addressable high-density arrays of anisotropic nanoscopic elements using a straightforward, cost-effective EHD lithographic method might enable various applications, including, for example, photonic band gap materials, sensors, nano-membranes, micro-opto-electro-mechanical systems (MOEMS) and lithographic fabrication of higher-order nanostructures.

\subsection{Conclusions}

We have demonstrated a straightforward method to fabricate ordered hierarchical structures spanning several length scales from a functional electro active block copolymer. Electrohydrodynamic lithography in particular, when combined with solventvapour annealing yields variety of micrometer structures with a well-ordered hexagonally packed array of cylinders aligned perpendicularly to substrate, parallel to the electric filed vector. Most remarkably, this gives rise to a high degree of order within the BCP domains, which considerably influences the charge-carrier mobility. A continuous interdigitation of donor-acceptor phases on the $10 \mathrm{~nm}$ length scale is established while maintaining optimal charge transport pathways percolating between the external electrodes. The geometric confinement of the functional units in the vertically oriented nanodomians has further interesting consequences on the $\pi-\pi$ interactions within the crystalline block. Crystalline stacks on the scale length of single $\mathrm{nm}$ are laterally aligned perpendicular to the pattern and the cylindrical domains (i.e., to the electric field) facilitating the formation of smectic-like phase, and giving rise to birefringence.

The described principle employs block copolymer self-assembly for the controlled formation of $10 \mathrm{~nm}$ sized patterns with crystalline order on the nm-scale, interfaced by larger structures, providing addressability of self-assembled nanodevices. Functional block copolymers containing side chain liquid crystalline segments patterned in this manner may provide a new means of combining the advantages of traditional polymeric systems with liquid crystalline electro-optical properties. It is also anticipated that many of these materials may exhibit other interesting properties, such as mechanooptical and piezoelectric behavior. The findings presented will provide a significant impact on the development of novel self-assembled organic electronic devices in the future. 


\section{Summary}

The research presented in this thesis illustrates the many interesting aspects of instabilities in thin liquid films which are both of scientific and technological importance. While thin film instabilities are typically undesired in industrial and technological applications, they lead to novel surface morphologies and are applicable in alternative (soft-)lithographic techniques. In this context, this work exploits instabilities in thin liquified films stemming from externally applied electrostatic forces to hydrodynamically generate topographic architectures with a controllable length scale in the technologically interesting sub-micrometer and sub-100 $\mathrm{nm}$ range.

In the absence of any external force, the film evolves under the influence of the long-ranged van der Waals forces causing a disjoining pressure. Van der Waals interactions are predominantly caused by electromagnetic vacuum fluctuations and the resulting induced dipole-dipole interactions. In addition to the electromagnetic fluctuations, there are always thermal vibrations present. Both of these forces, however, are comparatively weak. An externally applied field, can generate forces orders of magnitude larger, which thus dominate film destabilization. In such a limit, the instability can be attributed clearly to the applied field. In order to apply an external field, a counter electrode is placed at a certain distance from the substrate, which supports the film. The result is a capacitor-like device comprised of a polymer film and an air gap between two flat substrates, kept apart by spacers controlling the distance. Applying an electrical potential to this set-up results in an electric field. The polarization of the polymer-air interface causes a pressure difference across it, which yields an electrohydrodynamic instability. Destabilizing interactions lead to film rupture if these are sufficiently strong to overpower the stabilizing effect of the surface tension. The competition of forces selects a wavelength which is amplified most. The amplitude of this mode dominates the system, until eventually its maxima touch the upper electrode to form an array of liquid patterns. The EHD instability in the limit of thin films is also exploited as a lithographic technique. EHD lithography provides a novel way to induce and control the structure formation process in thin films under the influence of strong electric fields. Structures in the form of hexagonally arranged pillars are typi- 
cally formed in the presence of a homogeneous electric field. More complex shapes of patterns can be obtained by using a topographically structure top electrode. Applying a laterally varying electric field guides the instability in the direction of the highest electric field. As a consequence, the emerging structure in the film is focused towards the electrode structure which protrudes downward towards the polymer film. This results in the replication of an upper master electrode. Since the electrostatic forces are strongest for smallest electrode spacings, the time for the instability to form is much shorter. Using this lithographic approach, the extension to lateral length scales to less than $100 \mathrm{~nm}$ and aspect ratios greater than unity seems feasible.

In the first two chapters of the thesis the main aspects related to the scope of this work, the experimental approach and the relevant theoretical background for stability and hydrodynamics of thin liquid films under the influence of an electric field are introduced and discussed. Each of the following chapters are devoted to a different material or set of materials patterned by the EHD method, followed by the discussion of the unique outcomes, functionalities and the extent of the control accomplished, and an outlook towards authentication of potential applications.

In Chapter 4 a limiting factor of the slow replication dynamics of the EHD method is discussed and the theoretical framework of electrohydrodynamic instabilities leading to increased pattern formation rate is presented. Consequently, an EHL system that combines the requirements of glass-forming materials with a low viscosity in the accessible temperature window and the arrest of pattern formation during this narrow, well defined time-window is introduced for the rapid patterning. By using several lowviscosity glass forming polymers, the patterning time of EHL is significantly reduced to a few seconds compared to earlier approaches, without compromising the high fidelity of the replicated structures. The rapid pace of the EHD instability demonstrated in these experiment requires however, an improved monitoring method of the patterning process. Since current is passed through the leaky dielectric once it spans the capacitor gap, the overall capacitor current is a good measure for EHL completion. Monitoring of small but finite current that flows through the device during pattern formation is therefore exploited in this study as an in-situ control of the EHL process during this short time span. The current trace exhibits a sigmoidal shape defining the characteristic onset and completion time of the EHD process. Importantly, this study shows that, despite the much shorter patterning times, the EHD process is similar to that of high viscosity polymers and is therefore well described by a linear stability analysis. The results of this study have a technological appeal, not only because of the speed-up of the lithographic process, but also by providing an extension to additional materials that can be patterned by EHD, demonstrating the versatility of this method.

Chapter 5 presents a route towards controlled and reproducible alignment of CNTs 
during an EHD patterning process. EHD patterning of carbon nanotube-polymer composite films yields well-defined patterns on the micrometer scale along with the alignment of carbon nanotubes (CNTs) within these patterns. Composite film conductivity as a function of nanotubes content shows a substantial increase in conductivity. Conductive pathways in percolating nanotube networks are also controlled by EHD patterning, shifting the percolation threshold by $\approx 1 \%$ for the field strength used in these experiments. The preferential alignment of the nanotubes along the pillar axis during pattern formation is shown to arise from a combination of the electrostatic torque exerted on the nanotubes by the electric field and hydrodynamic shear in the elongational flow. The possibility of simultaneous structuring the nanocompsite and controlling the nanotube alignment by EHD instabilities enables an intriguing way to control the properties of the composite. The aligned CNTs are further exposed by selective polymer etching to form a CNT brush. These hybrid vertically oriented structures have potential applications for the development of flexible microelectronics, displays and biocatalytic assemblies, such as flexible field emitters, field emission devices (FEDs) or sensors.

The destabilization of multilayers by EHD patterning yields an intriguing range of readily fine-tuned hierarchical structures, each of which can be used for SERS applications. In Chapter 6, hierarchical electrohydrodynamic patterning is shown to enable the formation of 3D structures with three independent, characteristic lateral dimensions. This approach harnesses a sequential instability in multilayer thin films induced by an electric field to guide the layer material into design structures, allowing different materials to be patterned in a one-step procedure. It also offers a great variability of the structural features by the independent alteration of several parameters. Hierarchical polymer structures covered by a plasmon-active-metal (i.e., gold) effectively enhance the electromagnetic field, in the fine multiscale architecture. Individual HEHD patterned pillars function as an isolated detection centres, rendering these patterned substrates as platforms for multiplex SERS of different chemical species. For an optimized aspect ratio and geometry they give a 100-fold enhancement in SERS signals. Secondary and ternary EHD instabilities yield patterns widths that are considerably smaller (by about a factor of ten) than the primary structure size, and the different HEHD patterns show structure dependent SERS. The straightforward and robust HEHD fabrication of sub-micrometer architectures of this study are an elegant route for high throughput biological and chemical sensing.

The versatility of EHD lithography is demonstrated in Chapter 7 for the structure formaton of organic crystalline materials which at the same time promotes crystalline chain alignment in the generated patterns. Understanding the mechanism leading to orientation during the EHD-induced crystallization is essential for potential applications and the possible effects inducing vertical $c$-axis alignment of the crystal lattice 
in EHD patterned films. The application of EHL pattern formation using crystalline materials and in particular, inducing an anisotropic structure in polymer crystals is attractive, because, it extends this technique towards functionalities that can not be achieved by using amorphous or other polycrystalline materials. Orientation of crystals within the structures may give rise to interesting mechanical, electronic, optical and ferroeelctric properties, making EHL generated patterns appealing for various applications.

In Chapter 8 EHD lithography with conductive polymers is explored, aiming at patterning micro-and nano-structures with high fidelity. A spin coatable polypyrrole polymer was synthesized and films were patterned yielding well-defined conductive structures with feature sizes ranging from tens of micrometers to hundreds nanometres. While the interaction of the electric field with a dielectric matter is well understood, a somewhat different mechanism is responsible for the pattern generation in the case of conductive materials. Since, the total potential difference generated by the dipole layers at the interface is suppressed across the liquid layer, the driving force of the pattern formation in the case of a leaky dielectric polymer subjected to the EHL patterning lies in the electric field in the air gap. The patterning of PPy provides a proof-ofconcept showing that the versatile EHL patterning method can be applied to variety of conducive polymers. Submicrometer structured conducting polymers possess improved properties and performance compared with the bulk material devices and therefore, exhibit potential implementation into a variety of functional devices, including field effect transistors, organic light emitting, integrated circuits, sensors and electrochromic devices.

The combined bottom-up and top-down approach based on electrohydrodynamic formation of surface patterns in thin films and self-assembly of functional electroconductive block copolymer within these patterns is given in Chapter 9. Upon temperature or solvent-vapor annealing, initially cylindrical block copolymer microdomains oriented parallel to the substrate are destabilized by electric fields yielding the formation of microstructures perpendicular to the substrate. Within each structure the cylinders are aligned parallel with respect to the microstructure axis and to the electric field lines. These micrometer-sized patterns have an additional sub-nanostructure arising from the microphase-separated BCP morphology aligned parallel to the electric vector. Oriented nanodomains constitute an additional degree of order on the sub-10 nm scale consisting of the block copolymer crystalline stacks with the $\pi-\pi$ interactions oriented perpendicular to the electric field direction. The formation of the patterns on the three different length scales is explained in terms of the interplay of molecular self-assembly and the structural control exerted by the electrostatic forces. The presence of a vertically aligned nanodomain morphology in this system provides an 
environment with modulated interfaces between the two blocks of the liquid crystalline polymer, in which an amorphous block is assembled into a random coil directing the orientation of crystalline stacks into a well-defined order. Inside the superstructured films, ordered arrays of nanocrystals of the constituting block are aligned in smectic phase giving rise to linear dichroism. The controlled alignment of the nanodomians is also shown to improve the charge conduction toward the device electrodes. The charge transport across the entire device renders these aligned nanomorphologies appealing for a range of technological applications, in particular for organic photovoltaic cells. Moreover, the simultaneous patterning of functional block copolymer films on three length scales may open a path towards fabrication of functionalities devices for electronic, chemical and biological applications.

Finally, the advantages of present patterning technique is noteworthy. The methods are bench-top experiments, which require essentially only a hot-plate or oven and a low-voltage power supply. While the geometry of the double-plate set-up has to be well defined, this can be easily achieved by standard spinÚcoating techniques and the introduction of spacers that can be manufactured using optical lithography. Therefore the inclusion of our lithographic techniques into the established semiconductor manufacture process (i.e. into a mask aligner) should be feasible. The facile EHD patterning technique provides the ability for a straightforward design, manufacture and control of highly reliable and robust submicron patterns at low cost. Its versatility and the applications appeal are emphasized by the possibility of patterning a wide range of materials, single and multilayer film assemblies yielding varieties of hierarchical structures and anisotropic substructures. Therefore the inclusion of the studied lithographic routes into the established semiconductor manufacture process can be envisioned.

In conclusion, this thesis has presented a study on the electrohydrodynamic lithography as a versatile patterning tool on the sub-micrometre and nanometre length scales for functional materials in thin films. Founded on a simple concept with a rather generic experimental principle, this method has been successfully expanded to become a pathway for further possibilities and applications. While this study demonstrates the physics of film instabilities and their applications, the limits of this approach are not yet reached. 


\section{Bibliography}

[1] http://cloud.graphicleftovers.com/24766/501945/rows-of-chairs.jpg.

[2] http://en.wikipedia.org/.

[3] http://www.doitpoms.ac.uk/tlplib/polymers/spherulites.php.

[4] http://www.nanoshel.com/nanotechnology.php.

[5] http://www.renishawdiagnostics.com/en/klarite-sers-detection-substrates12515.

[6] Growth and morphology of cadmium chalcogenides: the synthesis of nanorods, tetrapods, and spheres from cdo and cd(o2cch3)2. J. Mater. Chem., 13(7):17051709, 2003.

[7] P.M. Ajayan, O. Stephan, C. Colliex, and D. Trauth. Aligned carbon nanotube arrays formed by cutting a polymer resin-nanotube composite. Science, 265(5176):1212-1214, 1994. cited By (since 1996) 608.

[8] and, Mansky P, Chaikin P, and Thomas EL. Monolayer films of diblock copolymer microdomains for nanolithographic applications. Journal of Materials Science, 30(8):1987-1992, 1995.

[9] Joonwon Bae, Elizabeth Glogowski, Suresh Gupta, Wei Chen, Todd Emrick, and Thomas P. Russell. Effect of nanoparticles on the electrohydrodynamic instabilities of polymer/nanoparticle thin films. Macromolecules, 41(7):2722-2726, 2008.

[10] William L. Barnes, Alain Dereux, and Thomas W. Ebbesen. Surface plasmon subwavelength optics. Nature, 424(6950):824-830, August 2003.

[11] Frank S. Bates and Glenn H. Fredrickson. Block copolymer thermodynamics: Theory and experiment. Annual Review of Physical Chemistry, 41(1):525-557, 1990.

[12] Ray H. Baughman, Anvar A. Zakhidov, and Walt A. de Heer. Carbon Nanotubesthe Route Toward Applications. Science, 297(5582):787-792, 2002.

[13] J. Bischof, D. Scherer, S. Herminghaus, and P. Leiderer. Dewetting modes of thin metallic films: Nucleation of holes and spinodal dewetting. Physical Review Letters, 77(8):1536-1539, 1996.

[14] H. Bittiger, R. H. Marchessault, and W. D. Niegisch. Crystal structure of polyє-caprolactone. Acta Crystallographica Section B, 26(12):1923-1927, Dec 1970. 
[15] C. T. Black, R. Ruiz, G. Breyta, J. Y. Cheng, M. E. Colburn, K. W. Guarini, H.C. Kim, and Y. Zhang. Polymer self assembly in semiconductor microelectronics, 2007.

[16] Eberhard Bodenschatz, Werner Pesch, and Guenter Ahlers. Recent developments in rayleigh-benard convection. Annual Review of Fluid Mechanics, 32(1):709-778, 2000.

[17] Jeffrey Bodycomb, Yoshinori Funaki, Kohtaro Kimishima, and Takeji Hashimoto. Single-grain lamellar microdomain from a diblock copolymer. Macromolecules, 32(6):2075-2077, 1999.

[18] Farhad A. Boroumand, Paul W. Fry, and David G. Lidzey. Nanoscale conjugatedpolymer light-emitting diodes. Nano Letters, 5(1):67-71, 2005.

[19] J. B. Brzoska, I. Ben Azouz, and F. Rondelez. Silanization of solid substrates: A step toward reproducibility. Langmuir, 10(11):4367-4373, 1994.

[20] Vanessa Z.-H. Chan, James Hoffman, Victor Y. Lee, Hermis Iatrou, Apostolos Avgeropoulos, Nikos Hadjichristidis, Robert D. Miller, and Edwin L. Thomas. Ordered bicontinuous nanoporous and nanorelief ceramic films from self assembling polymer precursors. Science, 286(5445):1716-1719, 1999.

[21] T.-E. Chang, A. Kisliuk, S.M. Rhodes, W.J. Brittain, and A.P. Sokolov. Conductivity and mechanical properties of well-dispersed single-wall carbon nanotube/polystyrene composite. Polymer, 47(22):7740 - 7746, 2006.

[22] J.P Chen, D Markiewicz, V.Y Lee, G Klaerner, R.D Miller, and J.C Scott. Improved efficiencies of light-emitting diodes through incorporation of charge transporting components in tri-block polymers. Synthetic Metals, 107(3):203 - 207, 1999.

[23] S. Y. Chou, P. R. Krauss, and P. J. Renstrom. Imprint lithography with 25nanometer resolution. Science, 272(5258):85-87, 1996.

[24] Brian H. Cumpston, Sundaravel P. Ananthavel, Stephen Barlow, Daniel L. Dyer, Jeffrey E. Ehrlich, Lael L. Erskine, Ahmed A. Heikal, Stephen M. Kuebler, I.-Y. Sandy Lee, Dianne McCord-Maughon, Jinqui Qin, Harald Rockel, Mariacristina Rumi, Xiang-Li Wu, Seth R. Marder, and Joseph W. Perry. Two-photon polymerization initiators for three-dimensional optical data storage and microfabrication. Nature, 398(6722):51-54, March 1999. 
[25] Ke Dai, Tianyou Peng, Dingning Ke, and Bingqing Wei. Refereeslibrariansphotocatalytic hydrogen generation using a nanocomposite of multi-walled carbon nanotubes and tio2 nanoparticles under visible light irradiation. Nanotechnology, 20:125603, 2009.

[26] M. D. Dickey, E. Collister, A. Raines, P. Tsiartas, T. Holcombe, S. V. Sreenivasan, R. T. Bonnecaze, and C. G. Willson. Photocurable pillar arrays formed via electrohydrodynamic instabilities. Chemistry of Materials, 18(8):2043-2049, 2006.

[27] W. Ding, A. Eitan, F.T. Fisher, X. Chen, D.A. Dikin, R. Andrews, L.C. Brinson, L.S. Schadler, and R.S. Ruoff. Direct observation of polymer sheathing in carbon nanotube-polycarbonate composites. Nano Letters, 3(11):1593-1597, 2003.

[28] B. Dong, D.Y. Zhong, L.F. Chi, and H. Fuchs. Patterning of conducting polymers based on a random copolymer strategy: Toward the facile fabrication of nanosensors exclusively based on polymers. Advanced Materials, 17(22):2736-2741, 2005.

[29] C. J. Drury, C. M. J. Mutsaers, C. M. Hart, M. Matters, and D. M. de Leeuw. Low-cost all-polymer integrated circuits, 1998.

[30] T.W. Ebbesen, P.M. Ajayan, H. Hiura, and K. Tanigaki. Purification of nanotubes. Nature, 367:519, 1994.

[31] Florian Ebert and Thomas Thurn-Albrecht. Controlling the orientation of semicrystalline polymers by crystallization in magnetic fields. Macromolecules, 36(23):8685-8694, 2003.

[32] Dominik Eder. Chem. Rev., 110(3):1348-1385, 2010.

[33] M Endo, Y.A Kim, T Hayashi, K Nishimura, T Matusita, K Miyashita, and M.S Dresselhaus. Vapor-grown carbon fibers (vgcfs): Basic properties and their battery applications. Carbon, 39(9):1287 - 1297, 2001.

[34] Yonngin S. Kyunggi D. Eung, J. and J.S. Kwan. Method for making polypyrrole. Patent WO 02/10251, 2002.

[35] Shoushan Fan, Michael G. Chapline, Nathan R. Franklin, Thomas W. Tombler, Alan M. Cassell, and Hongjie Dai. Self-Oriented Regular Arrays of Carbon Nanotubes and Their Field Emission Properties. Science, 283(5401):512-514, 1999.

[36] Michael J Fasolka and Anne M Mayes. Block copolymer thin films: Physics and applications1. Annual Review of Materials Research, 31(1):323-355, 2001. 
[37] M. Fleischmann, P.J. Hendra, and A.J. McQuillan. Raman spectra of pyridine adsorbed at a silver electrode. Chemical Physics Letters, 26(2):163 - 166, 1974.

[38] G. Floudas, L. Hilliou, D. Lellinger, and I. Alig. Shear-induced crystallization of poly(e-caprolactone). 2. evolution of birefringence and dichroism. Macromolecules, 33(17):6466-6472, 2000.

[39] SP Fodor, JL Read, MC Pirrung, L Stryer, AT Lu, and D Solas. Light-directed, spatially addressable parallel chemical synthesis. Science, 251(4995):767-773, 1991.

[40] D. Frenkel, H. N. W. Lekkerkerker, and A. Stroobants. Thermodynamic stability of a smectic phase in a system of hard rods. Nature, 332(6167):822-823, April 1988.

[41] J. Frenkel. On tonks theory of liquid surface rupture by a uniform electric filed. Phys. Z. Sowjetunion, 8:675-679, 1935.

[42] P. Goldberg-Oppenheimer and U Steiner. Rapid electrohydrodynamic lithography using low viscosity resists. Small, 6:1248-1254, 2010.

[43] Pola Goldberg-Oppenheimer, Dominik Eder, and Ullrich Steiner. Carbon nanotube alignment via electrohydrodynamic patterning of nanocomposites. Advanced Functional Materials, 21(10):1895-1901, 2011.

[44] Pola Goldberg-Oppenheimer and Oren Regev. Exploring a nanotube dispersion mechanism with gold-labeled proteins via cryo-tem imaging. Small, 3(11):18941899, NOV 2007.

[45] Xiaoyi Gong, Jun Liu, Suresh Baskaran, Roger D. Voise, and James S. Young. Surfactant-assisted processing of carbon nanotube/polymer composites. Chemistry of Materials, 12(4):1049-1052, April 2000.

[46] T. Granlund, T. Nyberg, L. Stolz Roman, M. Svensson, and O. Inganaes. Patterning of polymer light-emitting diodes with soft lithography. Advanced Materials, $12(4): 269-273,2000$.

[47] I.W. Hamley, E.L. Hiscutt, Y.-W. Yang, and C. Booth. Dewetting of thin block copolymer films. Journal of Colloid and Interface Science, 209(1):255 - 260, 1999.

[48] P. Harder, M. Grunze, R. Dahint, G. M. Whitesides, and P. E. Laibinis. Molecular conformation in oligo(ethylene glycol)-terminated self-assembled monolayers on gold and silver surfaces determines their ability to resist protein adsorption. The Journal of Physical Chemistry B, 102(2):426-436, 1998. 
[49] S Harkema. Capillary instabilities in thin polymer films. PhD Thesis, http://irs.ub.rug.nl/ppn/291147801, 2006.

[50] S. Harkema and U. Steiner. Hierarchical pattern formation in thin polymer films using an electric field and vapor sorption. Advanced Functional Materials, 15(12):2016-2020, 2005.

[51] T. Hayakawa and S. Horiuchi. From angstroms to micrometers: self-organized hierarchical structure within a polymer film. Angewandte Chemie, 42:2285, 2003.

[52] J. Heier, J. Groenewold, and U. Steiner. Pattern formation in thin polymer films by spatially modulated electric fields. Soft Matter, 5:3997-4005, 2009.

[53] A. M. Higgins and R. A. L. Jones. Anisotropic spinodal dewetting as a route to self-assembly of patterned surfaces. Nature, 404(6777):476-478, 2000.

[54] Hidefumi Hiura, Thomas W. Ebbesen, and Katsumi Tanigaki. Opening and purification of carbon nanotubes in high yields. Adv. Mater., 7(3):275-276, 1995.

[55] S.A. Hoenig. Cleaning surfaces with dry ice. Compressed Air Magazine, 91 (8):22-24, 1986.

[56] Scott J. Hollister. Porous scaffold design for tissue engineering. Nat Mater, 4(7):518-524, July 2005.

[57] H. Hou, J.J. Ge, J. Zeng, Q. Li, D.H. Reneker, A. Greiner, and S.Z.D. Cheng. Electrospun polyacrylonitrile nanofibers containing a high concentration of wellaligned multiwall carbon nanotubes. Chemistry of Materials, 17(5):967-973, 2005.

[58] R. L. Howey. Mantids, stick insects and centipedes. Micscape Magazine, 2007.

[59] Lu Hu and Gang Chen. Analysis of optical absorption in silicon nanowire arrays for photovoltaic applications. Nano Letters, 7(11):3249-3252, November 2007.

[60] Sven Huttner, Michael Sommer, and Mukundan Thelakkat. n-type organic field effect transistors from perylene bisimide block copolymers and homopolymers. Applied Physics Letters, 92(9):093302, 2008.

[61] Z. Jin, K.P. Pramoda, S.H. Goh, and G. Xu. Poly(vinylidene fluoride)-assisted melt-blending of multi-walled carbon nanotube/poly(methyl methacrylate) composites. Materials Research Bulletin, 37(2):271-278, 2002. 
[62] Jaebum Joo, Brian Y. Chow, and Joseph M. Jacobson. Nanoscale patterning on insulating substrates by critical energy electron beam lithography. Nano Letters, 6(9):2021-2025, 2006.

[63] M. Kahl, E. Voges, S. Kostrewa, C. Viets, and W. Hill. Periodically structured metallic substrates for sers. Sensors and Actuators B: Chemical, 51(1-3):285$291,1998$.

[64] R. S. Kane, R. E. Cohen, and R. Silbey. Synthesis of pbs nanoclusters within block copolymer nanoreactors. Chemistry of Materials, 8(8):1919-1924, 1996.

[65] Eugenii Katz, Itamar Willner, and Joseph Wang. Electroanalytical and bioelectroanalytical systems based on metal and semiconductor nanoparticles. Electroanalysis, 16(1-2):19-44, 2004.

[66] Howard. E. Katz. Chemically sensitive field-effect transistors and chemiresistors: New materials and device structures. Electroanalysis, 16(22):1837-1842, 2004.

[67] G. Kickelbick. Introduction to Hybrid Materials, in Hybrid Materials: Synthesis, Characterization, and Applications. Wiley-VCH Verlag GmbH \& Co. KGaA, Weinheim, 2007.

[68] G. Kim and M. Libera. Morphological development in solvent-cast polystyreneco-polybutadiene-co-polystyrene (sbs) triblock copolymer thin films. Macromolecules, 31(8):2569-2577, 1998.

[69] S.H. Kim, M.J. Misner, T. Xu, M. Kimura, and T.P. Russell. Highly oriented and ordered arrays from block copolymers via solvent evaporation. Advanced Materials, 16(3):226-231, 2004.

[70] T. Kimura, H. Ago, M. Tobita, S. Ohshima, M. Kyotani, and M. Yumura. Polymer composites of carbon nanotubes aligned by a magnetic field. Advanced $M a-$ terials, 14(19):1380-1383, 2002. cited By (since 1996) 122.

[71] Tsunehisa Kimura, Masafumi Yamato, Wataru Koshimizu, Minako Koike, and Takahiko Kawai. Magnetic orientation of polymer fibers in suspension. Langmuir, $16(2): 858-861,2000$.

[72] Katrin Kneipp, Yang Wang, Harald Kneipp, Lev T. Perelman, Irving Itzkan, Ramachandra R. Dasari, and Michael S. Feld. Single molecule detection using surface-enhanced raman scattering (sers). Phys. Rev. Lett., 78(9):1667-1670, Mar 1997. 
[73] Satish Kumar, Thuy D. Dang, Fred E. Arnold, Arup R. Bhattacharyya, Byung G. Min, Xiefei Zhang, Richard A. Vaia, Cheol Park, W. Wade Adams, Robert H. Hauge, Richard E. Smalley, Sivarajan Ramesh, and Peter A. Willis. Synthesis, structure, and properties of pbo/swnt composites. Macromolecules, 35(24):90399043, November 2002.

[74] E. Kymakis, I. Alexandrou, and G. A. J. Amaratunga. High open-circuit voltage photovoltaic devices from carbon-nanotube-polymer composites. Journal of Applied Physics, 93(3):1764-1768, 2003.

[75] E. Kymakis and G. A. J. Amaratunga. Single-wall carbon nanotube/conjugated polymer photovoltaic devices. Applied Physics Letters, 80(1):112-114, 2002.

[76] R. G. H. Lammertink, M. A. Hempenius, J. E. van den Enk, V. Z.H. Chan, E. L. Thomas, and G. J. Vancso. Nanostructured thin films of organic and organometallic block copolymers: One-step lithography with poly(ferrocenylsilanes) by reactive ion etching. Advanced Materials, 12(2):98103,2000 .

[77] L.D Landau and E.M. Lifshitz. Fluid mechanics. London: Pergamon Press, 1959.

[78] KA Leach, ZQ Lin, and TP Russell. Early stages in the growth of electric fieldinduced surface fluctuations. Macromolecules, 38(11):4868-4873, Jan 2005.

[79] Heon Sang Lee, Chang Hun Yun, Heon Mo Kim, and Cheol Jin Lee. Persistence length of multiwalled carbon nanotubes with static bending. The Journal of Physical Chemistry C, 111(51):18882-18887, 2007.

[80] M.C. LeMieux, M. Roberts, S. Barman, Y.W. Jin, J.M. Kim, and Z. Bao. Selfsorted, aligned nanotube networks for thin-film transistors. Science, 321:101-104, 2008.

[81] Cheng Li, Yuhong Chen, Yubing Wang, Zafar Iqbal, Manish Chhowalla, and Somenath Mitra. A fullerene-single wall carbon nanotube complex for polymer bulk heterojunction photovoltaic cells. J. Mater. Chem., 17(23):2406-2411, 2007.

[82] Minqin Li, Shengqing Xu, and Eugenia Kumacheva. Convection in polymeric fluids subjected to vertical temperature gradients. Macromolecules, 33(13):49724978, 2000.

[83] X.Z Li. Jecp/pced simulation of polycrystalline eelctron diffraction pattern and phase identification. Ultramicroscopy, 99:257-261, 2004. 
[84] X.Z. Li. Quantitative analysis of polycrystalline electron diffraction patterns. Microscopy and Microanalysis, 13:1-2, 2007.

[85] Z. Q. Lin, T. Kerle, S. M. Baker, D. A. Hoagland, E. Schaffer, U. Steiner, and T. P. Russell. Electric field induced instabilities at liquid/liquid interfaces. Journal of Chemical Physics, 114(5):2377-2381, 2001.

[86] Z. Q. Lin, T. Kerle, T. P. Russell, E. Schaffer, and U. Steiner. Structure formation at the interface of liquid liquid bilayer in electric field. Macromolecules, 35(10):3971-3976, 2002.

[87] Huttner-S Chiche A Thelakkat M. Lindner, S.M. and G. Krausch. Charge separation at self-assembled nanostructured bulk interface in block copolymers. Angewandte Chemie, 45:3364-3368, 2006.

[88] Jimmy Lowe and Steven Holdcroft. Synthesis and photolithography of polymers and copolymers based on poly(3-(2-(methacryloyloxy)ethyl)thiophene). Macromolecules, 28(13):4608-4616, 1995.

[89] A. Schuster M. Schadt, H. Seiberle. Optical patterning of multi-domain liquidcrystal displays with wide viewing angles. Nature, 381:212, 1996.

[90] B. McCarthy, J. N. Coleman, R. Czerw, A. B. Dalton, M. in het Panhuis, A. Maiti, A. Drury, P. Bernier, J. B. Nagy, B. Lahr, H. J. Byrne, D. L. Carroll, and W. J. Blau. A microscopic and spectroscopic study of interactions between carbon nanotubes and a conjugated polymer. The Journal of Physical Chemistry B, 106(9):2210-2216, 2002.

[91] J. R. Melcher. Electrohydrodynamic and magnetohydrodynamic surface waves and instabilities. Physics of Fluids, 4:1348-1354, 1961.

[92] J. R. Melcher. Field-Coupled Surface Waves. MIT Press, Cambridge, Mass., 1963.

[93] J. Melngailis, A. A. Mondelli, Ivan L. Berry III, and R. Mohondro. A review of ion projection lithography. Journal of Vacuum Science \& Technology B: Microelectronics and Nanometer Structures, 16(3):927-957, 1998.

[94] E. Menard, M. A. Meitl, Y. G. Sun, J. U. Park, D. J. L. Shir, Y. S. Nam, S. Jeon, and J. A. Rogers. Micro- and nanopatterning techniques for organic electronic and optoelectronic systems. Chemical Reviews, 107(4):1117-1160, 2007. 
[95] E.T. Mickelson, C.B. Huffman, A.G. Rinzler, R.E. Smalley, R.H. Hauge, and J.L. Margrave. Fluorination of single-wall carbon nanotubes. Chemical Physics Letters, 296(1-2):188-194, 1998.

[96] M. D. Morariu, N. E. Voicu, E. Schaffer, Z. Q. Lin, T. P. Russell, and U. Steiner. Hierarchical structure formation and pattern replication induced by an electric field. Nature Materials, 2(1):48-52, 2003.

[97] Lu M. Urbas A.M. Ehrichs E.E. Jeaeger H.M. Mansky P. Morkved, T.L. and T.P. Russell. Local control of microdomain orientation in diblock copolymer thin films with electric fields. Science, 273 (5277):931-933, 1996.

[98] Jun-ichi Nakamura, Yutaka Sasabe, Tetsuya Takahashi, and Michio Inagaki. Lithium secondary battery using vapor grown carbon fibers as a negative electrode and analysis of the electrode mechanism by tem observation. The Transactions of the Institute of Electrical Engineers of Japan. A, 115(4):349-356, 1995.

[99] Einat Nativ-Roth, Rina Shvartzman-Cohen, Celine Bounioux, Marc Florent, Dongsheng Zhang, Igal Szleifer, and Rachel Yerushalmi-Rozen. Physical adsorption of block copolymers to swnt and mwnt: A nonwrapping mechanism. Macromolecules, 40(10):3676-3685, May 2007.

[100] Shuming Nie and Steven R. Emory. Probing single molecules and single nanoparticles by surface-enhanced raman scattering. Science, 275(5303):1102-1106, 1997.

[101] Alexander Oron, Stephen H. Davis, and S. George Bankoff. Long-scale evolution of thin liquid films. Rev. Mod. Phys., 69(3):931-980, Jul 1997.

[102] Ekmel Ozbay. Plasmonics: Merging photonics and electronics at nanoscale dimensions. Science, 311(5758):189-193, 2006.

[103] R. Parashkov, E. Becker, T. Riedl, H.-H. Johannes, and W. Kowalsky. Microcontact printing as a versatile tool for patterning organic field-effect transistors. Advanced Materials, 17(12):1523-1527, 2005.

[104] Cheol Park, Zoubeida Ounaies, Kent A. Watson, Roy E. Crooks, Joseph Smith, Sharon E. Lowther, John W. Connell, Emilie J. Siochi, Joycelyn S. Harrison, and Terry L. St. Clair. Dispersion of single wall carbon nanotubes by in situ polymerization under sonication. Chemical Physics Letters, 364(3-4):303 - 308, 2002. 
[105] Miri Park, Christopher Harrison, Paul M. Chaikin, Richard A. Register, and Douglas H. Adamson. Block copolymer lithography: Periodic arrays of 1011 holes in 1 square centimeter. Science, 276(5317):1401-1404, 1997.

[106] L. F. Pease and W. B. Russel. Linear stability analysis of thin leaky dielectric films subjected to electric fields. Journal of Non-Newtonian Fluid Mechanics, 102(2):233-250, 2002. Sp. Iss. SI.

[107] L. F. Pease and W. B. Russel. Electrostatically induced submicron patterning of thin perfect and leaky dielectric films: A generalized linear stability analysis. Journal of Chemical Physics, 118(8):3790-3803, 2003.

[108] L. F. Pease and W. B. Russel. Limitations on length scales for electrostatically induced submicrometer pillars and holes. Langmuir, 20(3):795-804, 2004.

[109] L. F. Pease and W. B. Russel. Charge driven, electrohydrodynamic patterning of thin films. Journal of Chemical Physics, 125(18), 2006.

[110] Nicolas M.B. Perney, Jeremy J. Baumberg, Majd E. Zoorob, Martin D. B. Charlton, Sven Mahnkopf, and Caterina M. Netti. Tuning localized plasmons in nanostructured substrates for surface-enhanced raman scattering. Opt. Express, 14(2):847-857, Jan 2006.

[111] B. Pradhan, R.R. Kohlmeyer, and J. Chen. Fabrication of in-plane aligned carbon nanotube-polymer composite thin films. Carbon, 48(1):217-222, 2010.

[112] D. Qian, E. C. Dickey, R. Andrews, and T. Rantell. Load transfer and deformation mechanisms in carbon nanotube-polystyrene composites. Applied Physics Letters, 76(20):2868-2870, 2000.

[113] D. Qin, Y. N. Xia, J. A. Rogers, R. J. Jackman, X. M. Zhao, and G. M. Whitesides. Microfabrication, microstructures and microsystems. In Microsystem Technology in Chemistry and Life Science, volume 194 of Topics in Current Chemistry, pages 1-20. 1998.

[114] CVKKS. Raman. A new type of secondary radiation. Nature, 121:501, 1928.

[115] Kumaran Ramanathan, Mangesh A. Bangar, Minhee Yun, Wilfred Chen, Ashok Mulchandani, and Nosang V. Myung. Individually addressable conducting polymer nanowires array. Nano Letters, 4(7):1237-1239, 2004.

[116] G. Reiter, A. Sharma, A. Casoli, M. O. David, R. Khanna, and P. Auroy. Destabilising effect of long-range forces in thin liquid films on wettable substrates. Europhysics Letters, 46(4):512-518, 1999. 
[117] M. Reynolds. Stability of electrostatically supported fluid column. Physics of Fluids, 8:161-170, 1965.

[118] Somani RH, Yang L, Zhu L, and Hsiao BS. Flow-induced shish-kebab precursor structures in entangled polymer melts. Polymer, 46(20):8587-8623, 2005.

[119] A. Rinaldi. Naturally better. science and technology are looking to nature's successful designs for inspiration. EMBO reports, 8:995-999, 2007.

[120] Michael W. Rowell, Mark A. Topinka, Michael D. McGehee, Hans-Jurgen Prall, Gilles Dennler, Niyazi Serdar Sariciftci, Liangbing Hu, and George Gruner. Organic solar cells with carbon nanotube network electrodes. Applied Physics Letters, 88(23):233506, 2006.

[121] Ruckenst.E and R. K. Jain. Spontaneous rupture of thin liquid-films. Journal of the Chemical Society-Faraday Transactions Ii, 70:132-147, 1974.

[122] J.E. Ruedy. Design and performance of an electron diffraction. Rev. Scientific Instruments, 11:292-295, 1940.

[123] B. Safadi, R. Andrews, and E. A. Grulke. Multiwalled carbon nanotube polymer composites: Synthesis and characterization of thin films. Journal of Applied Polymer Science, 84(14):2660-2669, 2002.

[124] J Sandler, M.S.P Shaffer, T Prasse, W Bauhofer, K Schulte, and A.H Windle. Development of a dispersion process for carbon nanotubes in an epoxy matrix and the resulting electrical properties. Polymer, 40(21):5967 - 5971, 1999.

[125] J. K. W. Sandler, J. E. Kirk, I. A. Kinloch, M. S. P. Shaffer, and A. H. Windle. Ultra-low electrical percolation threshold in carbon-nanotube-epoxy composites. Polymer, 44(19):5893 - 5899, 2003. In Honour of Ian Ward's 75th Birthday.

[126] Grubb David T. Meyers Gregory F. Sawyer, Linda. Polymer Microscopy. Springer, 2008.

[127] E Schaffer. Instabilities in thin polymer films: structure formation and pattern transfer. PhD Thesis, http://www.ub.unikonstanz.de/kops/volltexte/2002/779/, 2001.

[128] E. Schaffer, S. Harkema, M. Roerdink, R. Blossey, and U. Steiner. Morphological instability of a confined polymer film in a thermal gradient. Macromolecules, 36(5):1645-1655, 2003. 
[129] E. Schaffer, S. Harkema, M. Roerdink, R. Blossey, and U. Steiner. Thermomechanical lithography: Pattern replication using a temperature gradient driven instability. Advanced Materials, 15(6):514-517, 2003.

[130] E. Schaffer, T. Thurn-Albrecht, T. P. Russell, and U. Steiner. Electrically induced structure formation and pattern transfer. Nature, 403(6772):874-877, 2000.

[131] E. Schaffer, T. Thurn-Albrecht, T. P. Russell, and U. Steiner. Electrohydrodynamic instabilities in polymer films. Europhysics Letters, 53(4):518-524, 2001.

[132] Kirk S. Schanze, Troy S. Bergstedt, and Brian T. Hauser. Photolithographic patterning of electroactive polymer films and electrochemically modulated optical diffraction gratings. Advanced Materials, 8(6):531-534, 1996.

[133] B Schrader and D Bougeard. Infrared and raman spectroscopy: methods and applications. Wiley-VCH, Weinheim, 1995.

[134] D. Schutze, B. Knabe, M. Ackermann, and K. Buse. Orientation of colloidally suspended linbo[3] nanocrystals in externally applied electric fields. Applied Physics Letters, 97(24):242908, 2010.

[135] R. Seemann, S. Herminghaus, and K. Jacobs. Dewetting patterns and molecular forces: A reconciliation. Physical Review Letters, 86(24):5534-5537, 2001.

[136] R. A. Segalman, H. Yokoyama, and E. J. Kramer. Graphoepitaxy of spherical domain block copolymer films. Advanced Materials, 13(15):1152-1155, 2001.

[137] Rahul Sen, Bin Zhao, Daniel Perea, Mikhail E. Itkis, Hui Hu, James Love, Elena Bekyarova, and Robert C. Haddon. Preparation of single-walled carbon nanotube reinforced polystyrene and polyurethane nanofibers and membranes by electrospinning. Nano Letters, 4(3):459-464, March 2004.

[138] Robert Sherman, Drew Hirt, and Ronald Vane. Surface cleaning with the carbon dioxide snow jet. volume 12, pages 1876-1881. AVS, 1994.

[139] P. Silberzan, L. Leger, D. Ausserre, and J. J. Benattar. Silanation of silica surfaces. a new method of constructing pure or mixed monolayers. Langmuir, 7(8):1647-1651, 1991.

[140] Th. Birendra Singh and Niyazi Serdar Sariciftci. Progress in plastic electronics devices. Annual Review of Materials Research, 36(1):199-230, 2006. 
[141] Henning Sirringhaus, Nir Tessler, and Richard H. Friend. Integrated optoelectronic devices based on conjugated polymers. Science, 280(5370):1741-1744, 1998.

[142] Nicolas Sluzarenko, Bertrand Heurtefeu, Maryse Maugey, Cecile Zakri, Philippe Poulin, and SĖbastien Lecommandoux. Diblock copolymer stabilization of multiwall carbon nanotubes in organic solvents and their use in composites. Carbon, 44(15):3207 - 3212, 2006.

[143] Rajesh H. Somani, Benjamin S. Hsiao, Aurora Nogales, Srivatsan Srinivas, Andy H. Tsou, Igors Sics, Francisco J. Balta-Calleja, and Tiberio A. Ezquerra. Structure development during shear flow-induced crystallization of i-pp:âĂL' insitu small-angle x-ray scattering study. Macromolecules, 33(25):9385-9394, 2000.

[144] Huettner S. Sommer, M. and M. Thelakkat. Donor-acceptor block copolymers for photovoltaic applications. Journal of Materials Chemistry, 20:10788-10797, 2010.

[145] Michael Sommer, Sven Huttner, Stefanie Wunder, and Mukundan Thelakkat. Electron-conducting block copolymers: Morphological, optical, and electronic properties. Advanced Materials, 20(13):2523-2527, 2008.

[146] G. Sonmez, H.B. Sonmez, C.K.F. Shen, and F. Wudl. Red, green, and blue colors in polymeric electrochromics. Advanced Materials, 16(21):1905-1908, 2004.

[147] Joshua M. Spurgeon, Harry A. Atwater, and Nathan S. Lewis. A comparison between the behavior of nanorod array and planar cd(se, te) photoelectrodes. The Journal of Physical Chemistry C, 112(15):6186-6193, April 2008.

[148] Goring P. Prabhukaran M. Gosele U. Hempel E. Steinhart, M. and T. ThurnAlbrecht. Coherent kinetic control over crystal orientation in macroscopic ensembles of polymer nanorods and nanotubes. PRL, 97:027801, 2006.

[149] J. W. Swan. Stress and other effects produced in resin and in a viscid compound of resin and oil by electrification. Proceedings of the Royal Society, 62:38-46, 1897.

[150] D. Tabor and R.H.S. Winterton. The direct measurement of normal and retarded van der waals forces. Proceding of the Royal Society A, 312:435-450, 1969.

[151] Sander J. Tans, Alwin R. M. Verschueren, and Cees Dekker. Room-temperature transistor based on a single carbon nanotube. Nature, 393:49-52, 1998. 
[152] G.I. Taylor and A.D. Mcewan. The stability of horizontal fluid interface in a vertical electric field. The Journal of Fluid Mechanics, 22:1-15, 1965.

[153] Manuel Thary, Victor Racine, Matthieu Piel, Anne Papin, Ariane Dimitrov, Yong Chen, Jean-Baptiste Sibarita, and Michel Bornens. Anisotropy of cell adhesive microenvironment governs cell internal organization and orientation of polarity. Proceedings of the National Academy of Sciences, 103(52):19771-19776, 2006.

[154] Erik T. Thostenson, Zhifeng Ren, and Tsu-Wei Chou. Advances in the science and technology of carbon nanotubes and their composites: a review. Composites Science and Technology, 61(13):1899 - 1912, 2001.

[155] E.T. Thostenson and T.-W. Chou. Aligned multi-walled carbon nanotubereinforced composites: Processing and mechanical characterization. Journal of Physics D: Applied Physics, 35(16):L77-L80, 2002. cited By (since 1996) 205.

[156] T. Thurn-Albrecht, J. DeRouchey, T. P. Russell, and H. M. Jaeger. Overcoming interfacial interactions with electric fields. Macromolecules, 33(9):3250-3253, 2000.

[157] T. Thurn-Albrecht, J. Schotter, G. A. Kedstle, N. Emley, T. Shibauchi, L. KrusinElbaum, K. Guarini, C. T. Black, M. T. Tuominen, and T. P. Russell. Ultrahighdensity nanowire arrays grown in self-assembled diblock copolymer templates. Science, 290(5499):2126-2129, 2000.

[158] Thomas Thurn-Albrecht, Jason DeRouchey, Thomas P. Russell, and Rainer Kolb. Pathways toward electric field induced alignment of block copolymers. Macromolecules, 35(21):8106-8110, 2002.

[159] L. Tonks. A theory of liquid surface rupture by a uniform electric field. Physical Review, 48:562-568, 1935.

[160] J.J. Trillat and T.V. Hirsch. Diffrcation des 'electrones par cristaux uniques'. Jour. de Physiques et le Radium, 3:185, 1932.

[161] Sami Valkama, Harri Kosonen, Janne Ruokolainen, Tomi Haatainen, Mika Torkkeli, Ritva Serimaa, Gerrit ten Brinke, and Olli Ikkala. Self-assembled polymeric solid films with temperature-induced large and reversible photonic-bandgap switching. Nat Mater, 3(12):872-876, December 2004.

[162] R. Verma, A. Sharma, K. Kargupta, and J. Bhaumik. Electric field induced instability and pattern formation in thin liquid films. Langmuir, 21(8):3710$3721,2005$. 
[163] Marcelo A Villar, Daniel R Rueda, Fernando Ania, and Edwin L Thomas. Study of oriented block copolymers films obtained by roll-casting. Polymer, 43(19):5139 $-5145,2002$.

[164] The vintage technology association (VTA). Mysterious artifacts. MilitaryIndustrial Research \& Preservation, 2008.

[165] Ludwigs S. Crossland. E.J.W. Andrew P. Voicu, N.E. and U. Steiner. Solventvapor assisted imprint lithography. Advanced Materials, 19 (5):757-761, 2007.

[166] N. E. Voicu, S. Harkema, and U. Steiner. Electric-field-induced pattern morphologies in thin liquid films. Advanced Functional Materials, 16(7):926-934, 2006.

[167] N. E. Voicu, S. Ludwigs, and U. Steiner. Alignment of lamellar block copolymers via electrohydrodynamic-driven micropatterning. Advanced Materials, 20(16):3022-3027, 2008.

[168] N. E. Voicu, M. S. M. Saifullah, K. R. V. Subramanian, M. E. Welland, and U. Steiner. Tio2 patterning using electro-hydrodynamic lithography. Soft Matter, $3(5): 554-557,2007$.

[169] A. Vrij. Discuss. Faraday Soc., 42:23, 1966.

[170] J. Z. Wang, Z. H. Zheng, H. W. Li, W. T. S. Huck, and H. Sirringhaus. Dewetting of conducting polymer inkjet droplets on patterned surfaces. Nat Mater, 3(3):171176, March 2004.

[171] J.R. White. The use of electron diffraction intensities to detect imperfect fibre orientation in very thi films. Thin Solid Films, 22:11-21, 1974.

[172] SS Wong, E Joselevich, AT Woolley, CL Cheung, and CM Lieber. Covalently functionalized nanotubes as nanometre-sized probes in chemistry and biology. Nature, 394(6688):52-55, JUL 21998.

[173] L. Wu and S. Y. Chou. Dynamic modeling and scaling of nanostructure formation in the lithographically induced self-assembly and self-construction. Applied Physics Letters, 82(19):3200-3202, 2003.

[174] N. Wu, L. F. Pease, and W. B. Russel. Toward large-scale alignment of electrohydrodynamic patterning of thin polymer films. Advanced Functional Materials, 16(15):1992-1999, 2006. 
[175] Ning Wu and William B Russel. Micro- and nano-patterns created via electrohydrodynamic instabilities. Nano Today, 4(2):180-192, Jan 2009.

[176] F. B. Wyart, P. Martin, and C. Redon. Liquid-liquid dewetting. Langmuir, 9(12):3682-3690, 1993.

[177] Y. N. Xia and G. M. Whitesides. Soft lithography. Annual Review of Materials Science, 28:153-184, 1998.

[178] Younan Xia and Georgea. M. Whitesides. Softlithographie. Angewandte Chemie, 110(5):568-594, 1998.

[179] Hongqi Xiang, Yao Lin, and Thomas P. Russell. Electrically induced patterning in block copolymer films. Macromolecules, 37(14):5358-5363, 2004.

[180] Shengqing $\mathrm{Xu}$ and Eugenia Kumacheva. Ordered morphologies in polymeric films produced by replication of convection patterns. Journal of the American Chemical Society, 124(7):1142-1143, 2002.

[181] Bo Yan, Anupama Thubagere, W. Ranjith Premasiri, Lawrence D. Ziegler, Luca Dal Negro, and B. M. Reinhard. Engineered sers substrates with multiscale signal enhancement: Nanoparticle cluster arrays. ACS Nano, 3(5):1190-1202, 2009. PMID: 19354266.

[182] Peidong Yang, Gernot Wirnsberger, Howard C. Huang, Steven R. Cordero, Michael D. McGehee, Brian Scott, Tao Deng, George M. Whitesides, Bradley F. Chmelka, Steven K. Buratto, and Galen D. Stucky. Mirrorless lasing from mesostructured waveguides patterned by soft lithography. Science, 287(5452):465-467, 2000.

[183] Junrong Yu, Kangbo Lu, Erwan Sourty, Nadia Grossiord, Cor E. Koning, and Joachim Loos. Characterization of conductive multiwall carbon nanotube/polystyrene composites prepared by latex technology. Carbon, 45(15):2897 - 2903, 2007. 